\title{
Bone regeneration
}

Citation for published version (APA):

Bách, L. Q. (2018). Bone regeneration: Exploring a plethora of approaches. [Doctoral Thesis, Maastricht University]. Maastricht University. https://doi.org/10.26481/dis.20181218lb

Document status and date:

Published: 01/01/2018

DOI:

$10.26481 /$ dis.20181218lb

Document Version:

Publisher's PDF, also known as Version of record

\section{Please check the document version of this publication:}

- A submitted manuscript is the version of the article upon submission and before peer-review. There can be important differences between the submitted version and the official published version of record.

People interested in the research are advised to contact the author for the final version of the publication, or visit the DOI to the publisher's website.

- The final author version and the galley proof are versions of the publication after peer review.

- The final published version features the final layout of the paper including the volume, issue and page numbers.

Link to publication

\footnotetext{
General rights rights.

- You may freely distribute the URL identifying the publication in the public portal. please follow below link for the End User Agreement:

www.umlib.nl/taverne-license

Take down policy

If you believe that this document breaches copyright please contact us at:

repository@maastrichtuniversity.nl

providing details and we will investigate your claim.
}

Copyright and moral rights for the publications made accessible in the public portal are retained by the authors and/or other copyright owners and it is a condition of accessing publications that users recognise and abide by the legal requirements associated with these

- Users may download and print one copy of any publication from the public portal for the purpose of private study or research.

- You may not further distribute the material or use it for any profit-making activity or commercial gain

If the publication is distributed under the terms of Article $25 \mathrm{fa}$ of the Dutch Copyright Act, indicated by the "Taverne" license above, 


\section{BONE REGENERATION \\ EXPLORING A PLETHORA OF APPROACHES}

By

Lê Quang Bách 
Copyright ( 2018 by Le Quang Bach

All rights reserved. This book or any portion thereof may not be reproduced or used in any manner whatsoever without the express written permission of the author except for the use of brief quotations in a book review.

Layout by Le Quang Bach

Book cover by Nguyen Van Nha

Printed in The Netherlands

ISBN 9-789492-597199

The research presented in this dissertation was performed from 2010 to 2014 at the Tissue Regeneration department at the University of Twente, The Netherlands.

The research was financially supported by the Pieken in de Delta Osst Nederland (PIDON) grant 91014.

The printing of this dissertation was sponsored by the Maastricht University, The Netherlands. 


\title{
BONE REGENERATION \\ EXPLORING A PLETHORA OF APPROACHES
}

\author{
DISSERTATION
}

to obtain the degree of Doctor at the Maastricht University, on the authority of the Rector Magnificus,

Prof. Rianne Letschert

in accordance with the decision of the Board of Deans,

to be defended in public

on December $18^{\text {th }} 2018$, at 16.00 hours

by

Le Quang Bach 


\section{Supervisor:}

Prof. dr. J. de Boer

\section{Co-supervisor:}

Dr. V. LaPointe

\section{Assessment Committee:}

Prof. dr. P. Habibovic (voorzitter)

Prof. dr. R.A. Bank, University Medical Center Groningen

Prof. dr. J. Klein-Nulend, Vrije Universiteit Amsterdam

Dr. T. Welting 


\title{
Table of contents
}

\author{
1. Chapter 1 - Introduction \\ $p 1$
}

2. Chapter 2

(Review) The Components of Bone and What They Can Teach Us about Regeneration P9

3. Chapter 3

High-Throughput Screening Assay for the Identification of Compounds Enhancing Collagenous Extracellular Matrix Production by ATDC5 Cells P29

4. Chapter 4

An Approach to In Vitro Manufacturing of Hypertrophic Cartilage Matrix for

Bone Repair

P49

5. Chapter 5

Micro-Topographies Promote Late Chondrogenic Differentiation

Markers in the ATDC5 Cell Line

P73

6. Chapter 6

Generation of a BMP-2 reporter MG63 cell line using CRISPR/Cas9

mediated homologous recombination

$$
\text { P95 }
$$

7. Chapter 7 - Discussion

$$
\text { P105 }
$$

8. Valorization Addendum

$$
\text { p111 }
$$

9. Summary

p115

10. Samenvatting

$$
\text { p117 }
$$

11. Acknowledgement - And a little bit about the journey

$$
\text { p119 }
$$

12. Curriculum vitae

$$
\text { p122 }
$$





\section{Chapter 1 - Introduction}

$\mathrm{B}$ one - the skeleton - is perhaps the most multifunctional organ in the human body. First, the skeleton is the scaffold that controls the body's size and shape as we grow. Second, it provides protective structures for vital organs such as the brain, spinal cord, heart, and lung. Third, together with its associated joints and muscles, the skeleton coordinates the motion of the body in such precise ways so we can walk, dance or even play the piano. Forth, bone marrow - an inseparable part of bone - is in fact a blood organ responsible for producing the whole blood system. Fifth, every single bone in the body is a store for essential inorganic minerals like calcium, phosphate and other trace elements. What's more? Even the hollow air-filled sinuses in our skull bones are optimized to resonate acoustic waves passing through the nasal tracts, creating all those distinct vocal sounds [1].

For an organ that serves so many important roles in the body, evolution has developed mechanisms to regulate its development and maintenance throughout life. As the result, the skeleton is an incredibly dynamic and highly regenerative organ. Our daily activities create stresses and micro damages to the skeleton that get repaired all the time without us noticing. Fractured bones can often heal to functionally healthy bones with little or no clinical intervention [2]. The skeleton even continuously adapts itself in the long term to fit the body's physical demands [3]. For the majority, especially those who know the limits of their bodies, this natural automatic mode is everything we need to enjoy a great skeletal life. Even pain is good sometimes; it's the tell-tale signal that our body has reached its limit and we must rest, allowing regenerative mechanisms to kick in and restore the system.

There is one thing that evolution has not done well though: total bone regeneration or more generally, regeneration of the body. Regeneration is the key to our ability to survive disease or injury. Some people even seek immortality. Biblically, the human beings were once able to regenerate themselves - Eve was fully 'created' from the rib bone of Adam - then lost this ability, became mortal and forever sought the 'fountain of youth' or the 'elixir of life'. Many other stories exist to reflect this human dream of overcoming disease and death. Today's scientists agree that biological immortality really exists, both in nature and in the laboratory [4, 5]. The purpose of life is life itself (i.e. to produce further life), thus it makes sense that immortality is not impossible after all. The most obvious example is of course the egg cell. "The fertilised egg lives indefinitely, in as much as it gives rise, not only to a new individual, but, theoretically at least, to an endless series of generations" [6].

But that is just one cell. Could multicellular organisms can also become immortal if evolution selects the genes that make them immortal? Organisms those possess longevity genes could be selected because they can produce more offspring carrying the same genes in their lifetime. Following this route, immortal genes could have emerged. In reality, most animals have evolved to possess exactly the opposite: senescence genes that destine their bearer to aging and death [7]. 
The explanation for this is that senescence and death prevent accumulated DNA damage from weakening the organism and being passed on to future generations. In order to be immortal, the organism must also possess a sophisticated mechanism to perfectly safeguard its genome, allowing it to live long and healthy. Paradoxically, evolution by natural selection only works by allowing random DNA damages to occur; no variation equals no evolution. This is why it is difficult for immortal genes to evolve naturally.

Unsurprisingly, the human being - an intelligent creature - has never settled for what nature mercifully created for them. Like no other living creature, we dissect ourselves, perform experiments, and push the limits of our bodies. We want to fully understand all of nature's mechanisms and ultimately be able to 'play God' by turning on that manual mode that offers more enjoyable features. The human being desires to live longer and healthier while also doing things far beyond the capability of his body's design. For instance, we play more risky sports and venture to more hostile territories, all the time predisposing ourselves to accidents and injuries.

To this end, perhaps we should look back and thank our ancestors for their continuous painstaking effort in medical exploration and research. The result is that medicine is progressively getting better at fixing our health problems. But in order to archive true regeneration, the first obvious step is to understand the human body to the smallest details. The current status is very encouraging; almost $100 \%$ of the human genome has been sequenced of which $88 \%$ has been confirmed at protein level $[8,9]$. Artificial intelligent (AI) is on the rise, which promises to join forces with the human scientists to accelerate medical research at a never-seen-before pace $[10,11]$.

Genetic tools are also getting sharper and sharper; gene therapy has already been offering unprecedented treatment for formally incurable diseases such as certain cancers [12, 13]. Theoretically, the human being may eventually mend all his defected genes and even create completely new genes. This may result in a new species of human being (better or worse) and may take thousands of years more, though it is surely faster than evolution by natural selection.

In the field of bone regeneration, ever since the beginning of medicine, physicians, surgeons and scientists have been studying bone with the desire to fix bone problems such as non-union fractures, fibrous dysplasia, osteogenesis imperfecta, and osteoporosis to name just a few [14]. Lots of knowledge was obtained and numerous bone regulatory pathways have been discovered. Bone morphogenetic proteins (BMPs) for example, were discovered decades ago and are capable of inducing bone formation even in places that are otherwise difficult for bone growth and have revolutionized bone regeneration [15]. Of course, since then we have also learnt that BMPs are not the 'silver bullets' for all bone problems; in fact BMPs may even create more problems than they solve [16].

Mesenchymal stem cells (MSCs) are another example of a highly influencing discovery in the field of bone biology. Motivated by the fact that MSCs represent a novel type of progenitor cells that can differentiate in vitro into (among other lineages) the bone forming cell osteoblast, tremendous effort have been invested to characterise this cell type and utilize it for bone regenerative therapy [17]. Until now, the search for the in vivo identity of MSC is ongoing 
[18]; meanwhile, despite completion of hundreds of clinical trials the verdict for the efficacy of MSC for bone repair and regeneration is still under debate [19].

As of 2018, the widely accepted 'gold standard' treatment for bone defects and non-union is still the century-old autologous bone graft (ABG). This is not because ABG is $100 \%$ effective and without any complications, but rather it is superior to all current alternatives [20, 21]. Pragmatically speaking, bone regeneration research is still at its infancy; especially when considering the ultimate goal for bone regeneration is de novo organogenesis - for instance to regrow a limb (at least the bone part).

Just like any problem in life can be approached from at least a dozen different ways, scientists have a plethora of approaches to tackle the challenge of bone regeneration. Thus, before advancing further into complexity, it is essential to learn from the past about what has been tried, what works and what does not work. Chapter 1 of this book is dedicated to a literature review on bone components and their roles in bone regeneration. Tissue level components (periosteum, osseous tissue, endosteum and bone marrow) as well as cell and extracellular matrix components are studied.

The macroscopic tissue-level components of bone are well-familiar to medical doctors and orthopaedic surgeons, who studied and treated bone disorders long before the existence of modern scientific methods. Being the people at the bedside and delivering treatments to the patients, their clinical experience is often considered the most valuable sources of obtained knowledge to the field. Clinical knowledge such as the effect of coexisting medical conditions, pain level, periosteum, vascularization, and good fixation are often overlooked by non-clinical research scientists. Such knowledge is indispensable for the successful implementation of novel therapies and must be considered at the very beginning of any research approach.

On the other hand, laboratory scientists spearhead the development of disrupting technologies offering unprecedented therapeutic options. As mentioned above, the discovery and use of BMPs and MSCs significantly stirred up interests in both the research and industrial community for the development of better bone healing/regenerating therapies. Such major advances are only achievable through basic research, following years of painstakingly isolating every biological component and testing them using sophisticated laboratory equipment and methods.

Chapter 1 also gives an overview of bone composition from a microscopic point of view, the cellular and extracellular matrix (ECM) component of bone, and their utilization in bone tissue engineering. The cells in bone, as will be discussed, are the most valuable resource for bone tissue-engineers, but like all human resources, it is difficult to control them in order to obtain the desired results. Likewise, great progress has been made to characterize the many components of bone ECM. Numerous bone substitute materials have been developed and used in the clinic to mimic human bone ECM, and thus trying to achieve osteoconductivity - the ability to propagate bone formation - of an autologous bone graft. However there is little consensus on the standard-of-care bone graft substitute material for bone regeneration. The search for the best bone graft substitute continues. 
In chapter 2, the focus is on collagen, an important ECM protein for structural support in all animal tissues and in particular bone. The lack of collagen, as in osteogenesis imperfecta - also known as brittle bone disease, results in severely fragile bones. Bone formation starts with osteoblasts secreting type I collagen (among other specialized proteins in smaller amounts) to form the organic matrix of bone, which then be impregnated with hydroxyapatite to become the strong and dense mineralized bone tissue. One way of increasing bone volume and strength could be to increase collagen production by the cells, which is the inspiration for the work in this chapter. Using a conventional high throughput screening approach, we attempted to search for a compound from a LOPAC library containing 1280 pharmacologically active drugs, which could induce collagen production from cells growing in vitro on culture plates.

Aiming to target the cells involved in endochondral ossification, the process occurring during growth and fracture healing of bone, we chose the ATDC5 cell line to perform this screen. ATDC5 is a very interesting cell line because it can fully recapitulate the chondrogenic stages of endochondral ossification [22]. For the same reason, ATDC5 cells will also appear in chapter 2 and 3 of this book. When cultured under certain stimulation - in this case a chemical stimulus such as insulin - ATDC5 cells can rapidly proliferate and form cell clusters similar to the process of mesenchymal condensation. Within these clusters, cells start producing collagen to build a thick ECM encapsulating themselves into dense nodules.

To assess the amount of this collagenous ECM after the cells had been stimulated by various different compounds, we used a special collagen probe (CNA35) conjugated to a fluorescent dye (Alexa Fluor 488). The probe CNA35 possesses two collagen binding domains from Staphylococcus aureus bacteria which use them to anchor to the host ECM; CNA35 recognizes the collagen triple helix and wraps itself around the collagen molecule [23, 24]. This binding mechanism allows the probe to bind to a variety of collagen types, thus increasing the assay's sensitivity while scarifying its specificity. Using this set-up, we discovered 13 compounds from the library that induced collagen production from ATDC5 cells; of which 1 compound tetradecylthioacetic acid (TTA) - performed consistently in subsequent validation tests. We also tried using TTA to induce collagen production in other cells types, namely human MSCs and human primary chondrocytes; however the same effect was not observed in these cell types. Thus, despite having a collagen inducing effect on ATDC5 cells, TTA needs to be further investigated to elucidate its mechanism of action.

Next, in chapter 3, we continue the in vitro production of cartilaginous ECM from ATDC5, but taking a 3D approach to culture the cells. The work in this chapter was inspired by an old publication by Bridge and Pritchard in 1958 [25]. In this report, they showed that hypertrophic cartilage such as the tissue of fracture callus and growth plate can form bone when implanted in the ears of rabbits, even after devitalization, a treatment to kill all living cells. It suggested that the ECM of hypertrophic cartilage contains an appropriate composition of collagen matrix, chemokines and growth factors to trigger the bone forming cascade. Now we all know that a lot of cell-free materials can augment bone regeneration including a wide range of inorganic calcium phosphate minerals, so a devitalized hypertrophic ECM inducing bone formation is nothing startling in itself. It is just intriguing that some components of the hypertrophic cartilage matrix, such as collagen type $\mathrm{X}$, are rarely evaluated for bone regeneration. 
In order to follow this interesting approach, one must have a platform to manufacture sufficient quantity of hypertrophic cartilage since it is impractical to harvest the tissue from fracture callus or growth plate of lab animals. My senior supervisor once suggested to collect this tissue from African elephants (the young ones), but that project never started. Then came the idea of growing hypertrophic cartilage in vitro from cells. One could use stem cells such as MSCs or even embryonic stem cells to produce hypertrophic cartilage, following a number of differentiation protocols to guide the cells into chondrogenic lineage. However this route is incredibly expensive considering the cost of stem cell maintenance and growth factors involved in differentiation, not to mention its low efficiency. For the purpose of upscaling in vitro production of hypertrophic cartilage, we again chose ATDC5 because of their robustness and more importantly the fact that ATDC5 is a chondrogenic cell line with designated fate to undergo hypertrophy with almost minimal stimulation [22, 26].

Although ATDC5 are commonly cultured on a flat surface while inducing the chondrogenic pathway, we have shown in this chapter that growing them in small cell aggregates (micro tissue-engineered cartilage - MiTEC) significantly improves the quantity and quality of its hypertrophic cartilage ECM. The cell aggregates were formed from approximately 1000 cells, much less than common cell pellets of 0.1-0.5 million cells, allowing the formation of a thick extracellular matrix without interrupting nutrition supply and waste exchange with the culture medium. To demonstrate the efficacy of the system, we tested various medium compositions to induce hypertrophic differentiation, mineralization and even stimulate growth factor (vascular endothelial growth factor - VEGF) production using small molecules. As a proof of concept, we devitalized or decellularized the aggregates with either liquid nitrogen or detergent, and then co-cultured them with human MSCs to see the effect of the cell-free matrixes on the stem cells. The ultimate goal for this approach is to achieve high throughput production of hypertrophic cartilage matrix in vitro, and then to optimize decellularization and isolation of individual matrix components, which comprise novel material types for bone regeneration.

While the approaches in chapter 2 and 3 require nothing more than an ordinary tissue culture laboratory and some common molecular analytic equipment, the one in chapter 4 is a giant leap in technological involvement. To start with, the question was simple. Can certain micro/nano-topography on the material surface influence cells to do certain things, similar to growth factors directing cell fate? Assuming the answer is yes, then how are we going about finding the exact type of topography that can target a particular pathway in our cells of interest?

One way of tackling this is to generate a great number of varying topographies and perform a high-throughput screen, relatively similar to traditional drug screening. The challenge lies in the generation of screening libraries containing thousands of random micro/nano-scale surface topographies. This is when a huge laboratory with cleanrooms, robotic arms, and sophisticated (and expensive) photolithographic equipment is needed. Previously, our group has generated one such topographical library; it is called the TopoChip, containing 2176 distinct surface patterns, in duplicate, all fitting in a $2 \times 2 \mathrm{~cm}$ chip [27].

In one high-throughput screen of the TopoChip, it was discovered that some topographies can induce the production of the enzyme alkaline phosphatase (ALP) from human MSCs. Since 
ALP is also upregulated, and thus a marker, at the beginning of hypertrophic differentiation of ATDC5, we wanted to test these same topographies on ATDC5 cells. In order to use conventional molecular methods such as PCR, we applied the patterns on the larger surface of 12 -well culture plates. ATDC5 cells grown on these topographical surfaces were then compared to the ones grown on a flat coverslip.

Indeed there was an effect of the topographies on ATDC5 growth and differentiation. The patterns that induce ALP production in human MSCs also seemed to boost hypertrophic differentiation in ATDC5. Although many questions remained unanswered, this was another proof-of-concept experiment to demonstrate that topographical cues, just like chemical and mechanical cues, can be utilized to direct cellular development. The final aim in this approach is to decipher the cell's response to surface topography, and eventually be able to create instructive topography on the surface of medical implants or in vitro culture dishes. Without a doubt, the benefit of this will extend far beyond just bone regeneration purpose.

Finally, in chapter 5 of this book, we returned to use one of the most fundamental approaches in biology, a genetic approach. Inspired by the emergence of the sharpest genome editing tools ever developed - the CRISPR-Cas9 complex - we decided to at least try it out for ourselves, knowing that a genome editing project is a very challenging one. There are so many advantages that genome editing can offer. One can fix the root of a problem forever by editing a defective gene. One can also have the opportunity to explore the root of knowledge, understand the ultimate function of a particular gene, for instance by permanently fusing a reporter to the gene of interest. In this chapter, we endeavoured to devise a stable reporter cell line for the famous bone growth factor BMP-2.

At the time of writing, there are a few existing BMP-2 knockout mouse models, such as the ones by Tsuji et al in 2008 [28] or Wang et al in 2011 [29], both are very useful in demonstrating the indispensable function of BMP-2 in skeletal development and repair. However, there has not been any report of a stable BMP-2 reporter cell line to date; that is to say our attempt to generate one was also not successful. Such BMP-2 reporter cell line could help elucidate the involvement of the BMP-2 signalling pathway in various cellular responses such as cell-material interaction. The cell line could also be used in many different screens for factors that can induce or supress BMP-2 expression. Despite the failure, we have reached as far as having the complete homologous sequence of the modified BMP-2 gene with its fused GFP reporter, which is a quest on its own since the 3'-UTR region of BMP-2 is notoriously difficult to clone. With the rapid evolution of genome editing tools (CRISPR has just been upgraded to version 2.0), it is likely that a BMP-2 reporter cell line will soon be available.

Overall, of the plethora of approaches that researchers can choose to tackle the bone regeneration challenge, it is unlikely that one will definitely be better than others. Clinically it is well-understood that not all patients respond similarly to the same treatment. There is a long way to go in order to understand the human body as a whole and to devise the best therapy tailored to each individual. Until then, the scientist's job is to explore as many approaches as possible, revisiting old approaches as often as necessary, and trying difficult approaches even when everyone has failed doing so. 


\section{References for Introduction}

1. Weiner, S. and H.D. Wagner, The material bone: structure-mechanical function relations. Annual Review of Materials Science, 1998. 28(1): p. 271-298.

2. Victoria, G., et al., Bone stimulation for fracture healing: What's all the fuss? Indian J Orthop, 2009. 43(2): p. 117-20.

3. Burt, L.A., D.A. Greene, and G.A. Naughton, Bone Health of Young Male Gymnasts: A Systematic Review. Pediatr Exerc Sci, 2017. 29(4): p. 456-464.

4. Ruby, J.G., M. Smith, and R. Buffenstein, Naked Mole-Rat mortality rates defy gompertzian laws by not increasing with age. Elife, 2018. 7.

5. Shay, J.W. and W.E. Wright, Hayflick, his limit, and cellular ageing. Nat Rev Mol Cell Biol, 2000. 1(1): p. 72-6.

6. Overton, J.B., Artificial parthenogenesis in Fucus. Science, 1913. 37(961): p. 841-844.

7. Nussey, D.H., et al., Senescence in natural populations of animals: widespread evidence and its implications for bio-gerontology. Ageing Res Rev, 2013. 12(1): p. 214-25.

8. International Human Genome Sequencing, C., Finishing the euchromatic sequence of the human genome. Nature, 2004. 431(7011): p. 931-45.

9. Baker, M.S., et al., Accelerating the search for the missing proteins in the human proteome. Nat Commun, 2017. 8: p. 14271.

10. Schneider, G., Automating drug discovery. Nat Rev Drug Discov, 2017.

11. Grace, K., et al., When Will AI Exceed Human Performance? Evidence from AI Experts. arXiv preprint arXiv:1705.08807, 2017.

12. Naldini, L., Gene therapy returns to centre stage. Nature, 2015. 526(7573): p. 351-60.

13. Keeler, A.M., M.K. ElMallah, and T.R. Flotte, Gene Therapy 2017: Progress and Future Directions. Clin Transl Sci, 2017. 10(4): p. 242-248.

14. Donati, D., et al., Bone grafting: historical and conceptual review, starting with an old manuscript by Vittorio Putti. Acta Orthop, 2007. 78(1): p. 19-25.

15. Gautschi, O.P., S.P. Frey, and R. Zellweger, Bone morphogenetic proteins in clinical applications. ANZ J Surg, 2007. 77(8): p. 626-31.

16. Tannoury, C.A. and H.S. An, Complications with the use of bone morphogenetic protein 2 (BMP-2) in spine surgery. Spine J, 2014. 14(3): p. 552-9.

17. Ullah, I., R.B. Subbarao, and G.J. Rho, Human mesenchymal stem cells - current trends and future prospective. Biosci Rep, 2015. 35(2).

18. Caplan, A.I., Mesenchymal Stem Cells: Time to Change the Name! Stem Cells Transl Med, 2017. 6(6): p. 1445-1451.

19. Knight, M.N. and K.D. Hankenson, Mesenchymal Stem Cells in Bone Regeneration. Adv Wound Care (New Rochelle), 2013. 2(6): p. 306-316.

20. Pape, H.C., A. Evans, and P. Kobbe, Autologous bone graft: properties and techniques. J Orthop Trauma, 2010. 24 Suppl 1: p. S36-40.

21. Sakkas, A., et al., Autogenous bone grafts in oral implantology-is it still a "gold standard"? A consecutive review of 279 patients with 456 clinical procedures. Int J Implant Dent, 2017. 3(1): p. 23.

22. Yao, Y. and Y. Wang, ATDC5: an excellent in vitro model cell line for skeletal development. J Cell Biochem, 2013. 114(6): p. 1223-9.

23.Zong, Y., et al., A 'Collagen Hug' model for Staphylococcus aureus CNA binding to collagen. EMBO J, 2005. 24(24): p. 4224-36.

24. Aper, S.J., et al., Colorful protein-based fluorescent probes for collagen imaging. PLoS One, 2014. 9(12): p. e114983.

25. Bridges, J.B. and J.J. Pritchard, Bone and cartilage induction in the rabbit. J Anat, 1958. 92(1): p. 28-38. 
26. Shukunami, C., et al., Chondrogenic differentiation of clonal mouse embryonic cell line ATDC5 in vitro: differentiation-dependent gene expression of parathyroid hormone (PTH)/PTH-related peptide receptor. J Cell Biol, 1996. 133(2): p. 457-68.

27. Unadkat, H.V., et al., An algorithm-based topographical biomaterials library to instruct cell fate. Proc Natl Acad Sci U S A, 2011. 108(40): p. 16565-70.

28. Tsuji, K., et al., BMP4 is dispensable for skeletogenesis and fracture-healing in the limb. J Bone Joint Surg Am, 2008. 90 Suppl 1: p. 14-8.

29. Wang, Q., et al., Expression of endogenous BMP-2 in periosteal progenitor cells is essential for bone healing. Bone, 2011. 48(3): p. 524-32. 


\title{
Chapter 2
}

(Review)

\section{The components of bone and what they can teach us about regeneration}

\author{
Bach Q. Le, Victor Nurcombe, Simon M. Cool, Clemens A. van \\ Blitterswijk, Jan de Boer, and Vanessa L.S. LaPointe
}

$\mathrm{T}$

he problem of bone regeneration has engaged both physicians and scientists since the beginning of medicine. Not only can bone heal itself following most injuries, but when it does, the regenerated tissue is often indistinguishable from healthy bone. Problems arise, however, when bone does not heal properly, or when new tissue is needed, such as when two vertebrae are required to fuse to stabilize adjacent spine segments. Despite centuries of research, such procedures still require improved therapeutic methods to be devised. Autologous bone harvesting and grafting is currently still the accepted benchmark, despite drawbacks for clinicians and patients that include limited amounts, donor site morbidity and variable quality. The necessity for an alternative to this "gold standard" has given rise to a bone-graft and substitute industry, with its central conundrum: what is the best way to regenerate bone? In this review, we dissect bone anatomy to summarize our current understanding of its constituents. We then look at how various components have been employed to improve bone regeneration. Evolving strategies for bone regeneration are then considered. 


\section{Introduction}

The human skeletal system consists of 206 bones and provides a rigid support for every other organ in the body. Some organs, like the brain and spinal cord, are protected inside bone structures - the skull and vertebrae, respectively. Other organs, such as the muscles, are attached to the skeleton. The skeleton is an adaptive structure, and as it grows through childhood, the rest of the body grows along with it. Apart from providing structure and protection, the skeletal system functions to cooperate with joints and muscles for movement. Other critical functions of the skeletal system include blood cell production, mineral storage and endocrine regulation [1].

Throughout life, the skeletal system has to endure great physical stress, predisposing it to injuries and disorders. Fortunately, the body has developed superb mechanisms to adapt and regenerate bone. For example, bone strength can increase in response to weight gain or following athletic training [2], and with minimal clinical intervention, fractured bones can heal into functionally normal bone [3]. Unfortunately, in 5-10\% of cases where healing is compromised, the economic and health burden is significant. The Global Burden of Disease study (2013) found musculoskeletal conditions such as arthritis and back pain affect more than 1.7 billion people worldwide. These conditions are the leading cause of years lived with disability in 86 countries, and the second or third leading cause in 67 countries [4].

Three major breakthroughs have fueled recent advances in bone research. First, the discovery of bone morphogenetic proteins (BMPs) in 1965 initiated a new era of research and development for bone growth factor therapy [5]. The discovery of mesenchymal stem cells (MSCs) in 1991, coincident with the first isolation of human embryonic stem cells, also stimulated significant interest [6]. Finally, the development of materials mimicking bone extracellular matrix, including calcium phosphate ceramics, collagens, and glycosaminoglycans, exponentially increased the number of available alternatives to bone graft [7]. With this, the concept of a tissue engineering "triangle" consisting of growth factors, cells and scaffolds has continued to provide a growing list of bone graft substitutes.

For those trying to develop bone graft substitutes through biomimicry, it is important to thoroughly and diligently study bone and its constituents. In this review, we consider the most current and salient facts pertaining to skeletal healing. We review the constituents of bone and the contributions each component makes to bone healing. And we ask, what has been tested and what does and does not work when used for treating bone injuries?

Structure of the review

2. Dissecting bone at the tissue level

2.1. Periosteum

2.2. Osseous tissue

2.3. Endosteum

2.4. Bone marrow

3. The cells of bone

4. The extracellular matrix of bone

5. The future of bone tissue engineering 


\section{Dissecting bone at the tissue level}

\subsection{Periosteum}

The outermost layer of almost every bone in the body comprises the periosteum, a dense bilayer membrane responsible for appositional bone growth in children, partial blood supply to bone, and bone fracture repair (Fig 1). Periosteum consists of two distinct layers, an outer fibrous layer providing structural support, and an inner cambium layer abundant in osteoprogenitor cells. As early as the 18th century, surgeons discovered that periosteum itself had the potential to induce new bone formation $[8,9]$. Today, surgeons take care not to disturb or remove the periosteum around sites of injury as they believe it is one of the most crucial components of bone healing [10]. Despite this important function, periosteum receives less attention from scientists than other bone components.

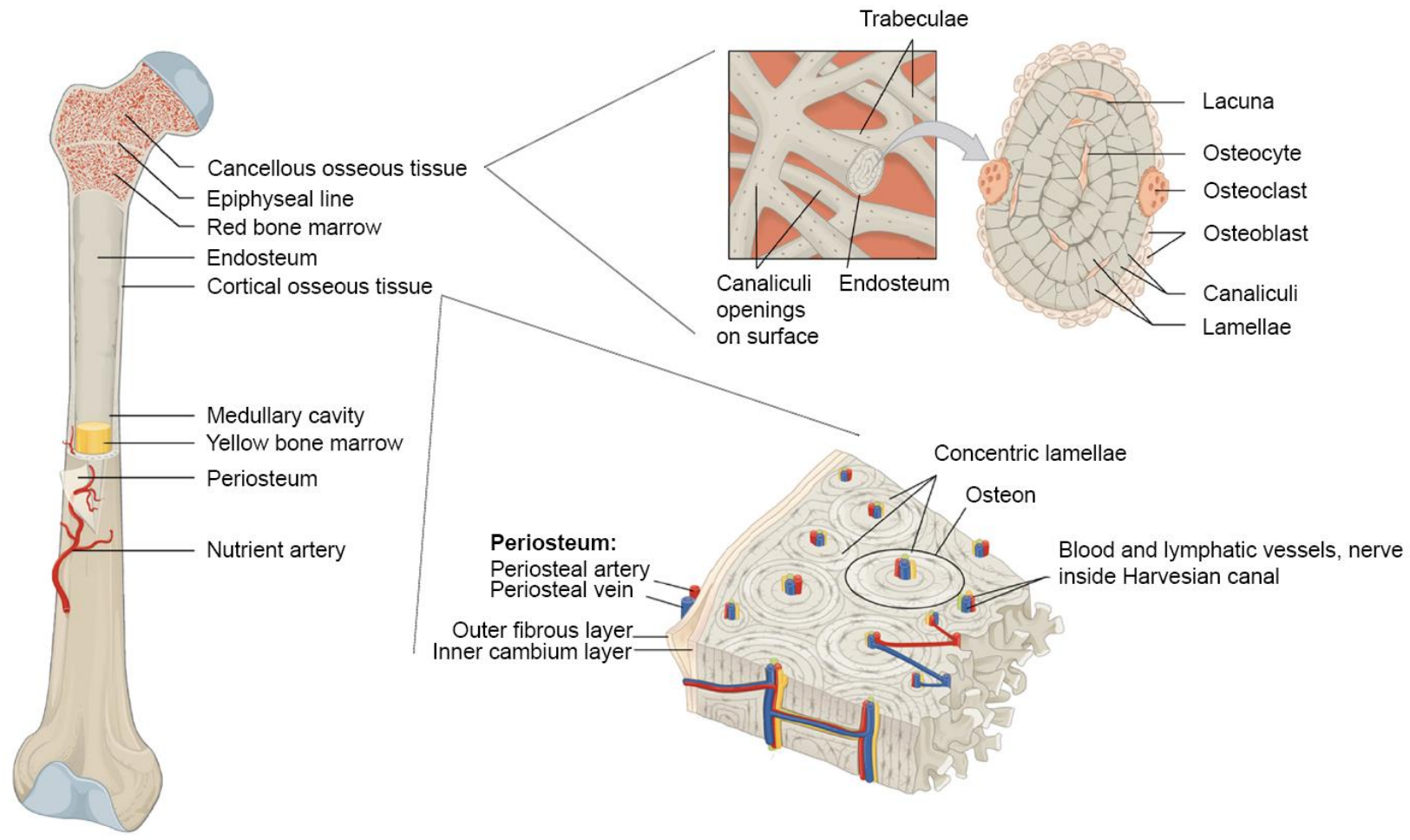

FIG. 1. Bone anatomy. Modified and combined from Wikimedia Commons by OpenStar College CC BY 3.0 license [11].

The idea of utilizing a periosteal graft to augment bone healing at distant sites is not novel, but the problem with transplanting periosteum is that its blood supply must be maintained to keep the osteogenic cells viable. In the 1990s, Doi and Sakai demonstrated the use of vascularized periosteal flaps, consisting of a thin flap of periosteum with its intact blood supply, to cover bone defects [12]. This method has been used successfully to treat difficult cases of large bone defects and persistent pseudarthrosis [13-15]. An interesting experiment by Saito et al. tested the osteogenic capacity of vascularized periosteum by wrapping it around beta- 
tricalcium phosphate (b-TCP) before implantation in rabbit muscle [16]. Bone formation was observed in the group with vascularized periosteum, but incomplete osteogenesis was found if the periosteum was non-vascularized, and no bone was found if b-TCP alone was implanted. This experiment emphasized the importance of a blood supply to the periosteal graft to successfully direct bone repair in vivo.

In addition to the need for vascularization, another issue with periosteum is the thickness of its inner cambium osteoprogenitor layer decreases with age, concomitant with a decrease in its osteogenic potential [17]. The cells responsible for the osteogenic potential have been shown to be MSCs, and despite being more difficult to harvest, periosteal MSCs have been shown to be a better healing source than MSCs harvested from bone marrow [18, 19]. Intriguingly, MSCs from periosteum possess unique chondrogenic potential, as demonstrated by the major contribution they make to the cartilaginous callus during fracture healing. Indeed, removal of the periosteum, but not the bone marrow or endosteum, inhibits cartilage formation and endochondral ossification [20]. In addition to the decreased osteogenic capacity with ageing, the anatomic location of the cells appears to be important. Studies have shown that tibial periosteum has more osteogenic potential than that of the calvaria [21, 22].

As with many tissues, the limited availability of periosteum hinders its use as an autograft in multiple or large bone defects. As such, attempts have been made to engineer constructs that mimic periosteal functions for bone regeneration [23-26]. Baldwin et al. recently created an orthotopic xenograft model to evaluate their tissue-engineered periosteum, a construct that included osteoprogenitor and vascular compartments [27]. Their multiphasic construct combined a star-polyethylene glycol (PEG) heparin hydrogel system loaded with human umbilical vein endothelial cells (HUVECs) with a poly( $\varepsilon$-caprolactone) tubular scaffold seeded with human bone marrow-derived MSCs. After 30 days in vivo, the transplanted MSCs in the hydrogel layer retained their undifferentiated phenotype and the HUVECs developed into mature functional vessels connected to the host vasculature. Their results confirmed that the periosteum is a promising tissue that can be used to regenerate bone effectively if and only if it is well-vascularized.

\subsection{Osseous tissue}

Osseous tissue is the rigid mineralized tissue that accounts for the structural aspect of skeletal function, and can, therefore, be said to define bone. Formally, osseous tissue is that which is enveloped between the periosteum and the endosteum, excluding the bone marrow. Previously it was thought that osseous tissue lacked osteoprogenitor cells and that only osteocytes were present, apart from vascular cells inside Haversian canals [28]. Recently, MSCs have been isolated from osseous tissue using collagenase digestion and have been demonstrated to be essentially equivalent to bone marrow-derived MSCs [29, 30].

Osseous tissue of cortical bone and cancellous bone are biochemically identical but structurally distinct. It consists of an organic phase of mainly collagen fibers, which impart strength, flexibility, and resistance to torsional force, and an inorganic phase of mainly hydroxyapatite, which provides resistance to compression. Structurally, cortical osseous tissue 
can be described as a solid containing a series of voids (Haversian canals, Volkmann's canals, lacunae, and canaliculi) with an overall porosity of $\sim 10 \%$. Cancellous osseous tissue, on the other hand, is a network of small, interconnected plates of trabeculae with relatively large spaces between them, having a porosity between 50-90\% [31]. Osseous tissue is the primary component in many bone graft materials including autogenous, allogeneic (fresh frozen, freezedried and/or demineralized), and xenogeneic (irradiated, deproteinized and/or demineralized) bone grafts. However, to isolate the effect of osseous tissue from other tissues, one would need to use either cortical bone without the periosteum or processed cancellous bone that does not contain bone marrow. Under such conditions it has been realized that osseous tissue alone is not only a good osteoconductive material, it also possesses slightly osteoinductive activity, especially when used fresh [32].

Cortical bone contains significantly more osseous tissue per unit weight than cancellous bone, and thus is better at providing the initial mechanical stability and strength for bony defects. However, the structure of this dense cortical osseous tissue becomes a barrier to vascular ingrowth and remodeling [33, 34]. An exception to this is the autologous vascularized cortical (e.g. fibular) bone graft that has both superior mechanical properties and speed of healing [35]. Cancellous bone provides little initial mechanical strength, but its porous structure allows rapid invasion by blood vessels and hence faster remodeling. In practice, physicians control the balance between initial mechanical strength and remodeling/healing speed by adjusting the ratio of cortical/cancellous bone they use [36].

The osteoconductive properties of osseous tissue have been leveraged to create scaffolds that maximize bone remodeling capacity. Bone cells such as osteoclasts, macrophages and osteoblasts naturally form basic multicellular units on the surface of osseous tissue, following specific remodeling signals to repair structural damage or respond to changes in mechanical loading [37]. Without vasculature, an osseous tissue graft quickly becomes necrotic, such that the whole graft must be remodeled - resorbed and replaced with new osseous tissue - for healing to be completed [38]. The mineralized matrix of osseous tissue also harbors abundant growth factors including IGFs and BMPs that have mitogenic and osteogenic activity [39], suggesting that osseous tissue is also osteoinductive. The benefit of this growth factor reservoir depends on how the osseous tissue is processed, because this affects growth factor stability, availability and bioactivity. Autologous osseous tissue is often minimally processed and used fresh, so maintaining its full osteoinductive potential. Allogeneic and xenogeneic osseous tissue in contrast must be processed to eliminate the immunogenicity that causes graft rejection. Demineralized bone matrix (DBM) is osseous tissue processed to remove all cells and almost all minerals; what remains is an organic extracellular matrix that has been shown to be abundant in growth factors [40]. Nevertheless, it is not easy to process osseous tissue while preserving the endogenous growth factors. Different methods combined with variable starting materials results in inconsistent end products [41]. Despite the number of versions on the market, the efficacy of DBM for bone healing has recently been questioned [42, 43]. However, taken together, osseous tissue is an excellent scaffold for bone regeneration, though its full osteogenic potential depends on the source of the tissue and the processing methods. Osseous tissue has been, and is currently 
used in combination with other bone components such as cells and bone growth factors to achieve better overall grafting performance.

\subsection{Endosteum}

Endosteum is the least studied component of bone. A PubMed search for titles containing "endosteum" or "endosteal" surprisingly only yields 446 results at the time of writing; this compares to 2,559 for "periosteum" or "periosteal", 6,091 for "osseous", and 63,165 for "bone marrow" (Table 1). Endosteum arises from periosteum that becomes entrapped during appositional bone growth. As periosteal osteoblasts form new bone matrix on the bone surface, part of the periosteum and its vasculature is slowly engulfed in newly formed osteons. The blood vessels become the Haversian blood vessels that nurture osseous tissue, while the periosteum becomes the endosteum lining Haverisian canals and medullary cavities [44]. Unlike the periosteum, the endosteum is a very thin membrane, averaging only $10-40 \mu \mathrm{m}$ in thickness, consisting of an indistinct connective tissue layer and a few layers of cells [45]. The cells in endosteum are arranged in a mosaic pattern of formative, resting and resorptive areas, characterized by the presence of active osteoblasts, preosteoblasts or osteoclasts respectively [46]. Functionally, endosteum contributes to bone repair and reconstruction just as much as the periosteum, as it houses osteoprogenitor cells such as MSCs and preosteoblasts [47]. Endosteum also serves to control the weight-to-strength ratio of long bones by resorbing unnecessary osseous tissue from the medullary cavity as bones are growing in thickness. Because of its thin and indistinct appearance, endosteum as a tissue is less favored by orthopedic surgeons than other bony components. As endosteum originates from periosteum, it would appear to be more logical to use the periosteum, which is thicker, easier to handle, replete with its own blood vessels, and contains more osteoprogenitor cells. The resorptive capability of endosteum may be more useful in controlling bone overgrowth or even undesirable ectopic bone formation. However, this ability, largely uncharacterized and poorly understood, is the result of careful adjustments of the bone remodeling signaling system, tilting the balance toward controlled bone resorption. In short, the endosteum on its own has not been recognized as a suitable source for tissue engineering of grafts for bone healing and regeneration. Despite that, the complex molecular mechanism resulting in the bone resorptive property of endosteum clearly warrants further investigation.

\subsection{Bone marrow}

Bone marrow is one of the vulnerable soft tissues that is protected inside bone. Despite being part of bone anatomy, bone marrow is better characterized by its function as a blood organ. Of the 63,165 hits on PubMed with "bone marrow" in [title] (the search term not including "mesenchymal stem cells"), only 805 hits can be found to couple with either "bone regeneration", "bone healing", "bone tissue engineering", "fracture healing" or "non-union" in [title/abstract], accounting for only $1.3 \%$ of all bone marrow-related studies (Table 1). As a blood organ, bone marrow has been well established as the key niche for hematopoietic stem cells, which can regenerate the whole blood system from as little as one cell [48]. 
Concomitantly, bone marrow is also part of the bone organ as long bone, and its marrow almost always coexist spatially and temporally. To this end, bone marrow has been postulated to be the niche for another stem cell type, the MSCs, although this matter is still under some dispute [4951].

Table 1. Summary of terms used in the PubMed search conducted in October, 2017.

\begin{tabular}{lll}
\hline Tissue & Search term & $\begin{array}{l}\text { Number } \\
\text { of hits }\end{array}$ \\
Periosteum & Periosteum [Title] OR periosteal [Title] & 2,559 \\
\hline Osseous & Osseous [Title] & 6,091 \\
\hline Endosteum & Endosteum [Title] OR endosteal [Title] & 446 \\
\hline Bone marrow & "bone marrow" [Title] & 63,165 \\
\hline $\begin{array}{l}\text { Bone marrow } \\
\text { (related to }\end{array}$ & ("bone marrow" [Title]) AND (("bone regeneration" [Title/Abstract]) & 805 \\
bone research) & OR ("bone healing"[Title/Abstract]) OR ("bone tissue engineering" & \\
& [Title/Abstract]) OR ("fracture healing" [Title/Abstract]) OR & \\
\hline
\end{tabular}

It is now unquestionable that bone marrow has its uses for bone regeneration. Early studies showed its osteogenic potential when fragments of bone marrow or bone marrow cell suspensions were implanted into various ectopic sites in animals, including subcutaneous, and resulted in de novo bone formation [52, 53]. It was noted that even if the sample of bone marrow was small enough to avoid rapid necrosis, bone formation only occurred in the zone of outgrowing cells, implying that the marrow structure is incompatible with bone formation. Indeed, bone marrow therapy today is essentially a cell therapy. Kuznetsov et al. showed that populations of stromal fibroblast cells isolated from human bone marrow could differentiate into osteoblasts, and form bone when subcutaneously implanted into immunodeficient mice [54]. Interestingly, while new bone was formed by the human cells, new bone marrow was colonized by the host's cells. Until now, a great number of in vivo studies, mostly in laboratory animals, support the use of bone marrow for bone healing and regeneration [55]. On the other hand, the evidence from clinical studies has as yet been insufficient to demonstrate significantly improved bone healing when using bone marrow aspirate or bone marrow stem cells for conditions such as long bone fractures [56]. Nonetheless, bone marrow is generally believed to improve the outcome of bone healing, which is usually ascribed to its high stem cell content. Indeed, the osteogenic potential of a bone marrow aspirate directly correlates to the number of osteogenic stem cells it contains [57]. This, in turn, depends on how the aspirate is harvested, which further contributes to the variable results reported in the literature [58]. To sum up, bone marrow tissue undoubtedly possesses one of the key components for successful bone regeneration: the stromal stem cells. This resource, however, is among the most difficult to be utilized, as will be discussed in the next section. 


\section{The cells in bone}

Bone cells can be categorized into two lineages: the osteoblast lineage, representing the bone-forming axis (consisting of MSCs, pre-osteoblasts, mature osteoblasts, bone-lining cells and osteocytes), and the osteoclast lineage, representing the bone-resorbing arm (consisting of macrophages, osteoclasts and multinucleated giant cells, all derived from bone marrow haematopoietic stem cells). The balance between bone-forming and -resorbing is modified throughout life to attain and preserve skeletal size, shape and structural integrity.

Bone-forming cells are, understandably, the focus of bone tissue engineers. Mature osteoblasts are the only cells that can explicitly build bone by secreting bone matrix proteins and guiding mineralization. Depletion of mature osteoblasts results in an arrest of skeletal growth [59]. However, mature osteoblasts are short-lived; a subset is encapsulated within the newly formed bone matrix, becoming osteocytes, while the others either undergo apoptosis or become inactive bone-lining cells [60]. During bone healing, osteoblasts are continuously replenished from the osteogenic cells, pre-osteoblasts and MSCs residing in such nearby bone compartments as the bone marrow, endosteum and periosteum [61]. In fact, MSCs are critical to the bone-healing process as the precursors of both osteoblasts (involved in intramembranous ossification) and chondrocytes (involved in endochondral ossification). Additionally, MSCs can secrete numerous trophic factors to establish and regulate a regenerative microenvironment, for which they have recently earned the epithet 'in vivo drugstore' [51]. Various regulatory pathways such as the Indian hedgehog (IHH), Notch, WNT, BMP and FGF signaling pathways are involved in the differentiation of MSCs into osteoblasts or chondrocytes, although much remains unknown. There are also many questions related to MSCs in vivo that are still extant, foremost their origin $[62,63]$.

MSC therapy for bone regeneration has gained enormous interest in the last decade thanks to the growing understanding of the role of bone stem cells in healing and the general methodological readiness for such experiments. MSCs have been isolated from various tissue sources (including bone marrow, periosteum, adipose tissue, dental pulp, and umbilical cord) and can be cultured in vitro to clinically-sufficient numbers (hundreds of millions of cells). They can even be primed to improve their in vivo survival and performance [64, 65]. Importantly, clinical trials involving MSCs have demonstrated that such therapy is safe, with few detrimental effects [66]. Abundant proof-of-concept studies using MSCs in combination with various types of carriers and delivery methods have demonstrated efficacy for bone regeneration in different animal models [67-70]. However, many outcomes of the manipulation of MSCs for bone regeneration are inconclusive despite the increasing number of registered clinical trials $[65,71,72]$. One particular exception is the trials using allogeneic MSCs for the bone disease osteogenesis imperfecta (OI), also known as 'brittle bone' disease, in which the patients lack a functional type I collagen gene. Here, MSC therapy has been initially successful, especially given the severity and lack of an effective cure for the disease [73, 74]. It is also worth noting that less than $10 \%$ of all MSC clinical trials are directly related to bone and cartilage disorders; the other $90 \%$ have been for cardiovascular disorders, autoimmune disease, liver disease, kidney disease, and skin disease, amongst others [65]. 


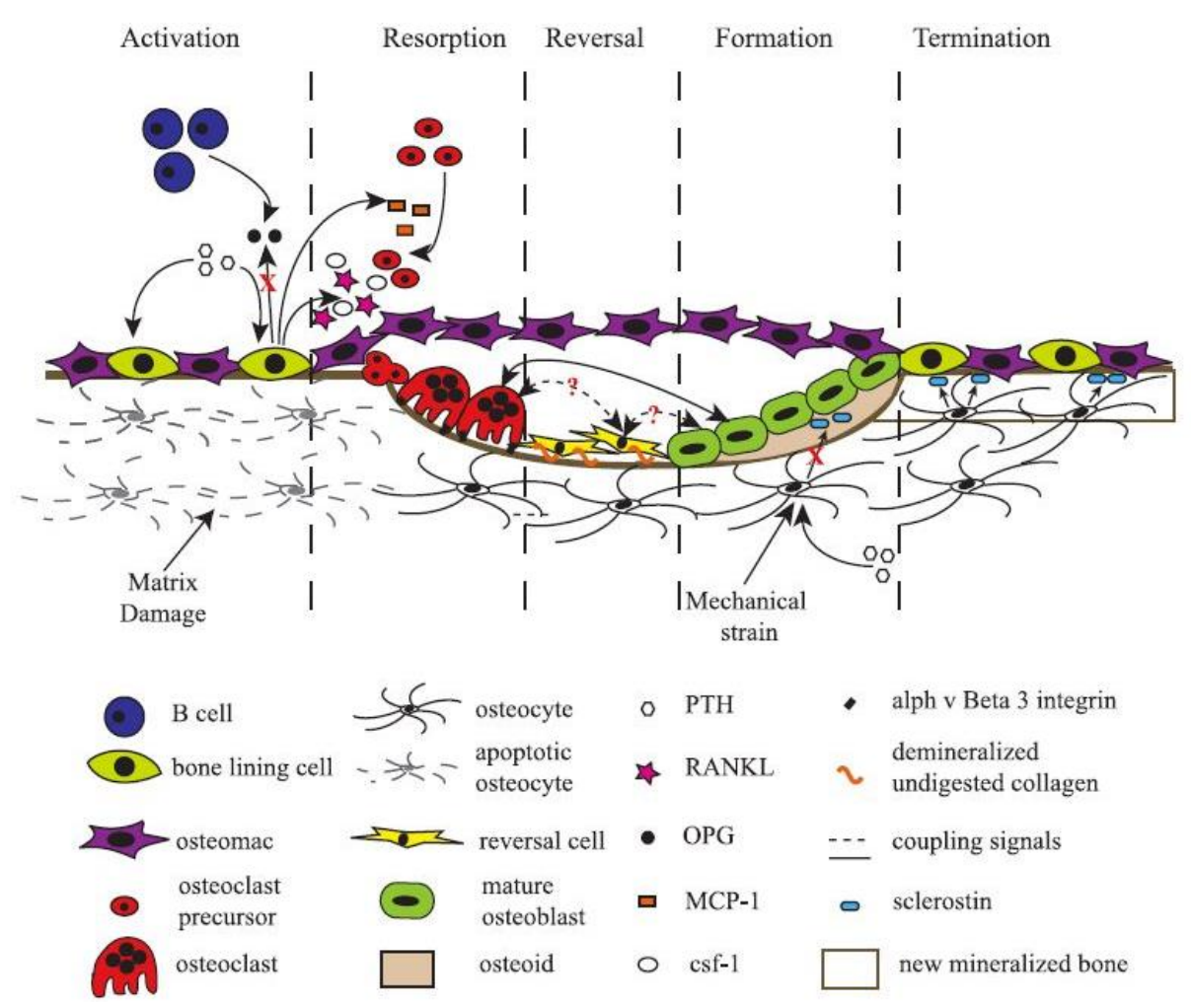

FIG 2. Schematic representation of a basic multicellular unit and the associated bone remodeling process. Permission obtained from ASBMB. (C) Liza J. R. and Nicola C.P. J. Biol. Chem. 285: 25103 (2010). [37]

More recently, scientists have turned their attention to another cell type found in bone tissue, the bone-resident macrophage, termed "osteomac" by Pettit et al. in 2013 [75-77]. Originally thought to only function as immune surveillance in the bone environment, osteomacs have recently been demonstrated to be indispensable for bone formation. Depletion of macrophages, and thereby osteomacs, results in a complete loss of mature osteoblasts and impaired bone formation [78-80]. Bone-forming cells only function in a specific micro-environment that is carefully laid down by osteoclasts, bone-lining cells and osteomacs [37]. Osteomacs are the first cells to arrive at injury sites and interact with the foreign bodies [81]. They have been shown to form a distinct canopy-like cell structure that encapsulates functioning osteoblasts, thereby creating a specific working micro-environment for bone remodeling (Figure 2). Still, much remains unknown about the role, function and interaction of every cell type in the boneforming/-resorbing assembly. Bone cells, unmistakably the most valuable asset for any bone regeneration strategy, are paradoxically the most difficult to exploit.

\section{The extracellular matrix of bone}

The extracellular matrix (ECM) holds the key to much of the naturally occurring regeneration of a tissue or organ, and as such, tissue engineers have long sought to utilize 
natural ECM or ECM-mimicking materials [82-84]. There are different compositions of ECM associated with different parts of bone, including the periosteum, osseous tissue and bone marrow. As osseous tissue best represents bone in term of structure and function, its ECM is often considered a good starting material for its rebuilding. Osseous ECM is an interstitial matrix consisting mainly of an organic matrix (collagens, non-collagenous proteins, proteoglycans and glycosaminoglycans) that bind tightly to hydroxyapatite (the mineral component). Whole osseous ECM, yet to be properly characterised, is made by processing bone tissue from allogeneic or xenogeneic sources (there is no reason to harvest and process autologous bone only for the ECM). Importantly, it is not that being derived from natural bone ECM makes for a good niche for bone cells. Indeed, no cell is able to squeeze through narrow canaliculi in order to re-inhabit the vacant bone lacunae. Therefore, any bone grafting material must be capable of being fully resorbed and replaced by new bone before the remodeling process completes [85]. Processing methods have been developed to make natural bone ECM more resorbable, including by physically breaking the tissue into smaller sizes (pulverization), chemically removing the mineral component (demineralization), or by partially enzymatically digesting the tissue (collagenase digestion) [86, 87]. Alternatively, one can synthesize materials utilizing one or more of the constituent bone ECM components, of which collagen, calcium phosphate and glycosaminoglycans are among the most often used.

Type I collagen is the most abundant organic component of bone ECM, though it is not specific to bone and is found in other tissues such as skin and tendon. Importantly, collagen is highly conserved in all vertebrates, and type I collagen from bovine and porcine sources has long been used in the clinic with good safety [88]. The role of collagen organization and its mineralization in the mechanical strength of bone is a complex subject and has been reviewed elsewhere [89]. As a bone substitute, however, collagen is the most extensively used biomaterial $[90,91]$. Physically, collagen materials can be made into injectable solutions, gels, malleable sponges, and solid forms of nearly any size and shape. Chemically, the collagen polypeptide possesses abundant functional groups, allowing modification such as crosslinking to other biomolecules such as glycosaminoglycans, RGD peptides, small molecules, and growth factors [92]. Overall, the collagen matrix induces haemostasis, improves biocompatibility of the synthetic scaffolds and promotes tissue engraftment, all of which are important for any healing process $[93,94]$. Due to its ubiquitous presence in many different tissues, collagen is unlikely to promote any specific healing trajectory, but rather to create a stabilized microenvironment where the cells and their growth factor interactions determine the next phase of the healing process [95]. For critical-sized bone defects, collagen matrix alone has been shown to improve healing, but it is insufficient for complete bone regeneration [96, 97]. On the other hand, the addition of BMP-2 on an absorbable collagen sponge can induce bone formation, even in ectopic locations.

The inorganic component of bone, the bone mineral (also termed bone salt or bone apatite), is a fascinating substance. Evolutionarily, as multicellular organisms became bigger, possession of a hardened internal structure offered tremendous advantages. Through millions of years, nature has come up with a myriad of ways to create biomaterials that are not only hard but also tough, light and dynamic. Mineralization of collagenous matrix is the process that blends the 
inorganic mineral salts into a living matrix of cells and proteins, forming the bone composite. The chemistry of bone mineral primarily involves modifications of the calcium phosphate, hydroxyapatite $\left(\mathrm{Ca}_{5}\left(\mathrm{PO}_{4}\right)_{3} \mathrm{OH}\right)$ [89, 99]. What is unique is the way these molecules are put together in a specific manner to form a rigid crystallized structure in tandem with the arrangement of collagen fibrils (Figure 3).

This mineral has been researched since at least the 1770s [100]. Much is now known about its micro- and nanostructure, as well as its potential use for bone regeneration [101]. Calcium phosphate materials have been shown to be not only biocompatible, but more importantly, they can be resorbed in the body without causing elevated calcium or phosphate levels in urine, serum, or other organs (liver, skin, brain, heart, kidney, lung, and intestine) [102]. Various types of calcium phosphate materials have been synthesized to differ in their chemistry or physical state, among them the group of ceramics, which have demonstrated superior performance for the support of bone regeneration [103].
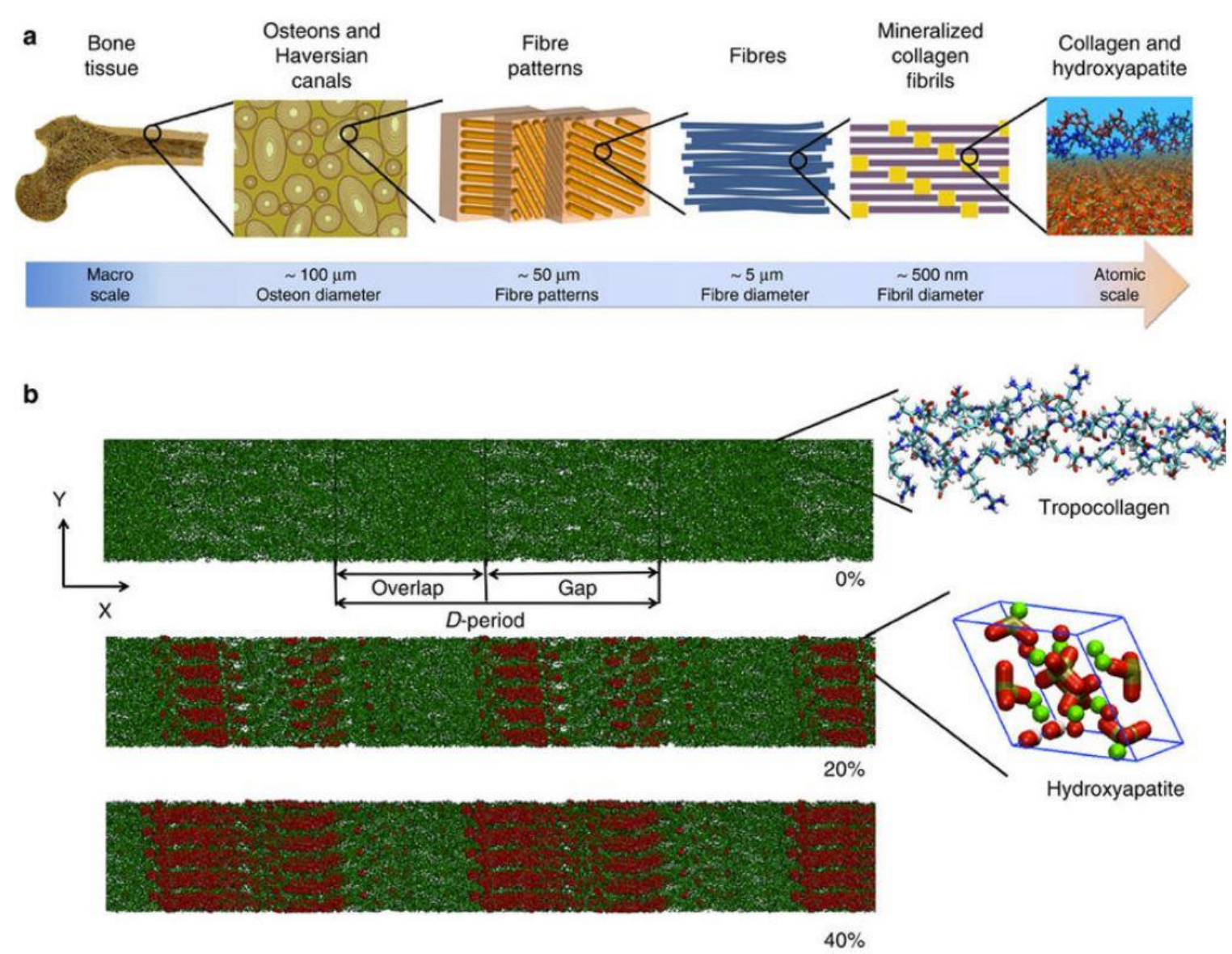

FIG. 3. Organization of collagen and hydroxyapatite in bone. Permission obtained from Springer Nature. (C) Nair, A.K. et al, Nat Commun. 4: p. 1724. (2013). [98] 
Apart from having good osteoconductivity - attracting the host bone to grow - calcium phosphate ceramics have even demonstrated osteoinductivity - the induction of bone formation outside the bone environment $[104,105]$. The osteoinductivity of calcium phosphate ceramics is significant, considering this ability involves both chemoattraction and differentiation of stem cells into bone-forming cells at the material site. Although it is not clear exactly how this works, the bone-inducing mechanism of such ceramics has started to be elucidated. Factors such as chemistry and topography are being isolated and systematically studied [106-109]. Undoubtedly, calcium phosphate materials have been and will be used extensively in many bone regeneration strategies.

Glycosaminoglycans (GAGs) are essential building blocks for life. GAGs are ubiquitously present in all tissues, in the ECM and on the surface of every cell. Unlike other biomolecules such as nucleic acids and proteins, GAGs are produced as a result of non-template-driven synthesis and thus are highly heterogeneous. Of the four different groups of GAGs (heparin/heparan sulfate, chondroitin sulfate (CS)/dermatan sulfate, keratin sulfate (KS) and hyaluronic acid), CS and KS are the most abundant in bone [110-113]. Other than serving a structural role, GAGs such as heparan sulfate (HS) are important modulators of cells and their interaction with growth factors $[114,115]$. The ability of HS to bind and protect growth factors from proteolytic degradation and even enhance the growth factor/receptor interactions has long been recognised. Many growth factors relevant to bone regeneration (such as FGFs, BMPs, TGFs, VEGFs, PDGFs) have been shown to bind to different species of HS through their unique heparin/HS-binding domains [116]. Unlike collagen, it is more difficult to isolate a large amount of HS from allogeneic or xenogeneic sources. The most convenient source is the byproduct of anticoagulant heparin isolation from animal organs such as porcine intestinal mucosa [117]. For this reason, different approaches to chemically engineer molecules with HS-like properties have been attempted.

One approach uses dextran derivatives (CMDBS), which mimic HS by protecting and stabilizing FGFs and have been shown to accelerate healing of cranial bones in a rodent model $[118,119]$. Indeed, a single dose of CMDBS delivered in a collagen sponge was shown to improve bone healing in critical-sized calvarial defects. As they later developed into a family of "regenerating agents", these HS-like polymers were able to stimulate tissue repair in many different tissues including bone, skin, muscle and cornea [120] by mimicking HS binding to endogenous growth factors (released during the healing process) in a nonspecific manner, so stabilizing them for prolonged action. Jackson et al. took a different approach by isolating bonederived HS from a culture of MC3T3-E1 preosteoblast cells that were used to augment fracture healing in rats [121]. The bone cell-derived HS enhanced growth factor activity within the callus, resulting in increased expression of osteoblastic genes. An even more specific approach was taken by Murali et al., using chromatography columns derivatized to capture HS species that have a high affinity for BMP-2 [122]. These affinity-isolated HS species enhanced overall BMP-2-induced osteogenesis by improving BMP-2 interaction with its receptor, thereby prolonging downstream signaling and, coincidentally, by also decoying the BMP-2 antagonist noggin. When the HS was combined with a collagen sponge to treat critical-sized bone defect in rabbits, the combination device was equally osteostimulatory to exogenous BMP-2. 
A recent development in GAG engineering has been the discovery of supramolecular selfassembled amphiphile glycopeptides, which display a high aspect ratio charge on their surface; even higher than heparin, the most negatively charged biomolecule in nature [123]. The glycopeptide amphiphile can bind strongly to many different growth factors, but, unlike heparin, it has almost no anticoagulant activity - the side effect that renders heparin inappropriate for tissue engineering applications. When combined with BMP-2 in a collagen sponge for a rat posterolateral spinal fusion model, the engineered sugar boosts the activity of a BMP-2 dose that is 100 times lower than the effective dose. GAGs, an often overlooked component of the bone ECM, should be more carefully considered in the design of future bone regeneration strategies.

\section{The future of bone tissue engineering}

The treatment of bone injury has been attempted as early as the beginning of medicine. Although not scientifically recorded, surgical interventions existed even in prehistorical times as suggested by paleopathological evidence such as set fractures, rickets and drilled skulls [124]. Materials such as animal bone, ivory, silver and gold have been used to replace missing teeth or bones. Later, as the result of the 19-20th-century technological revolution, the emergence of tools such as high-resolution microscopy, histological and staining techniques, Xray, computed tomography, and genomic and proteomic techniques, have boosted our understanding of bone biology enormously. Very large collaborative international research projects such as the human genome project, the human proteome project and the human protein atlas have been completed or are underway. Rather counterintuitively, this increasing tide of information has not created a proportionally clearer understanding of the interactions between the key biological systems of the human body. Every new signaling pathway adds more complexity and, unlike electronics, no one-input-one-output mechanism exists in biology. For scientists looking for evidence for the best method to regenerate bone, it becomes clear that there is no best method - the right approach will depend on every individual biological situation. Thus, gaining in-depth knowledge about how every system in the body functions and malfunctions is a must before we can implement more perfect, personalized medical treatment.

The second thing can be concluded from this recent influx of data is that any attempt to find a therapeutic solution using the usual try-and-error approach will be increasingly unlikely to work. The mere number of possible therapeutic combinations exceeds the capacity of any highthroughput-screening system. Fortunately, concomitant with the accumulation of "big data" is the rise of machine learning and other methods to replace or augment the human researcher. The important take-home message is: tissue engineering of bone (or any other living organs for that matter) is not as simplistic as combining a few cell types on some scaffolds with certain growth factors, followed by implantation in vivo hoping for complete restoration of the tissue. As it moves from the bench to the bedside, a much deeper and more comprehensive understanding of bone biology and medicine as a whole must be applied to customize the most suitable bone regenerating therapy for an individual patient. 


\section{Acknowledgments}

BL, VN, and SC acknowledge the financial support of A*STAR, Singapore. JdB, CvB and VL acknowledge the financial support of the Dutch province of Limburg.

\section{References for Chapter 2 (Review)}

1. Clarke, B., Normal bone anatomy and physiology. Clin J Am Soc Nephrol, 2008. 3 Suppl 3: p. S131-9.

2. Burt, L.A., D.A. Greene, and G.A. Naughton, Bone health of young male gymnasts: a systematic review. Pediatr Exerc Sci, 2017: p. 1-25.

3. Victoria, G., B. Petrisor, B. Drew, et al., Bone stimulation for fracture healing: What's all the fuss? Indian J Orthop, 2009. 43(2): p. 117-20.

4. Global, regional, and national incidence, prevalence, and years lived with disability for 301 acute and chronic diseases and injuries in 188 countries, 1990-2013: a systematic analysis for the Global Burden of Disease Study 2013. Lancet, 2015. 386(9995): p. 743-800.

5. Urist, M.R., Bone: formation by autoinduction. Science, 1965. 150(3698): p. 893-9.

6. Caplan, A.I., Mesenchymal stem cells. J Orthop Res, 1991. 9(5): p. 641-50.

7. Cranford, S.W., J. de Boer, C. van Blitterswijk, et al., Materiomics: an -omics approach to biomaterials research. Adv Mater, 2013. 25(6): p. 802-24.

8. Ollier, L., Recherches experimentales sur les greffes osseuses. 1860: impr. Jules Claye.

9. Duhamel, H., Sur le developpement et la crue des os des animaux. Mem Acad Roy Des Sci, 1742. 55: p. 354-357.

10. Guillot, D., Almost invisible, often ignored: periosteum, the living lace of bone. Medicographia, 2012. 34: p. 221-227.

11. OpenStar_College. "File:603-Anatomy of Long Bone"; "File:606-Spongy Bone"; "File:624Diagram of Compact Bone". [cited 2017 October 2017].

12. Doi, K. and K. Sakai, Vascularized periosteal bone graft from the supracondylar region of the femur. Microsurgery, 1994. 15(5): p. 305-15.

13. Vogelin, E., N.F. Jones, J.I. Huang, et al., Healing of a critical-sized defect in the rat femur with use of a vascularized periosteal flap, a biodegradable matrix, and bone morphogenetic protein. J Bone Joint Surg Am, 2005. 87(6): p. 1323-31.

14. Del Pinal, F., F.J. Garcia-Bernal, J. Regalado, et al., Vascularised corticoperiosteal grafts from the medial femoral condyle for difficult non-unions of the upper limb. J Hand Surg Eur Vol, 2007. 32(2): p. 135-42.

15. Fuchs, B., S.P. Steinmann, and A.T. Bishop, Free vascularized corticoperiosteal bone graft for the treatment of persistent nonunion of the clavicle. J Shoulder Elbow Surg, 2005. 14(3): p. 264-8.

16. Saito, M., H. Shimizu, M. Beppu, et al., The role of beta-tricalcium phosphate in vascularized periosteum. J Orthop Sci, 2000. 5(3): p. 275-82.

17. Owen, M., The origin of bone cells. Int Rev Cytol, 1970. 28: p. 213-38.

18. Nakahara, H., J.E. Dennis, S.P. Bruder, et al., In vitro differentiation of bone and hypertrophic cartilage from periosteal-derived cells. Exp Cell Res, 1991. 195(2): p. 492503.

19. Kisiel, A.H., L.A. McDuffee, E. Masaoud, et al., Isolation, characterization, and in vitro proliferation of canine mesenchymal stem cells derived from bone marrow, adipose tissue, muscle, and periosteum. Am J Vet Res, 2012. 73(8): p. 1305-17. 
20. Colnot, C., Skeletal cell fate decisions within periosteum and bone marrow during bone regeneration. J Bone Miner Res, 2009. 24(2): p. 274-82.

21. Bilkay, U., C. Tokat, E. Helvaci, et al., Osteogenic capacities of tibial and cranial periosteum: a biochemical and histologic study. J Craniofac Surg, 2008. 19(2): p. 453-8.

22. Uddstromer, L., The osteogenic capacity of tubular and membranous bone periosteum. A qualitative and quantitative experimental study in growing rabbits. Scand J Plast Reconstr Surg, 1978. 12(3): p. 195-205.

23. Schonmeyr, B., N. Clavin, T. Avraham, et al., Synthesis of a tissue-engineered periosteum with acellular dermal matrix and cultured mesenchymal stem cells. Tissue Eng Part A, 2009. 15(7): p. 1833-41.

24. Beniker, D., D. McQuillan, S. Livesey, et al., The use of acellular dermal matrix as a scaffold for periosteum replacement. Orthopedics, 2003. 26(5 Suppl): p. s591-6.

25. Hattori, K., T. Yoshikawa, Y. Takakura, et al., Bio-artificial periosteum for severe open fracture--an experimental study of osteogenic cell/collagen sponge composite as a bioartificial periosteum. Biomed Mater Eng, 2005. 15(3): p. 127-36.

26. Zhang, K.G., B.F. Zeng, and C.Q. Zhang, [Periosteum construction in vitro by small intestinal submucosa combined with bone marrow mesenchymal stem cell]. Zhonghua Wai Ke Za Zhi, 2005. 43(24): p. 1594-7.

27. Baldwin, J.G., F. Wagner, L.C. Martine, et al., Periosteum tissue engineering in an orthotopic in vivo platform. Biomaterials, 2017. 121: p. 193-204.

28. Aarden, E.M., E.H. Burger, and P.J. Nijweide, Function of osteocytes in bone. J Cell Biochem, 1994. 55(3): p. 287-99.

29. Sakaguchi, Y., I. Sekiya, K. Yagishita, et al., Suspended cells from trabecular bone by collagenase digestion become virtually identical to mesenchymal stem cells obtained from marrow aspirates. Blood, 2004. 104(9): p. 2728-35.

30. Tuli, R., M.R. Seghatoleslami, S. Tuli, et al., A simple, high-yield method for obtaining multipotential mesenchymal progenitor cells from trabecular bone. Mol Biotechnol, 2003. 23(1): p. 37-49.

31. Keaveny, T.M. and W.C. Hayes, Mechanical properties of cortical and trabecular bone. Bone, 1993. 7: p. 285-344.

32. Pape, H.C., A. Evans, and P. Kobbe, Autologous bone graft: properties and techniques. J Orthop Trauma, 2010. 24 Suppl 1: p. S36-40.

33. Burchardt, H., Biology of bone transplantation. Orthop Clin North Am, 1987. 18(2): p. 18796.

34. Khan, S.N., F.P. Cammisa, Jr., H.S. Sandhu, et al., The biology of bone grafting. J Am Acad Orthop Surg, 2005. 13(1): p. 77-86.

35. Minami, A., T. Kasashima, N. Iwasaki, et al., Vascularised fibular grafts. An experience of 102 patients. J Bone Joint Surg Br, 2000. 82(7): p. 1022-5.

36. Roberts, T.T. and A.J. Rosenbaum, Bone grafts, bone substitutes and orthobiologics: the bridge between basic science and clinical advancements in fracture healing. Organogenesis, 2012. 8(4): p. 114-24.

37. Raggatt, L.J. and N.C. Partridge, Cellular and molecular mechanisms of bone remodeling. J Biol Chem, 2010. 285(33): p. 25103-8.

38. Aldridge, J.M., 3rd and J.R. Urbaniak, Avascular necrosis of the femoral head: role of vascularized bone grafts. Orthop Clin North Am, 2007. 38(1): p. 13-22, v.

39. Hauschka, P.V., A.E. Mavrakos, M.D. Iafrati, et al., Growth factors in bone matrix. Isolation of multiple types by affinity chromatography on heparin-Sepharose. J Biol Chem, 1986. 261(27): p. 12665-74.

40. Blum, B., J. Moseley, L. Miller, et al., Measurement of bone morphogenetic proteins and other growth factors in demineralized bone matrix. Orthopedics, 2004. 27(1 Suppl): p. s1615 . 
41. Wildemann, B., A. Kadow-Romacker, N.P. Haas, et al., Quantification of various growth factors in different demineralized bone matrix preparations. J Biomed Mater Res A, 2007. 81(2): p. 437-42.

42. Drosos, G.I., P. Touzopoulos, A. Ververidis, et al., Use of demineralized bone matrix in the extremities. World J Orthop, 2015. 6(2): p. 269-77.

43. Zadegan, S.A., A. Abedi, S.B. Jazayeri, et al., Demineralized bone matrix in anterior cervical discectomy and fusion: a systematic review. Eur Spine J, 2017. 26(4): p. 958-974.

44. Gerard J. Tortora, B.H.D., The skeletal system: bone tissue, in Principles of anatomy and physiology. 2012, Wiley. p. 182-207.

45. McParland, B.J., Nuclear Medicine Radiation Dosimetry. 2010, Dordrecht: Springer.

46. Luk, S.C., C. Nopajaroonsri, and G.T. Simon, The ultrastructure of endosteum: a topographic study in young adult rabbits. J Ultrastruct Res, 1974. 46(2): p. 165-83.

47. Schepers, K., E.M. Pietras, D. Reynaud, et al., Myeloproliferative neoplasia remodels the endosteal bone marrow niche into a self-reinforcing leukemic niche. Cell Stem Cell, 2013. 13(3): p. 285-99.

48. Morrison, S.J. and D.T. Scadden, The bone marrow niche for haematopoietic stem cells. Nature, 2014. 505(7483): p. 327-34.

49. Bianco, P. and P.G. Robey, Skeletal stem cells. Development, 2015. 142(6): p. 1023-7.

50. Dominici, M., K. Le Blanc, I. Mueller, et al., Minimal criteria for defining multipotent mesenchymal stromal cells. The International Society for Cellular Therapy position statement. Cytotherapy, 2006. 8(4): p. 315-7.

51. Caplan, A.I. and D. Correa, The MSC: an injury drugstore. Cell Stem Cell, 2011. 9(1): p. $11-5$.

52. Tavassoli, M. and W.H. Crosby, Transplantation of marrow to extramedullary sites. Science, 1968. 161(3836): p. 54-6.

53. Friedenstein, A.J., S. Piatetzky, II, and K.V. Petrakova, Osteogenesis in transplants of bone marrow cells. J Embryol Exp Morphol, 1966. 16(3): p. 381-90.

54. Kuznetsov, S.A., P.H. Krebsbach, K. Satomura, et al., Single-colony derived strains of human marrow stromal fibroblasts form bone after transplantation in vivo. J Bone Miner Res, 1997. 12(9): p. 1335-47.

55. Gianakos, A., A. Ni, L. Zambrana, et al., Bone Marrow Aspirate Concentrate in Animal Long Bone Healing: An Analysis of Basic Science Evidence. J Orthop Trauma, 2016. 30(1): p. 1-9.

56. Gomez-Barrena, E., P. Rosset, D. Lozano, et al., Bone fracture healing: cell therapy in delayed unions and nonunions. Bone, 2015. 70: p. 93-101.

57. Le Nail, L.R., J. Stanovici, J. Fournier, et al., Percutaneous grafting with bone marrow autologous concentrate for open tibia fractures: analysis of forty three cases and literature review. Int Orthop, 2014. 38(9): p. 1845-53.

58. Bortolotti, F., L. Ukovich, V. Razban, et al., In vivo therapeutic potential of mesenchymal stromal cells depends on the source and the isolation procedure. Stem Cell Reports, 2015. 4(3): p. 332-9.

59. Corral, D.A., M. Amling, M. Priemel, et al., Dissociation between bone resorption and bone formation in osteopenic transgenic mice. Proc Natl Acad Sci U S A, 1998. 95(23): p. 1383540.

60. Long, F., Building strong bones: molecular regulation of the osteoblast lineage. Nat Rev Mol Cell Biol, 2011. 13(1): p. 27-38.

61. Knight, M.N. and K.D. Hankenson, Mesenchymal Stem Cells in Bone Regeneration. Adv Wound Care (New Rochelle), 2013. 2(6): p. 306-316.

62. da Silva Meirelles, L., A.I. Caplan, and N.B. Nardi, In search of the in vivo identity of mesenchymal stem cells. Stem Cells, 2008. 26(9): p. 2287-99. 
63. Murray, I.R. and B. Peault, $Q \& A$ : Mesenchymal stem cells - where do they come from and is it important? BMC Biol, 2015. 13: p. 99.

64. Uccelli, A., L. Moretta, and V. Pistoia, Mesenchymal stem cells in health and disease. Nat Rev Immunol, 2008. 8(9): p. 726-36.

65. Ullah, I., R.B. Subbarao, and G.J. Rho, Human mesenchymal stem cells - current trends and future prospective. Biosci Rep, 2015. 35(2).

66. Lalu, M.M., L. McIntyre, C. Pugliese, et al., Safety of cell therapy with mesenchymal stromal cells (SafeCell): a systematic review and meta-analysis of clinical trials. PLoS One, 2012. 7(10): p. e47559.

67. Goshima, J., V.M. Goldberg, and A.I. Caplan, The osteogenic potential of culture-expanded rat marrow mesenchymal cells assayed in vivo in calcium phosphate ceramic blocks. Clin Orthop Relat Res, 1991(262): p. 298-311.

68. Arinzeh, T.L., S.J. Peter, M.P. Archambault, et al., Allogeneic mesenchymal stem cells regenerate bone in a critical-sized canine segmental defect. J Bone Joint Surg Am, 2003. 85-A(10): p. 1927-35.

69. Kruyt, M.C., C.E. Wilson, J.D. de Bruijn, et al., The effect of cell-based bone tissue engineering in a goat transverse process model. Biomaterials, 2006. 27(29): p. 5099-106.

70. Siddappa, R., A. Martens, J. Doorn, et al., cAMP/PKA pathway activation in human mesenchymal stem cells in vitro results in robust bone formation in vivo. Proc Natl Acad Sci U S A, 2008. 105(20): p. 7281-6.

71. Chatterjea, A., G. Meijer, C. van Blitterswijk, et al., Clinical application of human mesenchymal stromal cells for bone tissue engineering. Stem Cells Int, 2010. 2010: p. 215625.

72. Rosset, P., F. Deschaseaux, and P. Layrolle, Cell therapy for bone repair. Orthop Traumatol Surg Res, 2014. 100(1 Suppl): p. S107-12.

73. Le Blanc, K., C. Gotherstrom, O. Ringden, et al., Fetal mesenchymal stem-cell engraftment in bone after in utero transplantation in a patient with severe osteogenesis imperfecta. Transplantation, 2005. 79(11): p. 1607-14.

74. Horwitz, E.M., P.L. Gordon, W.K. Koo, et al., Isolated allogeneic bone marrow-derived mesenchymal cells engraft and stimulate growth in children with osteogenesis imperfecta: Implications for cell therapy of bone. Proc Natl Acad Sci U S A, 2002. 99(13): p. 8932-7.

75. Miron, R.J. and D.D. Bosshardt, OsteoMacs: Key players around bone biomaterials. Biomaterials, 2016. 82: p. 1-19.

76. Batoon, L., S.M. Millard, L.J. Raggatt, et al., Osteomacs and Bone Regeneration. Curr Osteoporos Rep, 2017. 15(4): p. 385-395.

77. Wu, A.C., L.J. Raggatt, K.A. Alexander, et al., Unraveling macrophage contributions to bone repair. Bonekey Rep, 2013. 2: p. 373.

78. Burnett, S.H., E.J. Kershen, J. Zhang, et al., Conditional macrophage ablation in transgenic mice expressing a Fas-based suicide gene. J Leukoc Biol, 2004. 75(4): p. 612-23.

79. Alexander, K.A., M.K. Chang, E.R. Maylin, et al., Osteal macrophages promote in vivo intramembranous bone healing in a mouse tibial injury model. J Bone Miner Res, 2011. 26(7): p. 1517-32.

80. Davison, N.L., A.L. Gamblin, P. Layrolle, et al., Liposomal clodronate inhibition of osteoclastogenesis and osteoinduction by submicrostructured beta-tricalcium phosphate. Biomaterials, 2014. 35(19): p. 5088-97.

81. Chang, M.K., L.J. Raggatt, K.A. Alexander, et al., Osteal tissue macrophages are intercalated throughout human and mouse bone lining tissues and regulate osteoblast function in vitro and in vivo. J Immunol, 2008. 181(2): p. 1232-44.

82. Fernandes, H., L. Moroni, C. van Blitterswijk, et al., Extracellular matrix and tissue engineering applications. Journal of Materials Chemistry, 2009. 19(31): p. 5474-5484. 
83. Escobedo-Lucea, C., A. Ayuso-Sacido, C. Xiong, et al., Development of a human extracellular matrix for applications related with stem cells and tissue engineering. Stem Cell Rev, 2012. 8(1): p. 170-83.

84. Mouw, J.K., G. Ou, and V.M. Weaver, Extracellular matrix assembly: a multiscale deconstruction. Nat Rev Mol Cell Biol, 2014. 15(12): p. 771-85.

85. Hadjidakis, D.J. and Androulakis, II, Bone remodeling. Ann N Y Acad Sci, 2006. 1092: p. 385-96.

86. Eppley, B.L., W.S. Pietrzak, and M.W. Blanton, Allograft and alloplastic bone substitutes: a review of science and technology for the craniomaxillofacial surgeon. J Craniofac Surg, 2005. 16(6): p. 981-9.

87. Gruskin, E., B.A. Doll, F.W. Futrell, et al., Demineralized bone matrix in bone repair: history and use. Adv Drug Deliv Rev, 2012. 64(12): p. 1063-77.

88. Kontis, T.C. and A. Rivkin, The history of injectable facial fillers. Facial Plast Surg, 2009. 25(2): p. 67-72.

89. Viguet-Carrin, S., P. Garnero, and P.D. Delmas, The role of collagen in bone strength. Osteoporos Int, 2006. 17(3): p. 319-36.

90. Campana, V., G. Milano, E. Pagano, et al., Bone substitutes in orthopaedic surgery: from basic science to clinical practice. J Mater Sci Mater Med, 2014. 25(10): p. 2445-61.

91. Ferreira, A.M., P. Gentile, V. Chiono, et al., Collagen for bone tissue regeneration. Acta Biomater, 2012. 8(9): p. 3191-200.

92. Shoulders, M.D. and R.T. Raines, Collagen structure and stability. Annu Rev Biochem, 2009. 78: p. 929-58.

93. Gorgieva, S. and V. Kokol, Collagen-vs. gelatine-based biomaterials and their biocompatibility: review and perspectives, in Biomaterials applications for nanomedicine. 2011, InTech.

94. Chattopadhyay, S. and R.T. Raines, Review collagen-based biomaterials for wound healing. Biopolymers, 2014. 101(8): p. 821-33.

95. O'brien, F.J., Biomaterials \& scaffolds for tissue engineering. Materials today, 2011. 14(3): p. 88-95.

96. Sweeney, T.M., L.A. Opperman, J.A. Persing, et al., Repair of critical size rat calvarial defects using extracellular matrix protein gels. J Neurosurg, 1995. 83(4): p. 710-5.

97. Saadeh, P.B., R.K. Khosla, B.J. Mehrara, et al., Repair of a critical size defect in the rat mandible using allogenic type I collagen. J Craniofac Surg, 2001. 12(6): p. 573-9.

98. Nair, A.K., A. Gautieri, S.W. Chang, et al., Molecular mechanics of mineralized collagen fibrils in bone. Nat Commun, 2013. 4: p. 1724.

99. Glimcher, M.J., Bone: nature of the calcium phosphate crystals and cellular, structural, and physical chemical mechanisms in their formation. Reviews in mineralogy and geochemistry, 2006. 64(1): p. 223-282.

100. Dorozhkin, S., History of Calcium Phosphates in Regenerative Medicine, in Advances in Calcium Phosphate Biomaterials. 2014, Springer. p. 435-483.

101. Wang, P., L. Zhao, J. Liu, et al., Bone tissue engineering via nanostructured calcium phosphate biomaterials and stem cells. Bone Res, 2014. 2: p. 14017.

102. den Hollander, W., P. Patka, C.P. Klein, et al., Macroporous calcium phosphate ceramics for bone substitution: a tracer study on biodegradation with 45Ca tracer. Biomaterials, 1991. 12(6): p. 569-73.

103. Barrere, F., C.A. van Blitterswijk, and K. de Groot, Bone regeneration: molecular and cellular interactions with calcium phosphate ceramics. Int J Nanomedicine, 2006. 1(3): p. 317-32.

104. Yuan, H., K. Kurashina, J.D. de Bruijn, et al., A preliminary study on osteoinduction of two kinds of calcium phosphate ceramics. Biomaterials, 1999. 20(19): p. 1799-806. 
105. Cheng, L., T. Wang, J. Zhu, et al., Osteoinduction of Calcium Phosphate Ceramics in Four Kinds of Animals for 1 Year: Dog, Rabbit, Rat, and Mouse. Transplant Proc, 2016. 48(4): p. 1309-14.

106. Barradas, A.M., H.A. Fernandes, N. Groen, et al., A calcium-induced signaling cascade leading to osteogenic differentiation of human bone marrow-derived mesenchymal stromal cells. Biomaterials, 2012. 33(11): p. 3205-15.

107. Wennerberg, A. and T. Albrektsson, Effects of titanium surface topography on bone integration: a systematic review. Clin Oral Implants Res, 2009. 20 Suppl 4: p. 172-84.

108. Unadkat, H.V., M. Hulsman, K. Cornelissen, et al., An algorithm-based topographical biomaterials library to instruct cell fate. Proc Natl Acad Sci U S A, 2011. 108(40): p. 16565-70.

109. Groen, N., H. Yuan, D.G. Hebels, et al., Linking the Transcriptional Landscape of Bone Induction to Biomaterial Design Parameters. Adv Mater, 2017. 29(10).

110. Engfeldt, B. and A. Hjerpe, Glycosaminoglycans and proteoglycans of human bone tissue at different stages of mineralization. Acta Pathol Microbiol Scand A, 1976. 84(1): p. 95106.

111. Vejlens, L., Glycosaminoglycans of human bone tissue. I. Pattern of compact bone in relation to age. Calcif Tissue Res, 1971. 7(2): p. 175-90.

112. Vejlens, L., Glycosaminoglycans of human bone tissue. II. Chondroitin sulphate pattern of femoral cortex, medulla and epiphyseal plate in osteopetrosis. Calcif Tissue Res, 1972. 9(1): p. 54-66.

113. Coulson-Thomas, Y.M., V.J. Coulson-Thomas, A.L. Norton, et al., The identification of proteoglycans and glycosaminoglycans in archaeological human bones and teeth. PLoS One, 2015. 10(6): p. e0131105.

114. Perrimon, N. and M. Bernfield, Specificities of heparan sulphate proteoglycans in developmental processes. Nature, 2000. 404(6779): p. 725-8.

115. Bishop, J.R., M. Schuksz, and J.D. Esko, Heparan sulphate proteoglycans fine-tune mammalian physiology. Nature, 2007. 446(7139): p. 1030-7.

116. Hileman, R.E., J.R. Fromm, J.M. Weiler, et al., Glycosaminoglycan-protein interactions: definition of consensus sites in glycosaminoglycan binding proteins. Bioessays, 1998. 20(2): p. $156-67$.

117.Griffin, C.C., R.J. Linhardt, C.L. Van Gorp, et al., Isolation and characterization of heparan sulfate from crude porcine intestinal mucosal peptidoglycan heparin. Carbohydr Res, 1995. 276(1): p. 183-97.

118.Blanquaert, F., J.L. Saffar, M.L. Colombier, et al., Heparan-like molecules induce the repair of skull defects. Bone, 1995. 17(6): p. 499-506.

119.Albo, D., C. Long, N. Jhala, et al., Modulation of cranial bone healing with a heparin-like dextran derivative. J Craniofac Surg, 1996. 7(1): p. 19-22.

120.Barritault, D., M. Gilbert-Sirieix, K.L. Rice, et al., RGTA $(R)$ or ReGeneraTing Agents mimic heparan sulfate in regenerative medicine: from concept to curing patients. Glycoconj J, 2017. 34(3): p. 325-338.

121.Jackson, R.A., M.M. McDonald, V. Nurcombe, et al., The use of heparan sulfate to augment fracture repair in a rat fracture model. J Orthop Res, 2006. 24(4): p. 636-44.

122.Murali, S., B. Rai, C. Dombrowski, et al., Affinity-selected heparan sulfate for bone repair. Biomaterials, 2013. 34(22): p. 5594-605.

123.Lee, S.S., T. Fyrner, F. Chen, et al., Sulfated glycopeptide nanostructures for multipotent protein activation. Nat Nanotechnol, 2017. 12(8): p. 821-829.

124.Donati, D., C. Zolezzi, P. Tomba, et al., Bone grafting: historical and conceptual review, starting with an old manuscript by Vittorio Putti. Acta Orthop, 2007. 78(1): p. 19-25. 


\title{
Chapter 3
}

\section{High-Throughput Screening Assay for the Identification of Compounds Enhancing Collagenous Extracellular Matrix Production by ATDC5 Cells}

\author{
Bach Q. Le, Hugo Fernandes, Carlijn V.C. Bouten, Marcel Karperien, \\ Clemens van Blitterswijk, and Jan de Boer
}

$\mathrm{E}$ xtracellular matrix (ECM) provides not only structural support to the cells but also signals that regulate their fate. In many tissue engineering approaches, it remains challenging to achieve the right amount and type of ECM secreted by the cells to faithfully mimic the native tissue. In this article, we describe how to design and perform a highthroughput assay to screen for molecules capable of enhancing collagenous ECM (cECM) production. We chose ATDC5 cells to validate the assay since we want to use this chondrogenic cell line later for tissue engineering of hypertrophic cartilage. We used a fluorescently labeled collagen-binding probe to quantify total collagen content in ATDC5 cultures. The LOPAC1280 library of pharmaceutically active compounds was screened using insulin (a known inducer of cECM in ATDC5 cells) as positive control. After screening and validation, the small-molecule tetradecylthioacetic acid (TTA) was shown to enhance cECM production by ATDC5 cells at both gene expression and protein level. Moreover, when combined with insulin, TTA showed a synergistic effect on cECM production. In contrast, exposure of human primary chondrocytes and human mesenchymal stromal cells to TTA did not induce cECM secretion. In conclusion, we have developed an HTS assay to screen for compounds capable of enhancing cECM production and discovered TTA as a potent enhancer of cECM secretion by ATDC5 cells. 


\section{Introduction}

Many different cell types secrete an extracellular matrix (ECM), which provides mechanical support and serves as a signaling hub conveying signals from the cells to the surrounding environment and vice versa. Some of these signals control important biological functions such as cell viability and cell fate $[1,2]$. In tissue engineering, a common procedure is to isolate cells from their native tissue while leaving behind the ECM. The cells are then expanded to increase numbers, and are re-aggregated to mimic a three-dimensional (3D) environment, either by the use of a scaffold or simply by cell aggregation. Ideally, the cells in the tissue-engineered construct will adhere, proliferate, and secrete ECM. The nature of secreted ECM can be, to a certain extent, controlled by the signals provided by the scaffold (e.g., topographical cues) or by soluble signals to which the cells are exposed (e.g., growth factors or small molecules) [3-5].

In bone tissue engineering through endochondral ossification, the amount of hypertrophic cartilage matrix in the tissue-engineered construct is crucial for successful bone formation. We reported previously a method to efficiently generate bone in vivo using mouse embryonic stem cells pre-differentiated into hypertrophic chondrocytes in vitro [6]. The amount of bone detected in the construct after implantation was highly correlated with the amount of hypertrophic cartilage observed in vitro. Interestingly, the capacity of hypertrophic cartilage matrix to induce bone formation in vivo is not only limited to living cartilage. In 1958, Bridge and Pritchard performed a series of experiments, in which devitalized tissues were placed under the kidney capsules or implanted subcutaneously in the ears of rabbits. Tissues containing hypertrophic cartilage, which were devitalized with alcohol, acetone, and $\mathrm{HCl}$, or heated to $55^{\circ} \mathrm{C}$ consistently, formed bone [7].

Later, Marshall Urist also observed new bone formation when a boiled fracture callus was transplanted into the anterior chamber of a rat's eye [8-10]. This interesting phenomenon can be exploited to produce off-the-shelf bone-inducing hypertrophic cartilage since devitalized allografts provoke a much milder immune response compared with viable tissue $[11,12]$. We attempt to repeat the devitalized cartilage experiment, but instead of using natural hypertrophic tissue, such as growth plate cartilage or fracture callus, we try to tissue engineer hypertrophic cartilage in vitro to better control the state of hypertrophy. However, one final hurdle remains: production of sufficient amounts of ECM in vitro.

Previously, we used high-throughput screening (HTS) to search for small molecules that can modulate the cellular response of certain cell types. For instance, using HTS, we identified several compounds that improve osteogenic differentiation of the human mesenchymal stromal cell (hMSC) [13] and discovered that the small-molecule phenanthroline is very efficient in inducing angiogenesis [14]. In this article, we designed and performed an HTS assay to search for compounds that can enhance ECM production. We chose the chondrogenic cell line, ATDC5, for this HTS assay because it is a well-established cell line able to produce collagenous ECM (cECM) while growing in two dimensional (2D) culture on normal tissue culture-treated polystyrene surface, which simplifies the detection method. More importantly, ATDC5 cells can consistently differentiate into hypertrophic chondrocytes and produce hypertrophic cartilage matrix in vitro [15] which can be used later for our devitalized cartilage strategy. To quantify the amount of collagenous matrix produced by the cells, we use a 
fluorescently labeled collagen-binding probe, which has affinity for both fibrillar and nonfibrillar collagen $[16,17]$. This property, that is, not specific to any particular type of collagen, was exploited to measure the total amount of cECM produced by the cells. Using this assay, we identified and validated tetradecylthioacetic acid (TTA) as a compound capable of enhancing cECM production by ATDC5 cells.

\section{Materials and Methods}

\section{Cell culture}

The ATDC5 cell line was obtained from the RIKEN cell bank (Japan) and maintained in basic medium (BM), which consists of a 1:1 mixture of Dulbecco's modified Eagle's medium (DMEM) and Ham's F-12 supplied with Gluta-MAX ${ }^{\mathrm{TM}}$ (DMEM/F-12 GlutaMAX), 5\% v/v fetal bovine serum (FBS; Lonza), $0.2 \mathrm{mM}$ L-ascorbic acid-2 phosphate (ASAP), $100 \mathrm{U} / \mathrm{mL}$ of penicillin, and $100 \mathrm{mg} / \mathrm{mL}$ of streptomycin (PS). Cells were grown at $37^{\circ} \mathrm{C}$ in a humid atmosphere with $5 \% \mathrm{CO}_{2}$. The medium was refreshed twice a week and cells were used for further subculturing or cryopreservation upon reaching near confluence. To stimulate chondrogenesis and ECM production, BM was replaced with insulin medium (IM) consisting of $\mathrm{BM}$ supplemented with $1 \mathrm{x}$ insulin, transferrin, selenium solution (ITS) to yield a final concentration of $10 \mathrm{mg} / \mathrm{mL}$ insulin, $5.5 \mathrm{mg} / \mathrm{mL}$ transferrin, and $3.87 \times 10^{-8} \mathrm{M}$ sodium selenite [18].

Primary human mesenchymal stem cells (hMSCs, three donors) and human primary articular chondrocytes (hPCs, three donors) were obtained and isolated as described previously $[19,20]$. The use of bone marrow aspirates and human knee biopsies was approved by the Medical Ethics Committee of Medisch Spectrum Twente, and informed consent was obtained from all patients. hMSCs were expanded at the initial seeding density of $1000 \mathrm{cells} / \mathrm{cm}^{2}$ in proliferation medium consisting of $\alpha$-MEM, 10\% v/v FBS (Lonza), $2 \mathrm{mM}$ L-glutamine, $0.2 \mathrm{mM}$ ASAP, PS, and $1 \mathrm{ng} / \mathrm{mL}$ recombinant human basic fibroblast growth factor (AbD Serotec).

For chondrogenic differentiation, hMSCs (passage 2) were seeded at $2.5 \times 10^{5}$ cells per well in Cellstar U-shaped 96-well plates (Greiner Bio-one) and centrifuged for $3 \mathrm{~min}$ at $300 \mathrm{~g}$ to form pellets. Chondrogenic medium for hMSCs consists of DMEM high glucose, $100 \mathrm{mg} / \mathrm{mL}$ sodium pyruvate, $0.2 \mathrm{mM}$ ASAP, PS, 1x ITS, $0.1 \mathrm{mM}$ dexamethasone (Dex), and $10 \mathrm{ng} / \mathrm{mL}$ transforming growth factor 3 (TGF 33 ; R\&D systems, 243-B3- 010). hMSC pellets were cultured for 28 days, and the medium was changed two to three times per week. hPCs were expanded in chondrocyte proliferation medium consisting of DMEM, 10\% v/v FBS, 1x nonessential amino acid, $0.2 \mathrm{mM}$ ASAP, $0.4 \mathrm{mM}$ proline, and PS.

For chondrogenic differentiation, hPCs (passage 1) were seeded at $10,000 \mathrm{cells} / \mathrm{cm}^{2}$ in sixwell plates. Chondrogenic medium for hPCs was the same as for hMSCs with the addition of 2 $\mathrm{mM}$ L-glutamine. hPCs were cultured for 14 days, and the medium was changed two to three times per week. All media and medium supplements were purchased from Life Technologies; all common chemicals were purchased from Sigma-Aldrich, unless otherwise stated. 


\section{Collagen probe CN35-AF488 assay}

Cells were cultured on a 96-well black/clear imaging plate (BD Bioscience; 353219). Per time point, the culture medium was replaced with staining medium, which consists of $0.25 \mathrm{mM}$ CN35-AF488 diluted in DMEM/F12 without supplements. CN35-AF488 consists of a collagenbinding protein domain (CNA35) conjugated to the fluorescent dye, Alexa Fluor 488 [16, 17]. Using solid-phase binding assays and immunohistological staining, Krahn et al. showed that the collagen probe bound relatively well to all types of collagen that were tested with a different affinity for the different collagen types (I-VI), but did not bind to any of the other ECM proteins [16]. The plates were incubated at $37^{\circ} \mathrm{C}$ in a humid atmosphere with $5 \% \mathrm{CO}_{2}$ on an orbital shaker $(20 \mathrm{rpm}$ ) for $1 \mathrm{~h}$. After that, the plates were washed thrice with PBS (Gibco) to remove the unbound probe. Then, total fluorescent intensity of CNA35-AF488 was measured at $520 \mathrm{~nm}$ on a Victor plate reader (PerkinElmer).

\section{High-throughput screen}

ATDC5 cells were seeded at 5000 cells $/ \mathrm{cm}^{2}$ in a 96-well black/clear imaging plate (BD Bioscience; 353219) in BM and allowed to proliferate for 3 days. Then, the 1280 test compounds (LOPAC1280; Sigma Aldrich) were added at a final concentration of $4.5 \mathrm{mM}$ in BM. BM and IM were used as negative (NC) and positive (PC) control, respectively. DMSO, the solvent used in the library at $0.25 \% \mathrm{v} / \mathrm{v}$, was added to the controls. The medium, including compounds, was changed after 3 days, and after 6 days, the collagen probe CNA35- AF488 assay was performed. A compound was defined as a hit if its signal was greater than the mean plus three times standard deviation of the negative controls in the same plate.

\section{Hits validation}

Three different concentrations of each hit compound were tested for their capacity to enhance ECM production. The experimental protocol was the same as the primary screen, with the exception that each compound and concentration was tested in triplicate. A PrestoBlue assay was performed to assess the effect of the compound on metabolic activity, representing cell viability. Briefly, BM containing 10\% (v/v) PrestoBlue solution (Invitrogen) was added and incubated at $37^{\circ} \mathrm{C}$ for $1 \mathrm{~h}$. Then, fluorescence was measured at $590 \mathrm{~nm}$ on a Victor plate reader (PerkinElmer). The concentration that induced the highest secretion of cECM without affecting the metabolic activity was selected for subsequent experiments.

\section{Gene expression analysis}

ATDC5 cells $\left(5000\right.$ cells $\left./ \mathrm{cm}^{2}\right)$ and hPCs $\left(10,000\right.$ cells $\left./ \mathrm{cm}^{2}\right)$ were seeded in triplicate in sixwell plates in BM and allowed to attach overnight. hMSCs $\left(2.5 \times 10^{5}\right.$ cells per well) were aggregated in U-shaped 96-well plates in hMSC chondrogenic differentiation medium and allowed to form pellets overnight. Then, the compounds were added, and the medium was changed two to three times per week. At selected time points, RNA was isolated using an RNA II nucleospin RNA isolation kit (Machery Nagel). For chondrogenic differentiation of hMSCs, three pellets were pooled for RNA isolation. RNA concentrations were measured using an ND100 spectrophotometer (Nanodrop1000). cDNA was synthesized from $1 \mathrm{mg}$ of RNA using iScript (BioRad) according to the manufacturer's protocol. qPCR was performed using $50 \mathrm{ng}$ of cDNA, $0.4 \mathrm{mM}$ of each forward and reverse primer (Sigma Genosys), and 1x SensiMix SYBR 
and Fluorescein master mix (Bioline). Primer sequences are shown in Supp Table 2. Real-time qPCR was performed in a Biorad My IQ5 machine (Biorad). Data were analyzed using the fit point method of My IQ5 software. The baseline was calculated automatically by the software at the lower log linear part above baseline noise, and the crossing temperature (Ct value) was determined. $\mathrm{Ct}$ values were normalized to the Beta-2 microglobulin (B2M) housekeeping gene, and $\mathrm{DCt}(\mathrm{Ct}$, control-Ct, sample) was used to calculate the upregulation in gene expression.

\section{Total glycosaminoglycan quantification assay}

Glycosaminoglycan (GAG) assay was performed as described previously [20]. Briefly, samples were washed with PBS and frozen at $-80^{\circ} \mathrm{C}$. Subsequently, they were digested with 1 $\mathrm{mg} / \mathrm{mL}$ proteinase $\mathrm{K}$ (Sigma-Aldrich) in Tris/EDTA buffer ( $\mathrm{pH} 7.6$ ) containing $18.5 \mathrm{mg} / \mathrm{mL}$ iodoacetamide and $1 \mathrm{mg} / \mathrm{mL}$ pepstatin A (Sigma-Aldrich) overnight at $56^{\circ} \mathrm{C}$. GAG content was spectrophotometrically determined with 9-dimethylmethylene blue chloride (DMMB; SigmaAldrich) staining in PBE buffer (14.2 g/L Na $2 \mathrm{HPO}_{4}$ and $3.72 \mathrm{~g} / \mathrm{L} \mathrm{Na}$ EDTA at pH 6.5) with a microplate reader (Bio-TEK instruments) at $520 \mathrm{~nm}$. The standard curve for the GAG analysis was generated using chondroitin sulfate A (from bovine trachea; Sigma-Aldrich).

\section{Hydroxyproline assay}

A hydroxyproline assay kit was purchased from Biovision (K555-100). The assay was performed according to the manufacturer's protocol. Briefly, samples (cell layer) in triplicate were retrieved from tissue culture flasks at weeks $1,2,3$, and 4 using cell scrapers and transferred to pressure tight Teflon-capped vials for hydrolysis in $6 \mathrm{M} \mathrm{HCl}$ for $3 \mathrm{~h}$ at $120^{\circ} \mathrm{C}$. Lysates were transferred to a 96-well plate and dried under vacuum. Chloramine $\mathrm{T}$ and 4 (Dimethylamino)benzaldehyde (DMAB) reagent were added to each sample and incubated for $90 \mathrm{~min}$ at $60^{\circ} \mathrm{C}$. Absorbance at $560 \mathrm{~nm}$ was measured in a microplate reader (Bio-TEK instruments). The standard curve was generated using 4-hydroxyproline as indicated in the manufacturer's protocol.

\section{$\underline{\text { Statistics }}$}

One-way or two-way ANOVA with Bonferroni's posttest and Pearson correlation analysis were performed using GraphPad Prism version 6.02 for Windows, GraphPad Software.

\section{Results}

\section{ECM production in differentiating ATDC5 cells}

ATDC5 cells produce high amounts of cECM in 2D culture. To characterize our ATDC5 cell line, we cultured the cells for 3-4 weeks in BM and IM and analyzed their morphology and the expression profile of cECM-related genes. Figure 1A shows the morphological changes of the ATDC5 cell layer upon exposure to IM over 3 weeks. At day 7, the cell layer morphology was similar for both conditions. At day 14, the ATDC5 cell layer treated with insulin developed many aggregates, which stain positive for Alcian Blue (data not shown), and further developed into nodules after 21 days. In contrast, in BM, small aggregates appeared at day 21, 1 week later than in IM. However, when cultured for up to 40 days, these aggregates did not progress into nodules as seen in the IM (data not shown). The ATDC5 nodules did not stain positive for 
Alizarin Red (data not shown) unless the medium was switched to mineralization medium supplemented with calcium and phosphate salts.

Gene expression analysis showed an increase in mRNA levels for collagen type II and type $\mathrm{X}$ in BM and IM over a 4-week time course (Fig. 1B). After 1 week, IM led to a 5-fold increase in collagen type II expression compared with BM, which increased to a 13-fold change upon 2 weeks and decreased thereafter to its basal level. A similar profile was observed for collagen type $\mathrm{X}$ expression (although in this case the fold change between the conditions reached a maximum of 60 at 2 weeks). A delay in the expression of both genes was observed in BM with maximum expression observed after 4 weeks instead of two for IM.

A

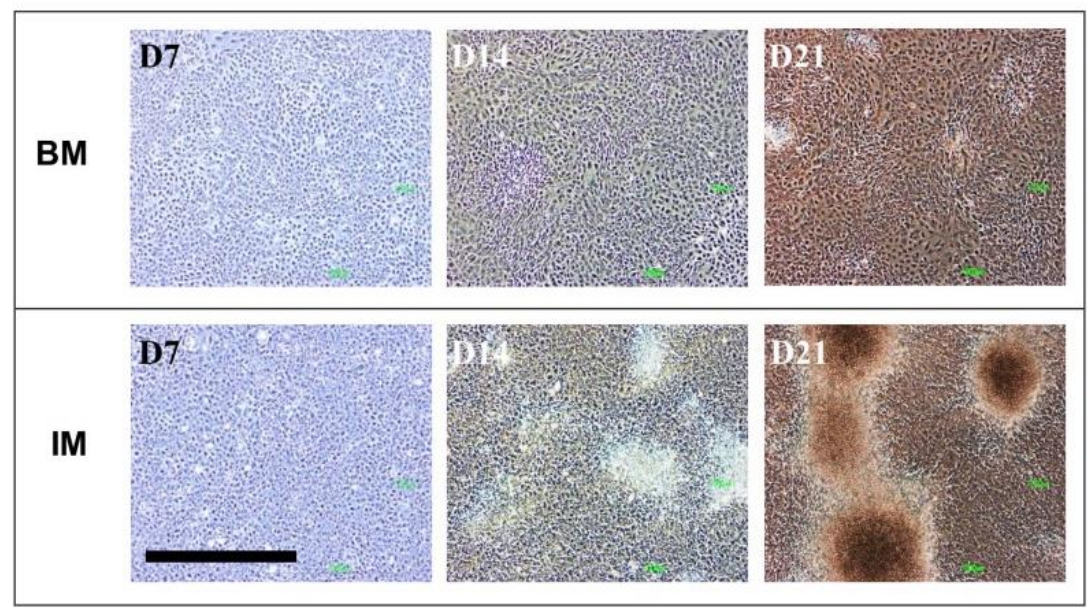

\section{B}
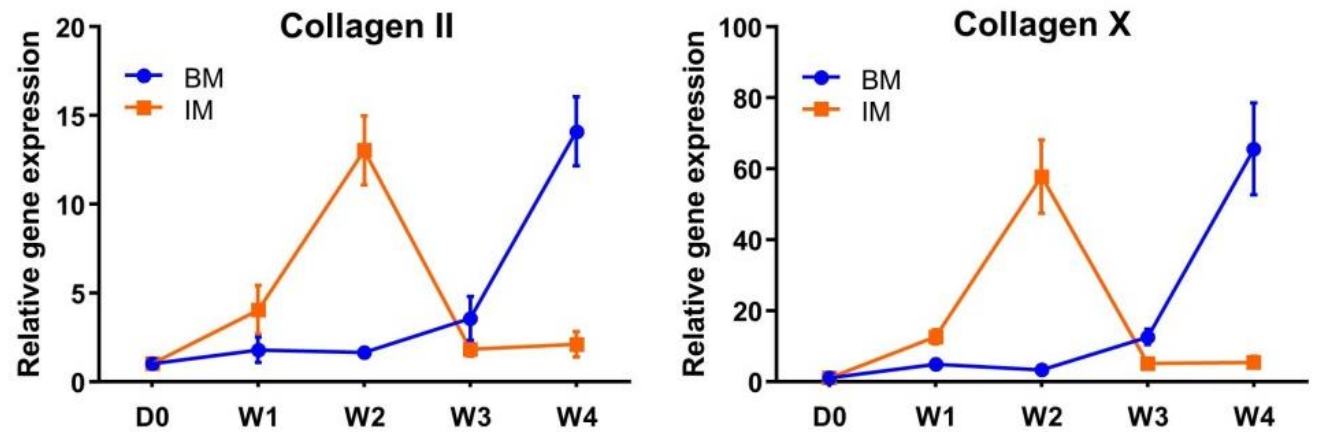

FIG. 1. Characterization of ATDC5 cell line. (A) Representative microscopy pictures of ATDC5 cultured on treated polystyrene surface (TCP) at days (D) 7, 14, and 21 in basic medium (BM) (top panel) or insulin medium (IM) (bottom panel). Scale bar represents $1 \mathrm{~mm}$. (B) Gene expression profile of collagen type II and X in ATDC5 cultured on TCP in BM (blue line) or IM (red line) at D0, weeks (W) 1, 2, 3, and 4. $(\mathrm{n}=3)$.

\section{Quantification of total collagen}


To quantify the total collagen content, we stained the cells with the CNA35-AF488 collagen probe. Figure 2A shows fluorescent images of ATDC5 cells, grown in BM and IM for 10 days, stained with the collagen probe. While ATDC5 cells also produce a basal level of collagen in the BM, the collagen network becomes much denser in the IM. The time point day 10 was chosen in our preliminary experiments; after this time, the cell layer in IM was already too thick and could detach easily in the staining and washing steps, affecting the fluorescent quantification. To demonstrate the capacity of the collagen probe assay, we compared it with a common GAG assay in a dose-response experiment of insulin on ATDC5 cells. Total fluorescence intensity of the CNA35-AF488 using the collagen probe assay (Fig. 2B) was highly correlated with increasing insulin concentration $\left(\mathrm{R}^{2}=0.8, \mathrm{p}=0.0066\right)$. Using the GAG assay (Fig. 2C), total GAG content and insulin concentration were less correlated $\left(\mathrm{R}^{2}=0.37, \mathrm{p}\right.$ $=0.1442$ ). Thus, a collagen probe assay has better resolution in measuring the response of ATDC5 cells to changes in stimuli.

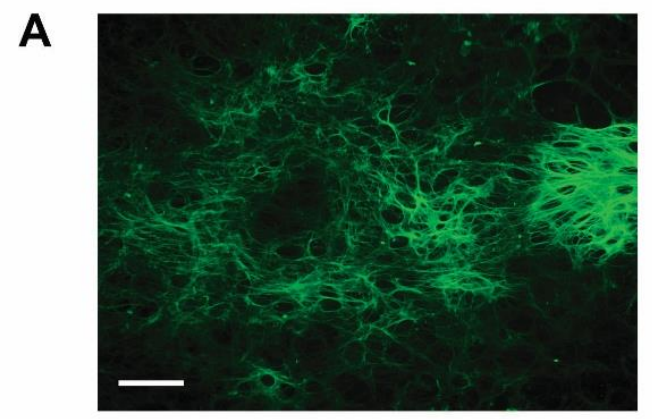

BM

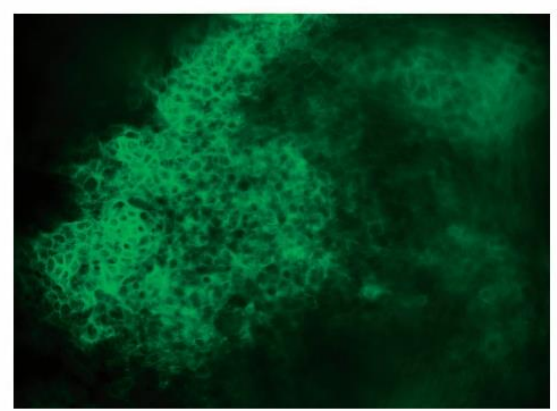

IM
B

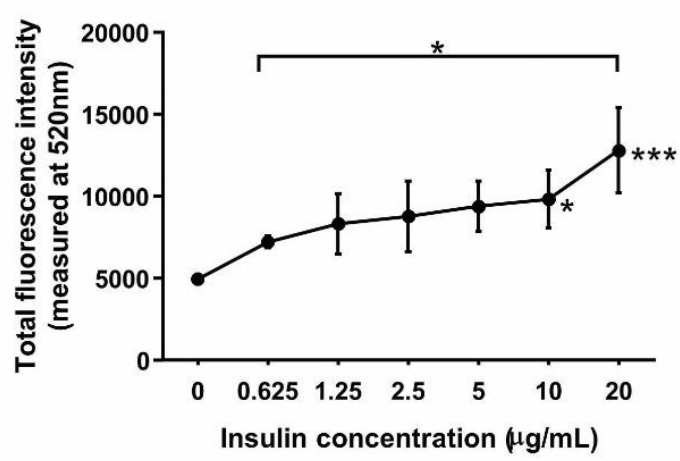

C

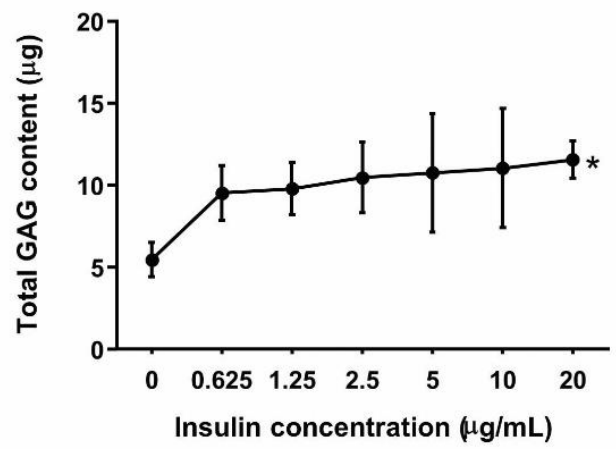

FIG. 2. Sensitivity of collagen probe CNA35-Alexa488 to detect extracellular matrix (ECM). (A) Fluorescence images of ATDC5 cultured on TCP for 10 days in BM or IM, stained with collagen probe CNA35-Alexa488, and visualized using a BD pathway 432 microscope. Scale bar represents $100 \mu \mathrm{m}$. Dose response of ATDC5 to insulin after 10 days cultured on TCP, (B) stained with collagen probe CNA35-Alexa488, and total fluorescence intensity measured by a Victor plate reader, or (C) quantified by GAG assay. Error bars represent standard deviation $(n=3)$. Statistical analysis was performed using oneway ANOVA and Bonferroni's post-test. (*) denotes $\mathrm{p}<0.05$ compared with basic $(0 \mathrm{mg} / \mathrm{mL}$ insulin) and $(* * *)$ denotes $\mathrm{p}<0.001$ compared with basic. 


\section{$\underline{\text { Screening for compounds enhancing ECM production by ATDC5 cells }}$}

To find small molecules capable of enhancing cECM production by ATDC5 cells, we screened the LOPAC1280 library (containing 1280 different compounds) using total fluorescence intensity of the collagen probe CNA35-AF488 staining as readout (Supp Fig. 1A). Supp Fig. 1B shows the mean and standard deviation of the PC and NC in every plate. Total cECM content was significantly increased $(\mathrm{p}<0.0001)$ when insulin was added $(1.5$-fold increase in total fluorescence intensity: $\mathrm{NC}=15264 \pm 1552$ vs. $\mathrm{PC}=22,718 \pm 1294)$. Since insulin is a very potent cECM inducer for ATDC5 cells, no compound was able to induce cECM secretion to a higher level than that of the IM in this screen. Thus, we decided to define hits based on their ability to enhance cECM production to a significantly higher level than BM, for which a hit selection threshold was chosen as the mean plus three times the standard deviation of the negative controls in the same plate.

Based on this criterion, we identified in total 13 hit compounds (Supp. Table 1), 6 of which were ordered for further testing. Selected hits were further tested using a wider range of concentrations (2-200 mM) to confirm the cECM-inducing effect on ATDC5 cells. As in the HTS assay, BM and IM were used as NC and PC, respectively. Metabolic activity assay was performed to examine potential cytotoxic effects of the compounds. Of the six compounds tested, only DL-Cycloserine and tetradecylthioacetic acid (TTA) were able to reproduce the cECM-inducing effect found on the HTS assay, inducing a significantly higher amount of cECM compared with the NC (Figs. 3B-C).

\section{Expression of ECM genes}

Next, we investigated the effect of the selected compounds on ATDC5 cells at the mRNA level. There are many different cECM proteins, but we were mostly interested in collagen type II and aggrecan since these cECM proteins form the major structural component of cartilage, especially articular cartilage. ATDC5 cells were cultured in the presence of the selected compounds for 10 days. The concentrations tested were the ones that induced the highest amount of CECM in the previous validation experiment. IM was again used as a positive control and compared with BM, it upregulated collagen type II expression 2.5 times and aggrecan 9 times. From the tested compounds, only $20 \mathrm{mM}$ TTA was able to induce a significantly higher expression of collagen type II (2-fold) and aggrecan (2.6-fold) compared with the BM (Figs. 4A-B).

Based on this result and the results from the previous experiment, we decided to select TTA for further testing. To further explore the effect of TTA on ATDC5 cells, we tested TTA in a narrower range of concentrations spanning the $20 \mathrm{mM}$ previously tested (from $10 \mathrm{mM}$ to $80 \mathrm{mM})$. Furthermore, we exposed cells to both TTA and insulin to see whether they could have a synergistic or additive effect on $\mathrm{cECM}$ production by ATDC5 cells. Interestingly, the expression of collagen type II (Fig. 4C) and aggrecan (Fig. 4D) was significantly higher when TTA was added to IM. At the protein level (using the collagen probe assay), $10 \mathrm{mM}$ TTA combined with IM induced a significantly higher amount of ECM compared with IM alone (Fig. 4E). 

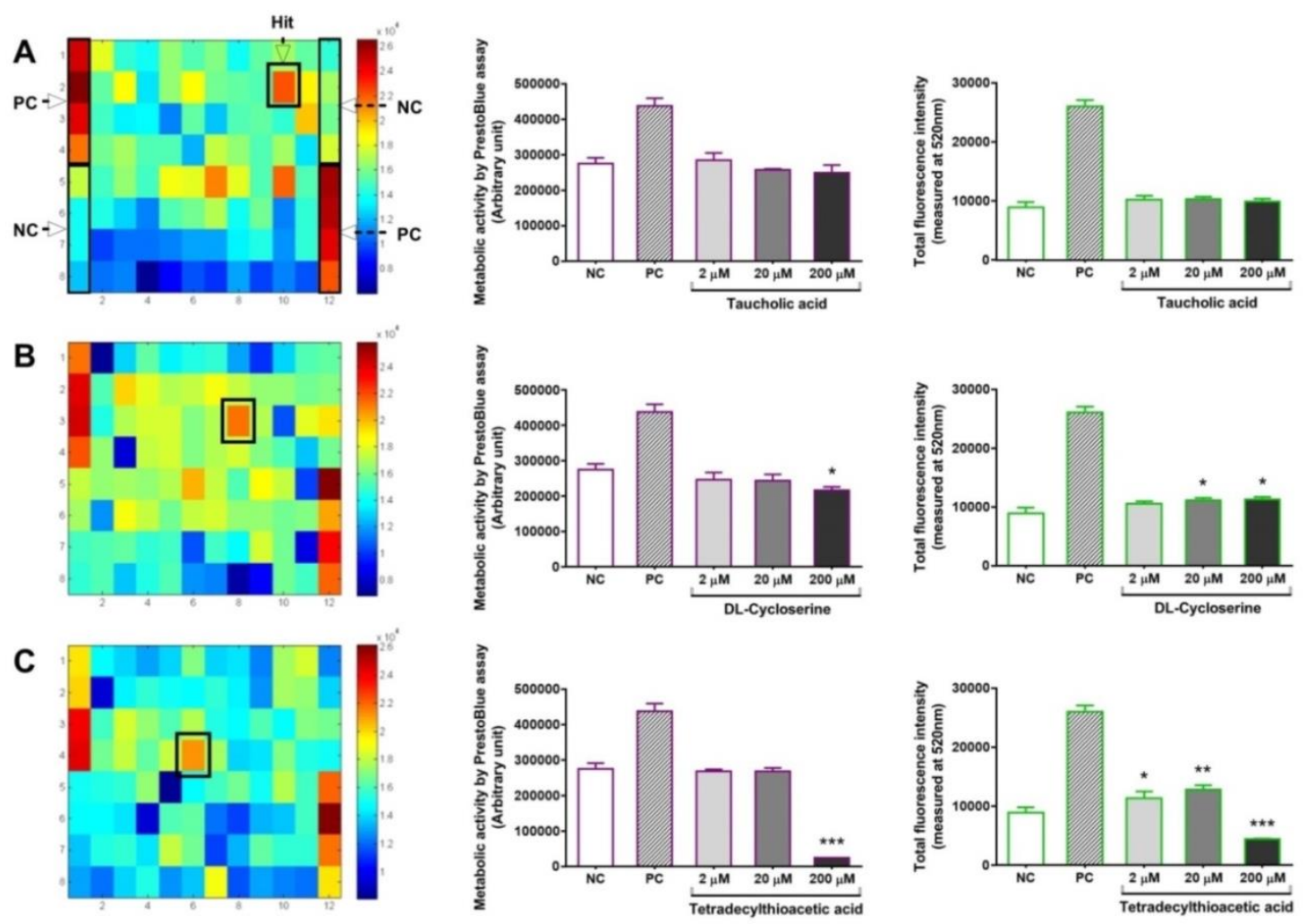

FIG. 3. Hit identification and validation. Representative Figures show how hits were identified and validated for (A)-plate 1 (identify taucholic acid), (B)-plate 4 (identify DLCycloserine), and (C)-plate 15 (identify tetradecylthioacetic acid). Plate number is specified in the LOPAC1280 catalog. Heat maps (left) show relative total fluorescence intensity of each well on the plate compared with the PC and NC. Bar graphs (middle) show the effects of different concentrations of hit compounds on metabolic activity of ATDC5. Bar graphs (right) validate the capability of hit compounds in different contractions to enhance collagenous ECM production of ATDC5, quantified by the collagen probe assay. Error bars represent standard deviation $(n=3)$. Statistical analysis was performed using one-way ANOVA and Bonferroni's post-test. $\left(^{*}\right)$ denotes $\mathrm{p}<0.05$ compared with $\mathrm{NC},(* *)$ denotes $\mathrm{p}<0.01$ compared with $\mathrm{NC}$, and $(* * *)$ denotes $\mathrm{p}<0.001$ compared with NC.

\section{Effect of TTA in long-term culture with ATDC5}

To test the effect of TTA on ATDC5 cells in long-term culture, we cultured ATDC5 cells in BM and IM with or without $20 \mathrm{mM}$ TTA for 4 weeks. Figure 5 shows representative pictures of the cell layers in different conditions and different time points. Over time, the cell layers in all conditions developed cell aggregations, which grew into nodules at later time points. In the presence of $20 \mathrm{mM}$ TTA, this process was accelerated. Moreover, with TTA, the nodules were often bigger and denser. 
A

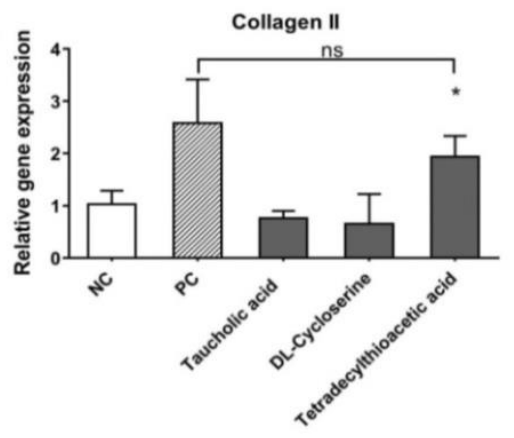

B

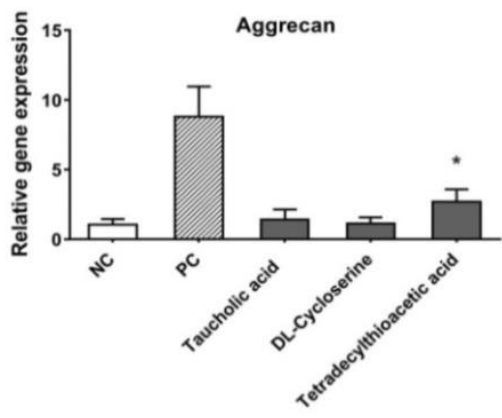

C

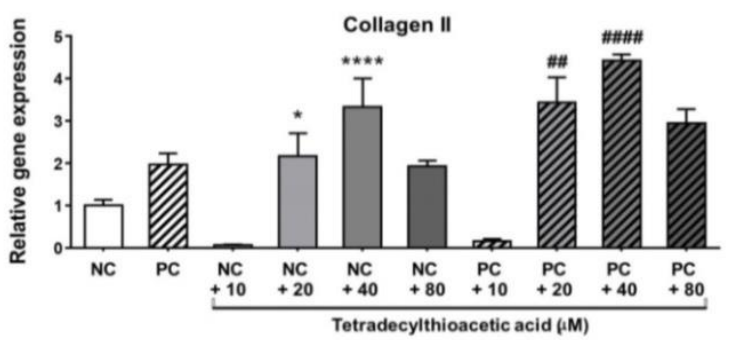

D

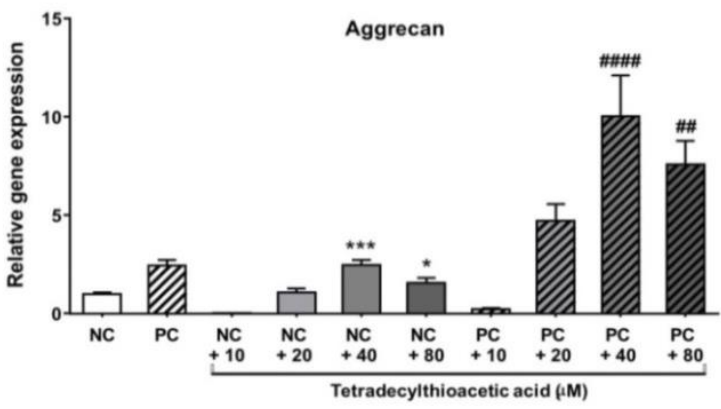

E

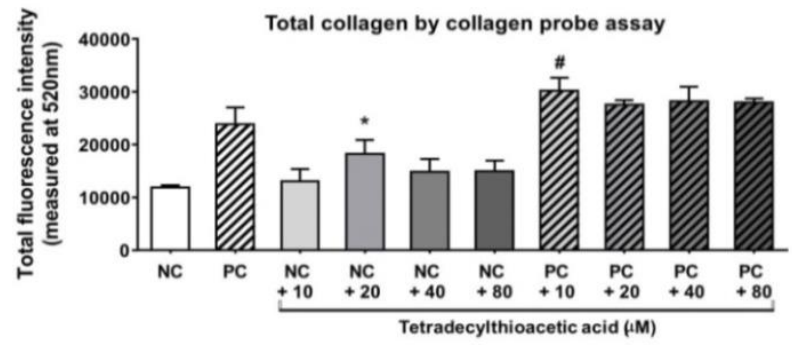

FIG. 4. Further validation of hit compounds by gene expression analysis. Collagen type II expression (A) and aggrecan expression (B) of ATDC5 treated with the hit compounds for 10 days. The concentration of the compounds was determined from the previous validation experiments. Only tetradecylthioacetic acid (TTA) showed a significant difference compared with the NC and thus was further tested in a dose-response experiment. (C-E) Dose-response effect of TTA on ATDC5. TTA was added at different concentrations to BM and IM. Collagen II expression (C), aggrecan expression (D), and total collagen quantification by collagen probe assay (E) were measured after 10 days. Notably, when combined with IM, TTA exerted a synergistic effect on aggrecan expression of ATDC5. Error bars represent standard deviation $(n=3)$. Statistical analysis was performed using one-way ANOVA and Bonferroni's post-test. (*) denotes a significant difference compared with the BM and (\#) denotes a significant difference compared with the IM. $(*)$ or $(\#)$ denotes $\mathrm{p}<0.05,(* *)$ or $(\# \#)$ denotes $\mathrm{p}<0.01,(* * *)$ denotes $\mathrm{p}<0.001$, and $(* * * *)$ or (\#\#\#) denotes $\mathrm{p}<0.0001 . \# \#$, ns, non-significant. 
Gene expression analysis was performed for collagen type II, collagen type X, aggrecan, and Sox9 (Figs. 6A-D). In the presence of $20 \mathrm{mM}$ TTA, expression of all these genes was upregulated compared with the conditions without TTA (except for Sox 9 at week 3 and 4). The upregulation was more pronounced between weeks 2 and 4 . A hydroxyproline assay was also performed to estimate total collagen content of the ECM derived from ATDC5 cells (Fig. 6E). Although hydroxyproline content of all conditions increased consistently over time, we did not find any statistically significant difference between the TTA conditions and other conditions mostly due to the large technical variation in the different replicates. Even the difference in the amount of hydroxyproline in IM versus BM was not evident until week 4 when a statistically significant difference was observed.

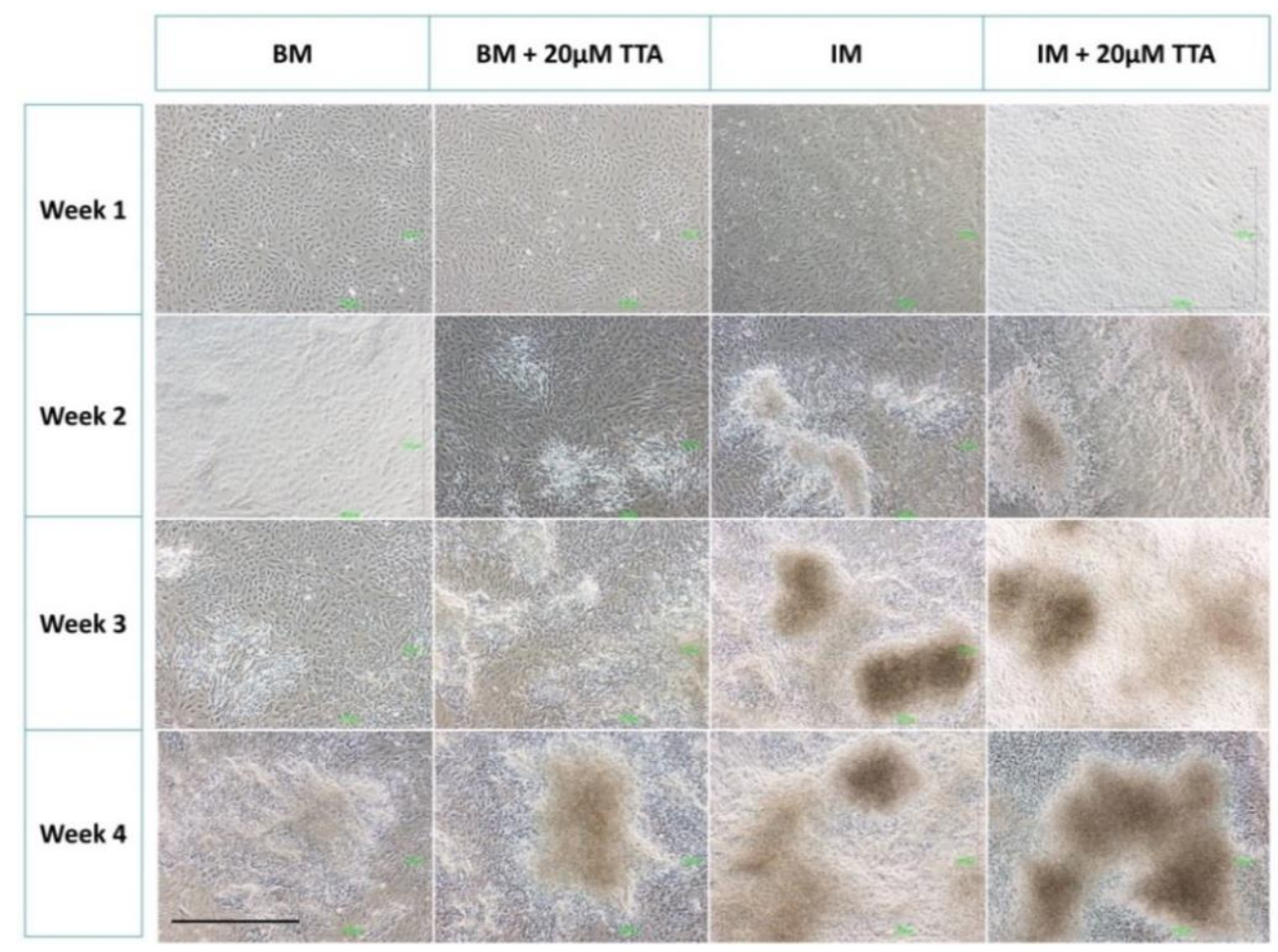

FIG. 5. ATDC5 cell layer in long-term culture with TTA. Representative microscopy pictures of ATDC5 cultured on TCP at weeks $1-4$ in BM, BM+ $20 \mathrm{mM}$ TTA, IM, and IM+ $20 \mathrm{mM}$ TTA. Scale bar represents $1 \mathrm{~mm}$. 

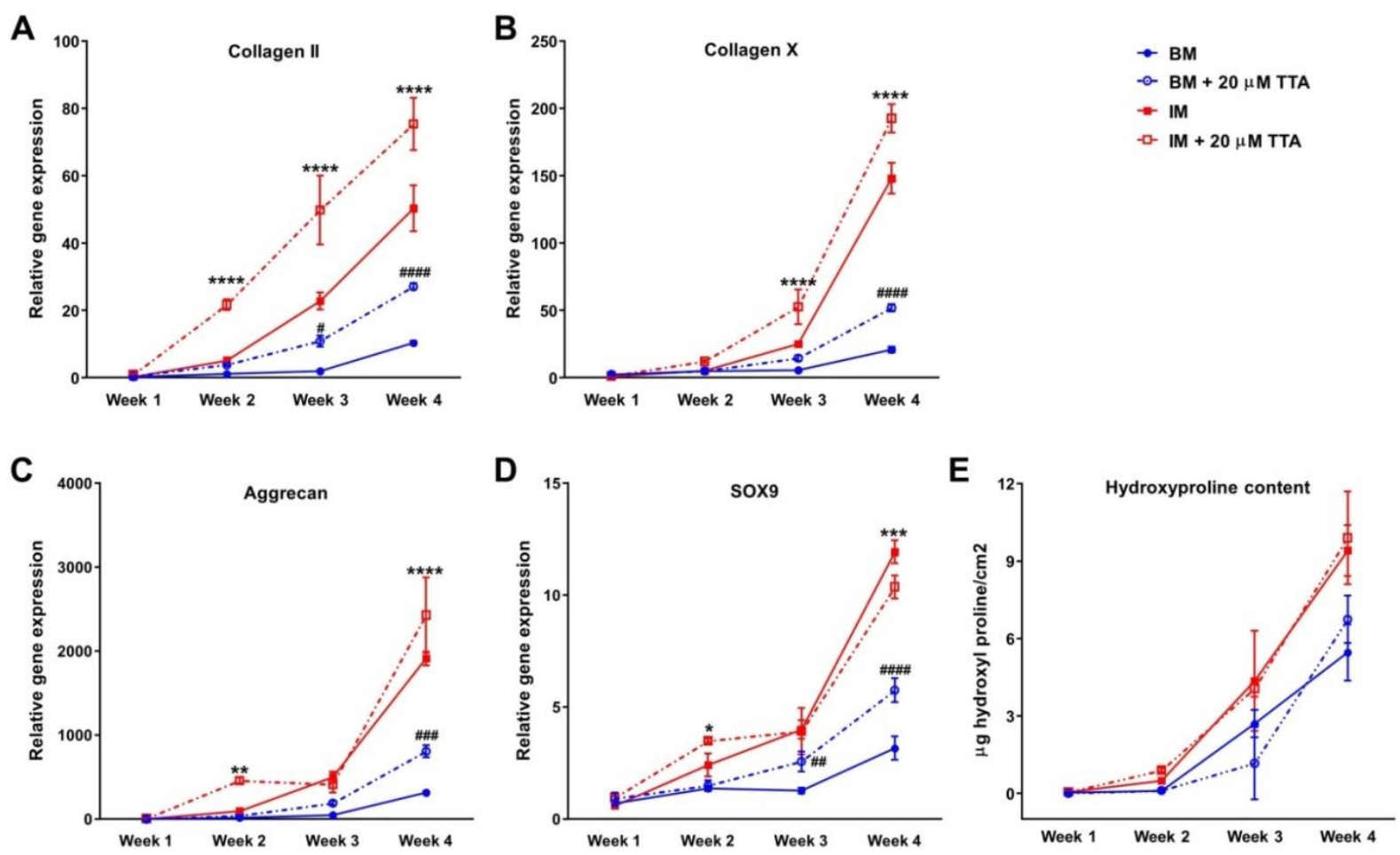

FIG. 6. The effect of TTA in long-term culture with ATDC5. Gene expression profile of collagen type II (A), collagen type X (B), aggrecan (C), and SOX9 (D), plus hydroxyproline content (E) of ATDC5 cultured on TCP in BM (solid blue line), BM supplement with 20 mM TTA (broken blue line), IM (solid red line), and IM supplemented with $20 \mathrm{mM}$ TTA (broken red line) at weeks 1, 2,3 , and 4 . Error bars represent standard deviation $(n=3)$. Statistical analysis was performed using two-way ANOVA and Bonferroni's post-test. (*) denotes a significant difference compared with the $\mathrm{BM}$ at the same time point and (\#) denotes a significant difference compared with the IM at the same time point. $(*)$ or $(\#)$ denotes $\mathrm{p}<0.05,(* *)$ or $(\# \#)$ denotes $\mathrm{p}<0.01,(* * *)$ or $(\# \# \#)$ denotes $\mathrm{p}<$ 0.001 , and $(* * * *)$ or $(\# \# \#)$ denotes $\mathrm{p}<0.0001$.

\section{Effect of TTA on other cell types}

We confirmed that TTA was capable of enhancing cECM production and inducing collagen type II and aggrecan expression in ADTC5 cells. Thus, we decided to test the effect of TTA on other cell types to see whether its effect is cell-type dependent. For this purpose, we chose human primary articular chondrocytes (hPCs) and hMSCs, the two most clinically relevant cell types for cell-based therapies aimed at cartilage repair. Three different donors were tested for each cell type to correct for donor variability. Basic and chondrogenic media were used as negative and positive controls, respectively. The concentration of TTA used was $10 \mathrm{mM}$, determined by preliminary experiments using metabolic activity assay to exclude toxic concentrations (data not shown). Since hPCs are known to produce cECM in culture, we grew them on tissue culture plastic for 14 days, and then performed gene expression analysis for 
collagen type II, aggrecan, and SOX9. hMSCs were chondrogenically differentiated in pellet cultures for 30 days, followed by gene expression analysis also on the same set of genes. For hPCs, $10 \mathrm{mM}$ TTA combined with chondrogenic medium increased the expression of aggrecan in all three donors (statistically significant in two out of three donors).

However, either used alone or in combination with chondrogenic medium, TTA downregulated the expression of collagen type II and SOX9 in this cell type (Fig. 7A). Interestingly, the same trend was seen in hMSCs when $10 \mathrm{mM}$ TTA significantly downregulated collagen type II and SOX9 expression in two of three donors (Fig. 7B). In the other donor (D301), collagen type II and SOX9 expression was upregulated (not significantly), and aggrecan expression increased 30-fold compared with the negative control when TTA was added. However, when combined with chondrogenic medium, TTA always reduced expression of these genes compared with the chondrogenic medium condition.

A

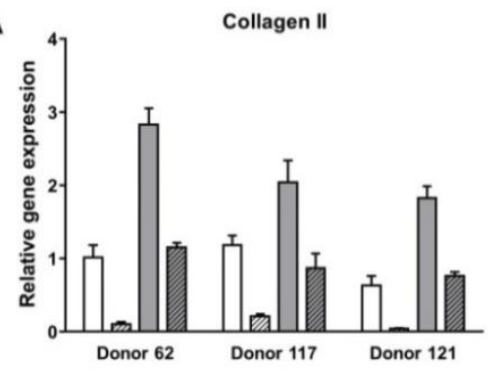

B

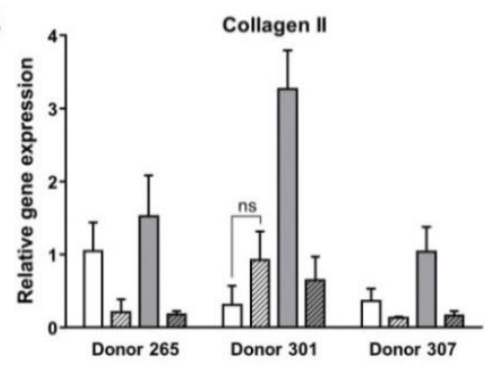

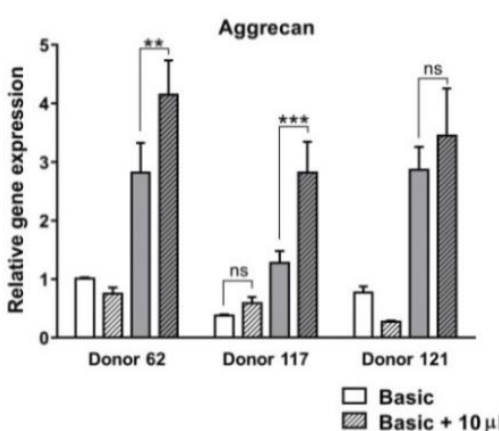
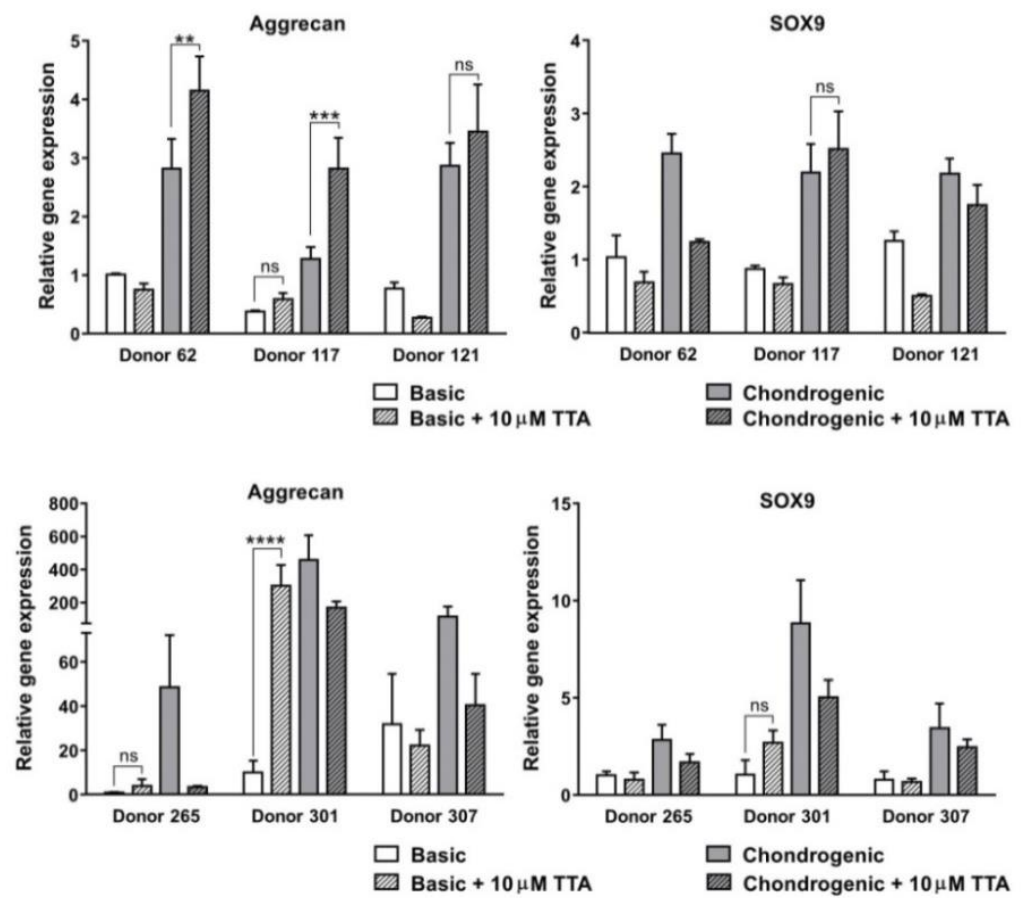

FIG. 7. Effect of TTA on human primary chondrocytes and human mesenchymal stromal cells. (A) hPCs (three donors) were cultured on TCP in chondrocyte basic medium (CBM), CBM+ 10 mM TTA, chondrogenic differentiation medium (CDM), and CDM+ $10 \mathrm{mM}$ TTA. Gene expression analysis on collagen type II, aggrecan, and SOX9 was performed after 14 days in culture. (B) hMSCs (three donors) were cultured in pellets of 2.5 105 cells in hMSC basic medium (MBM), MBM+ $10 \mathrm{mM}$ TTA, hMSC chondrogenic differentiation medium (MCM), and MCM+ $10 \mathrm{mM}$ TTA. Gene expression analysis on collagen type II, aggrecan, and SOX9 was performed after 30 days in culture. Error bars represent standard deviation ( $n=3$ for each donor). Statistical analysis was performed using one-way ANOVA and Bonferroni's post-test for each donor. (**) denotes $\mathrm{p}<$ $0.01,(* * *)$ denotes $\mathrm{p}<0.001$, and $(* * * *)$ denotes $\mathrm{p}<0.0001, \mathrm{~ns}$, nonsignificant. 


\section{Discussion}

Designing and performing an HTS requires that a number of issues are addressed. In this screen, we chose insulin as a positive control because insulin is widely used to induce ECM secretion of ATDC5 cells. Using total fluorescent intensity of the collagen probe as a readout, even insulin was only able to induce a 1.5 -fold, in total, fluorescence intensity. Still, of the six hits selected for validation from the recent screen, DL-Cycloserine and TTA could reproduce the positive response observed with insulin, although to a lesser extent. In later validation experiments, using a more sensitive technique such as qPCR with higher concentration and longer exposure time, we were able to redeem TTA as a true enhancer of cECM expression for ATDC5 cells. This could not have been done in the actual screen since all compounds were only tested at one concentration and one exposure time.

Our screen was designed to be exploratory because a universal mechanism for inducing cECM protein production is not known. For this purpose, we chose a nonspecific readout: total amount of collagen estimated by total fluorescence intensity from a collagen probe staining, which is not specific for any type of collagen. The most advantageous point of the collagen probe staining is its ease of use and thus is ideal for an HTS. The three step protocol, adding the dye, washing, and quantifying, can be performed in any laboratory manually or automatically. Its main problem is the instability of the fluorophore; even though Alexa Fluor is very good, photo bleaching will always occur. We addressed this problem by performing the staining and quantification in batches and always included both the positive and negative controls on every plate. Since the collagen probe recognizes the amino acid sequence of collagens [16, 21], it will also bind to them in solution. However, the complex remains in solution and will be removed in the washing step. The collagen probe can also bind to collagen gel (with chopped fiber) and gelatin (denatured collagen), thus it is not suitable to use in a gel system.

Other methods that can be used to quantify collagen include hydroxyproline assay and Picro Sirius red assay. While the hydroxyproline is by far the most common method for quantifying tissue fibrosis and collagen deposition [22], its laborious protocol hinders its high-throughput capability. The hydrolysis step, which is necessary for the release of amino acid hydroxyproline from the protein collagen, requires high temperature and high pressure and thus is not suitable for normal plastic well plates. With some modifications, the Picro Sirius red staining can be used in an HTS very similar to our collagen probe assay [23]. When bound to collagen, the Sirius red-collagen complex will precipitate, making it suitable for quantifying even soluble collagens.

While we confirmed that TTA is capable of enhancing cECM production by ATDC5 cells, we did not attempt to study the mechanism behind it. To date, TTA is a well-known synthetic fatty acid and is the focus of many studies for its effect on fat metabolism-related disorders [24], inflammation [25], and even cardiovascular function [26, 27]. A lot of evidence points at the involvement of TTA in the peroxisome proliferator- activated receptor (PPAR)-mediated signaling [28-30]. 
According to Forman et al. [30], TTA belongs to a group of dual-function PPAR $\alpha$ activators, which also includes tetradecylthioproprionic acid (TTP), tetradecylglycidic acid (TDGA), octylthioproprionic acid (OTP), nonylthioacetic acid (NTA), LY 171883, and 2bromopalmitate (2Br-C16). All these compounds activate PPAR $\alpha$ directly as ligands; plus, as metabolic inhibitors of the mitochondrial b-oxidation pathway, they cause accumulation of endogenous fatty acids, which are also PPAR $\alpha$ ligands. Some in vitro researches have shown the anti-proliferative effect of TTA on certain cell types [31-33] which was not confirmed by our data. Exposure of ATDC5 cells, hMSCs, and hPCs to TTA did not have a noticeable negative effect on proliferation unless very high concentrations of TTA (200 mM for ATDC5, $40 \mathrm{mM}$ for hMSCs and hPCs) were used, which led to a reduction in metabolic activity, followed by cell death (Fig. 3C, middle graph).

Since ATDC5 is a chondrogenic cell line, we wanted to see the same cECM-enhancing effect of TTA on hPCs, but unexpectedly, in our experimental conditions, TTA consistently inhibited the expression of collagen II in hPCs. This was also observed for hMSCs undergoing chondrogenic differentiation. We have yet to explain this phenomenon, but the fact that ATDC5 cells can produce abundant cECM over time even in BM, suggests that the mechanism of action of TTA on ATDC5 cells is different from that on hMSCs and hPCs. Westergaard et al. showed that TTA activated all human PPAR subtypes in the ranking order PPARd > PPARa > PPARg, whereas in the experiment using mouse PPARs, TTA most efficaciously activated PPAR $\alpha$ [33]. Although this could not explain the contradictory effect that we have seen in our experiments, it showed that the mechanism of action of TTA may be different between species.

With the ECM-enhancing effect of TTA on ATDC5 cells confirmed, especially when used in combination with insulin, we obtained a new method to grow and upscale our ATDC5 cartilage model in vitro. With our initial plan of studying the endochondral ossification process using live or devitalized hypertrophic cartilage, we can now use TTA in combination with insulin to enhance the amount of tissue mass produced by ATDC5 for in vivo implantation without the need of scaffold material.

While increasing collagen quantity is advantageous for our purpose, collagen structure and organization can greatly affect property and functionality of the ECM. In previous publications $[16,17]$, we have shown the capability of the collagen probe in high-resolution imaging. Collagen organization can be visualized with the collagen probe even better than with second harmonic generation, which is currently the most effective method for viewing 3D collagen organization in tissues. However, this resolution is not high enough to visualize collagen fibril periodicity. In this article, we have yet to look at collagen structure and organization. In the near future, we will focus on using micropatterned surfaces to modify the collagen fibril organization of the ATDC5 cell layer.

In conclusion, we have developed an HTS assay using the collagen probe CNA35-AF488 to screen for compounds capable of enhancing cECM production by the cells, and as an example, we discovered TTA as a potent cECM enhancer of ATDC5. With the method we present, any laboratory can customize the screen by choosing a different target cell type (e.g., primary 
chondrocytes, hMSCs) and a different compound library; the result will be different compounds that respond well to their cell type in terms of collagen production.

\section{References for Chapter 3 (High-throughput screen)}

1. Guilak, F., et al. Control of stem cell fate by physical interactions with the extracellular matrix. Cell Stem Cell 5, 17, 2009.

2. Watt, F.M., and Hogan, B.L. Out of Eden: stem cells and their niches. Science 287, 1427, 2000.

3. Unadkat, H.V., et al. An algorithm-based topographical biomaterials library to instruct cell fate. Proc Natl Acad Sci U S A 108, 16565, 2011.

4. von der Mark, K., et al. Nanoscale engineering of biomimetic surfaces: cues from the extracellular matrix. Cell Tissue Res 339, 131, 2010.

5. Moroni, L., de Wijn, J.R., and van Blitterswijk, C.A. Integrating novel technologies to fabricate smart scaffolds. J Biomater Sci Polym Ed 19, 543, 2008.

6. Jukes, J.M., et al. Endochondral bone tissue engineering using embryonic stem cells. Proc Natl Acad Sci U S A 105, 6840, 2008.

7. Bridges, J.B., and Pritchard, J.J. Bone and cartilage induction in the rabbit. J Anat 92, 28, 1958.

8. Urist, M.R., and Mc, L.F. Osteogenetic potency and newbone formation by induction in transplants to the anterior chamber of the eye. J Bone Joint Surg Am 34-A, 443, 1952.

9. Urist, M.R., Wallace, T.H., and Adams, T. The function of fibrocartilaginous fracture callus. Observations on Transplants Labelled with Tritiated Thymidine. J Bone Joint Surg Br 47, 304, 1965.

10. Urist, M.R., and Adams, T. Cartilage or bone induction by articular cartilage. Observations with radioisotope labelling techniques. J Bone Joint Surg Br 50, 198, 1968.

11. Muratov, R., et al. New approach to reduce allograft tissue immunogenicity. Experimental data. Interact Cardiovasc Thorac Surg 10, 408, 2010.

12. DeLustro, F., et al. Immune responses to allogeneic and xenogeneic implants of collagen and collagen derivatives. Clin Orthop Relat Res 260, 263, 1990.

13. Alves, H., et al. High-throughput assay for the identification of compounds regulating osteogenic differentiation of human mesenchymal stromal cells. PLoS ONE 6, e26678, 2011.

14. Doorn, J., et al. A small molecule approach to engineering vascularized tissue. Biomaterials 34, 3053, 2013.

15. Shukunami, C., et al. Cellular hypertrophy and calcification of embryonal carcinomaderived chondrogenic cell line ATDC5 in vitro. J Bone Miner Res 12, 1174, 1997.

16. Krahn, K.N., et al. Fluorescently labeled collagen binding proteins allow specific visualization of collagen in tissues and live cell culture. Anal Biochem 350, 177, 2006.

17. Boerboom, R.A., et al. High resolution imaging of collagen organisation and synthesis using a versatile collagen specific probe. J Struct Biol 159, 392, 2007.

18. Saito, T., et al. Transcriptional regulation of endochondral ossification by HIF-2alpha during skeletal growth and osteoarthritis development. Nat Med 16, 678, 2010.

19. Alves, H., et al. Effect of antioxidant supplementation on the total yield, oxidative stress levels, and multipotency of bone marrow-derived human mesenchymal stromal cells. Tissue Eng Part A 19, 928, 2013. 
20. Hendriks, J.A., et al. Primary chondrocytes enhance cartilage tissue formation upon coculture with a range of cell types. Soft Matter 6, 5080, 2010.

21. Patti, J.M., Boles, J.O., and Hook, M. Identification and biochemical characterization of the ligand binding domain of the collagen adhesin from Staphylococcus aureus. Biochemistry 32, 11428, 1993.

22. Woessner, J.F., Jr., The determination of hydroxyproline in tissue and protein samples containing small proportions of this imino acid. Arch Biochem Biophys 93, 440, 1961.

23. Kliment, C.R., et al. A novel method for accurate collagen and biochemical assessment of pulmonary tissue utilizing one animal. Int J Clin Exp Pathol 4, 349, 2011.

24. Berge, R.K., et al. Metabolic effects of thia fatty acids. Curr Opin Lipidol 13, 295, 2002.

25. Bjorndal, B., et al. Tetradecylthioacetic acid attenuates inflammation and has antioxidative potential during experimental colitis in rats. Dig Dis Sci 58, 97, 2013.

26. Pettersen, R.J., et al. Effects of local delivery of tetradecylthioacetic acid within the injured coronary vessel wall. Scand Cardiovasc J 46, 366, 2012.

27. Oie, E., et al. Tetradecylthioacetic acid increases fat metabolism and improves cardiac function in experimental heart failure. Lipids 48, 139, 2013.

28. Wrzesinski, K., et al. Proteomics identifies molecular networks affected by tetradecylthioacetic acid and fish oil supplemented diets. J Proteomics 84, 61, 2013.

29. Bocos, C., et al. Fatty acid activation of peroxisome proliferator-activated receptor (PPAR). J Steroid Biochem Mol Biol 53, 467, 1995.

30. Forman, B.M., Chen, J., and Evans, R.M. Hypolipidemic drugs, polyunsaturated fatty acids, and eicosanoids are ligands for peroxisome proliferator-activated receptors alpha and delta. Proc Natl Acad Sci U S A 94, 4312, 1997.

31. Lundemo, A.G., et al. Tetradecylthioacetic acid inhibits proliferation of human SW620 colon cancer cells - gene expression profiling implies endoplasmic reticulum stress. Lipids Health Dis 10, 190, 2011.

32. Tronstad, K.J., et al. Antiproliferative effects of a non-betaoxidizable fatty acid, tetradecylthioacetic acid, in native human acute myelogenous leukemia blast cultures. Leukemia 16, 2292, 2002.

33. Westergaard, M., et al. Modulation of keratinocyte gene expression and differentiation by PPAR-selective ligands and tetradecylthioacetic acid. J Invest Dermatol 116, 702, 2001. 
A

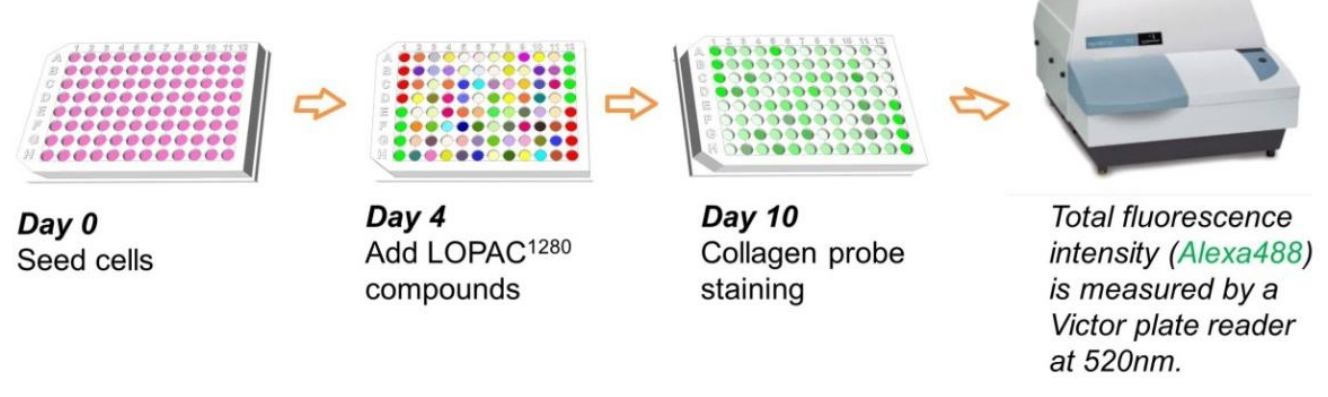

B

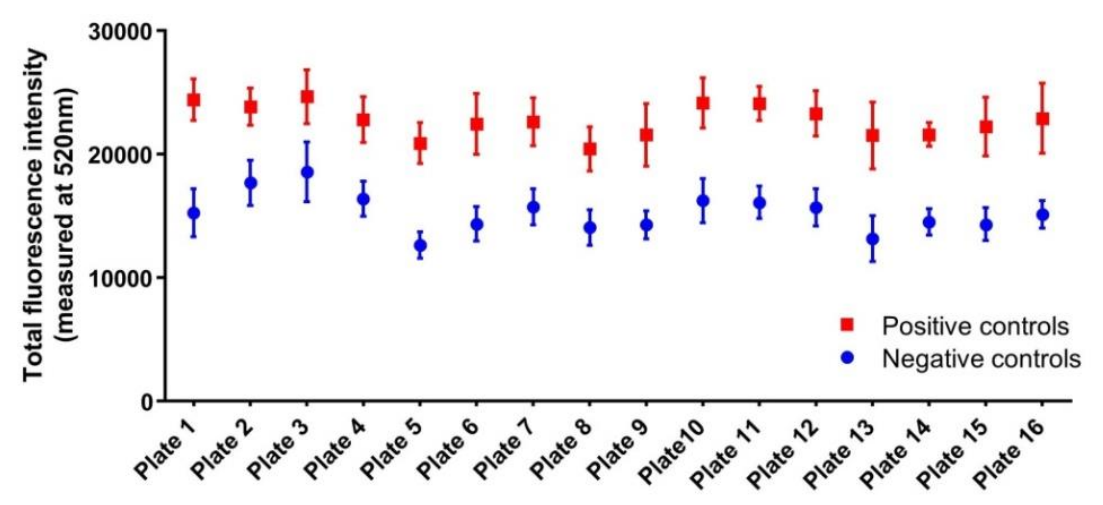

Supp FIG. 1. Schematic of the high throughput screening assay using collagen probe CNA35Alexa488. (A) At day 0, cells were seeded at 5,000 cells $/ \mathrm{cm}^{2}$ on 96 -well clear imaging plates. At day 4, the library of compounds (LOPAC1280) was added to the testing wells; IM-positive controls (PC) and BM-negative controls (NC) are included in every plate. At day 10, the collagen probe staining was performed. After 1 hour of incubation, the plates were washed thrice with PBS. Next, total fluorescence intensity was measured using a Victor plate reader. (B) Per plate analysis of the controls. The Figure represents the effect of PC and NC on ECM production of ATDC5 cell as quantified by the collagen probe assay. Error bars represent standard deviation $(n=8)$. 
Supp. Table 1. Hit compounds identified after the screen

\begin{tabular}{|c|c|c|c|c|c|c|}
\hline Plate & $\begin{array}{c}\text { Mean NC } \\
+3 \times \text { S.D. }\end{array}$ & Mean PC & $\begin{array}{c}\text { Hit } \\
\text { signal }\end{array}$ & Well & \multicolumn{2}{|c|}{ Sigma Aldrich catalogue number and compound name } \\
\hline \multirow[t]{3}{*}{1} & 21044.42 & 24426.75 & 22306 & B10 & Т 9034 & Taucholic acid \\
\hline & & & 21128 & E7 & A 3846 & AL-8810 \\
\hline & & & 21895 & E10 & A 6134 & Arecoline hydrobromide \\
\hline \multirow[t]{2}{*}{2} & 22345.51 & 23839.13 & 22368 & D6 & A 9950 & Aniracetam \\
\hline & & & 22795 & D10 & A- 254 & AIDA \\
\hline 3 & 25093.63 & 24668.25 & No hit & & & \\
\hline 4 & 20671.06 & 22795.88 & 21164 & $\mathrm{C} 8$ & C 7005 & DL-Cycloserine \\
\hline 5 & 15831.06 & 20895.88 & 17302 & G9 & D 4268 & $\mathrm{~N}^{\wedge} \mathrm{G}, \mathrm{N}^{\wedge} \mathrm{G}$ Dimethylarginine Hydrochloride \\
\hline 6 & 18545.25 & 22447.50 & No hit & & & \\
\hline 7 & 20072.17 & 22625.13 & No hit & & & \\
\hline 8 & 18405.58 & 20435.75 & 18527 & F8 & L 2167 & L-165,041 \\
\hline 9 & 17661.89 & 21573.75 & 18291 & D10 & L 5783 & Lidocaine N-ethyl bromide quaternarysalt \\
\hline 10 & 21595.37 & 24139.88 & 21945 & D4 & M 1559 & Moxonidine hydrochloride \\
\hline 11 & 20028.86 & 24096.13 & No hit & & & \\
\hline 12 & 20255.27 & 23285.75 & No hit & & & \\
\hline 13 & 18678.36 & 21521.13 & No hit & & & \\
\hline 14 & 17718.56 & 21597.75 & No hit & & & \\
\hline 15 & 18300.09 & 22231.38 & 18398 & A11 & T 6764 & Triprolidine hydrochloride \\
\hline & & & 21115 & D6 & Т 1698 & Tetradecyl thioacetic acid \\
\hline & & & 18806 & G7 & T 4500 & Theobromine \\
\hline 16 & 18435.89 & 22900.50 & No hit & & & \\
\hline
\end{tabular}


Supp. Table 2. Primer sequences

\begin{tabular}{ll}
\hline Name & Primer sequence \\
\hline B2m: beta-2 microglobulin [mouse] & 5'-CATGGCTCGCTCGGTGACC-3' \\
& 5'-AATGTGAGGCGGGTGGAACTG-3' \\
Col2a1: collagen type II alpha 1 [mouse] & 5'-CAAGGCCCCCGAGGTGAAAA-3' \\
& 5'-GGGGCCAGGGATTCCATTAGAGC-3' \\
Col10a1: collagen type X alpha 1 [mouse] & 5'-CATAAAGGGCCCACTTGCTA-3' \\
& 5'-TGGCTGATATTCCTGGTGGT-3' \\
Acan: aggrecan [mouse] & 5'-AGAACCTTCGCTCCAATGACTC-3' \\
& 5'-AGGGTGTAGCGTGTGGAAATAG-3' \\
Sox9: SRY-box9 [mouse] & 5'-CCACGGAACAGACTCACATCTCTC-3' \\
& 5'-CTGCTCAGTTCACCGATGTCCACG-3' \\
B2M: beta-2 microglobulin [human] & 5' GACTTGTCTTCAGCAAGGA 3' \\
& 5' ACAAAGTCACATGGTTCACA 3' \\
COL2A1: collagen type II alpha 1 [human] & 5' CGTCCAGATGACCTTCCTACG 3' \\
& 5' TGAGCAGGGCCTTCTTGAG 3' \\
ACAN: aggrecan [human] & 5' AGAATCCACCACCACCAG 3' \\
& 5' ATGCTGGTGCTGATGACA 3' \\
SOX9: SRY-box9 [human] & 5' TGGGCAAGCTCTGGAGACTTC 3' \\
& 5' ATCCGGGTGGTCCTTCTTGTG 3' \\
\hline
\end{tabular}




\title{
Chapter 4
}

\section{An approach to in vitro manufacturing of hypertrophic cartilage matrix for bone repair}

\author{
Bach Q. Le, Clemens van Blitterswijk, Jan de Boer
}

$\mathrm{D}$

evitalized hypertrophic cartilage matrix (DCM) is an attractive concept for an off-theshelf bone graft substitute. Upon implantation, DCM can trigger the natural endochondral ossification process, but only when the hypertrophic cartilage matrix has been reconstituted correctly. In vitro hypertrophic differentiation has been reported for multiple cell types but up-scaling and in vitro devitalization remain a big challenge. To this end, we developed a micro tissue-engineered cartilage (MiTEC) model using the chondrogenic cell line ATDC5. Micro-aggregates of ATDC5 cells (approximately 1000 cells per aggregate) were cultured on a $3 \%$ agarose mold consisting of 1585 microwells, each measuring $400 \mu \mathrm{m}$ in diameter. Chondrogenic differentiation was strongly enhanced using media supplemented with combinations of growth factors e.g. insulin, transforming growth factor beta and dexamethasone. Next, mineralization was induced by supplying the culture medium with betaglycerophosphate, and finally we boosted the secretion of proangiogenic growth factors using the hypoxia mimetic phenanthroline in the final stage of in vitro culture. Then, ATDC5 aggregates were devitalized by freeze/thawing or sodium dodecyl sulfate treatment before coculturing with human mesenchymal stromal cells (hMSCs). We observed a strong effect on chondrogenic differentiation of hMSCs. Using this MiTEC model, we were able to not only upscale the production of cartilage to a clinically relevant amount but were also able to vary the cartilage matrix composition in different ways, making MiTEC an ideal model to develop DCM as a bone graft substitute. 


\section{Introduction}

Endochondral ossification is a fascinating phenomenon in which a cartilage template is remodeled into bone tissue by a highly regulated mechanism. During embryonic development, endochondral ossification occurs in parallel to intramembranous ossification to build the skeletal system. In fracture healing, the process very much recapitulates embryonic development and thus usually results in complete restoration of the skeletal organ [1-3]. However, when bone defects reach critical sizes or when systemic factors such as osteoporosis presents, clinical intervention is necessary to augment bone healing [4]. Current options to treat bone defects ranges from autologous bone to synthetic osteoinductive materials with/without growth factors e.g. bone morphogenetic proteins [5]. Until now, there is no clinical treatment that utilizes endochondral ossification, specifically from a hypertrophic cartilage graft.

Previously, we have demonstrated the feasibility to tissue-engineer hypertrophic cartilage from mouse embryonic stem cells (mESCs) in vitro which then remodeled into new bone in vivo [6]. To this end, mESCs were chondrogenically differentiated for 2 to 3 weeks, followed by subcutaneous implantation in immunodeficient mice for 4 weeks. A high correlation was observed between the amount of hypertrophic cartilage created in vitro and the amount of bone detected in vivo. Similarly, Scotti et al. subcutaneously implanted bone marrow-derived human mesenchymal stromal cells (hMSCs) at various stages of chondrogenic differentiation [7]. They observed bone trabeculae only when the hMSCs differentiated in vitro into hypertrophic cartilage-like tissue. Weiss et al. differentiated ATDC5 pellets into hypertrophic cartilage in vitro before implantation in nude mice for 8 weeks [8]. They found mineralized tissue with active osteoclast resorption and neo-angiogenesis throughout the implants. These experiments and others [9-12] give proof of concept that in vitro cultured hypertrophic cartilage can continue endochondral ossification in vivo.

Live hypertrophic cartilage will form bone in vivo, but it has long been realized that even non-living tissues still contain bioactive components that can induce ossification. The most relevant example is demineralized bone matrix (DBM) developed by Urist in 1960. DBM is allogenic or xenogeneic bone processed to remove immunogenicity and preserve the osteoinductive bone morphogenetic proteins [13]. The chemistry of the remaining extracellular matrix (ECM), not the living cells, is sufficient to induce bone formation upon implantation and DBM is widely used as a bone graft substitute. Interestingly, hypertrophic cartilage tissue which is devitalized under certain conditions can also form bone in vivo. Bridge and Pritchard showed that epiphyseal plate or fracture callus devitalized with alcohol, acetone, $\mathrm{HCl}$ or heated to $55^{\circ} \mathrm{C}$ consistently formed bone when implanted subcutaneously in the ear of rabbits [14].

Urist also observed bone formation when devitalized fracture callus was implanted into the anterior chamber of the eye of rats [15-17]. More recently, Bourgine et al. proved that hMSCderived hypertrophic cartilage which was devitalized by inducible apoptosis could efficiently remodel to form de novo bone tissue of host origin, including mature vasculature and a hematopoietic compartment [18]. Cunniffe et al. created a porous scaffold by freeze-drying SDS-decellularized hMSC-engineered hypertrophic cartilage tissue. The scaffold induced vascularization and de novo mineral accumulation in a mouse ectopic model and formed full bridging (4 out of 8 animals) in a rat critically-sized femoral defect model [19]. Taken all 
together, these experiments demonstrated the feasibility of an osteoinductive material made of devitalized hypertrophic cartilage matrix (DCM), either harvested from biological sources or cultured in the lab.

A problem that will eventually hinder utilization of DCM in the clinic is to manufacture a great amount of this material in a reproducible and cost-effective manner. Making DCM from allografts or xenografts will not yield sufficient amount since hypertrophic cartilage only present in small amount at the ends of long bones before adulthood. Thus, producing DCM from hypertrophic cartilage cultured in vitro seems to be a plausible answer. For this matter, choosing a cell type is the most critical step. One could start with undifferentiated stem cells, for examples ES cells or hMSCs, then induce chondrogenic differentiation until the cells reach hypertrophic stage. The disadvantages of stem cells are, apart from the cost of maintaining stemness during storage and expansion, chondrogenic differentiation of ES cells is often heterogeneous [20] and donor variation in hMSCs is inevitable [21]. Starting with mature cells such as chondrocytes poses another problem; the cells have limited proliferation capacity before becoming senescent. While the choice of cell types remains to be elucidated, it is worth mentioning that the ideal cell type should have the following properties: (1) already committed to the chondrogenic lineage and (2) have unlimited capacity to divide.

In the current study, although we don't claim to have the ideal cell type, we chose a cancer cell line -ATDC5- which has both mentioned properties to demonstrate our hypertrophic cartilage in-vitro culturing model. The cell line ATDC5, derived from a mouse teratocarcinoma, is an excellent model to study skeletal development and has been utilized in over 200 studies to date [22]. ATDC5 has an intrinsic property to sequentially undergo hypertrophic chondrogenic differentiation [22-24]. Previously, we developed a spheroid culture system which allows us to assemble 3D, free-standing tissues with an intermediate complexity between 2D cell culture and model organisms [25]. The advantages of this system and its application to date has been reviewed by Fennema et al [26].

Here, we cultured ATDC5 in aggregates of approximately 1,000-10,000 cells, which we named Micro Tissue-Engineered Cartilage (MiTEC). Compared to 2D culture models, MiTEC offers better cell-cell contacts and chondrogenic differentiation. Compared to traditional micromass or pellet models (of about 100,000 to 1,000,000 cells), MiTEC allows better diffusion of nutrients and growth factors. This model does not require additional scaffolding material but can be easily upscaled to produce a large amount of tissue mass applicable for all clinical purposes. Our long-term goal is to create an osteoinductive material made of devitalized ATDC5-derived hypertrophic cartilage that offers the ideal micro-environment for en route endochondral ossification. To reach this goal, in this study we optimized the in vitro production of MiTEC qualitatively and quantitatively. Medium composition and culture time was optimized to maximize hypertrophy; endogenous growth factor (VEGF) secretion was enhanced by treatment with hypoxia mimic molecules; and mineralization was induced with betaglycerophosphate. MiTEC were devitalized either by freeze/thawing or by sodium dodecyl sulfate (SDS) treatment. Upon co-culturing with hMSCs, the SDS treated MiTEC had a strong effect on hMSC chondrogenic differentiation. Thus, we demonstrated the usability of the MiTEC model in optimizing and upscaling the in vitro culture of hypertrophic cartilage. 


\section{Materials and methods}

Cell culture

ATDC5 cells (RIKEN cell bank - Japan) were maintained in basic medium (BM) consisting of DMEM/F-12 GlutaMAX, 5\% v/v fetal bovine serum (FBS, Lonza), $0.2 \mathrm{mM}$ L-ascorbic acid2 phosphate (ASAP), $100 \mathrm{U} / \mathrm{mL}$ penicillin and $100 \mu \mathrm{g} / \mathrm{mL}$ streptomycin (PS). Cells were grown at $37^{\circ} \mathrm{C}$ in a humid atmosphere with $5 \% \mathrm{CO}_{2}$. Medium was refreshed 2-3 times per weeks and cells were used for further subculturing or cryopreservation upon reaching near confluence.

3\% agarose (Life Technologies) chips were produced by replica molding from elastomeric stamps of poly(dimethylsiloxane) (PDMS; Sylgard 184, Dow Corning); the stamps were replicated from either etched silicon wafers or SU-8/silicon wafers [25]. An agarose chip contains 1585 micro-wells, each measuring $400 \mu \mathrm{m}$ in diameter and fitting in a well of a 12well plate. To make aggregates, 1.5 million cells were seeded per agarose chip and centrifuged for 1 minute at $300 \mathrm{G}$ to collect the cells at the bottom of the micro-wells. This seeding density created an aggregate size of about 1,000 cells per aggregate, which was optimized for our 400 $\mu \mathrm{m}$ diameter micro-wells. Medium was changed every 1-2 days by pipetting from the side of the agarose chips without disturbing the aggregates. To optimize medium composition, 4 different formulations were used: (1) basic medium (BM); (2) BM supplemented with 1x insulin-transferrin-selenium (ITS) solution (IM); (3) BM supplemented with $0.1 \mu \mathrm{M}$ dexamethasone (Dex) and $10 \mathrm{ng} / \mathrm{mL}$ transforming growth factor 3 (TGF 33 , R\&D systems, 243B3-010) (BC); and BM supplemented with 1x ITS, $0.1 \mu \mathrm{M}$ Dex and $10 \mathrm{ng} / \mathrm{mL}$ TGF $\beta 3$ (IC).

Primary human mesenchymal stem cells (hMSCs) were obtained and isolated as described previously [27]. The use of bone marrow aspirates was approved for this whole study by Medical Ethics Committee of Medisch Spectrum Twente and written informed consent was obtained from all patients. hMSCs were expanded at an initial seeding density of 1,000 cells $/ \mathrm{cm}^{2}$ in proliferation medium consisting of $\alpha$-MEM, 10\% v/v FBS (Lonza), $2 \mathrm{mM} \mathrm{L-}$ glutamine, $0.2 \mathrm{mM}$ ASAP, PS, and $1 \mathrm{ng} / \mathrm{ml}$ recombinant human basic fibroblast growth factor (AbD Serotec). For pellet mix-culture of hMSCs with devitalized or decellularized ATDC5 aggregates, a cell suspension of $2.5 \times 10^{5} \mathrm{hMSCs}$ (passage 2-5) were mixed with aggregates taken from 1 agarose chip in a 10-ml tube (Greiner) and centrifuged for 3 minutes at $300 \mathrm{G}$ to form a pellet. As a control, $2.5 \times 10^{5} \mathrm{hMSCs}$ alone were used to form a pellet in the same way. Chondrogenic medium for pellet co-culture consisted of DMEM high glucose, $100 \mu \mathrm{g} / \mathrm{mL}$ sodium pyruvate, $0.2 \mathrm{mM}$ ASAP, PS, $1 \mathrm{x}$ ITS, $0.1 \mu \mathrm{M}$ Dex, and $10 \mathrm{ng} / \mathrm{mL}$ TGF $\beta 3$ [28]. Basic medium for pellet co-culture was chondrogenic medium without Dex and TGF $\beta 3$. The pellets were cultured for 4 weeks and medium was changed 2-3 times per week. All media and medium supplements were purchased from Life Technologies; all chemicals were purchased from Sigma-Aldrich, unless otherwise stated.

\section{Devitalization and decellularization}

ATDC5 aggregates were cultured for 2 weeks in IC medium before devitalization by liquid nitrogen freeze/thawing or decellularization by sodium dodecyl sulfate (SDS). For devitalization, aggregates were collected and washed once with PBS followed by submersion in liquid nitrogen for 30 seconds and then in a $45^{\circ} \mathrm{C}$ water bath for 30 seconds, which was 
repeated 10 times. Decellularization was based on a protocol described by Kheir et al [29]. Briefly, aggregates were subjected to 2 cycles of dry freeze/thaw followed by another 2 cycles of freeze/thaw in hypotonic buffer consisting of $10 \mathrm{mM}$ Tris- $\mathrm{HCl}(\mathrm{pH}$ 8.0) supplemented with $1 \mathrm{x}$ halt proteinase inhibitor cocktail (Thermo Scientific). Samples were frozen at $-20^{\circ} \mathrm{C}$ until crystal formation and then thawed on bench for 4 hours. Then, aggregates were incubated in hypotonic buffer, $45^{\circ} \mathrm{C}$ for $24 \mathrm{~h}$, followed by $0.1 \%$ w/v SDS in hypotonic buffer at $45^{\circ} \mathrm{C}$ for 24 $\mathrm{h}$, with agitation. After washing with PBS twice for 30 min twice and incubation for $24 \mathrm{~h}$ at $45^{\circ} \mathrm{C}$, aggregates were treated with a nuclease solution consisting of $50 \mathrm{mM}$ Tris $(\mathrm{pH} 7.5), 10$ $\mathrm{mM}$ magnesium chloride, $50 \mu \mathrm{g} / \mathrm{ml}$ bovine serum albumin, DNase (Sigma-Aldrich, $50 \mathrm{U} / \mathrm{ml}$ ) and RNase (Sigma-Aldrich, $1 \mathrm{U} / \mathrm{ml}$ ), for $3 \mathrm{~h}$ at $37^{\circ} \mathrm{C}$. Finally, the aggregates were washed in PBS twice for $30 \mathrm{~min}$ and incubated for $24 \mathrm{~h}$ at $45^{\circ} \mathrm{C}$. All chemicals were purchased from Sigma-Aldrich unless otherwise stated.

\section{Histological staining and immunostaining}

Samples were fixed in $4 \%$ paraformaldehyde (Merck) in PBS. To avoid loss during histology processing, aggregates were incorporated in $0.5 \%$ agarose. Molten agarose $(0.5 \%$ in $\mathrm{ddH}_{2} \mathrm{O}$ ) was poured into $10 \mathrm{ml}$ tubes containing aggregates followed by a quick centrifugation to collect all the aggregates at the bottom of the tubes. After solidification, the agarose portions holding all the aggregates were cut off for processing. Samples were dehydrated using sequential ethanol series, embedded in paraffin and $7 \mu \mathrm{m}$ sections were cut using a microtome. Cartilage formation was visualized by $1 \%$ Alcian Blue staining in $3 \%$ acetic acid and $0.1 \%$ Nuclear Fast Red in 5\% aluminum sulfate, which stained sulfated glycosaminoglycans blue and cell nuclei red. Mineralization was visualized by $2 \%$ Alizarin Red S, which stained calcium deposits orange-red. All chemicals were purchased from Sigma-Aldrich unless otherwise stated.

For immunostaining, the VECTASTAIN@ Elite ABC-Peroxidase kit (Vector laboratories, Burlingame, CA, USA) was employed following the manufacturer's protocol with some modifications. Briefly, antigen retrieval was performed by incubating sections with $0.1 \% \mathrm{w} / \mathrm{v}$ hyaluronidase (Sigma, H3506) and 0.1\% w/v protease (Sigma, P5147) in PBS for $30 \mathrm{~min}$ at $37^{\circ} \mathrm{C}$. Sections were washed with $0.1 \%$ Tween-PBS and blocked with $0.3 \% \mathrm{H}_{2} \mathrm{O}_{2}$-PBS for 10 min followed by $5 \%$ BSA-PBS for $30 \mathrm{~min}$ both at room temperature. Sections were incubated with primary antibody anti-collagen type II (1:100; Abcam; ab34712) or anti-collagen type X antibody (1:100; Abcam; ab58632), overnight at $4^{\circ} \mathrm{C}$ in a humidified chamber. After washing, sections were incubated with biotinylated secondary antibody (1:100) followed by VECTASTAIN® ABC Reagent for $30 \mathrm{~min}$ each at RT. Brown staining was developed by incubating sections with peroxidase substrate solution for $5 \mathrm{~min}$. Sections were rinsed with tap water and counterstained with haematoxylin (Sigma). Histological sections were analyzed by light microscopy (E600 Nikon).

\section{Mineralization, calcium assay and hydroxyproline assay}

Aggregates were cultured in IC medium for 2 or 3 weeks before switching to mineralization medium consisting of $\alpha$-MEM, 5\% v/v FBS, PS, 1x ITS, and $10 \mathrm{mM} \beta$-glycerophosphate. After 1 week culture in mineralization medium, aggregates were collected for histological analysis, hydroxyproline assay and calcium assay. Samples were transferred to pressure-tight, teflon capped vials and hydrolyzed with $100 \mu \mathrm{l}$ of $12 \mathrm{M} \mathrm{HCl}$ for 3 hours at $120^{\circ} \mathrm{C} .5 \mu \mathrm{l}$ of the lysate 
was used for calcium analysis using to the QuantiChrom Calcium Assay Kit (DICA-500) according to the manufacturer's protocol. Briefly, free calcium specifically forms a stable blue colored complex with the phenolsulphonephthalein dye in the kit. The color intensity, measured at $612 \mathrm{~nm}$, is directly proportional to the calcium concentration in the sample. A standard curve was generated using $\mathrm{Ca}^{2+}$ standard. For hydroxyproline analysis, the Hydroxyproline Assay Kit (Biovision-K555) was used. Briefly, 10 $\mu$ l of the hydrolyzed sample lysate was transferred to a 96-well plate and evaporated to dryness under vacuum. Next, chloramine $\mathrm{T}$ and 4(dimethylamino)benzaldehyde (DMAB) reagent was added to each sample and incubated for $90 \mathrm{~min}$ at $60^{\circ} \mathrm{C}$. The reaction resulted in a colorimetric $(560 \mathrm{~nm})$ product, proportional to the hydroxyproline present. A standard curve was generated using 4-hydroxyproline as indicated in the manufacturer's protocol.

\section{Gene expression analysis}

Cell aggregates or pellets were collected and washed once with PBS and then homogenized in Trizol reagent (Life Technologies) by crushing with a pestle and mortar under liquid nitrogen. After adding $20 \% \mathrm{v} / \mathrm{v}$ chloroform and centrifugation for 15 minutes at $11,000 \mathrm{G}$ at $4^{\circ} \mathrm{C}$, the aqueous phase containing RNA was transferred to a new Eppendorf tube, combined with an equal volume of $70 \%$ ethanol, and then loaded onto the RNA binding column of the RNA II nucleospin RNA isolation kit (Machery Nagel). The rest of the RNA isolation followed the kit protocol. RNA concentrations were measured using a ND100 spectrophotometer (Nanodrop1000). cDNA was synthesized from $1 \mu \mathrm{g}$ of RNA, using iScript (BioRad) according to the manufacturer's protocol. qPCR was performed using $50 \mathrm{ng}$ of cDNA, $0.4 \mu \mathrm{M}$ of each forward and reverse primer (Sigma Genosys), and 1x SensiMix SYBR \& Fluorescein master mix (Bioline). Primer sequences are shown in Table 1. Real-time qPCR was performed in a Biorad My IQ5 machine (Biorad). Data was analyzed using the fit point method of My IQ5 software. The baseline was calculated automatically by the software at the lower log-linear part above baseline noise and the crossing temperature ( $\mathrm{Ct}$ value) was determined. $\mathrm{Ct}$ values were normalized to the Beta-2 microglobulin (B2M) housekeeping gene and $\Delta \mathrm{Ct}(\mathrm{Ct}$, control $-\mathrm{Ct}$, sample) was used to calculate the up-regulation in gene expression.

\section{Enzyme-linked immunosorbent assay (ELISA)}

ATDC5 aggregates were cultured for 2 or 3 weeks in different media (BM, IM, BC or IC). Medium was changed every 2 days, with only half of the volume $(1 \mathrm{~mL})$ changed to avoid loss of the aggregates. VEGF was allowed to accumulate in medium for 2 days before the collection point. To boost VEGF secretion, ATDC5 aggregates were cultured in IC medium for 2 or 3 weeks, then medium was supplemented with $50 \mu \mathrm{M}$ phenanthroline $(1,10$-Phenanthroline monohydrate, Sigma-Aldrich) [30] and VEGF was allowed to accumulate for 2 days, 3 days or 6 days (with 1 medium change after 3 days) before the collection point. $1 \mathrm{ml}$ of medium was collected from each chip and 50 $\mu$ l was assayed in duplicate using a mouse VEGF quantikine ELISA kit (R\&D, MMV00) following the kit protocol.

\section{$\underline{\text { Statistics }}$}

One-way or two-way ANOVA with Bonferroni post-test were performed using GraphPad Prism version 6.02 for Windows, GraphPad Software, La Jolla California USA. 


\section{Results}

\section{Bulk production of micro-tissue engineered cartilage (MiTEC)}

ATDC5 micro-aggregates were cultured in basic medium (BM) or basic medium supplemented with 1x ITS (IM medium) for 4 weeks on agarose chips. Cell aggregates formed within 24 hours after seeding (Fig. 1A). Over longer time, the aggregates in IM medium grew bigger and firmer than those in BM medium. After 4 weeks, the aggregates in IM medium developed a very firm appearance whereas those in BM appeared to break down. Scanning electron microscopy of aggregates culture in IM medium showed a uniform size distribution (Fig. 1B). After even longer culture time, clusters of up to 10 aggregates were observed. The exterior of these aggregates were covered by thick layers of extracellular matrix, while inside them lay many lacunae where the cells resided. Gene expression analysis of aggregates cultured in BM and IM medium for 4 weeks was performed (Fig. 1C and Supp Fig. 1A).
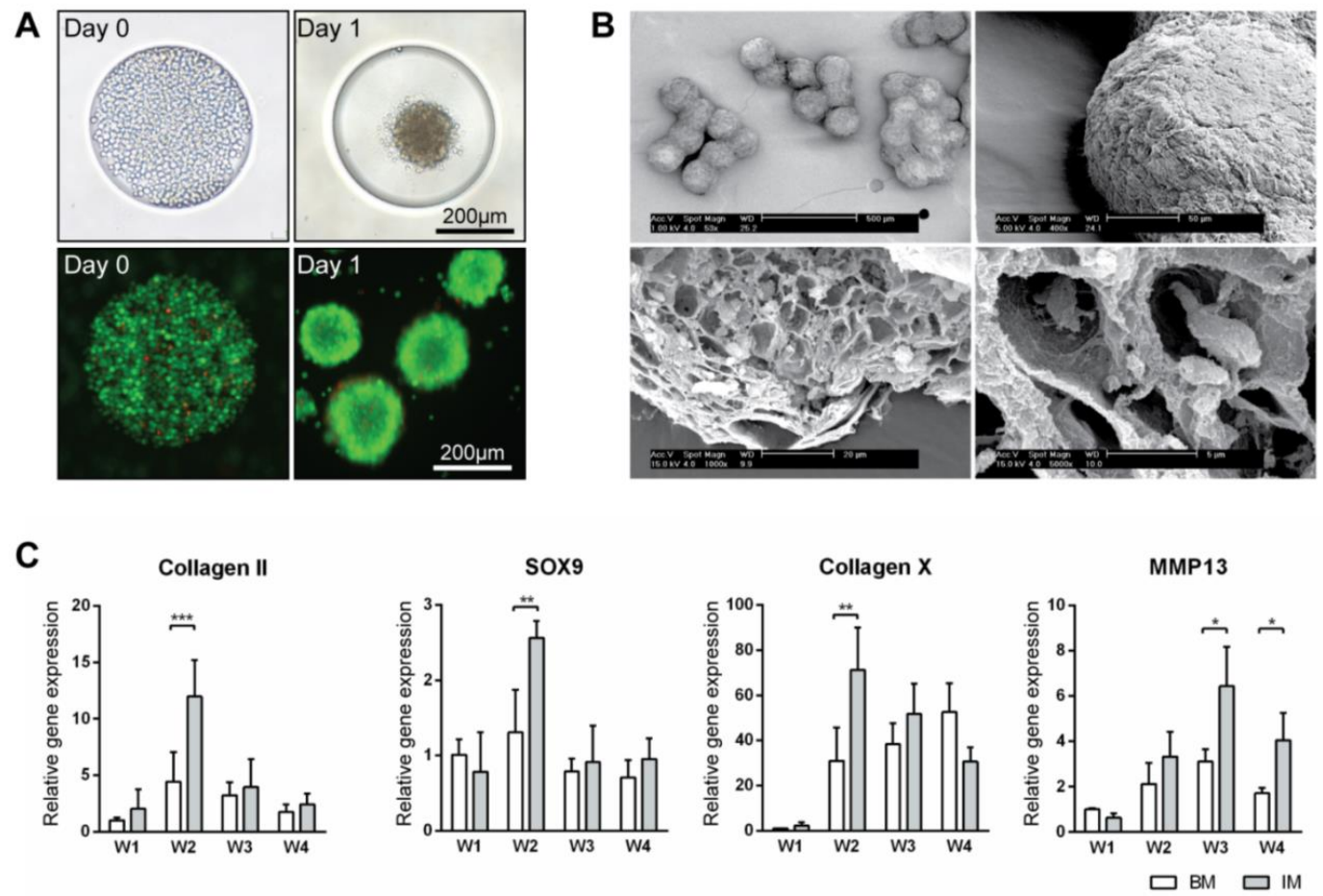

FIG. 1. Production of micro-tissue engineered cartilage (MiTEC). Cells were seeded on day 0 and the aggregate formed on day 1; the panel shows brightfield images (top row) and fluorescent images (bottom row) of cells staining with calcein (green) and ethidium homodimer-1 (red) (A). Scanning electron microscope images of MiTEC showed uniform size aggregates and cartilage-like lacunae (B). Gene expression profile of MiTEC cultured in basic medium (BM - white bar) and basic medium + ITS (IM - gray bar) at week 1-4 (C). Error bars represent standard deviation $(\mathrm{n}=3)$. $(*)$ denotes $\mathrm{p}<0.05$, (**) denotes $\mathrm{p}<0.01$, (***) denotes $\mathrm{p}<0.001$. 
Genes related to early chondrogenesis (collagen type II, aggrecan, Hif1a, and Sox9) and late chondrogenesis and hypertrophy (collagen type X, Mmp13, Hif2a, and Alp) were studied. Expression of all early markers peaked at week 2 in both BM and IM conditions, although with higher expression in IM medium, then declined in the following weeks. Hypertrophic markers collagen type X and Alp expression peaked at week 2, with expression in IM condition double that of the BM condition. Mmp13 and Hif2a expression peaked at week 3 and 4 respectively, with expression in the IM always higher than BM condition. This characterization has demonstrated the feasibility of using our spheroid culture system to grow and differentiate ATDC5 in vitro.

\section{Optimization of chondrogenic differentiation of MiTEC}

Improved chondrogenic differentiation of aggregates in IM medium showed that it is possible to manipulate the expression of chondrogenic genes. Since the amount of hypertrophic cartilage matrix is known to correlate to in vivo bone formation [6,7], we next maximized extracellular matrix (ECM) deposition in MiTEC by optimizing medium composition [28]. Four different medium compositions were tested: basic medium (BM); BM supplemented with ITS (IM); BM supplemented with TGF $\beta 3$ and Dex (BC); and BM supplemented with ITS, TGF $\beta 3$ and Dex (IC). After only 1 week in culture, the difference in size of MiTECs was apparent by light microscopy (Fig. 2, top row). In ascending order, BC, IM and IC medium had a positive effect on size of MiTEC over BM.

Histological analysis with Alcian Blue staining for glycosaminoglycan in cartilage (Fig. 2) clearly shows that BM was not a good medium to culture MiTEC, with disintegration of the aggregates and very poor Alcian Blue stain. In IM medium condition, spots with blue stain were visible in week 2 which became bigger and more intense in week 3. Hypertrophic chondrocytes, recognized by their large lacunae, appeared to reside within the intensely blue stained extracellular matrix. Interestingly, the use of both TGF $\beta 3$ and Dex (BC condition) resulted only in a faint Alcian Blue stain at both week 2 and 3. The aggregates in BC condition were also smaller in size compared IM condition (just ITS added to basic medium). Finally, when cultured in the presence of TGF $\beta 3$, Dex and ITS (IC condition), uniform and intense blue stain was observed in all aggregates.

Gene expression analysis for early and late chondrogenic markers is shown in Fig. $\mathbf{3}$ and Supp Fig. 1B. For early markers (collagen type II, aggrecan, Sox9, and Hif1a), gene expression in BC and IC conditions (both contained TGF $\beta 3$ and Dex) was always higher than those of BM and IM conditions (except for Hif1a where expression in BC condition was similar to those of $\mathrm{BM}$ and IM). For late markers, collagen type $\mathrm{X}$ expression in the $\mathrm{BC}$ and IC conditions was particularly high (300 times at week 2 and 500-1500 times at week 3) compared to BM and IM. Mmp13 expression was only high in IM and IC conditions (both contained ITS), 5-12 times at week 2 and 5-70 times at week 3 compared to BM condition respectively. Interestingly, Hif2a, Alp and Vegf expression was highest in IM condition at both week 2 and 3. Overall, in term of aggregate size, extracellular matrix stain for Alcian Blue and certain gene markers, IC is the most potent medium to induce extracellular matrix deposition and hypertrophic differentiation of MiTEC. Thus we chose IC medium to continue with in the next experiments. 


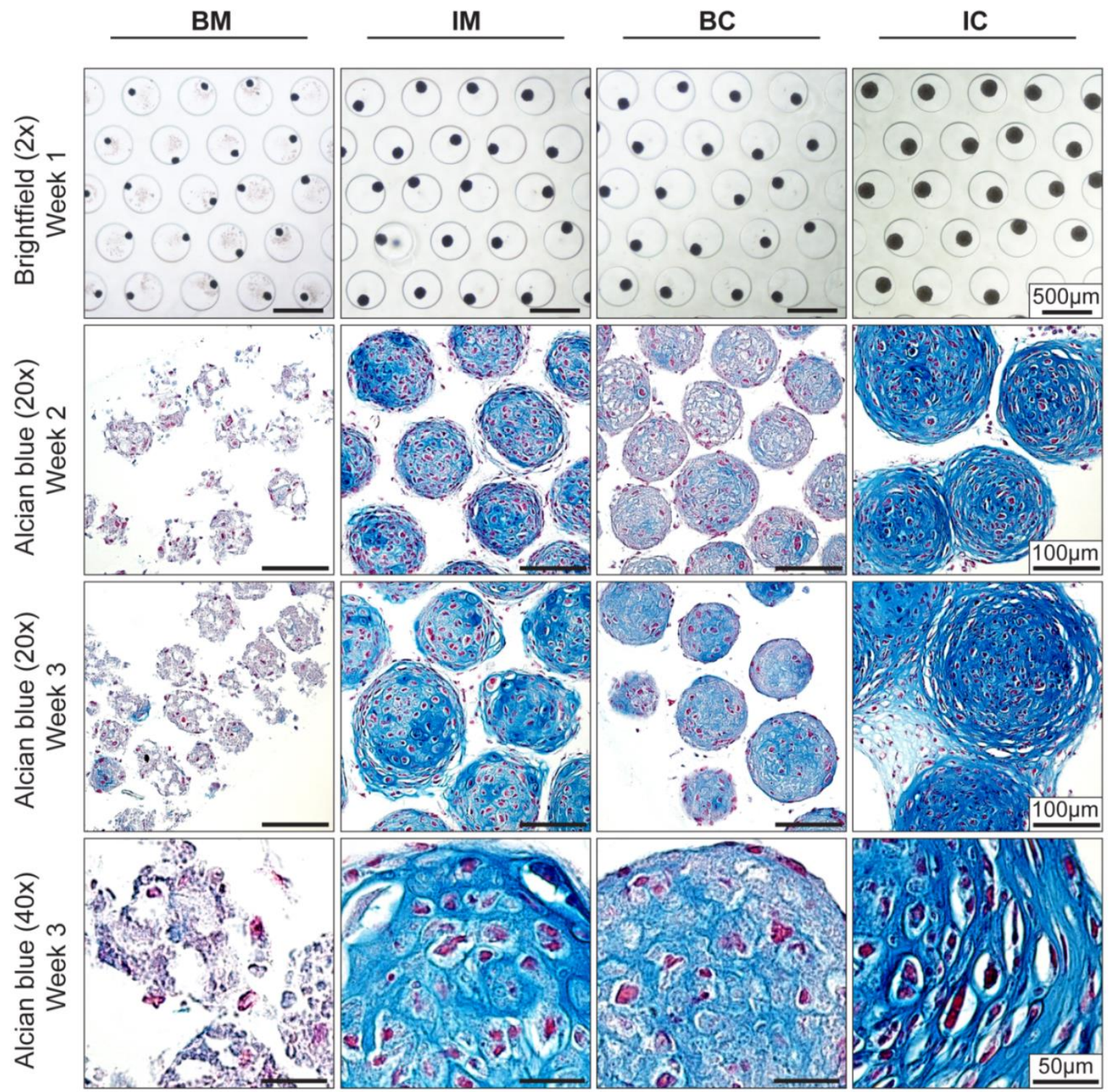

FIG 2. Effect of different media on MiTEC morphology. Aggregates were cultured for 3 weeks in different media (BM, IM, BC and IC). Brightfield images at week 1 (top row) show the difference in aggregate size in which the aggregates in IC medium was biggest. Alcian Blue staining at week 2 and 3 (row 2-4) shows maturation of the cartilage extracellular matrix; aggregates in IC medium were stained most intensely and uniformly. Hypertrophic cells can be found at both week 2 and week 3 in IM, BC and IC medium. 

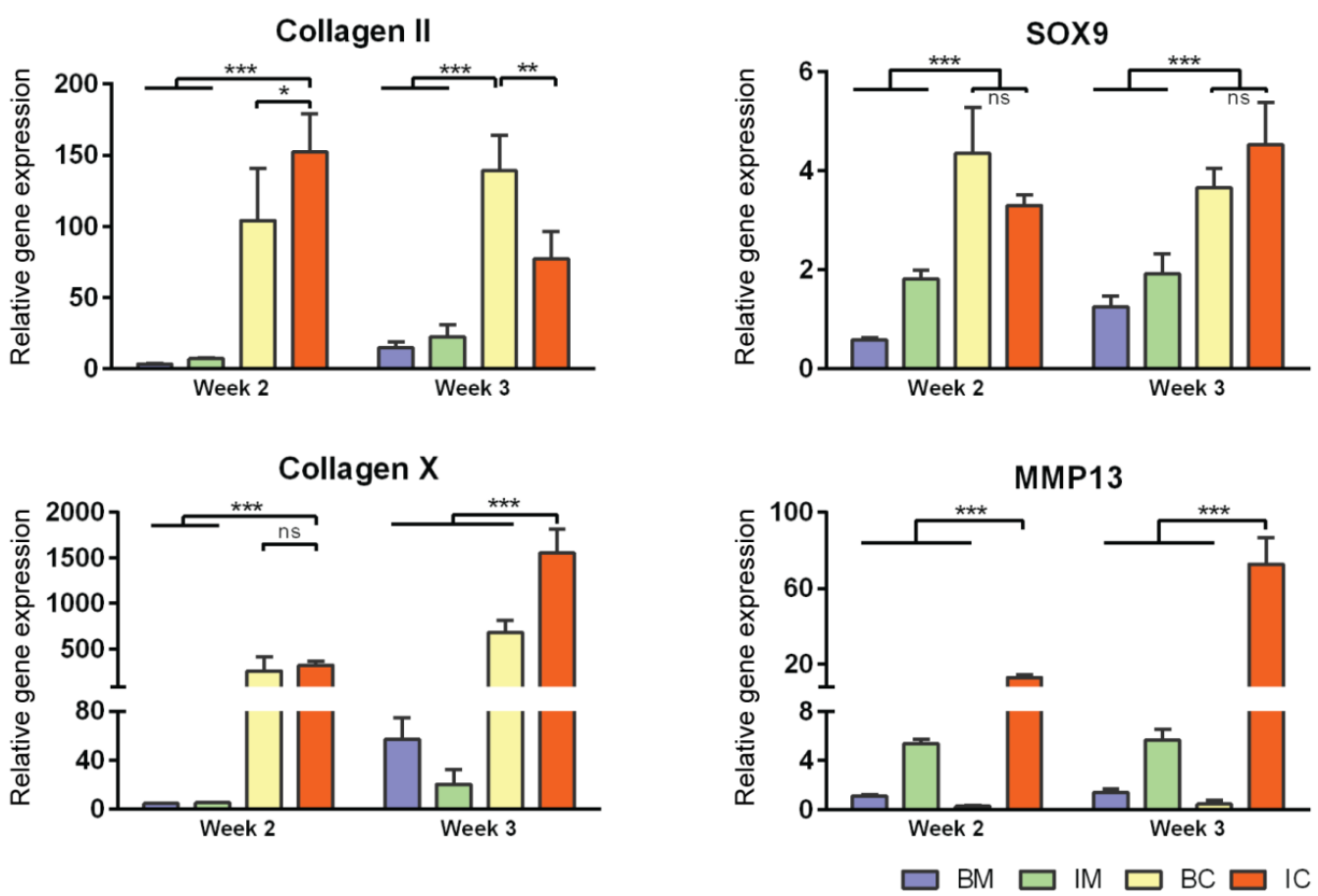

FIG 3. Effect of different media on MiTEC gene expression. Gene expression analysis of aggregates cultured in basic medium (BM - blue bar), BM + ITS (IM - green bar), BM + TGF $\beta$ + Dex (BC - yellow bar), and BM + ITS + TGF $\beta$ + Dex (IC - red bar) at week 2-3. Error bars represent standard deviation $(\mathrm{n}=3)$. $(*)$ denotes $\mathrm{p}<0.05,(* *)$ denotes $\mathrm{p}<0.01$, (***) denotes $\mathrm{p}<0.001$, and ns denotes non-significant.

\section{Induction of MiTEC mineralization with beta-glycerophosphate}

Just prior to endochondral ossification, end stage hypertrophic chondrocytes directs the formation of mineralized matrix, which becomes a scaffold for osteoprogenitor cells to interact with and secrete osteoid [31]. Pre-mineralizing the hypertrophic cartilage construct in vitro may improve and accelerate bone formation upon in vivo implantation. In this experiment, we induced in vitro mineralization of MiTECs with beta-glycerophosphate (bGP). MiTECs were cultured for 2 or 3 weeks in IC medium, followed by 1 week in mineralization medium with 10 $\mathrm{mM}$ bGP. MiTECs were collected at week 3 (2 weeks in IC medium plus 1 week in mineralization medium) and week 4 (3 weeks in IC medium plus 1 week in mineralization medium) for analysis. Calcium analysis showed a significant increase in the calcium content of MiTEC cultured in mineralization medium (Fig. 4A). Hydroxyproline content (estimated organic content of the extracellular matrix) increased from week 3-4 but was not affected by the bGP treatment as such. Histological analysis with Alizarin Red staining showed intense red nodules in the bGP condition (Fig. 4B). Gene expression analysis was performed for hypertrophic markers Hif2a, Alp, Vegf, collagen X and Mmp13 (Supp Fig. 2A). Interestingly, 
the addition of bGP increased Hif2 $\alpha$, Vegf, collagen type X and Mmp3 expression at week 4 but not week 3 of culture. Expression of Alp after 1 week in mineralization medium either decreased (week 3) or unchanged (week 4). Overall, in vitro mineralization of MiTEC could be efficiently achieved with bGP treatment, which may add an edge to the in vivo performance of the material.

A
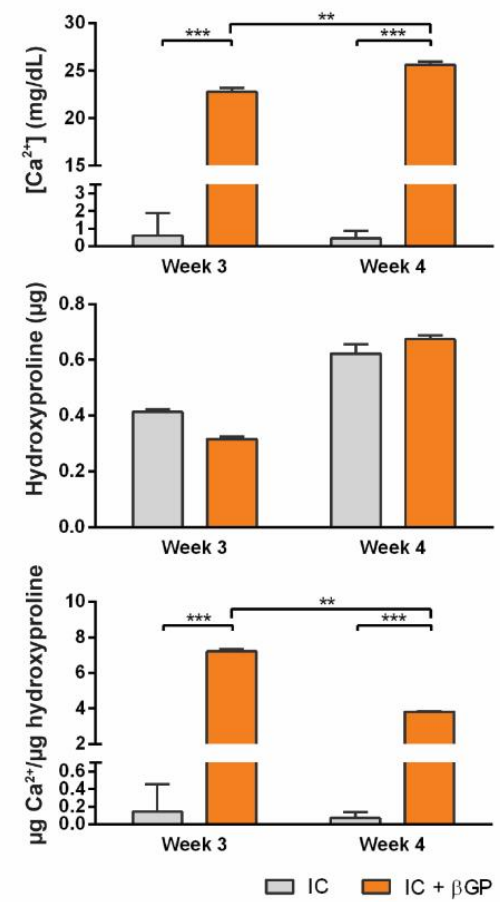

B
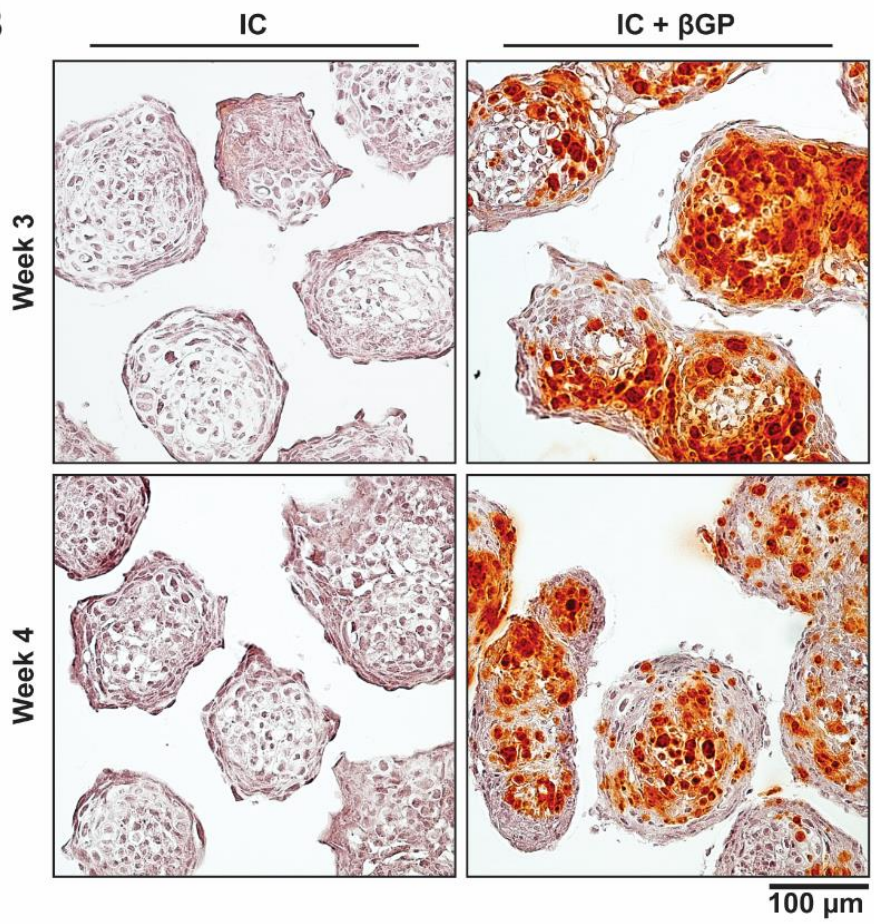

FIG. 4. Induction of MiTEC mineralization with beta-glycerophosphate. Calcium content, hydroxyproline content and calcium content per $\mu \mathrm{g}$ of hydroxyproline of aggregates cultured in IC medium for 2 or 3 weeks, plus 1 week treatment with bGP (A). Error bars represent standard deviation $(\mathrm{n}=3)$. $(* *)$ denotes $\mathrm{p}<0.01$, and $(* * *)$ denotes $\mathrm{p}<0.001$. Alizarin Red staining of aggregates cultured in IC medium for 2 or 3 weeks, plus 1 week treatment with bGP; red nodules can be seen homogeneously inside the aggregates $(\mathbf{B})$.

\section{Boosting Vegf secretion from MiTEC using the hypoxia mimetic phenanthroline}

Vascular invasion of the hypertrophic cartilage is a crucial event in endochondral bone formation and Vegf plays a critical role in it $[32,33]$. Enriching the extracellular matrix with Vefg may improve MiTEC performance. Previously, using high throughput screening, we identified phenanthroline as a hypoxia mimicking molecule capable of inducing Vegf expression and secretion in hMSCs [30]. Preliminary experiments were done to verify that phenanthroline can also stimulate Vegf expression and secretion of ATDC5 cells cultured in 
monolayer (data not shown). In aggregate culture, Vegf expression was highest in IM medium (Supp Fig. 1B), but Vegf secretion as detected in medium was highest in IC medium at about $2000 \mathrm{pg} / \mathrm{ml}$ (Fig. 5, top graph). To test the effect of phenanthroline on MiTEC, we cultured the aggregates for 2 or 3 weeks in IC medium, followed by a 2, 3 or 6 day exposure to $50 \mu \mathrm{M}$ phenanthroline. Phenanthroline induced a 7-fold induction of Vegf expression after a 2 day exposure at week 2 culture (Fig. 5, bottom graph). However, this expression reduced if the exposure was prolonged to 3 days and 6 days. Exposing MiTEC with phenanthroline for 2 days at week 3 of culture also resulted in an 8-fold increase in mRNA level. Vegf secretion baseline at $2000 \mathrm{pg} / \mathrm{ml}$ in IC medium was more than doubled when phenanthroline was added for 2 days at week 2 of culture (Fig. 5, middle graph). However, when phenanthroline treatment was extended to 6 days, secretion reduced to about $3000 \mathrm{pg} / \mathrm{ml}$. A 2-day stimulation in week 3 resulted in the same secretion level as in week 2. Thus, using phenanthroline, we could efficiently boost Vegf expression and secretion from MiTEC.
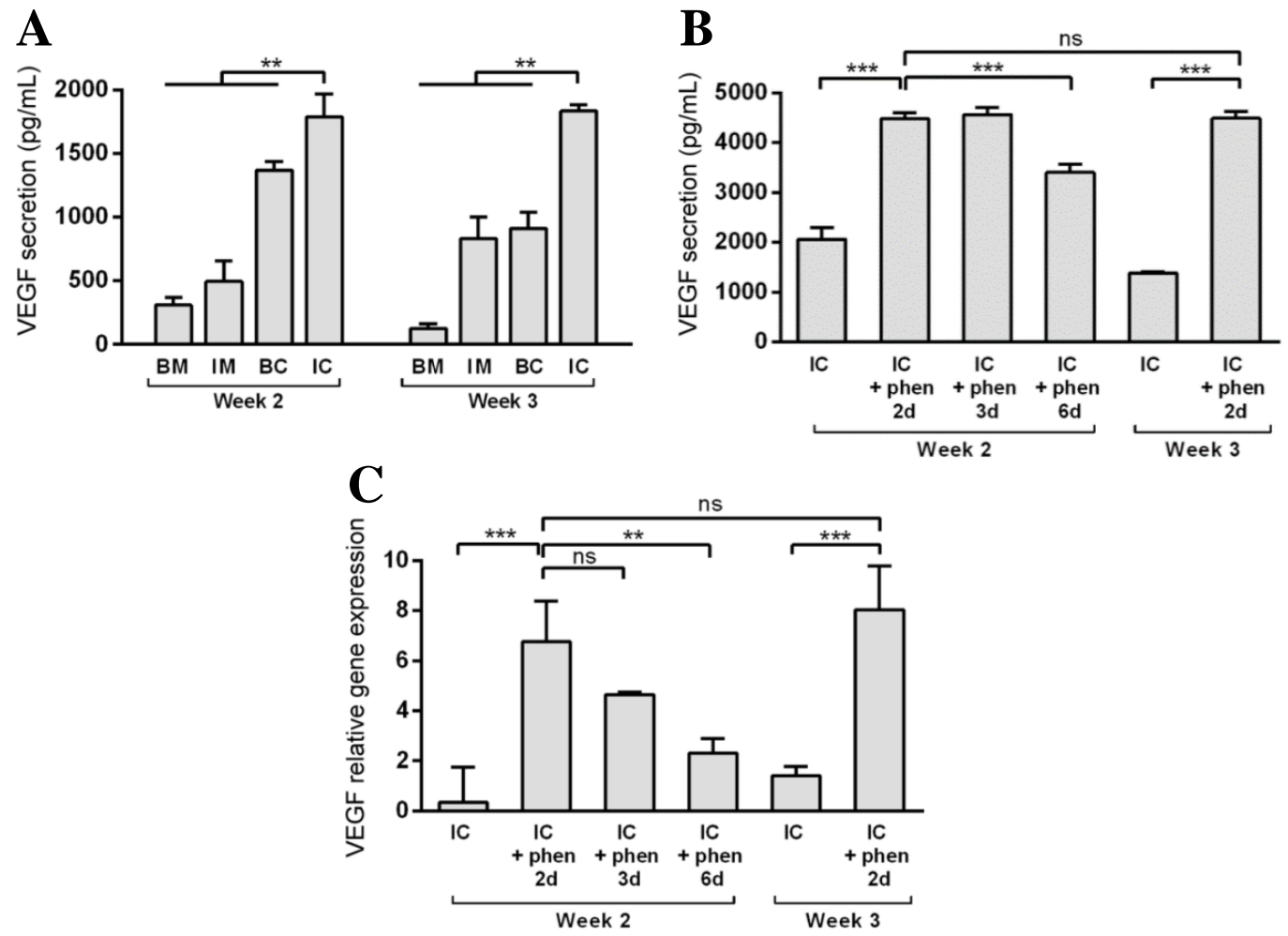

FIG. 5. Boosting Vegf expression and secretion from MiTEC using the hypoxia mimetic phenanthroline. VEGF secretion of aggregates cultured in BM, IM, BC and IC medium at week 2-3 (A). Vegf secretion (B) and expression (C) of aggregates cultured in IC medium for 2 or 3 weeks followed by phenanthroline treatment for 2, 3 or 6 days. Error bars represent standard deviation $(\mathrm{n}=3)$. $(* *)$ denotes $\mathrm{p}<0.01$, $(* * *)$ denotes $\mathrm{p}<0.001$, and $\mathrm{ns}$ denotes non-significant. 


\section{Devitalization and decellularization of MiTEC}

To make MiTEC an off-the-shelf product, devitalization/decellularization is necessary in order to reduce immunogenicity of the material. We devitalized MiTEC by liquid nitrogen (LN2) freeze-thawing and decellularized MiTEC by SDS processing following established protocols. Devitalized/ decellularized MiTECs were tested for cell survival by metabolism assay where no metabolic activity was found (data not shown). The amount of MiTECs harvested from one 12-well plate, decellularized with SDS and air dried is shown in Supp Fig. 2B. The average diameter of an aggregate after a 3-week culture in IC medium was $300 \mu \mathrm{m}$ (Fig. 6). Assuming each aggregate is a sphere; its volume is $4 / 3 \pi \times 150^{3}=14,137,167 \mu^{3}$ or $0.014 \mathrm{~mm}^{3}$. Each well of the 12-well plate containing 1585 aggregates yielded $22.4 \mathrm{~mm}^{3}$, and one 12-well plate yielded $268.9 \mathrm{~mm}^{3}$ of tissue. Four culture plates would be needed to make $1 \mathrm{CC}$ of tissue.

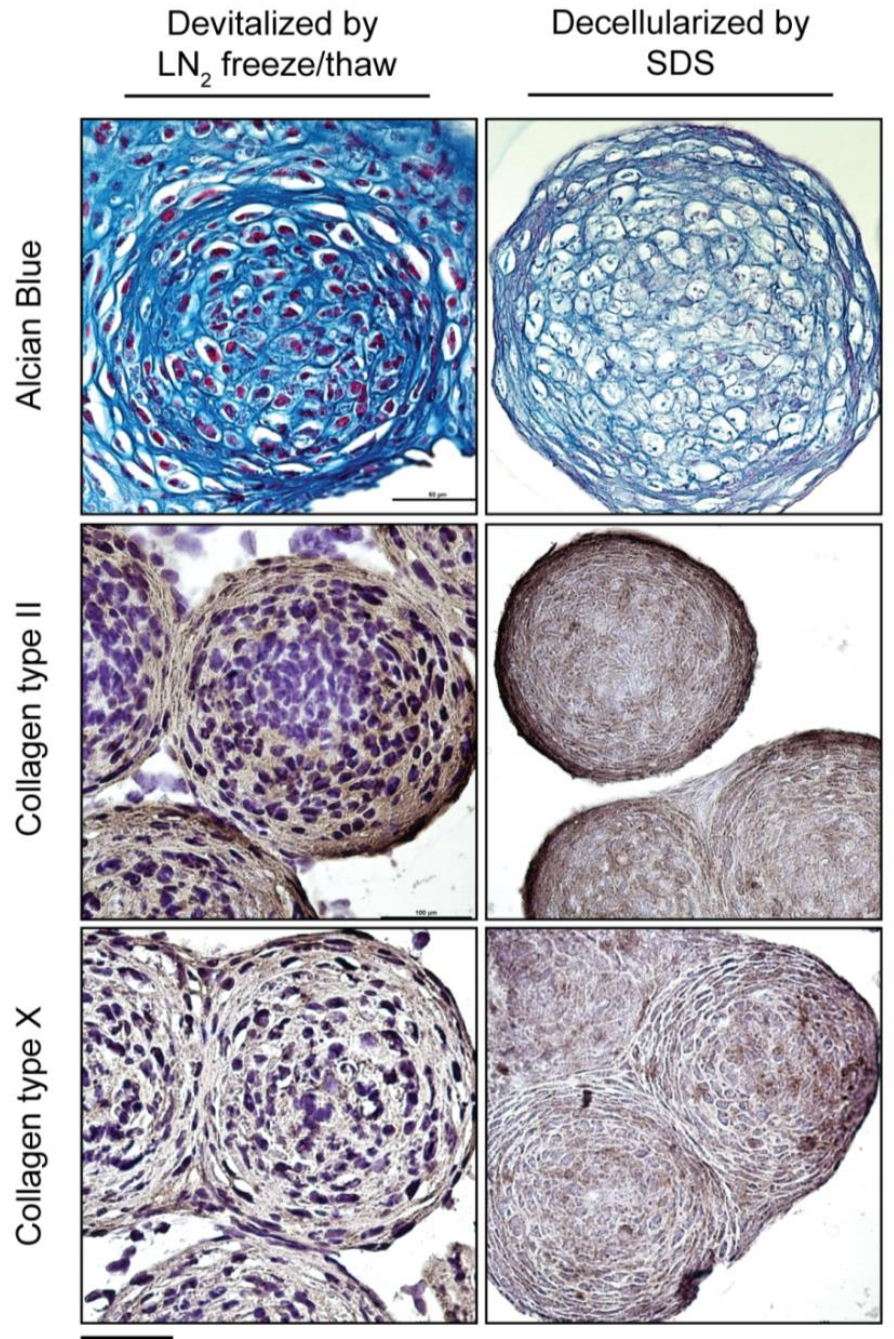

$\overline{50 \mu \mathrm{m}}$
Histological analysis with Alcian Blue and immunostaining for collagen type II and type $\mathrm{X}$ is shown in Fig. 6. No cell nuclei were visible in histological images of decellularized MiTEC. Alcian Blue staining was reduced significantly in the decellularized samples, however collagen type II and $\mathrm{X}$ staining were similar to that of the devitalized samples. Collagen type II staining was more eminent at the periphery of the aggregates, while collagen type $\mathrm{X}$ was distributed more uniformly.

FIG. 6. Devitalization and decellularization of MiTEC. Aggregates were cultured for 2 weeks in IC medium, then were either devitalized by repeated LN2 freeze/thaw or decellularized by SDS. Picture panel shows Alcian Blue staining (top row), collagen type II staining (middle row) and collagen type $\mathrm{X}$ staining (bottom row). 


\section{DCM influences chondrogenic differentiation of hMSCs}

To test the biological properties of our DCM, devitalized/decellularized MiTECs were mixed with hMSCs and pellet-cultured for 4 weeks in either basic medium or chondrogenic differentiation medium. Pellets of hMSCs mixed with LN2-devitalized MiTEC are referred to as LN2-pellet, pellets of hMSCs mixed with SDS-decellularized MiTEC as SDS-pellet. As a control, hMSCs were cultured in a pellet of 250,000 cells which is the same number of cells used in the mix-culture with MiTEC. After 4 weeks, the LN2-pellets and SDS-pellets cultured in chondrogenic medium developed a glassy appearance and felt very firm (Supp Fig. 2C). Gene expression analysis was performed for early chondrogenesis makers collagen type II, aggrecan and SOX9 (Fig. 7A). Relative to hMSC pellets, expression of these markers increased significantly in the SDS-pellet in chondrogenic medium. Even in basic medium, aggrecan expression went increase 20-fold in the SDS-pellet compared to hMSCs pellet. Expression of the 3 markers in the LN2-pellet was also higher than hMSC alone control, but lower than the SDS-pellets.

Alcian Blue staining shows the devitalized/decellularized MiTECs still visible and embedded inside the cell pellets (Fig. 7B). No cell is found inside the SDS treated MiTECs. In basic medium, beside the intense blue stain of the devitalized MiTEC aggregates, the rest of the pellet is weakly stained. In chondrogenic differentiation medium, the hMSCs alone control developed some blue stains only in some parts of the pellets. However, when hMSCs were cocultured with either LN2 or SDS treated MiTEC, Alcian Blue was intensely stained throughout the pellets. The MiTEC appeared to be shrunken inside the pellets under chondrogenic medium condition, with big gap between MiTEC and the human cells. With this experiment, we showed that devitalized/decellularized MiTEC has chondro-inductive potential in vitro.

\section{Discussion}

Although autologous bone are considered as the most superior grafting material for bone regeneration [34], other bone graft substitutes are increasingly preferred not only for the convenience of the surgeons but also for the good of the patients [35]. In nature, bone healing occurs through both the processes of endochondral and intramembranous ossification [36]. While intramembranous ossification is the fast route of building bone matrix by osteoprogenitor cells from adjacent bony tissue e.g. periosteum, it can only handle small and mechanically stable defect [36, 37]. The endochondral ossification route, although is slow and requires a lot more energy, is crucial for healing of large bone defect. After the initial forming of primary hematoma and fibrin-rich granulation tissue, endochondral formation starts with the deposition of cartilaginous matrix, called the soft callus. In animal models, the soft callus formation peaks at 7-9 days post trauma with a peak in collagen type II and proteoglycan such as aggrecan [38].

To date, no clinical case using hypertrophic cartilage for bone regeneration has been reported. Despite abundant proof-of-principle studies, no available method can yet produce

sufficient hypertrophic cartilage for clinical use. Here we demonstrated that using the cell line ATDC5 cultured in micro-aggregates, it was feasible to produce hypertrophic cartilage in unlimited amount. 
A

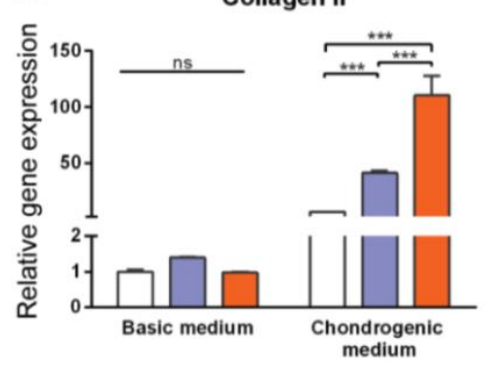

$\square$ hMSCs control pellet $\square$ LN2-pellet

B
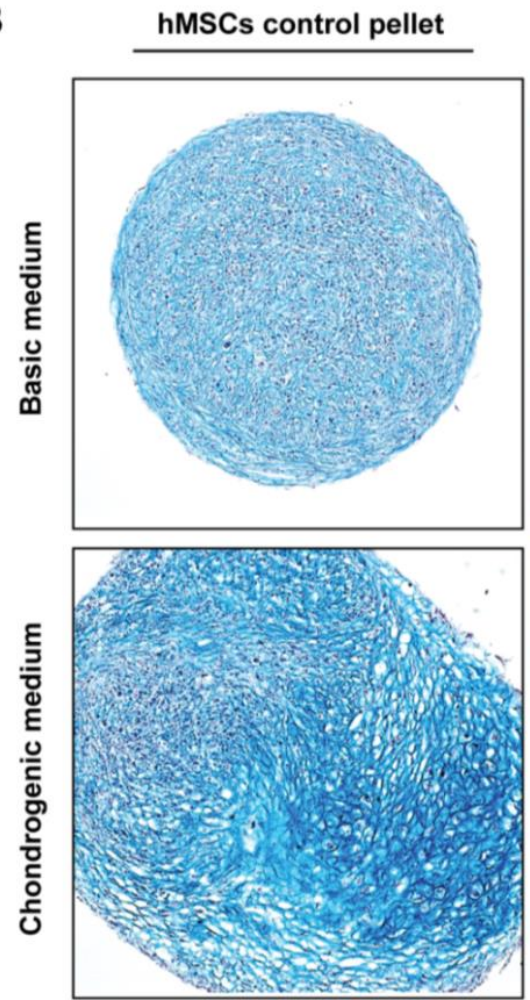

sox9

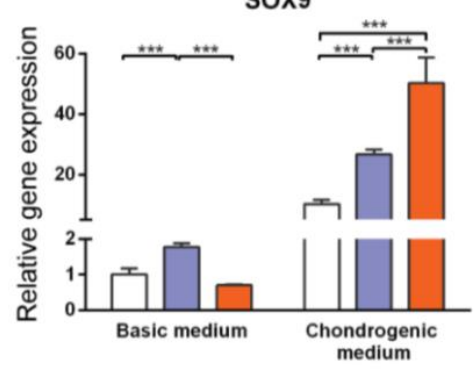

SDS-pellet
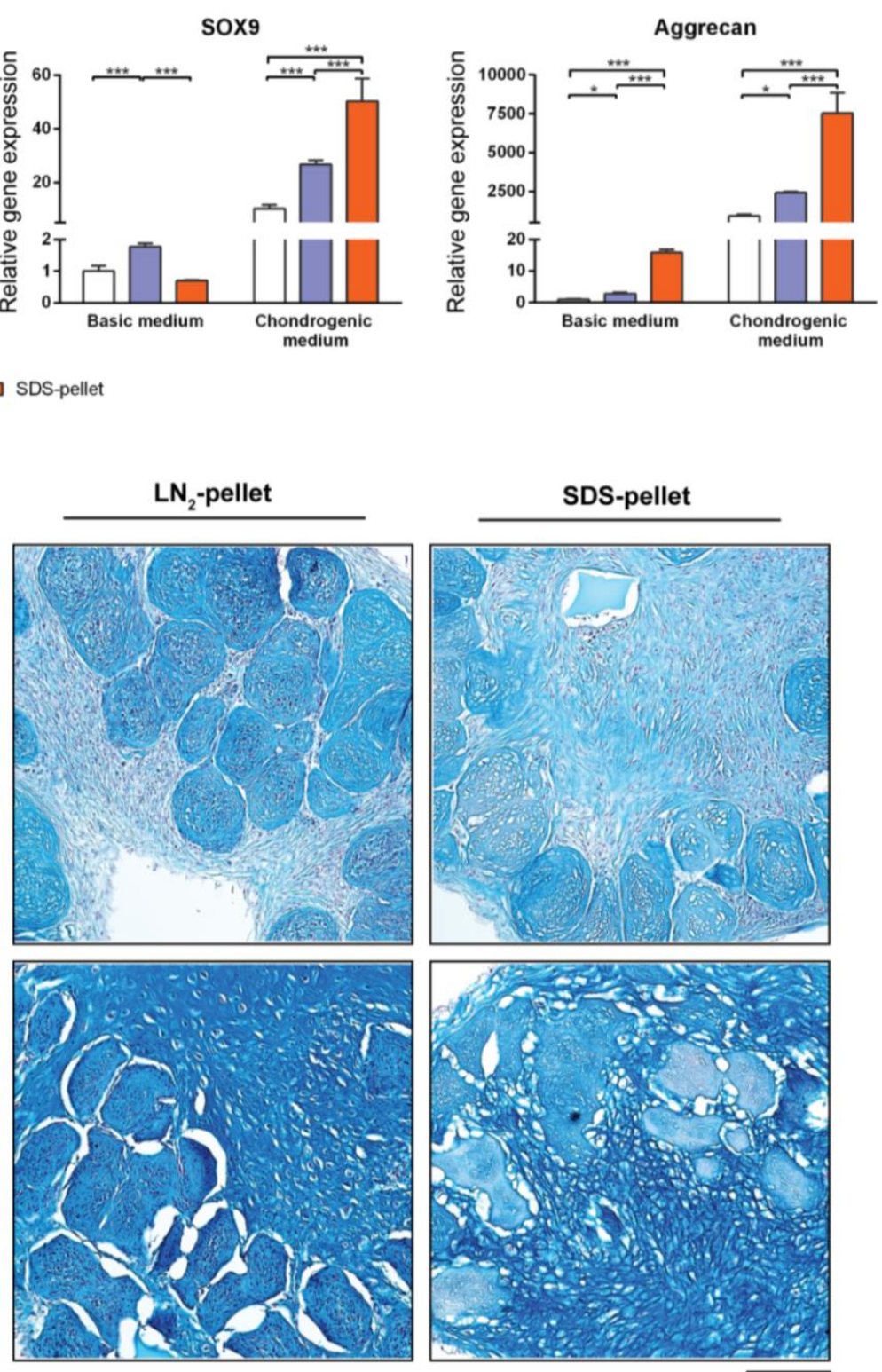

$\overline{200 \mu \mathrm{m}}$

FIG. 7. MiTEC affects chondrogenic differentiation of hMSCs. Gene expression analysis of hMSCs co-cultured with devitalized or decellularized MiTEC in basic medium or chondrogenic medium (A). Error bars represent standard deviation $(n=3)$. $(*)$ denotes $\mathrm{p}<0.05,(* *)$ denotes $\mathrm{p}<0.01,(* * *)$ denotes $\mathrm{p}<0.001$, and ns denotes non-significant. Alcian Blue staining of hMSCs co-cultured with devitalized or decellularized MiTEC in basic medium (top row) or chondrogenic medium (bottom row) (B). 
According to FDA's guideline for clinical issues concerning the use of xenotransplantation products in humans [39], "cell lines from animals may be established and used in the production of xenotransplantation products". Compared to primary cells, cell lines are easier to maintain and more consistent in quality. Since cell lines are carcinogenic, devitalization of the hypertrophic cartilage is necessary to reduce immunogenic concerns.

Whether or not the devitalized MiTEC is still capable of inducing endochondral ossification in vivo needs further experiments. Bourgine et al demonstrated that apoptosis-induced devitalized hypertrophic cartilage constructs could form bone in vivo while freeze-thawed devitalized constructs could not [18]. They attributed this to the significant loss of glycosaminoglycans, mineral content, and ECM-bound cytokines during the freeze-thaw cycles. We also observed a loss of glycosaminoglycans, as indicated by Alcian Blue staining, in the SDS decellularized MiTEC, but not in the freeze-thaw devitalized MiTEC. Apparently, the bioactivity of hypertrophic cartilage ECM may be lost by when a sub-optimal devitalization/decellularization process is used. Hence, the balance of removing immunogenicity and retaining osteoinductivity needs to be fine-tuned, for which many devitalization/decellularization methods have been reported [40-42]. Our model can potentially be adapted into a high throughput screening platforms in vitro and in vivo, allowing the devitalization/decellularization protocol to be empirically optimized.

Vascularization is a critical factor for successful integration of large grafts. Hypertrophic cartilage has an intrinsic property to produce angiogenic growth factors, which play a crucial role in endochondral ossification and many studies have shown the positive effect of adding VEGF in bone regeneration [33, 43]. It has been shown that lack of vascularization in the DCM constructs could be attributed to the loss of VEGF during the devitalization process [18]. Vascularization in the case of endochondral bone formation is essential for the recruitment of the host cells in order to remodel the cartilage template and subsequent ossification. We showed that stimulating MiTEC with the hypoxia mimicking molecule phenanthroline boosted VEGF secretion to double its basal level, although the VEGF content which remained in the decellularized MiTEC has not been analyzed. Since vascularization is paramount to the success of this model, it may be necessary to further optimize it for instance by combining it with current vascularization strategies, i.e. VEGF impregnation [44]. Similarly, pre-mineralization of MiTEC in vitro may be beneficial for bone formation in vivo. Calcium phosphate ceramics have a positive effect on osteogenesis [45] and even soluble calcium ions have a positive effect on osteogenic differentiation of hMSCs [46]. The degree of mineralization is another aspect which can be optimized for bone formation in vivo.

The inductive potential of decellularized ECM on stem cells $[47,48]$ has been reported. It was hypothesized that the acellular ECM of a tissue possessed the instructive cues that drive the differentiation of stem cells into this particular tissue type $[49,50]$. The instructive cues are the soluble factors and the macromolecules that regulate cell fate. To date, no study has demonstrated the inductive potential of hypertrophic cartilage matrix in vitro. In this paper, we show that devitalized/decellularized MiTEC, especially in combination with chondrogenic medium, enhanced chondrogenic differentiation of hMSCs in vitro. From histological images it appeared that the MiTEC ECM remained intact and hMSCs were not able to enter the cartilage 
matrix. Thus, it's arguable that the inductive potential of devitalized/decellularized MiTEC is caused by the soluble factors released from it, although the surface chemistry and topography of the tissue might also play a role. Interestingly, SDS decellularized MiTEC appeared to be more potent than the freeze-thaw devitalized aggregates. This may be similar to the biological activity of bone versus demineralized bone, where removal of bone mineral makes the osteogenic molecules available for interaction with the cells [51].

Unlike natural products harvested from cadavers where donor variation is inevitable, hypertrophic cartilage culture in vitro is a totally controllable process. There are many concerns over the variation in quality among allogenic bone grafts produced by different companies or even from different batches of product from the same company [52, 53]. Moreover, some products such as DBM and platelet gels containing "autologous growth factors" are not subjected to high level of regulatory scrutiny [54]. In contrast, MiTEC can be cultured and tested for both safety and efficacy following stringent GMP protocols. Here, we demonstrated how MiTEC can be manipulated in a number of ways to modify its extracellular matrix composition. The well plate format of this system allows for high throughput screening to be used in drug discovery and, using a different cell source or cell sources, one can even switch this model to the production of a totally different type of tissue, for instance to produce liver tissue or to study tumor cell biology. In conclusion, we have shown a method to produce clinically relevant-sized hypertrophic cartilage for bone regeneration by endochondral ossification. We decellularized the hypertrophic tissue and showed that the remaining ECM had inductive potential on chondrogenic differentiation of hMSCs in vitro. The logical next phase of this work is the pre-clinical evaluation of MiTEC in orthotopic models.

\section{Acknowledment}

We thank Prof. Dr. Marcel Karperien for providing the ATDC5 cell line, Dr. Nicolas Rivron and Erik Vrij for providing the stamps and PDMS molds for the spheroid culture system, and Dr. Ana Barradas for optimizing the micro-aggregate culture system. JdB and CvB acknowledge the financial support of the Dutch province of Limburg, and BLQ acknowledges the financial support of Pieken in de Delta Osst Nederland (PIDON) grant 91014. 


\section{Reference for Chapter 4 (MiTEC)}

1. Ferguson, C., et al., Does adult fracture repair recapitulate embryonic skeletal formation? Mech Dev, 1999. 87(1-2): p. 57-66.

2. Einhorn, T.A. and L.C. Gerstenfeld, Fracture healing: mechanisms and interventions. Nat Rev Rheumatol, 2014.

3. Gerstenfeld, L.C., et al., Fracture healing as a post-natal developmental process: molecular, spatial, and temporal aspects of its regulation. J Cell Biochem, 2003. 88(5): p. 873-84.

4. Durao, S.F., et al., Bone regeneration in osteoporotic conditions: healing of subcriticalsize calvarial defects in the ovariectomized rat. Int J Oral Maxillofac Implants, 2012. 27(6): p. 1400-8.

5. Dinopoulos, H., R. Dimitriou, and P.V. Giannoudis, Bone graft substitutes: What are the options? Surgeon, 2012. 10(4): p. 230-9.

6. Jukes, J.M., et al., Endochondral bone tissue engineering using embryonic stem cells. Proc Natl Acad Sci U S A, 2008. 105(19): p. 6840-5.

7. Scotti, C., et al., Recapitulation of endochondral bone formation using human adult mesenchymal stem cells as a paradigm for developmental engineering. Proc Natl Acad Sci U S A, 2010. 107(16): p. 7251-6.

8. Weiss, H.E., et al., A semi-autonomous model of endochondral ossification for developmental tissue engineering. Tissue Eng Part A, 2012. 18(13-14): p. 1334-43.

9. Farrell, E., et al., In-vivo generation of bone via endochondral ossification by in-vitro chondrogenic priming of adult human and rat mesenchymal stem cells. BMC Musculoskelet Disord, 2011. 12: p. 31.

10. Gawlitta, D., et al., Modulating endochondral ossification of multipotent stromal cells for bone regeneration. Tissue Eng Part B Rev, 2010. 16(4): p. 385-95.

11. Scotti, C., et al., Engineering of a functional bone organ through endochondral ossification. Proc Natl Acad Sci U S A, 2013. 110(10): p. 3997-4002.

12. Bahney, C.S., et al., Stem cell-derived endochondral cartilage stimulates bone healing by tissue transformation. J Bone Miner Res, 2014. 29(5): p. 1269-82.

13. Urist, M.R., Bone: formation by autoinduction. Science, 1965. 150(3698): p. 893-9.

14. Bridges, J.B. and J.J. Pritchard, Bone and cartilage induction in the rabbit. J Anat, 1958. 92(1): p. 28-38.

15. Urist, M.R. and L.F. Mc, Osteogenetic potency and new-bone formation by induction in transplants to the anterior chamber of the eye. J Bone Joint Surg Am, 1952. 34-A(2): p. 443-76.

16. Urist, M.R., T.H. Wallace, and T. Adams, The Function of Fibrocartilaginous Fracture Callus. Observations on Transplants Labelled with Tritiated Thymidine. J Bone Joint Surg Br, 1965. 47: p. 304-18.

17. Urist, M.R. and T. Adams, Cartilage or bone induction by articular cartilage. Observations with radioisotope labelling techniques. J Bone Joint Surg Br, 1968. 50(1): p. 198-215.

18. Bourgine, P.E., et al., Osteoinductivity of engineered cartilaginous templates devitalized by inducible apoptosis. Proc Natl Acad Sci U S A, 2014. 111(49): p. 17426-31.

19. Cunniffe, G.M., et al., Porous decellularized tissue engineered hypertrophic cartilage as a scaffold for large bone defect healing. Acta Biomater, 2015. 23: p. 82-90.

20. Jukes, J.M., et al., Critical Steps toward a tissue-engineered cartilage implant using embryonic stem cells. Tissue Eng Part A, 2008. 14(1): p. 135-47.

21. Siddappa, R., et al., Donor variation and loss of multipotency during in vitro expansion of human mesenchymal stem cells for bone tissue engineering. J Orthop Res, 2007. 25(8): p. 1029-41. 
22. Yao, Y. and Y. Wang, ATDC5: an excellent in vitro model cell line for skeletal development. J Cell Biochem, 2013. 114(6): p. 1223-9.

23. Altaf, F.M., et al., Ascorbate-enhanced chondrogenesis of ATDC5 cells. Eur Cell Mater, 2006. 12: p. 64-9; discussion 69-70.

24. Caron, M.M., et al., Hypertrophic differentiation during chondrogenic differentiation of progenitor cells is stimulated by BMP-2 but suppressed by BMP-7. Osteoarthritis Cartilage, 2013. 21(4): p. 604-13.

25. Rivron, N.C., et al., Tissue deformation spatially modulates VEGF signaling and angiogenesis. Proc Natl Acad Sci U S A, 2012. 109(18): p. 6886-91.

26. Fennema, E., et al., Spheroid culture as a tool for creating 3D complex tissues. Trends Biotechnol, 2013. 31(2): p. 108-15.

27. Alves, H., et al., Effect of antioxidant supplementation on the total yield, oxidative stress levels, and multipotency of bone marrow-derived human mesenchymal stromal cells. Tissue Eng Part A, 2013. 19(7-8): p. 928-37.

28. Mackay, A.M., et al., Chondrogenic differentiation of cultured human mesenchymal stem cells from marrow. Tissue Eng, 1998. 4(4): p. 415-28.

29. Kheir, E., et al., Development and characterization of an acellular porcine cartilage bone matrix for use in tissue engineering. J Biomed Mater Res A, 2011. 99(2): p. 283-94.

30. Doorn, J., et al., A small molecule approach to engineering vascularized tissue. Biomaterials, 2013. 34(12): p. 3053-63.

31. Kronenberg, H.M., Developmental regulation of the growth plate. Nature, 2003. 423(6937): p. 332-6.

32. Dai, J. and A.B. Rabie, VEGF: an essential mediator of both angiogenesis and endochondral ossification. J Dent Res, 2007. 86(10): p. 937-50.

33. Yang, Y.Q., et al., The role of vascular endothelial growth factor in ossification. Int J Oral Sci, 2012. 4(2): p. 64-8.

34. Pape, H.C., A. Evans, and P. Kobbe, Autologous bone graft: properties and techniques. J Orthop Trauma, 2010. 24 Suppl 1: p. S36-40.

35. Parikh, S.N., Bone graft substitutes: past, present, future. J Postgrad Med, 2002. 48(2): p. 142-8.

36. Marsell, R. and T.A. Einhorn, The biology of fracture healing. Injury, 2011. 42(6): p. 5515 .

37. Shapiro, F., Bone development and its relation to fracture repair. The role of mesenchymal osteoblasts and surface osteoblasts. Eur Cell Mater, 2008. 15: p. 53-76.

38. Einhorn, T.A., The cell and molecular biology of fracture healing. Clin Orthop Relat Res, 1998(355 Suppl): p. S7-21.

39. Food, U. and D. Administration, Guidance for Industry: Source Animal, Product, Preclinical, and Clinical Issues Concerning the Use of Xenotransplantation Products in Humans. 2003, 2011.

40. Lu, H., et al., Comparison of decellularization techniques for preparation of extracellular matrix scaffolds derived from three-dimensional cell culture. J Biomed Mater Res A, 2012. 100(9): p. 2507-16.

41. Crapo, P.M., T.W. Gilbert, and S.F. Badylak, An overview of tissue and whole organ decellularization processes. Biomaterials, 2011. 32(12): p. 3233-43.

42. Dong, J., et al., [Recent research progress of decellularization of native tissues]. Sheng Wu Yi Xue Gong Cheng Xue Za Zhi, 2012. 29(5): p. 1007-13.

43. Aryal, R., et al., Bone morphogenetic protein-2 and vascular endothelial growth factor in bone tissue regeneration: new insight and perspectives. Orthop Surg, 2014. 6(3): p. 171-8.

44. Krishnan, L., N.J. Willett, and R.E. Guldberg, Vascularization strategies for bone regeneration. Ann Biomed Eng, 2014. 42(2): p. 432-44. 
45. Zhang, J., et al., The size of surface microstructures as an osteogenic factor in calcium phosphate ceramics. Acta Biomater, 2014. 10(7): p. 3254-63.

46. Barradas, A.M., et al., A calcium-induced signaling cascade leading to osteogenic differentiation of human bone marrow-derived mesenchymal stromal cells. Biomaterials, 2012. 33(11): p. 3205-15.

47. Hoganson, D.M., et al., Differentiation of human bone marrow mesenchymal stem cells on decellularized extracellular matrix materials. J Biomed Mater Res A, 2014. 102(8): p. 2875-83.

48. Guo, Y., et al., Proliferative effect and osteoinductive potential of extracellular matrix coated on cell culture plates. Springerplus, 2013. 2(1): p. 303.

49. Brown, B.N. and S.F. Badylak, Extracellular matrix as an inductive scaffold for functional tissue reconstruction. Transl Res, 2014. 163(4): p. 268-85.

50. Reilly, G.C. and A.J. Engler, Intrinsic extracellular matrix properties regulate stem cell differentiation. J Biomech, 2010. 43(1): p. 55-62.

51. Zhang, M., R.M. Powers, Jr., and L. Wolfinbarger, Jr., A quantitative assessment of osteoinductivity of human demineralized bone matrix. J Periodontol, 1997. 68(11): p. 1076-84.

52. Aghdasi, B., et al., A review of demineralized bone matrices for spinal fusion: the evidence for efficacy. Surgeon, 2013. 11(1): p. 39-48.

53. Bae, H.W., et al., Intervariability and intravariability of bone morphogenetic proteins in commercially available demineralized bone matrix products. Spine (Phila Pa 1976), 2006. 31(12): p. 1299-306; discussion 1307-8.

54. Greenwald, A.S., et al., Bone-graft substitutes: facts, fictions, and applications. J Bone Joint Surg Am, 2001. 83-A Suppl 2 Pt 2: p. 98-103. 
Supp Table 1. Primer sequence

\begin{tabular}{|c|c|}
\hline Name & Primer sequence \\
\hline \multirow[t]{2}{*}{ B2m: beta-2 microglobulin [mouse] } & 5'-CATGGCTCGCTCGGTGACC-3' \\
\hline & 5'- AATGTGAGGCGGGTGGAACTG-3' \\
\hline \multirow[t]{2}{*}{ Col2a1: collagen type II alpha 1 [mouse] } & 5'-CAAGGCCCCCGAGGTGACAAA-3' \\
\hline & 5'-GGGGCCAGGGATTCCATTAGAGC-3' \\
\hline \multirow[t]{2}{*}{ Col10a1: collagen type $\mathrm{X}$ alpha 1 [mouse] } & 5'-CATAAAGGGCCCACTTGCTA-3' \\
\hline & 5'-TGGCTGATATTCCTGGTGGT-3' \\
\hline \multirow[t]{2}{*}{ Acan: aggrecan [mouse] } & 5'-AGAACCTTCGCTCCAATGACTC-3' \\
\hline & 5'-AGGGTGTAGCGTGTGGAAATAG-3' \\
\hline \multirow[t]{2}{*}{ Sox9: SRY-box9 [mouse] } & 5'-CCACGGAACAGACTCACATCTCTC-3' \\
\hline & 5'-CTGCTCAGTTCACCGATGTCCACG-3' \\
\hline \multirow[t]{2}{*}{ Hif1a: hypoxia inducible factor 1 alpha [mouse] } & 5'- TGCTCATCAGTTGCCACTTC-3' \\
\hline & 5'- TGGGCCATTTCTGTGTGTAA-3' \\
\hline \multirow[t]{2}{*}{ Hif2a: hypoxia inducible factor 2 alpha [mouse] } & 5'- TGAGTTGGCTCATGAGTTGC-3' \\
\hline & 5'- CTCACGGATCTCCTCATGGT-3' \\
\hline \multirow[t]{2}{*}{ Alp: alkaline phosphatase [mouse] } & 5'- AACCCAGACACAAGCATTCC-3' \\
\hline & 5'- GAGACATTTTCCCGTTCACC-3' \\
\hline \multirow[t]{2}{*}{ Mmp13: matrix metallopeptidase 13 [mouse] } & 5'- AGGCCTTCAGAAAAGCCTTC-3' \\
\hline & 5'- TCCTTGGAGTGATCCAGACC-3' \\
\hline \multirow[t]{2}{*}{ B2M: beta-2 microglobulin [human] } & 5' GACTTGTCTTTCAGCAAGGA 3' \\
\hline & 5' ACAAAGTCACATGGTTCACA 3' \\
\hline \multirow[t]{2}{*}{ COL2A: collagen type II alpha [human] } & 5' CGTCCAGATGACCTTCCTACG 3' \\
\hline & 5' TGAGCAGGGCCTTCTTGAG 3' \\
\hline \multirow[t]{2}{*}{ ACAN: aggrecan [human] } & 5' AGAATCCACCACCACCAG 3' \\
\hline & 5' ATGCTGGTGCTGATGACA 3' \\
\hline \multirow[t]{2}{*}{ SOX9: SRY-box9 [human] } & 5' TGGGCAAGCTCTGGAGACTTC 3' \\
\hline & 5' ATCCGGGTGGTCCTTCTTGTG 3' \\
\hline
\end{tabular}


A

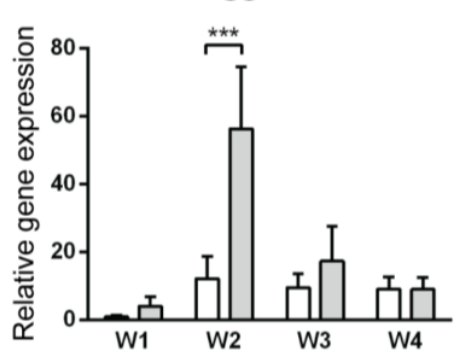

ALP

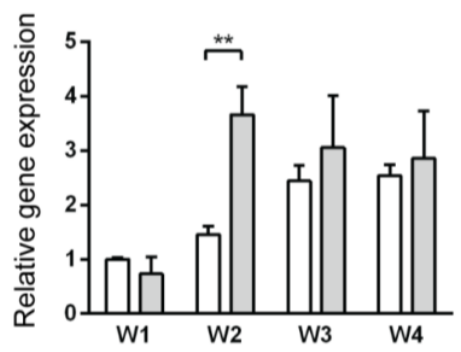

HIF1 $\alpha$

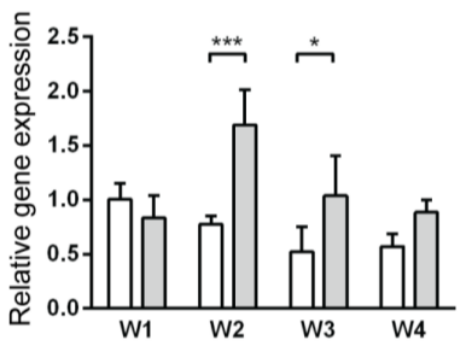

VEGF $\alpha$
HIF2 $\alpha$

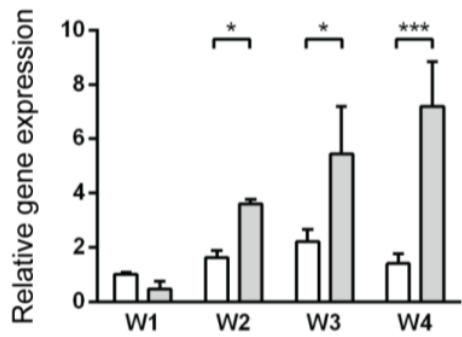

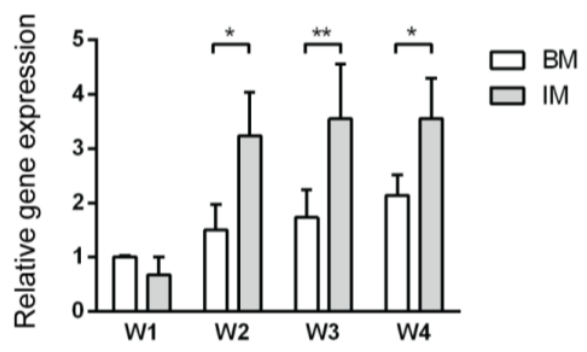

B
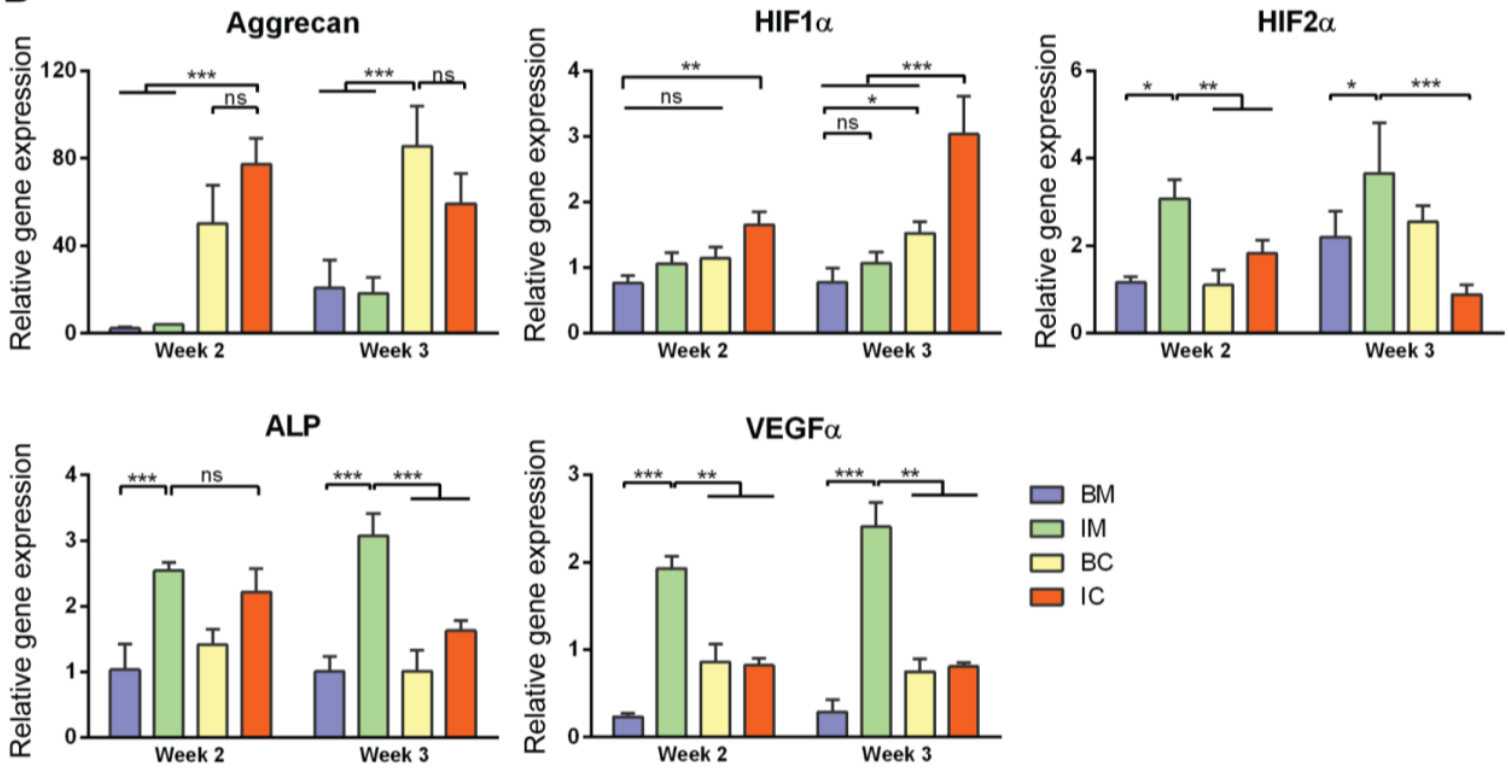

Supp Fig. 1. Gene expression of MiTEC cultured in BM (white bar) and IM (gray bar) at week 1-4 (A). Gene expression of MiTEC cultured in BM (blue bar), IM (green bar), BC (yellow bar) and IC (red bar) medium at week 2-3 (B). Error bars represent standard deviation $(\mathrm{n}=3) .(*)$ denotes $\mathrm{p}<0.05,(* *)$ denotes $\mathrm{p}<0.01,(* * *)$ denotes $\mathrm{p}<0.001$, and ns denotes non-significant. 

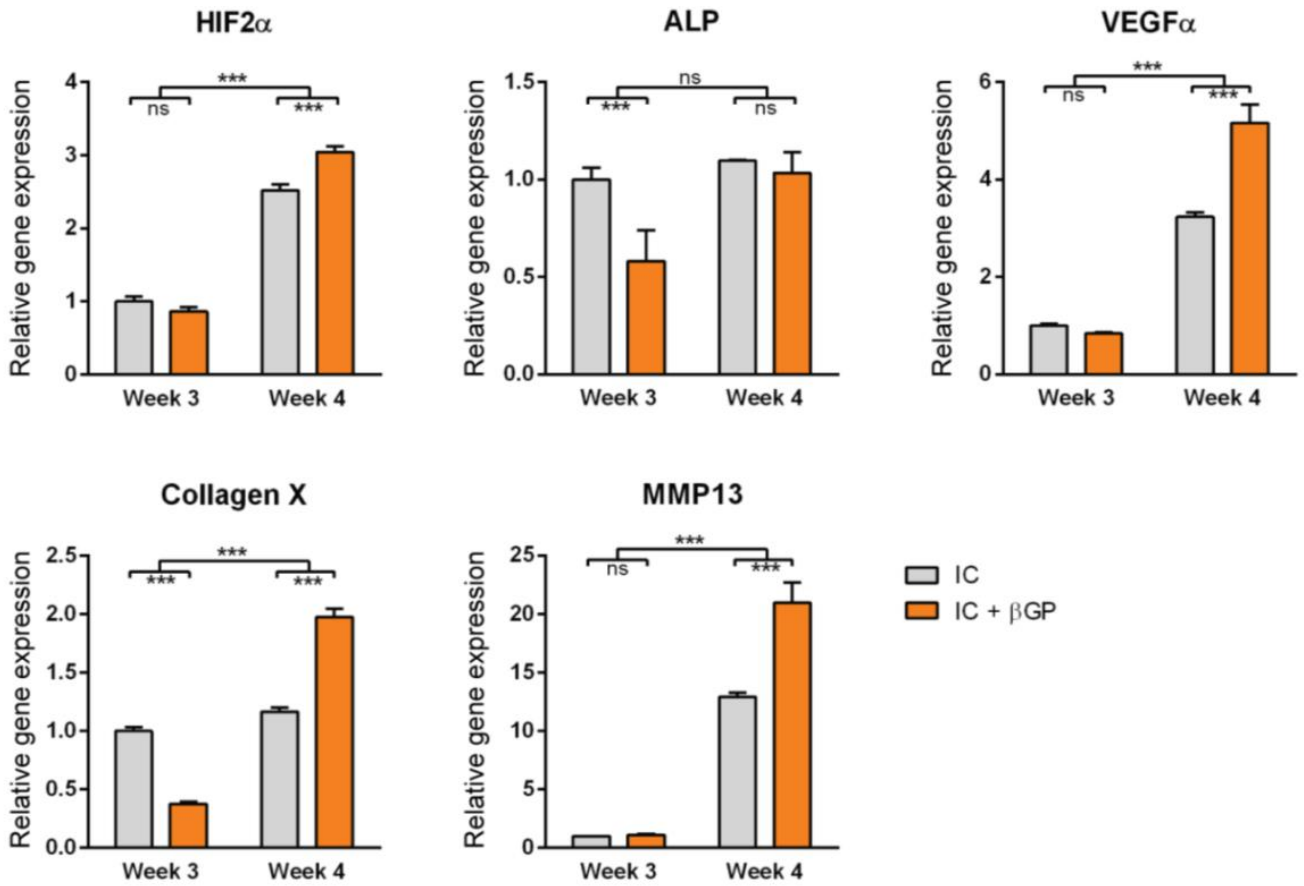

B

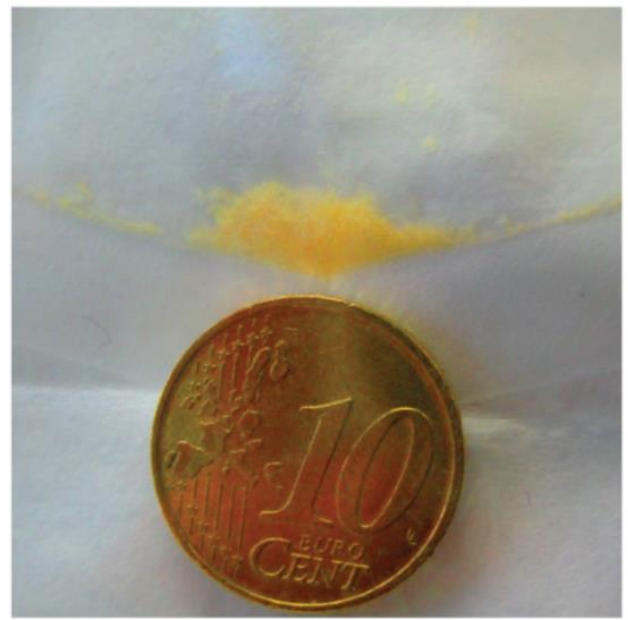

C

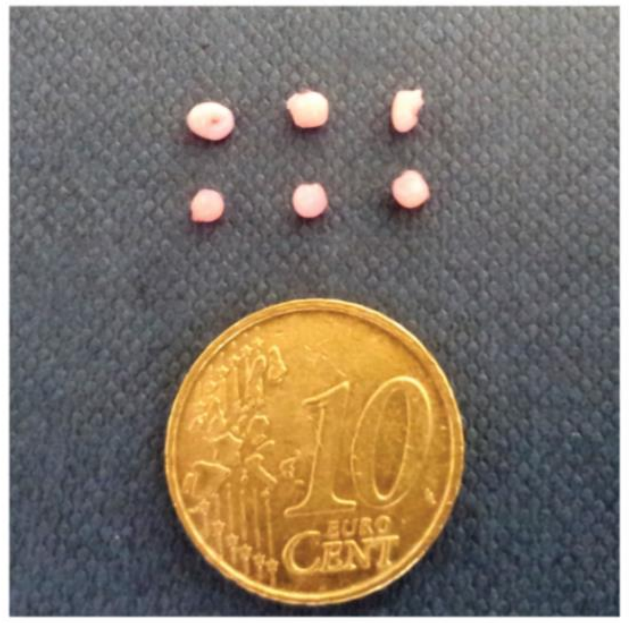

Supp Fig. 2. Gene expression analysis of MiTEC cultured in basic medium and mineralization medium (1 week in mineralization medium before time point) at week 3 and 4 (A). Error bars represent standard deviation $(\mathrm{n}=3) .(*)$ denotes $\mathrm{p}<0.05,(* *)$ denotes $\mathrm{p}<0.01,(* * *)$ denotes $\mathrm{p}<0.001$, and ns denotes nonsignificant. MiTEC were cultured in IC medium for 2 weeks, decellularized with SDS and air-dried; the picture showed the amount of MiTEC harvested from one 12-well plate (B). Pellets of 2.5x105 hMSCs mixed with devitalized (top row) and decellularized (bottom row) MiTEC, and cultured in chondrogenic medium for 4 weeks (C). 


\title{
Chapter 5
}

\section{Micro-Topographies Promote Late Chondrogenic Differentiation Markers in the ATDC5 Cell Line}

\author{
Bach Q. Le, Aliaksei Vasilevich, Steven Vermeulen, Frits Hulshof, \\ Dimitrios F. Stamatialis, Clemens van Blitterswijk, and Jan de Boer
}

\begin{abstract}
$\mathrm{C}$ hemical and mechanical cues are well-established influencers of in vitro chondrogenic differentiation of ATDC5 cells. Here, we investigate the role of topographical cues in this differentiation process, a study not been explored before. Previously, using a library of surface micro-topographies we found some distinct patterns that induced alkaline phosphatase (ALP) production in human mesenchymal stromal cells. ALP is also a marker for hypertrophy, the end stage of chondrogenic differentiation preceding bone formation. Thus, we hypothesized that these patterns could influence end-stage chondrogenic differentiation of ATDC5 cells. In this study, we randomly selected seven topographies among the ALP influencing hits. Cells grown on these surfaces displayed varying nuclear shape and actin filament structure. When stimulated with insulin-transferrin-selenium (ITS) medium, nodule formation occurred and in some cases showed alignment to the topographical patterns. Gene expression analysis of cells growing on topographical surfaces in the presence of ITS medium revealed a downregulation of early markers and upregulation of late markers of chondrogenic differentiation compared to cells grown on a flat surface. In conclusion, we demonstrated that surface topography in addition to other cues can promote hypertrophic differentiation suitable for bone tissue engineering.
\end{abstract}




\section{Introduction}

Surface micro/nano-topography is increasingly recognized as an important element in the design of medical implants to obtain the intended functionality. Cell-material interaction is not only influenced by chemistry, but is also dependent on the physical events occurring at the cellmaterial interface. Various studies have shown the effect of surface topography on cell fate decision in vitro [1-4]. Many attempts have been made to modify implant surface for better biocompatibility and integration of the host tissue [5-7].

Surface topography is no stranger in the field of bone tissue engineering. As mentioned in the review of Wennerberg and Albrektsson [8], as early as the beginning of the 1980s, surface structure was identified as one of the factors particularly important for the incorporation of implants into bone. Rougher surfaces had a more beneficial effect for osteointegration over smooth surfaces, due to the larger surface area of the former [9]. Later, advances in micro and nanotechnology enabled researchers to create precision-designed surface topography, starting a new field of material surface topography. Dalby et al. used electron beam lithography to fabricate nanoscale topographical surfaces featuring 120-nm-diameter, 100-nm deep nanopits placing from highly ordered symmetries to somewhat random placements [2]. Interestingly, they discovered that the random-placed nanopit topography stimulated human mesenchymal stem cells (hMSCs) to produce bone mineral in vitro without any osteogenic supplement. Although not specifically biomimetic, as declared by the authors, this approach is clearly useful in the regenerative medicine framework. To expand the effort in elucidating the instructive power of surface topography, we previously created a topography screening platform, the TopoChip, consisting of 2176 distinct surface topographies on a $2 \times 2 \mathrm{~cm}$ chip. In one TopoChip screen, we identified topographies that induce alkaline phosphatase (ALP) production from hMSCs [10]. Since ALP is also a marker for hypertrophic differentiation of chondrocytes [11], we hypothesized that these topographies could influence the different stages of chondrocyte differentiation.

Previously, we have successfully tissue-engineered hypertrophic cartilage in vitro using embryonic stem cells [12]. However, because of ethical controversy and the potential hazard of teratoma formation, we are interested in further exploring different methods of manipulating chondrocyte differentiation and extracellular matrix (ECM) deposition. In preceding studies, we used the ATDC5 chondroprogenitor cell line, an in vitro model system that has been used extensively in cartilage and bone research [13]. Our goal is to aid the field of bone tissue engineering by utilizing ATDC5 as a workhorse to produce hypertrophic cartilage matrix in vitro, which can be used in vivo with/without decellularization for endochondral ossification. To increase yield, we performed a screen of the LOPAC-1280 drug library and discovered tetradecylthioacetic acid as a drug that enhances collagen production by ATDC5 [14]. In another approach, we employed a micro-well platform to culture ATDC5 in small aggregates of 1000 cells, resulting in a better and more homogeneous cartilage matrix than cells growing on common tissue culture-treated plates (chapter 3).

During the development of an ATDC5 monolayer on a flat surface, when exposed to ITS medium, spots of cell aggregates appear randomly across the surface due to hyperplasia. This is 
concomitant with a series of differentiation events characterized by an increase in expression of chondrogenic markers. The ATDC5 cell line is extensively used to study the effect of microenvironment modifications on this chondrogenic differentiation process. Examples include the use of growth factors [15], ascorbic acid [16], oxygen tension [17] and substrate stiffness [18]. In this study, we further extend these different approaches by studying the effect of surface topographies during the chondrogenesis of ATDC5 cells. Surface micro-topography has been known to affect cell shape, spreading [19] and differentiation [20]. But whether surface microtopography can affect the chondrogenic differentiation process is unknown. Instead of using the full TopoChip, we opted to select seven topographies from the hits of our previous TopoChip screen. These hits had a profound positive and negative effect on ALP secretion of hMSCs (article submitted). The seven topographies were fabricated on discs with a diameter of $1.5 \mathrm{~cm}$, allowing the use of traditional molecular biology techniques such as quantitative polymerase chain reaction. We wanted to explore the relationship between cell/nuclei shape and gene expression profile of chondrogenesis-related genes. Ultimately, we asked what particular surface topography would induce a specific cell morphology, which in turn would influence the pathway to hypertrophic differentiation of ATDC5 cells.

\section{Materials and Methods}

\section{Cell culture}

ATDC5 cells were obtained from RIKEN cell bank (Japan). Basic medium (BM) for ATDC5 consisted of DMEM/F-12 GlutaMAX (Life Technologies), 5\% v/v fetal bovine serum (Lonza), $0.2 \mathrm{mM} \mathrm{l-ascorbic} \mathrm{acid-2} \mathrm{phosphate,} 100 \mathrm{U} / \mathrm{mL}$ of penicillin, and $100 \mathrm{mg} / \mathrm{mL}$ of streptomycin. To stimulate chondrogenic differentiation and nodule formation, an insulintransferrin-selenium (ITS) solution (100x; Life Technologies) was supplemented to BM. Cells were grown at $37^{\circ} \mathrm{C}$ in a humid atmosphere with $5 \% \mathrm{CO}_{2}$. Medium was refreshed two to three times per week and cells were used for further subculturing or cryopreservation upon reaching near confluence.

\section{Producing large topographical surfaces}

Topographies with a feature height of $10 \mathrm{~mm}$ were produced on polystyrene (PS) by hot embossing of a PS film (Goodfellow). The inverse structure of the topographies was produced on silicon by standard photolithography and deep reactive etching as described before [10]. The silicon imprint was used to make a positive mould on polydimethylsiloxane (PDMS). The PDMS mould was required to create a second negative mould in OrmoStamp hybrid polymer (micro resist technology Gmbh), which serves as the mould for hot embossing the PS films. The fabrication of the OrmoStamp mould was necessary due to the fact that demoulding of PS from silicon often led to destruction of the mould. The hot embossing conditions were $140^{\circ} \mathrm{C}$ for 5 min at a pressure of $10 \mathrm{Bar}$, with a demoulding temperature of $90^{\circ} \mathrm{C}$. Before cell culture, the PS topographies were briefly treated with oxygen plasma to improve cell adhesion. Plasma treatment was performed for $30 \mathrm{~s}$ at $75 \mathrm{mTor}, 50 \mathrm{sccm} \mathrm{O}_{2}$, and $50 \mathrm{~W}$. Topographical numbering 
is based on our TopoUnit database, a repository containing the design parameters for each individual topography.

\section{DAPI and Phalloidin staining}

Before any experiment, all topographical surfaces and flat control were pre-wetted in BM for $48 \mathrm{~h}$ at $37^{\circ} \mathrm{C}$. ATDC5 were seeded at a density of $2 \times 10^{4}$ cells per $\mathrm{cm}^{2}$ in BM on the different surfaces. After $24 \mathrm{~h}$, the surfaces were washed briefly with phosphate-buffered saline (PBS) and then fixed in $4 \%$ paraformaldehyde (Merck) in PBS for $10 \mathrm{~min}$. This was followed by a permeabilization step with $0.1 \%$ Triton X-100 (Sigma-Aldrich) in PBS for $10 \mathrm{~min}$, and then blocking in 5\% BSA (Sigma-Aldrich) for $30 \mathrm{~min}$. After that, the surfaces were incubated for $1 \mathrm{~h}$ in the dark with Alexa Fluor 594 Phalloidin (1:200; Life Technologies) containing 0.03\% Triton X-100 in PBS. After washing with PBS ( 3 x $10 \mathrm{~min})$, the surfaces were incubated with DAPI (1:100 in PBS; Life Technologies) for 10 min in the dark. After another wash with PBS, the surfaces were mounted on glass slides with Mowiol (Sigma-Aldrich) and left to dry overnight in the dark at room temperature.

\section{Image acquisition and analysis}

Fixed samples were inverted and fluorescent images were acquired through the glass coverslip using a BD pathway microscope and analyzed with CellProfiler [21]. Fluorescent images were taken with and without cells to confirm the absence of imaging artefacts due to the topographical features. To perform automated image analysis in CellProfiler, a robust pipeline was built to objectively analyze every cell from an image set of at least five images, consisting of no less than 100 cells per condition.

After illumination corrections, nuclei morphology was captured using the Otsu adaptive thresholding method applied on the DAPI staining. Subsequently, cell morphology was determined using Otsu adaptive thresholding and appropriate propagation algorithms. Fifteen cell features were extracted including area, compactness, eccentricity, Euler number, extent, form factor, axis length (major and minor), Feret diameter (max and min), radius (max, mean and median), orientation, perimeter, and solidity. Pixel measurements were converted toward the micrometre dimension using a conversion factor of $0.3 \mathrm{~mm} /$ pixel.

\section{Gene expression analysis}

ATDC5 cells were seeded at $5 \times 10^{3}$ cells per $\mathrm{cm}^{2}$ and cultured on seven topographical and a flat (control) surface in BM or ITS medium for 1 or 2 weeks. This experiment was performed in triplicate. RNA was isolated using the RNA II nucleospin RNA isolation kit (Machery Nagel) following the manufacturer's protocol. RNA concentrations were measured using a ND100 spectrophotometer (Nanodrop1000). Reverse transcription was performed with an iScript cDNA synthesis kit according to the manufacturer's protocol. qPCR was performed using $50 \mathrm{ng}$ of cDNA, $0.4 \mathrm{mM}$ of each forward and reverse primer (Sigma Genosys), and 1x SensiMix SYBR and Fluorescein master mix (Bioline). Primer sequences are shown in Supp Table 1.

Quantitative polymerase chain reaction (qPCR) was performed in a My IQ5 machine (Biorad). Data were analyzed using the fit point method of My IQ5 software with the baseline 
set automatically at the lower log-linear part and the cycle threshold (Ct value) was determined. $\mathrm{Ct}$ values were normalized to the Beta-2 microglobulin (B2m) housekeeping gene and $\mathrm{DCt}(\mathrm{Ct}$, control-Ct, sample) was used to calculate the fold change in gene expression.

\section{Statistical analysis}

Data analysis and plotting were performed in R22 and GraphPad Prism version 6.02 for Windows, GraphPad Software, La Jolla, CA, www.graphpad.com. Cell and nuclear morphological features were scaled by subtracting mean and division by SD. To perform clustering analysis, a dissimilarity matrix was calculated from scaled features in the Euclidean space. For performing hierarchical clustering, Ward's algorithm was implemented. Statistical significance of morphological features and gene expression data of different topographies compared to control was analyzed by analysis of variance followed by Tukey HSD test. For pvalue plot, Mann-Whitney $U$ test was applied and $\mathrm{p}$ values were adjusted using Benjamini and Hochberg correction methods.

\section{Model training}

To find unique morphological parameters of cells growing on T2-PS-3322 compared to the other topographies, we used classification trees algorithms from the "rpart" package implemented in R. To explore this further we created a machine learning model. To compensate for differences in cell numbers between conditions, the pooled topography data were downsampled by random selection to have an equal number of data between datasets. To exclude possible bias that might be introduced during downsampling, the model was trained 100 times. Models were trained with 10-fold cross validation in the "caret" package using random forest algorithm. Model accuracy was checked on a subset of the data that was not used for training. The classification tree was visualized using the "party" package.

\section{Results}

\section{Surface micro-topographies guide cell and nucleus shape}

It has been shown before that surface topographies (micro and nano) directly control cell morphology [23-25]. Here, we showed that our surface micro-topographies molded ATDC5 cells into many different sizes and shapes. The cells were cultured in BM on seven different topographical surfaces (Fig. 1a) on discs with a $1.5 \mathrm{~cm}$ diameter with flat PS as a control surface. Topographical surfaces were annotated with their unique feature identifier, derived from the TopoChip database. After 24 hours for the cells to adapt and spread on the surfaces, samples were fixed and stained with Phalloidin and 4'-6-diamidino-2-phenylindole (DAPI) to visualize cell and nucleus structure.

Representative pictures of ATDC5 cells grown on the different surfaces are shown in Fig. 1b. In general, the cell and nucleus shape on micro-topographical surfaces were distinctively affected by the topographies. Fig. 2a shows the most common types of cell shapes found on every surface. On the control flat PS surface, ATDC5 cells displayed their characteristic 
epithelial-like morphology, with unpolarized spreading. On topographical surfaces, the actin filaments were more intensely stained, particularly the parts that were in direct contact with the topography features. Interestingly, on T2-PS-2702, two distinct types of cells could be observed, one was similar to the cells on the control surface while the other resembled the pattern of the topographical feature (Fig. 2a- T2-PS-2702). There were also cells that appeared to be in the transition between control-surface-like to topography-like (Fig. 2a - T2-PS-2702, top right).

Representative nuclear types on each surface are shown in Fig. 2b. Apart from the control surface, where nuclei displayed their regular textbook oval shape, the nuclei on other surfaces appeared to be irregular. In most cases, these nuclei took shape while they had to settle between the topographical features.

For T2-PS-2702 and T2-PS-0205, several distinct types of nuclei existed on one topographical surface. The transitioning from control-surface-like to topography-like of cells on T2-PS-2702 could also be seen with their nuclei (Fig. 2b-T2-PS-2702). Surprisingly, even after 2 weeks of culture this nuclear shape variation remained (Fig. 3), despite that cell shape became unrecognizable because of over-confluency (data not shown). In short, ATDC5 cells and their nuclei exhibited a variety of size and shape when cultured on our micro-topographical surfaces.

\section{Quantification of size and shape features by image analysis}

To extract cell and nuclear shape information, we constructed a CellProfiler pipeline to automatically analyze every cell in a dataset of at least 100 cells for each topographical surface. Fig. 4a represents cell and nuclear morphology quantification in the form of scaled connected dot plots, while Fig. 4b illustrates the statistical significance of morphological features of surfaces compared to flat. In total, 30 parameters were calculated that described both cell and nuclear morphology in great detail.

Almost no significant differences in cell parameters could be observed between the control surface and T2-PS-2702, which is due to the variability caused by two distinct cell types. Regarding cell size, cells on control surface had the biggest size while scoring highest on the cell size measurements perimeter, radius (maximum, mean and median), minor axis length, and minimum Feret diameter. Cells with the smallest surface belonged to T2-PS-3322, T2-PS-0304, and T2-PS-1642.

Concerning nuclei, those found on flat surface were among the biggest together with nuclei from T2-PS-2702, T2-PS-1901, and T2-PS-3240. Nuclei on T2-PS-3322, T2-PS-0304, T2-PS0205 , and T2-PS-1642 continued to be the smallest. Eccentricity is a measure of how elliptic cells are, where 0 represents a circle and 1 a line. Eccentricity of cells on T2-PS-3322 was highest, and so did the nuclei of these cells. The orientation graphs (Fig. 5) illustrates the orientation of cells and nuclei on different surfaces. Cells and nuclei on the flat surface did not align in any particular direction. Only cells from T2-PS- 3322 and T2-PS-0304 were orientated on each surface. Nuclei on T2-PS-3322 also followed the same direction. Overall, these measurements allowed the quantification of unique morphologic features induced by our topographies. 
a
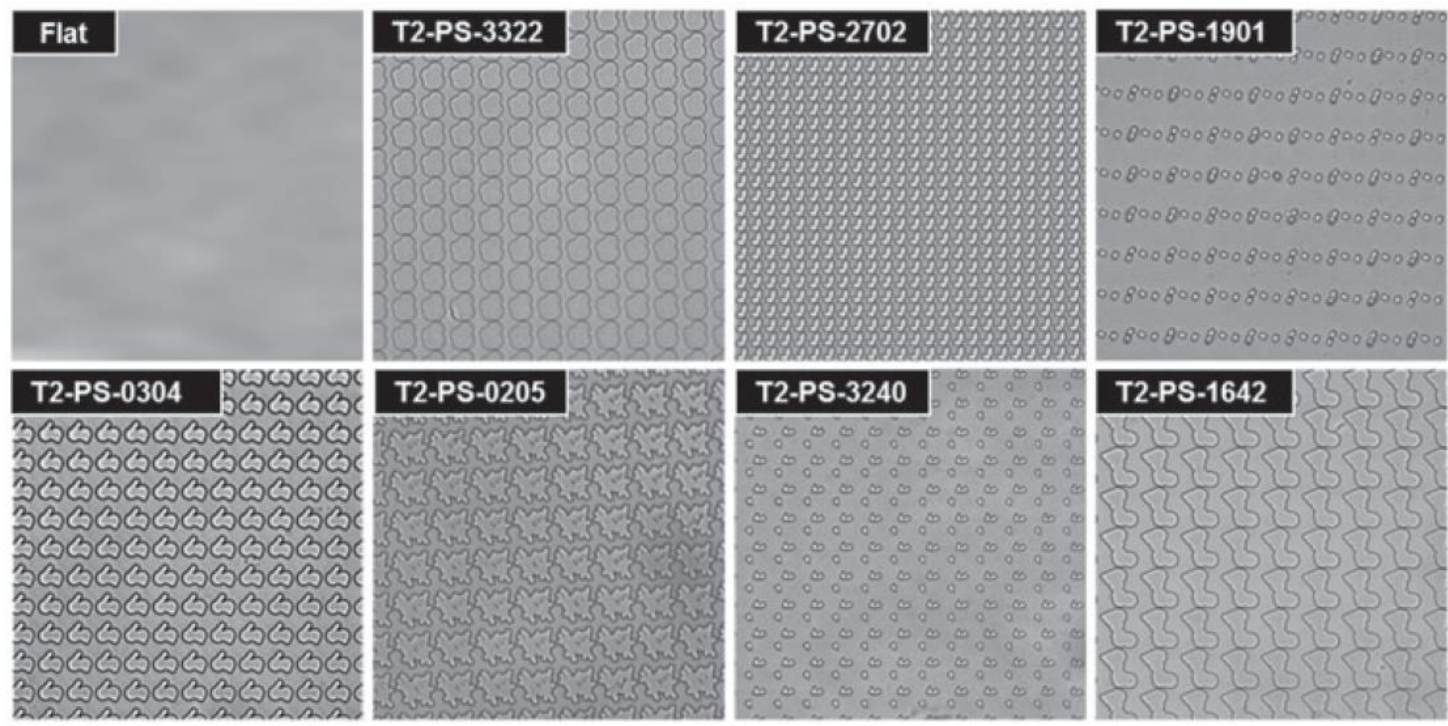

\section{T2-PS-3240}

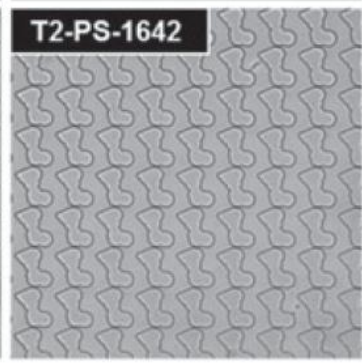

b
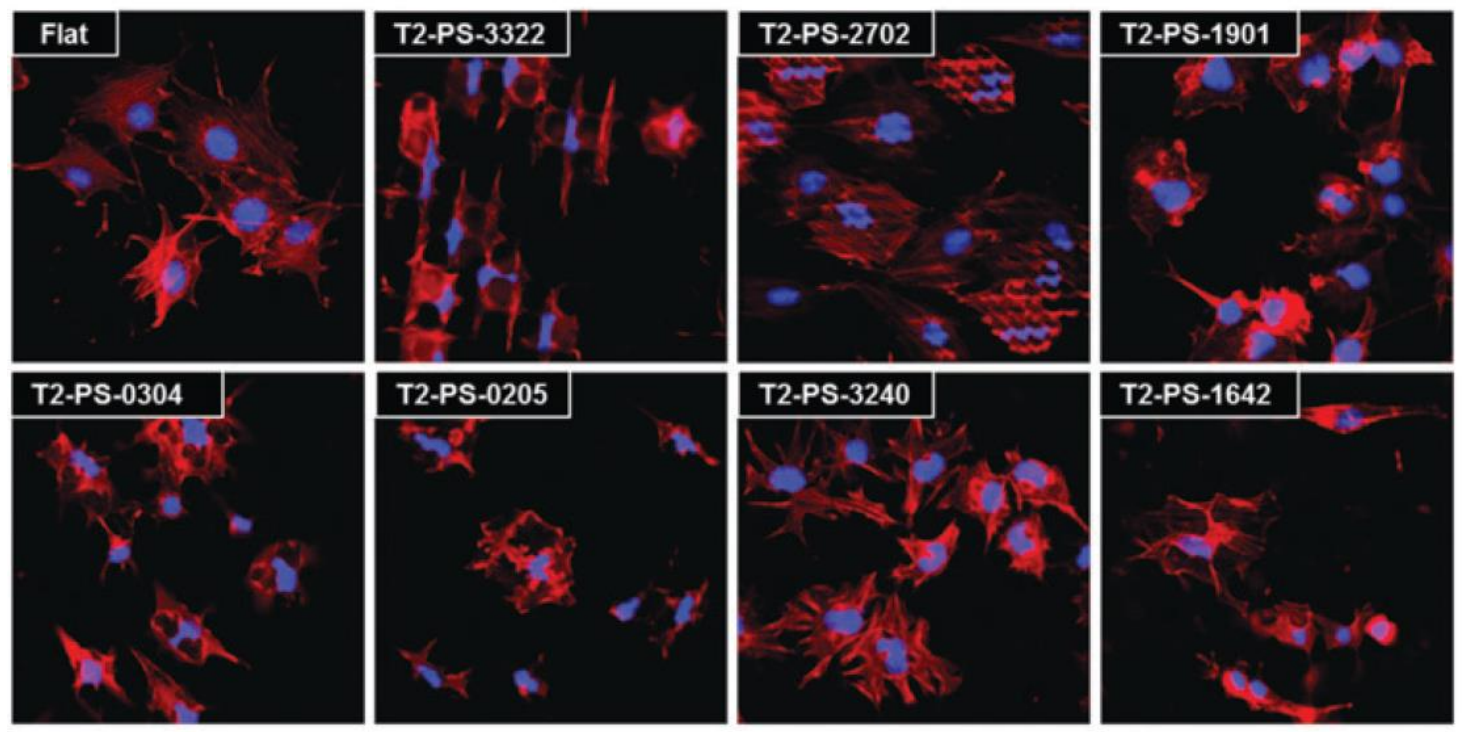

FIG. 1. Visualization of ATDC5 cells grown on distinct surface topographies. (a) Bright-field images of the selected surface topographies. (b) Composite images of ATDC5 cells show the effect of micro-structures on cell and nuclear morphology. Co-staining with markers for nucleus (DAPI) and actin (Phalloidin) were performed $24 \mathrm{~h}$ after seeding. The scale bar represents $50 \mu \mathrm{m}$. 
a
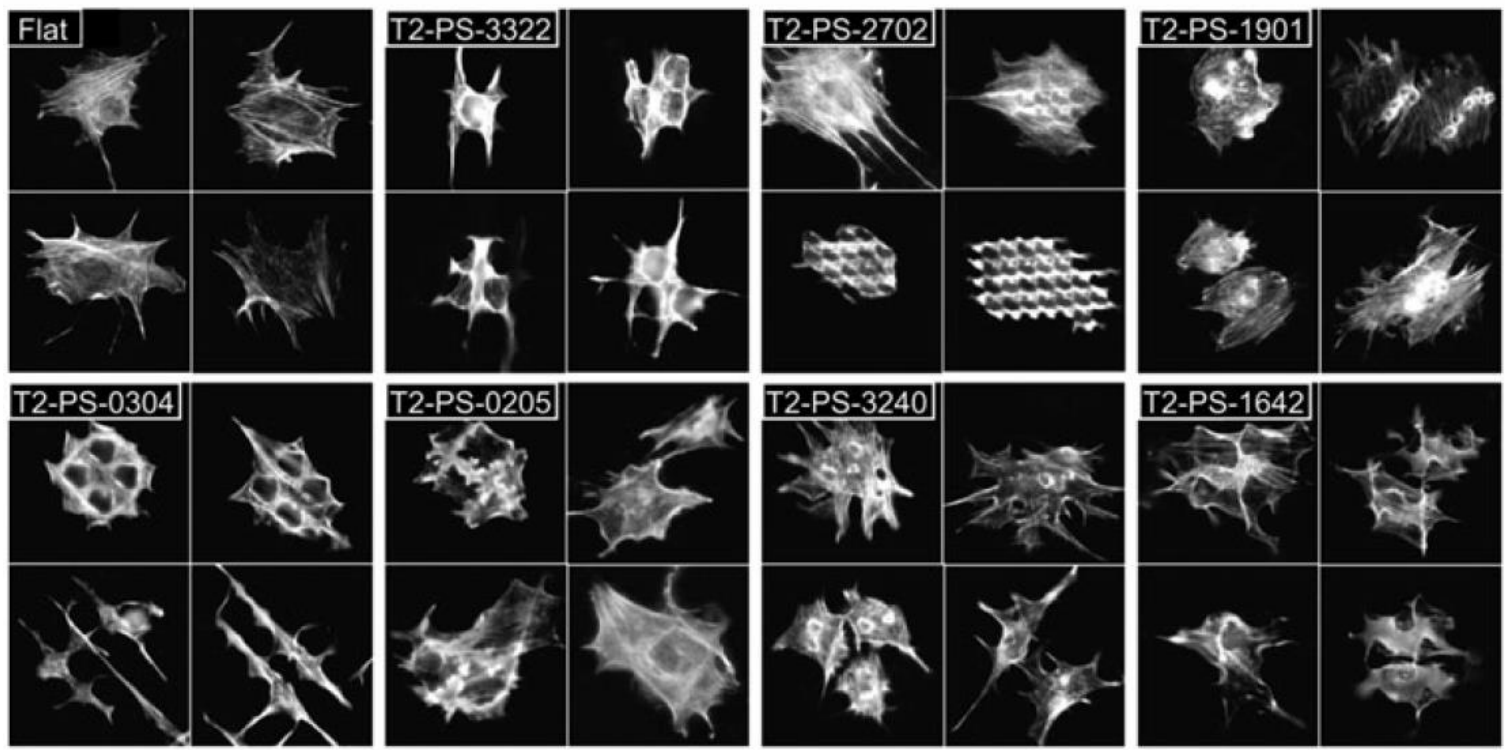

b
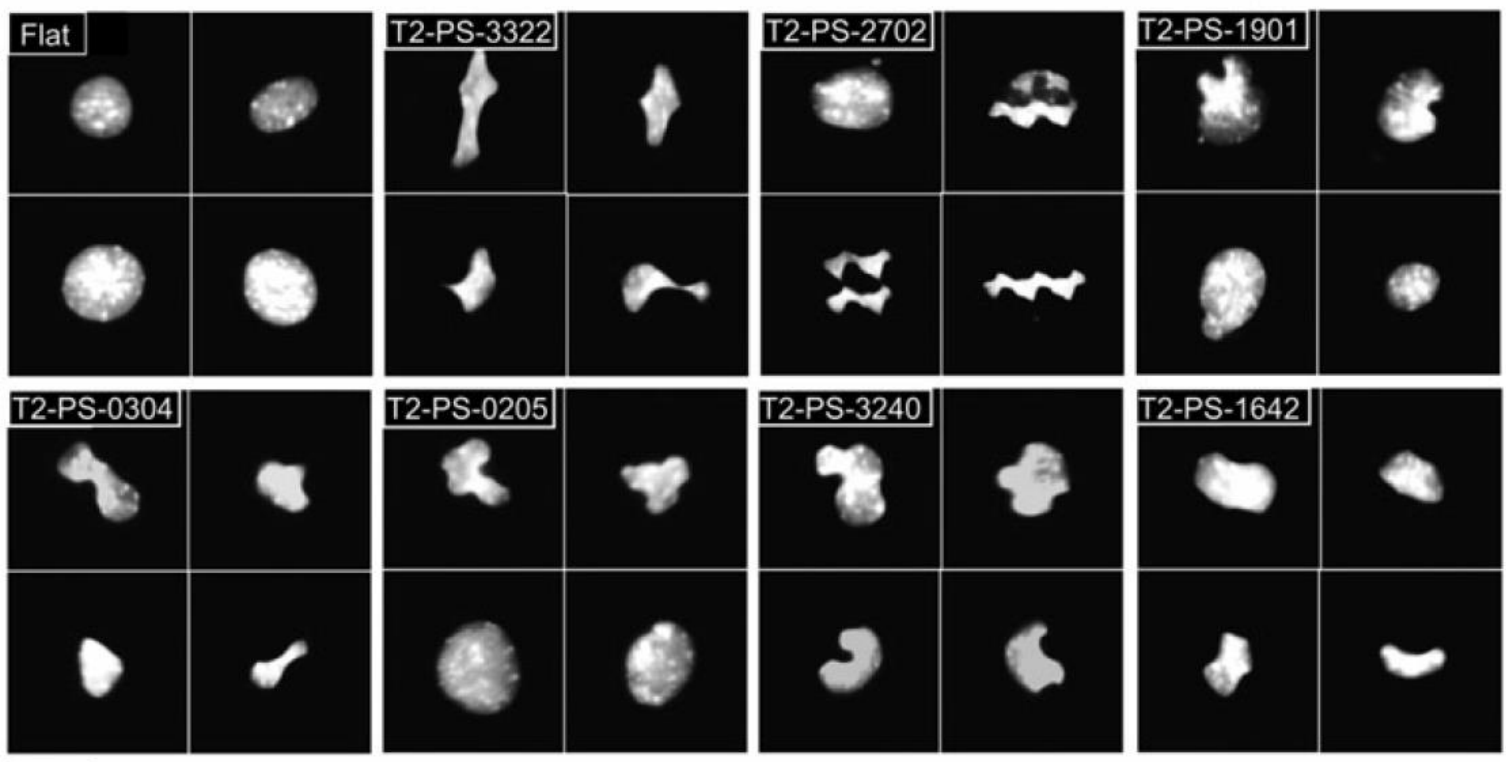

FIG. 2. Visualization of ATDC5 cell and nuclear morphological diversity after culturing $24 \mathrm{~h}$ in BM. Distinct cell and nuclear morphology are present in T2-PS-2702 and T2-PS-0205. (a) Immunocytochemistry with a marker for actin (Phalloidin). The scale bar represents $50 \mu \mathrm{m}$. (b) Marker for nuclei (DAPI). The scale bar represents $25 \mathrm{~mm}$. BM, basic medium; PS, polystyrene. 


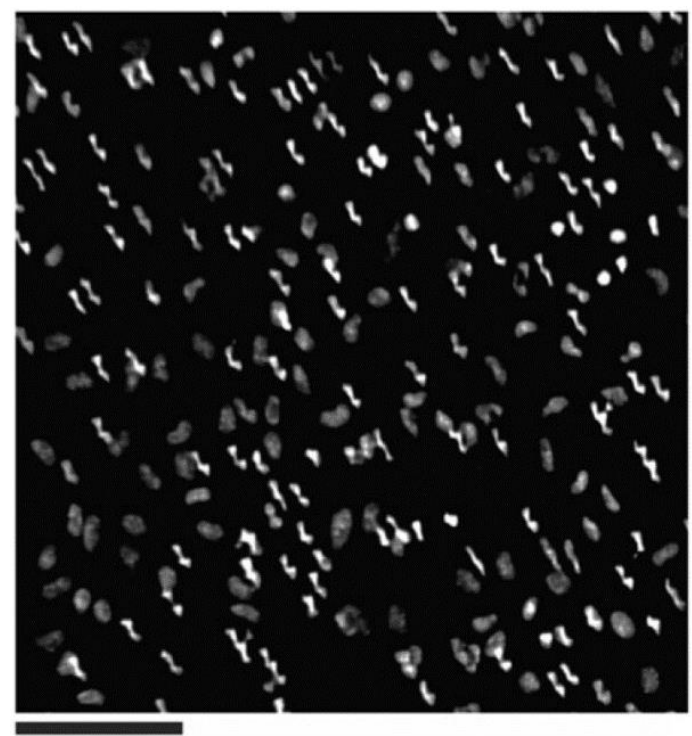

FIG. 3. Long-term culturing of ATDC5 on T2-PS-2702 reveals a consistent pattern of two distinct nuclei. DAPI staining was performed 2 weeks after culturing. Scale bar represents $100 \mu \mathrm{m}$.

ATDC5 cells can spontaneously form cell aggregates (nodules) on common tissue culture surface when stimulated with growth factors such as insulin. To evaluate whether ATDC5 nodule formation would still be possible on micro-topographies, we cultured ATDC5 cells on our topographical surfaces for 2 weeks in the presence of ITS. Fig. 6 shows representative brightfield pictures of cell nodules on all surfaces. In general, nodule formation of ATDC5 cells on these surfaces was uneventful. Without any topography to interfere, the control surface nodules appeared mostly round. Of note, we noticed that on T2-PS-3322, the nodules elongated and aligned in one direction, which was the same direction with the alignment of the topographical units (Fig. 6a-T2-PS-3322 and Fig. 6b). Despite having the same way of topographical unit alignment, T2-PS-2702 and T2-PS-1642 did not guide ATDC5 nodules in any particular direction.

\section{$\underline{\text { Topographies influence expression of chondrogenic genes }}$}

To analyze the effects of topographies on ATDC5 cells in further detail, we performed gene expression analysis for chondrogenesis-related genes on cells grown on different surfaces in either basic or ITS medium for 1 or 2 weeks. Markers for early chondrogenesis (Figs. 7 a-d, collagen II, aggrecan, Sox9, and Hif1a) and late chondrogenesis (Figs. 7 e-h, collagen X, Alp, Mmp13, and Hif2a) were analyzed.

At week 1, Mmp13 expression on all Topo surfaces, apart from T2-PS-2702, was higher than on flat surface in BM. However, this effect diminished when cells were treated with ITS, where only T2-PS-3322 and T2-PS-1642 remained significant. Similarly, Alp expression on T2-PS-0205, T2-PS-3240, and T2-PS-1642 was higher than on control surface in basic but not in ITS medium. For Hif2a, a slight increase was observed for T2-PS-0205 in BM. This effect was more pronounced after ITS treatment, causing a significant Hif2a upregulation for T2-PS3322, T2-PS-1901, T2-PS-0205, T2-PS-3240, and T2-PS-1642. 


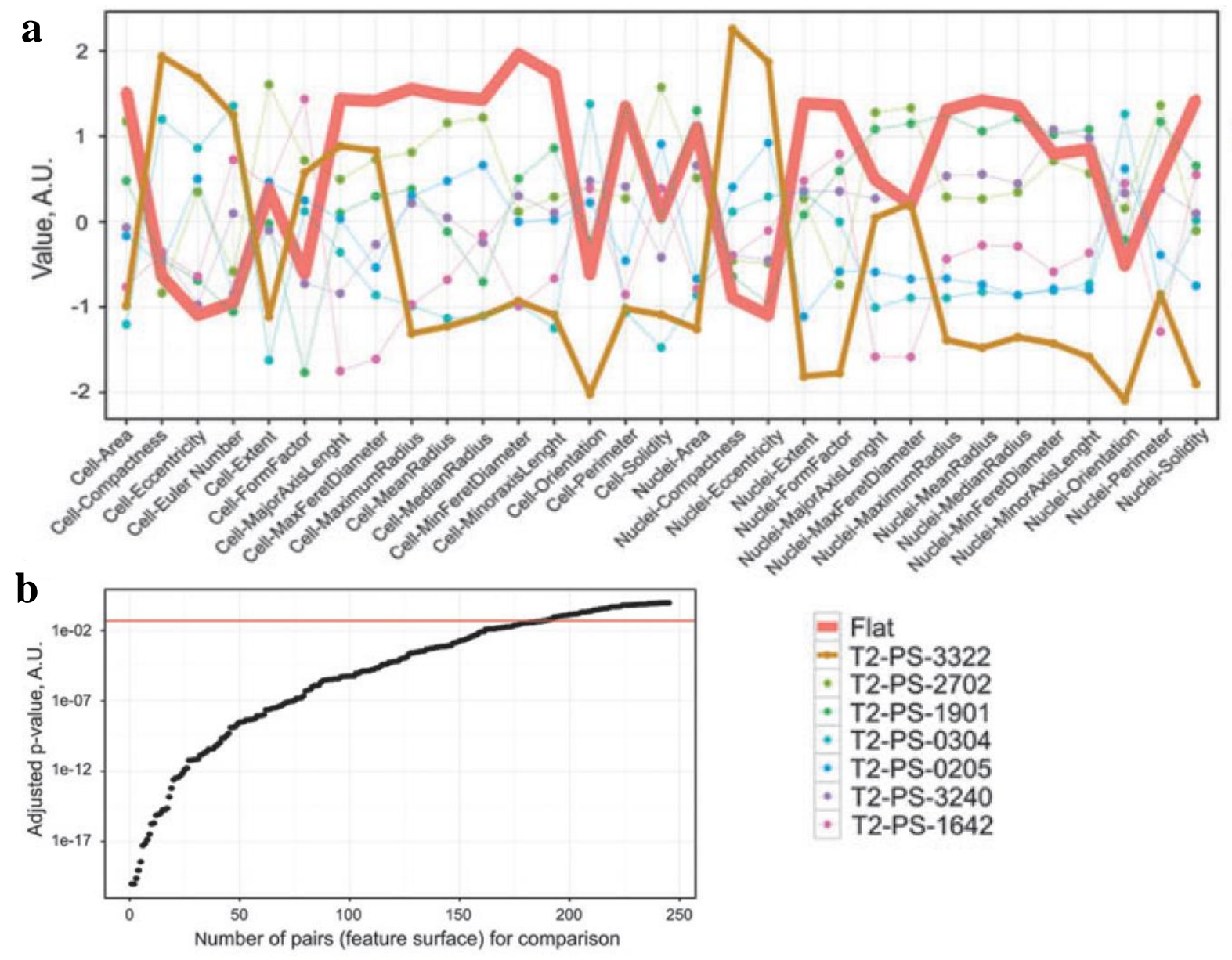

FIG. 4. CellProfiler analysis of cell and nuclear morphology. (a) Connected dot-plot of scaled morphological features highlighting flat and T2-PS-3322. (b) p-Value plot of morphological feature comparison of topographies against flat. p-Values are sorted from lowest to highest. Red line represents the adjusted p-value, everything below is considered significant. 


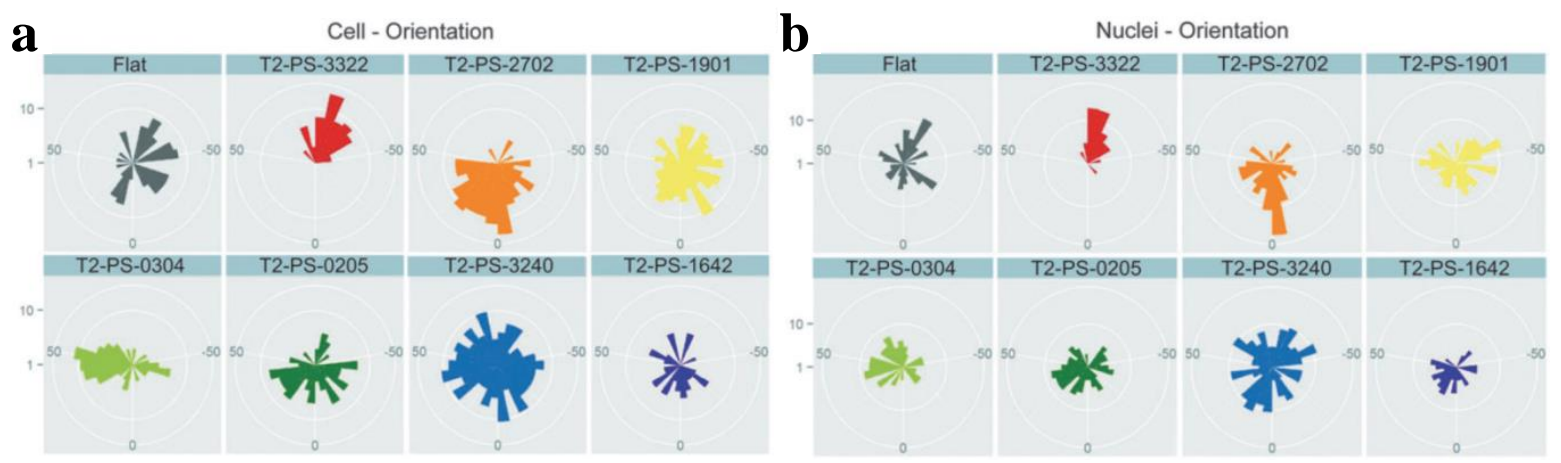

FIG. 5. Orientation of ATDC5 (a) cell and (b) nuclei grown on different surfaces in BM for $24 \mathrm{~h}$. Stained with Phalloidin and DAPI and analyzed by CellProfiler. Data were plotted in R. Cells from T2-PS-3322 and T2-PS-0304 showed orientation on each surface. Nuclei on T2-PS-3322 also followed the same orientation.

a
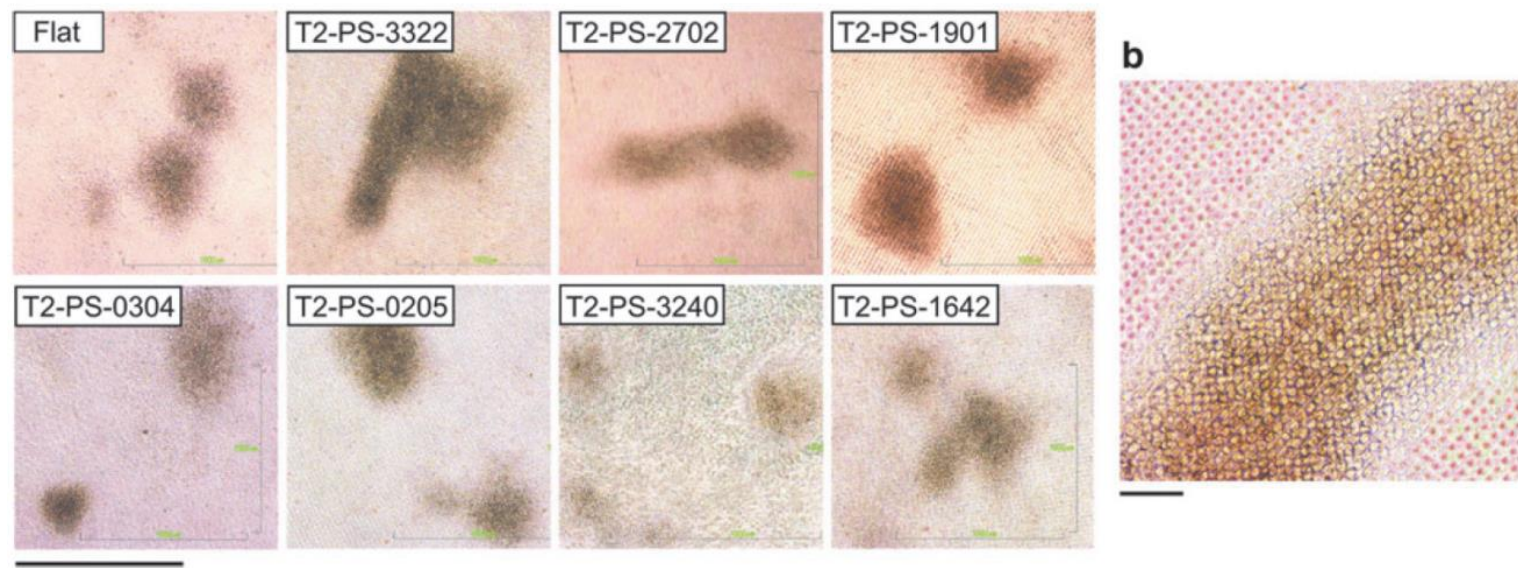

FIG. 6. Bright-field images of ATDC5 nodule formation on topographical surfaces. ATDC5 cells were cultured for 2 weeks in ITS medium. (a) Representative bright-field images of ATDC5 nodules on different surfaces, scale bar represents $1 \mathrm{~mm}$. (b) Nodule elongation on surface T2-PS-3322 aligned to the TopoUnit placement, scale bar represent $100 \mu \mathrm{m}$. 
a

b
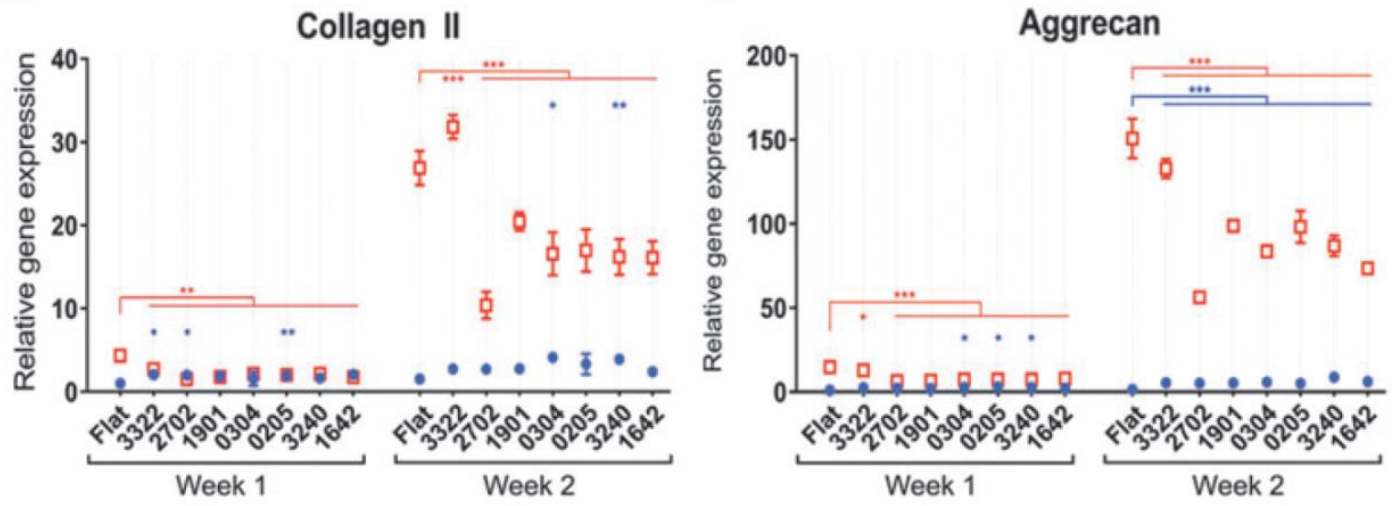

C

d
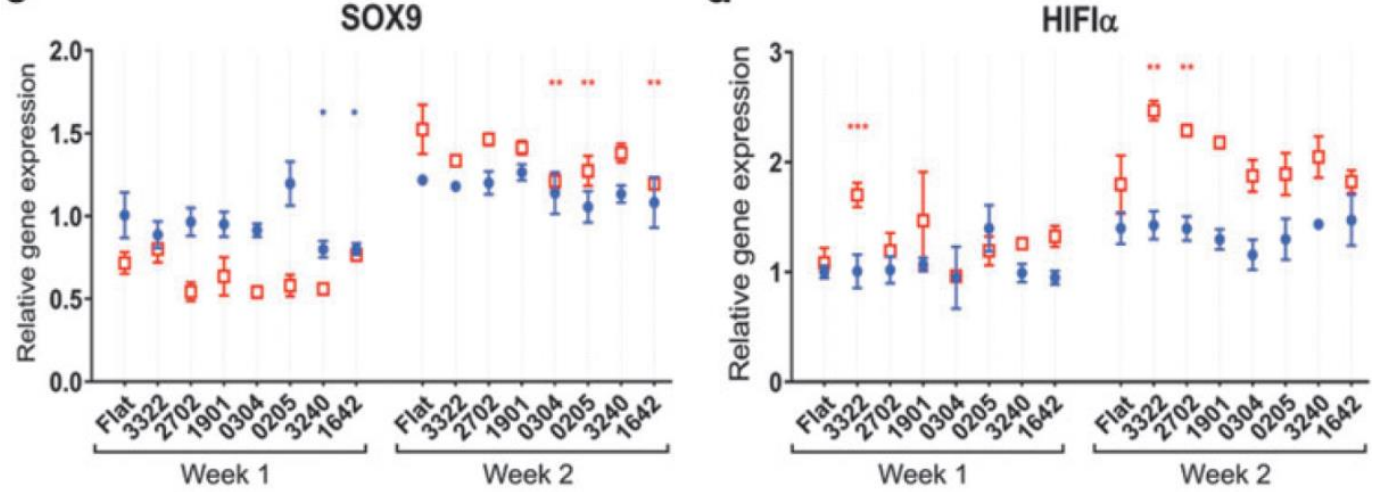

e

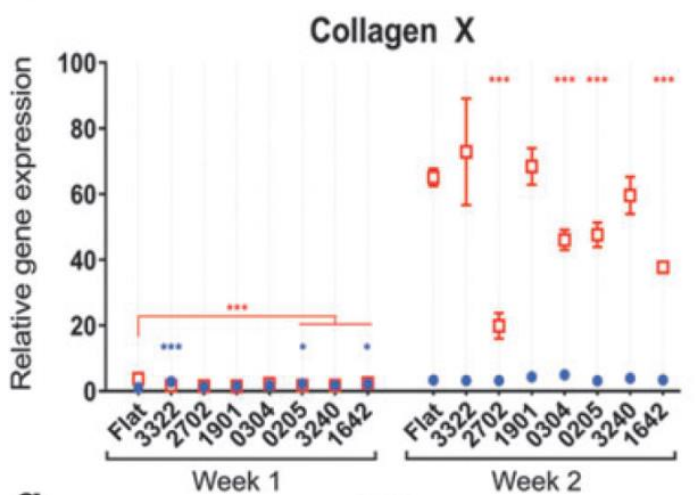

f

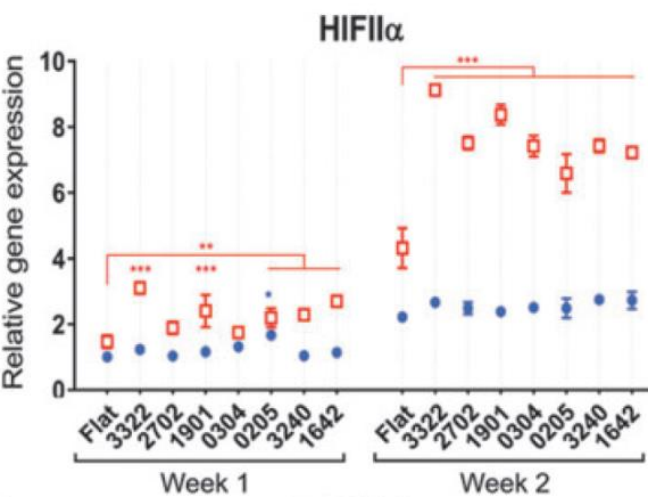

g
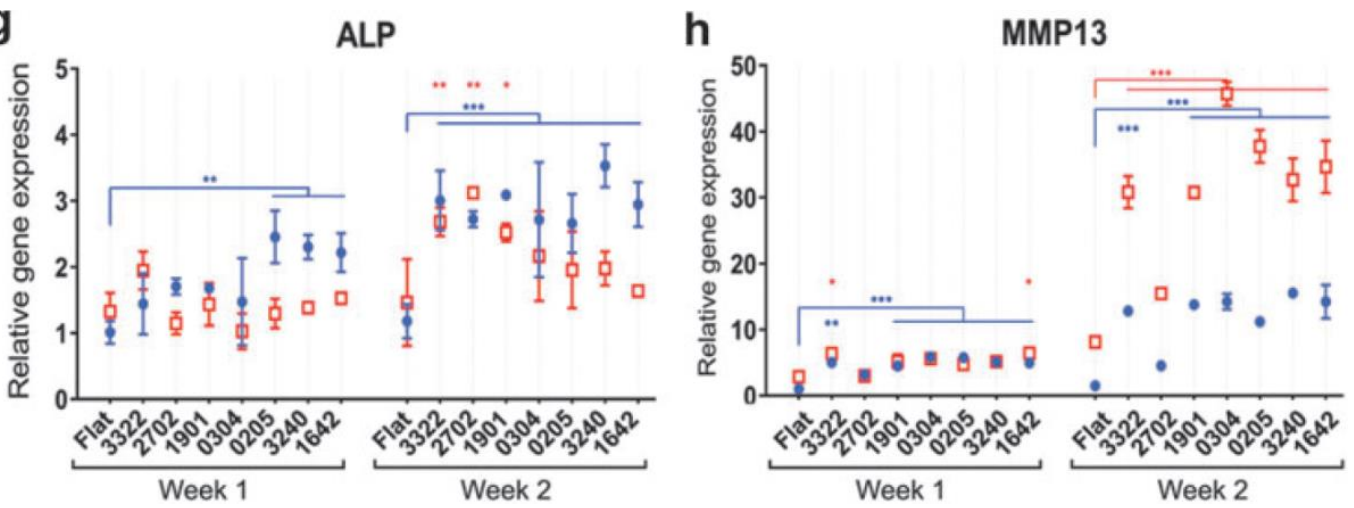

84

- Basic medium a ITS medium 
4 FIG. 7 Gene expression analysis for ATDC5 cells cultured for 1 or 2 weeks on topographical surfaces in basic (blue annotated) and ITS medium (red annotated). qPCR analysis for early chondrogenic markers (a) collagen type II, (b) aggrecan, (c) Sox9, (d) Hif1a and late chondrogenic markers, (e) collagen type X, (f) Hif2a, (g) alkaline phosphatase (Alp), and (h) Mmp13. Besides a few exceptions, little differences are observed at week 1 among surfaces. At week 2, the differences in gene expression among control and Topo surfaces became more pronounced when incubated together with ITS medium. Alp and Mmp13 expression on Topo surfaces outperformed the control surface in both basic and ITS medium. Error bars represent standard deviation $(\mathrm{n}=3) . * \mathrm{p}<0.05, * * \mathrm{p}<0.01, * * * \mathrm{p}<0.001$.

At week 2, the differences in gene expression among the control and Topo surfaces were more pronounced. Expression of chondrogenic genes upregulated over time and was higher in ITS medium. In BM, the effect of topography on chondrogenic gene expression was mild; little difference was seen between topographical surfaces and control surface except for Alp and Mmp13, where expressions on Topo surfaces were all higher, aside from T2-PS-1901. In ITS medium, the difference in gene expression among Topo and control surfaces were greater. Most Topo surfaces induced expression of the late markers Hif2a, Alp, and Mmp13, but inhibited expression of the early markers collagen II, aggrecan, and in some cases Sox9.

In general, gene expression profiles of cells on different Topo surfaces were distinct from one another, which was the same observation as for cell and nuclei shape features. Topo surfaces induced higher expression of late chondrogenic markers (except for collagen X), but inhibited expression of early markers compared to the control surface.

\section{Correlation of morphology and gene expression}

To find out which topography imposed the most significant changes in either cell morphology or gene expression, we performed principal component analysis. For morphology data, we considered the median of all morphological features per surface. Gene expression data were visualized using the mean of the biological triplicate of all genes in both basic and ITS medium for each surface. Surface similarity based on cell and nuclear morphology was visualized by hierarchical dendrogram clustering (Fig. 8a). Cell and nuclear morphologies could be clustered into two distinct groups. In the first group, cell and nuclear morphologies from the control surface were different from T2-PS-2702, T2-PS-1901, and T2-PS-3240. In the other, it was T2-PS- 3322 vs. T2-PS-0304, T2-PS-0205, and T2-PS-1642. In the plot for morphology data (Fig. 8b), T2-PS-3322 was very well separated from all other surfaces in the first two principal components (PC1 and PC2). T2-PS-3322 stood out as the surface that was most distinct in cell and nuclear morphologies compared to other surfaces according to image analysis by CellProfiler.

The same analysis was performed for gene expression data (Figs. 8c-d). The control surface was well separated from all topographies in the first principal component (PC1), while T2-PS3322 was separated in the second principal component (PC2). Hierarchical clustering revealed that the control surface was substantially different from the others in inducing gene expression. Among the Topo group, T2-PS- 3322 was again well separated from the other topographical 
surfaces. Thus, together with the morphological data analysis, T2-PS-3322 was unique in influencing both cell morphology and chondrogenic gene expression of ATDC5. This separation of T2-PS-3322 in gene expression profiles appeared to be caused by the upregulation of both early and late chondrogenic markers.
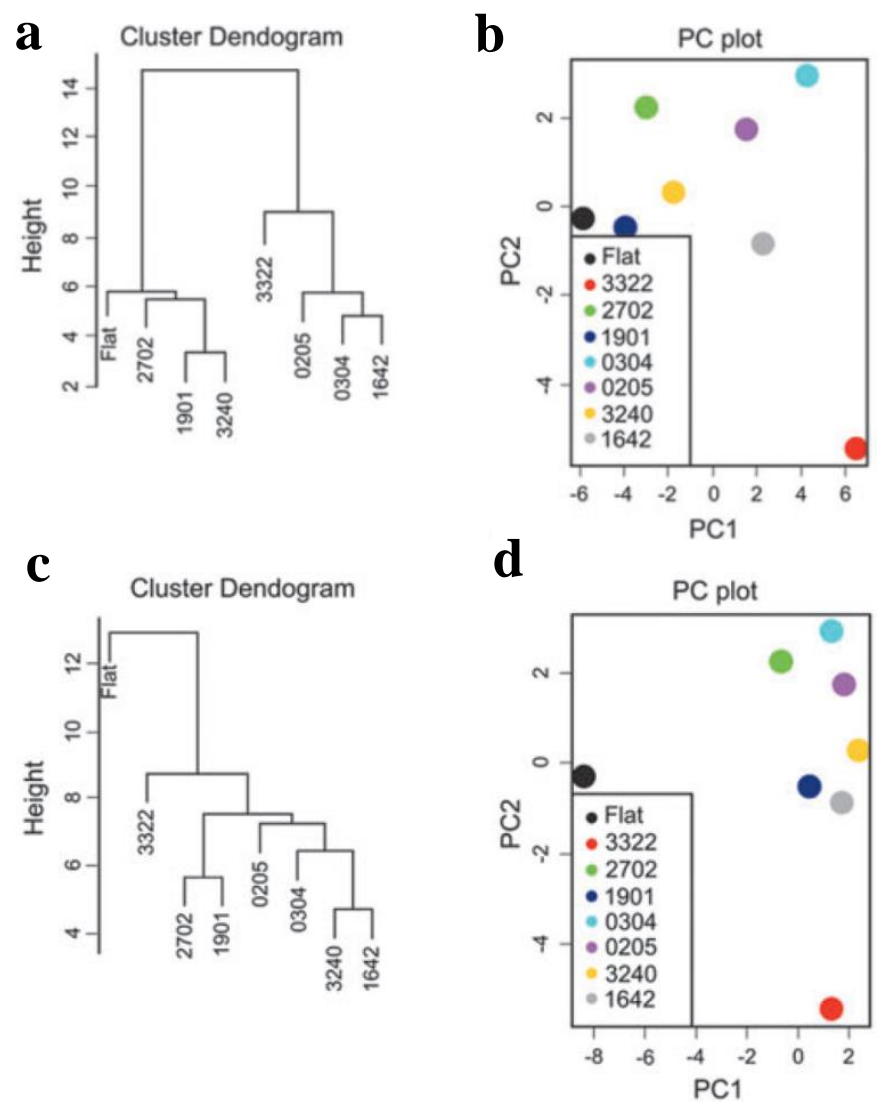

FIG. 8. Cluster dendrogram and principal component (PC) plots identified T2- PS-3322 as most unique in influencing both cell morphology and gene expression. (a) Cluster dendrogram of morphological data showed clustering into two distinct groups. In the first group, the control surface was distinct from T2-PS- 2702, T2-PS-1901, and T2- PS-3240. In the second, T2- PS3322 was separated against T2-PS-0304, T2-PS- 0205, andT2-PS-1642. (b) PC analysis of morphological data revealed separation of T2-PS-3322 from other surfaces. PC plot for morphological data was calculated using median of morphological features per surface. (c) Cluster dendogram for gene expression separated control and T2-PS- 3322 against the other topographical surfaces. (d) PC analysis of gene expression data separated the control surface in the first PC and T2-PS-3322 in the second PC. For gene expression data, PC plots were calculated using the mean of the biological triplicate.

To understand which morphological features makes cells on T2-PS-3322 unique, we constructed a classification tree based on morphology data (Fig. 9a). We used this classification 
tree to understand which cell shape parameters were related to the cells on T2-PS-3322. Almost $90 \%$ of cells with cell compactness $\geq 1.97$ and nuclei maximum Feret diameter $\geq 19.84 \mu \mathrm{m}$ belonged to T2-PS-3322. This means that cells on T2-PS-3322 shared the largest variation in distance between cell border and center than cells on other surfaces. The maximum Feret diameter is a measure of nuclear length; it was suggested that nuclei on T2-PS-3322 were elongated, supported by Fig $\mathbf{2 b}$.

About $70 \%$ of cells with a maximum Feret diameter $<19.84 \mu \mathrm{m}$ and a cell minor-axis-length $<10.75 \mu \mathrm{m}$ were also found on T2-PS- 3322. Cell minor-axis-length also attributed to cell shape narrowness and elongation. Cells that were not as thick as the previous group were less eccentric $(<0.82)$ and at the same time the cell maximum radius was less than $5.85 \mu \mathrm{m}$. Sixty percent of these cells were on T2-PS-3322. These might be mitotic cells. Last major set of parameters of which discriminates cells with $60 \%$ accuracy on T2-PS-3322 are as follows: cell compactness $<1.97$, Euler number $>21.5$, cell minor axis length $>32.8 \mu \mathrm{m}$, and a nuclei maximum radius $>4.0 \mu \mathrm{m}$. The combination of these factors suggested that these cells have an irregular shape with big nuclei; these are apparently cells lying on top of the patterns. Furthermore, we trained 100 machine learning models to distinguish cells morphology from T2PS-3322 and the other topographies. We found that the most important morphological feature for that analysis was the nuclei mean radius (Figs. 9b-c). The mean accuracy of these models was $81 \%$ (Fig. 9d), which indicates that cell response on T2-PS-3322 was unique and can be accurately predicted using cell morphological features.

\section{Discussion}

In the growth plate, proliferation and differentiation of chondrocytes are tightly controlled by soluble factors such as Indian hedgehog, parathyroid hormone-related protein, and bone morphogenetic proteins [26-28]. Likewise, ECM protein receptors on chondrocytes, such as $\beta 1$-integrins, regulate cell arrangement into the regular longitudinal column of proliferative chondrocytes [29]. Mechanical loading also plays an important role in controlling the growth of this hypertrophic plate $[30,31]$. It is known that cell shape is important for chondrocyte function [32,33], however, little is known about the influence of surface topography, or rather ECM topography, on the development of chondrocytes in the body. When cells are exposed to a foreign environment, it is inevitable that they lack their usual trophic signals. Here, material surface topography becomes an inherent foreign signal existing in all artificial systems. Once harnessed, surface topography can then be utilized as an instructive signal for chondrocyte growth and hypertrophic differentiation.

In this study, we investigated the effect of seven designed surface micro-topographies on the chondrogenic differentiation process of ATDC5 cells. These seven topographies were selected from the hits of our previous high-throughput screen for topographies. In this previous screen, four of these hits induced ALP secretion of hMSCs (T2-PS-3322, T2-PS-0304, T2-PS-0205, and T2-PS-1642), while three did not show any influence (T2-PS-2702, T2-PS-1901, and T2PS-3240). Note that in this study, ALP expression is induced differently by our topographies. 


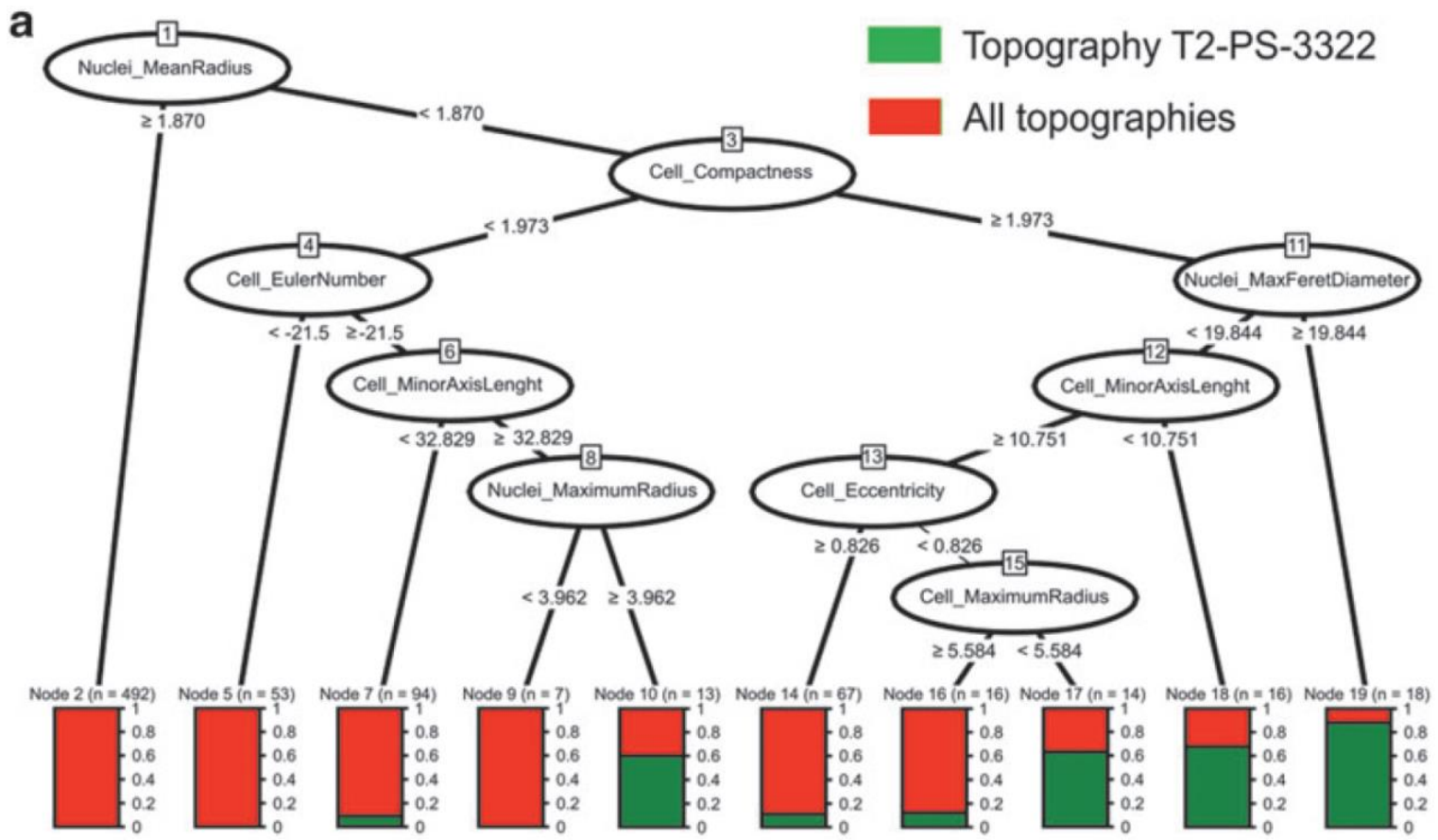

b Nuclei_MeanRadius. Nuclei_MaximumRadius. Nuclei_MinorAxisLength. Nuclei_Eccentricity. Nuclei_Compactness. Nuclei_MinFeretDiameter. Nuclei_Solidity. Cell Compactness. Cell EulerNumber. Nuclei_FormFactor. Nuclei_Extent. Nuclei_Area.

Cell MaximumRadius. Cell Extent. Cell_Solidity. Cell MeanRadius. Cell_Eccentricity. Nuclei Perimeter. Cell FormFactor. Cell_Perimeter. Cell Area. Cell MaxFeretDiameter. Cell_MinorAxisLength. Nuclei_MajorAxisLength.

Cell_MajorAxisLength. Nuclei $\bar{M}$ axFeretDiameter. Cell MinFeretDiameter. Cell_MedianRadius.

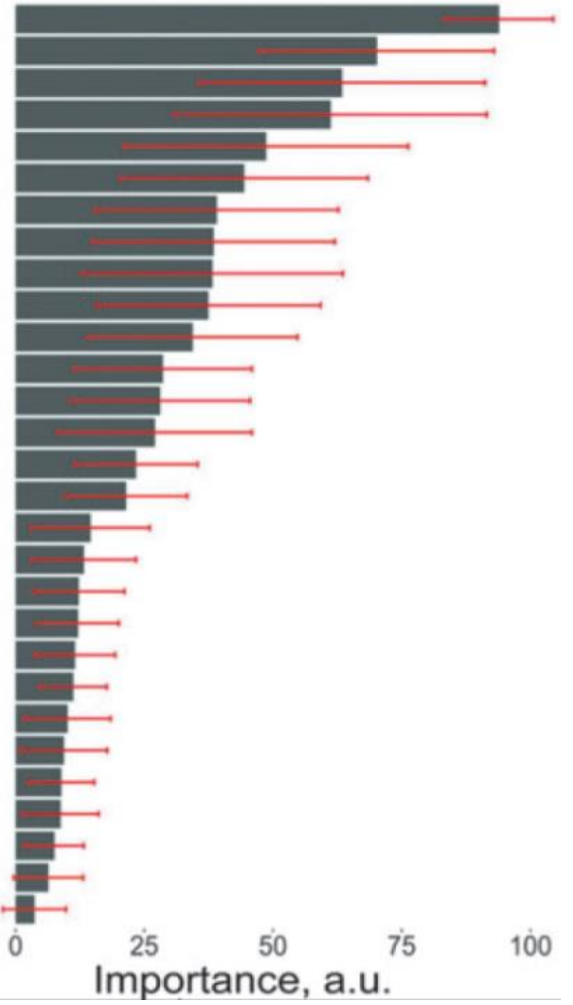

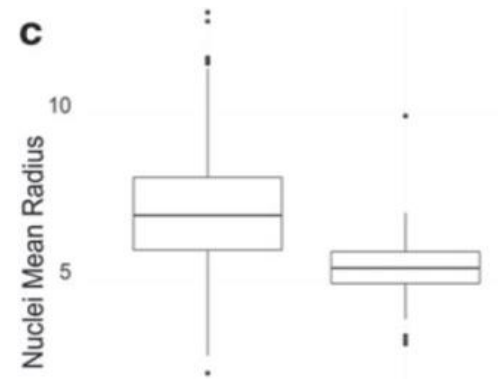

All Topographies Topography 3322

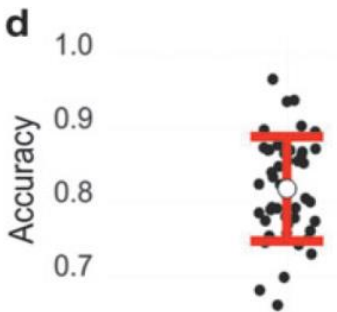

0.6 
4 FIG. 9. Identification of unique morphology features of cells grown on T2-PS-3322. (a) Classification Tree showing the sorting of topographies by cell and nuclear morphology parameters. The nuclei mean radius is identified as the most important property that makes these cells distinct from cells grown on others surfaces. (b) Importance of cell and nuclear morphology parameters for predicting T2-PS-3322 in 100 Random Forest classification models. Bar chart represents mean standard deviation. (c) Distribution of the nuclei mean radius for T2-PS-3322 and other pooled topographies. (d) Accuracy prediction of 100 Random Forest models.

This likely reflects the different cell type used or the influence of different surface chemistry on cell behavior. ALP is often used as an in vitro marker for osteogenic differentiation of MSCs, but ALP is also a marker for hypertrophic differentiation of chondrocytes [34, 35]. In this study, we show that ATDC5 cells cultured on the Topo surfaces can significantly influence the expression of ALP and other late markers of chondrogenesis, supporting our hypothesis that surface topography can influence the differentiation of chondrocytes.

Chondrocyte aggregation in vitro is a process mimicking mesenchymal condensation in vivo. Keeping the cell-to-cell contact has been shown to be a prerequisite of chondrogenesis. Hamilton et al. used a micro-topography silica substrate with grooves measuring 0.75 to $8 \mu \mathrm{m}$ in depth and 5 to $12.5 \mu \mathrm{m}$ in width - to control aggregation of primary chondrocytes [36]. They noted that larger aggregates collided with each other on the groove and often in parallel to the direction of the groove. We also found that ATDC5 grown on T2-PS-3322 formed aggregates, which elongated in parallel to the alignment of the topographical patterns. Although all seven Topo surfaces had the same pattern arrangement, the ATDC5 aggregate elongation only appeared on T2-PS-3322, suggesting that the size of the pattern itself affected this observation. The implications of nodule alignment can be the subject of further studies.

In vitro, cell morphology is paramount to cell growth, as demonstrated by Folkman and Moscona in one of the earliest experiments proving the relationship between cell shape and DNA synthesis [4]. In their experiments, the cell shape changed from flat to spheroidal as the result of reduced adhesion to the culture plate. Since our topographical surfaces were made of PS, the standard tissue culture material, cells adhered very well and thus the topography often served as a mold encasing the cells. Often, actin was very intensely stained around the wall of the topography or in the grooves created by the topography patterns. The cell nuclei also adapted the shape of the topography. CellProfiler analysis showed a large variation in cell and nuclei features among the seven Topo surfaces. Interestingly, our gene expression set followed the same trend. To elucidate the effect of cell morphology on gene expression, our strategy involved analyzing a dataset of 30 cell morphological features and correlating these with a gene expression dataset of early and late markers for chondrogenesis. The problem remains that gene expression analysis is based on averaging gene expression of all the cells on one single surface, which at times can vary considerably. The use of single-cell polymerase chain reaction may be the answer to overcome this problem.

We conclude that the use of micro-topographies can influence the expression of early and late markers for chondrogenesis. In combination with soluble factors, these topographies can be 
used to control chondrogenic differentiation of ATDC5 cells in vitro. Since our research was based on only seven topographies, future studies with different topographies and chemistry could further fine-tune the optimal surface architecture for hypertrophic differentiation.

\section{Acknowledgments}

J.d.B. acknowledges the financial support of the Dutch province of Limburg, F.H. the NanoNext initiative, A.V. the European Union's Seventh Framework Programme (FP7/ 20072013; Grant agreement 289720), and S.V. the European Union's Horizon 2020 Programme (H2020-MSCAITN- 2015; Grant agreement 676338). We appreciate the financial support for our studies as part of the PIDON project (PID091014) issued by the Dutch State, the Ministry of Economic affairs.

\section{Disclosure Statement}

C.A.v.B. and J.d.B. are co-founders of, and have a financial interest in, Materiomics b.v. The other authors do not have any financial interests to declare.

\section{References for Chapter 5 (Topography)}

1. McBeath, R., Pirone, D.M., Nelson, C.M., Bhadriraju, K., and Chen, C.S. Cell shape, cytoskeletal tension, and RhoA regulate stem cell lineage commitment. Dev Cell 6, 483, 2004.

2. Dalby, M.J., Gadegaard, N., Tare, R., Andar, A., Riehle, M.O., Herzyk, P. Wilkinson, C.D.W., and Oreffo, R.O.C. The control of human mesenchymal cell differentiation using nanoscale symmetry and disorder. Nat Mater 6, 997, 2007.

3. Marklein, R.A., and Burdick J.A. Controlling stem cell fate with material design. Adv Mater 22, 175, 2010.

4. Folkman, J., and Moscona, A. Role of cell shape in growth control. Nature 273, 345, 1978.

5. Damanik, F.F.R, Rothuizen, T.C., van Blitterswijk, C.A., Rotmans, J.I., and Moroni, L. Towards an in vitro model mimicking the foreign body response: tailoring the surface properties of biomaterials to modulate extracellular matrix. Sci Rep 4, 6325, 2014.

6. Yang, L., Hedhammar, M., Blom, T., Leifer, K., Johansson, J., Habibovic, P., and van Blitterswijk, C.A. Biomimetic calcium phosphate coatings on recombinant spider silk fibres. Biomed Mater 5, 045002, 2010.

7. Shibata, Y., and Tanimoto, Y. A review of improved fixation methods for dental implants. Part I: surface optimization for rapid osseointegration. J Prosthodont Res 59, 20, 2015.

8. Wennerberg, A., and Albrektsson T. Effects of titanium surface topography on bone integration: a systematic review. Clin Oral Implants Res 20 Suppl 4, 172, 2009.

9. Albrektsson, T., Branemark, P.I., Hansson, H.A., and Lindstro"m, J. Osseointegrated titanium implants. Requirements for ensuring a long-lasting, direct bone-to-implant anchorage in man. Acta Orthop Scand 52, 155, 1981. 
10. Unadkat, H.V., Hulsman, M., Cornelissen, K., Papenburg, B.J., Truckenmu“ller, R.K., Carpenter, A.E., Wessling, M., Post, G., Uetz, M., Reinders, M.J.T., Stamatialis, D., van Blitterswijk, C.A., and de Boer, J. An algorithm-based to- pographical biomaterials library to instruct cell fate. Proc Natl Acad Sci U S A 108, 16565, 2011.

11. Pfander, D., Swobodam, B., and Kirsch, T. Expression of early and late differentiation markers (proliferating cell nuclear antigen, syndecan-3, annexin VI, and alkaline phosphatase) by human osteoarthritic chondrocytes. Am J Pathol 159, 1777, 2001.

12. Jukes, J.M., Both, S.K., Leusink, A., Sterk, L.M.T., van Blitterswijk C.A., and de Boer J. Endochondral bone tissue engineering using embryonic stem cells. PNAS 105, 6840, 2008.

13. Yao, Y., and Wang, Y. ATDC5: an excellent in vitro model cell line for skeletal development. J Cell Biochem 114, 1223, 2013.

14. Le, B.Q., Fernandes, H., Bouten, C.V.C., Karperien, M., van Blitterswijk, C.A., and de Boer, J.High-throughput screening assay for the identification of compounds enhancing collagenous extracellular matrix production by ATDC5 cells. Tissue Eng Part C Methods 21, 726, 2015.

15. Hatakeyam, Y., Matsuda Y., Hatakeyama J., Oka K., Anan H., Tsuruga E., Inai T., Ishikawa H., and Sawa Y. The effect of growth differentiation factor-5, 6, 7 in chondrogenic cell differentiation of ATDC-5. Am J Biosc 2, 182, 2014.

16. Altaf, F.M., Hering, T.M., Kazmi, N.H., Yoo, J.U., and Johnstone, B. Ascorbate-enhanced chondrogenesis of ATDC5 cells. Eur Cells Mater 12, 64, 2006.

17. Portron, S., Hivernaud, V., Merceron, C., Lesoeur, J., Masson, M., Gauthier, O., Vinatier C., Beck V., and Guicheux J. Inverse regulation of early and late chondrogenic differentiation by oxygen tension provides cues for stem cell-based cartilage tissue engineering. Cell Physiol Biochem 35, 841, 2015.

18. Kwon, H.J., Yasuda, K., Ohmiya, Y., Honma, K., Chen, Y.M., and Gonge, J.P. In vitro differentiation of chondrogenic ATDC5 cells is enhanced by culturing on synthetic hydrogels with various charge densities. Acta Biomater 6, 494-501, 2010.

19. Hulsman, M., Hulshof, F., Unadkat, H., Papenburg, B.J., Stamatialis, D.F., Truckenmu“ller, R., van Blitterswijk C., de Boer J., and Reinders M.J.T. Analysis of high-throughput screening reveals the effect of surface topographies on cellular morphology. Acta Biomater $15,29,2015$.

20. Abagnale, G., Steger, M, Nguyen, V.H., Hersch, N., Sechi, A., Joussen, S., Denecke, B., Merkel, R., Hoffmann, B., Dreser, A., Schnakenberg, U., Gillner, A., and Wagner, W. Surface topography enhances differentiation of mesenchymal stem cells towards osteogenic and adipogenic lineages. Biomaterials 61, 316, 2015.

21. Carpenter, A.E., Jones, T.R., Lamprecht, M.R., Clark, C., Kang, I.H., Friman, O., Guertin, A.D., Chang, J.H., Lindquist, R.A., Moffat, J., Golland, P., and Sabatini, D.M. CellProfiler: image analysis software for identifying and quantifying cell phenotypes. Genome Biol 7, R100, 2006.

22. R Core Team. R: A language and environment for statistical computing. Vienna, Austria: R Foundation for Statistical Computing, 2009.

23. Shah, J.V. Cells in tight spaces: the role of cell shape in cell function. J Cell Biol 191, 233, 2010.

24. Cadart, C., Zlotek-Zlotkiewicz, E., Le Berre, M., Piel, M., and Matthews, H.K. Exploring the function of cell shape and size during mitosis. Dev Cell 29, 159, 2014.

25. Lecuit, T., and Lenne, P.F. Cell surface mechanics and the control of cell shape, tissue patterns and morphogenesis. Nat Rev Mol Cell Biol 8, 633, 2007.

26. Minina, E., Wenzel, H.M., Kreschel, C., Karp, S., Gaffield, W., McMahon, A.P., and Vortkamp, A. BMP and Ihh/PTHrP signaling interact to coordinate chondrocyte proliferation and differentiation. Development 128, 4523, 2001. 
27. Caron, M.M.J., Emans, P.J., Cremers, A., Surtel, D.A.M., Coolsen, M.M.E., van Rhijn, L.W., and Welting T.J.M. Hypertrophic differentiation during chondrogenic differentiation of progenitor cells is stimulated by BMP-2 but suppressed by BMP-7. Osteoarthr Cartil 21, 604, 2013.

28. Chen, X., Macica, C.M., Nasiri, A., and Broadus, A.E. Regulation of articular chondrocyte proliferation and differentiation by indian hedgehog and parathyroid hormonerelated protein in mice. Arthritis Rheum 58, 3788, 2008.

29. Aszodi, A., Hunziker, E.B., Brakebusch, C., and Fa“ssler, R. Betal integrins regulate chondrocyte rotation, G1 progression, and cytokinesis. Genes Dev 17, 2465, 2003.

30. Frost, H.M. Skeletal structural adaptations to mechanical usage (SATMU): 3. The hyaline cartilage modeling problem. Anat Rec 226, 423, 1990.

31. Villemure, I., and Stokes I.A. Growth plate mechanics and mechanobiology. A survey of present understanding. J Biomech 42, 1793, 2009.

32. Glowacki, J., Trepman E., and Folkman J. Cell shape and phenotypic expression in chondrocytes. Proc Soc Exp Biol Med 172, 93, 1983.

33. Guo, H., and Torzilli, P.A. Shape of chondrocytes within articular cartilage affects the solid but not the fluid microenvironment under unconfined compression. Acta Biomater 29, 170, 2016.

34. Goldring, M.B., Tsuchimochi K., and Ijiri K. The control of chondrogenesis. J Cell Biochem 97, 33, 2006.

35. Dreier, R. Hypertrophic differentiation of chondrocytes in osteoarthritis: the developmental aspect of degenerative joint disorders. Arthritis Res Ther 12, 216, 2010.

36. Hamilton, D.W., Riehle, M.O., Monaghan, W., and Curtis, A.S. Chondrocyte aggregation on micrometric surface topography: a time-lapse study. Tissue Eng 12, 189, 2006. 
Supp Table 1. Primer sequences

\begin{tabular}{ll}
\hline Name & Primer sequence \\
\hline B2m: beta-2 microglobulin [mouse] & 5'-CATGGCTCGCTCGGTGACC-3' \\
& 5'-AATGTGAGGCGGGTGGAACTG-3' \\
Col2a1: collagen type II alpha 1 [mouse] & 5'-CAAGGCCCCCGAGGTGACAAA-3' \\
& 5'-GGGGCCAGGGATTCCATTAGAGC-3' \\
Col10a1: collagen type X alpha 1 [mouse] & 5'-CATAAAGGGCCCACTTGCTA-3' \\
& 5'-TGGCTGATATTCCTGGTGGT-3' \\
Acan: aggrecan [mouse] & 5'-AGAACCTTCGCTCCAATGACTC-3' \\
& 5'-AGGGTGTAGCGTGTGGAAATAG-3' \\
Sox9: SRY-box9 [mouse] & 5'-CCACGGAACAGACTCACATCTCTC-3' \\
& 5'-CTGCTCAGTTCACCGATGTCCACG-3' \\
Hif1a: hypoxia inducible factor 1 alpha [mouse] & 5'-TGCTCATCAGTTGCCACTTC-3' \\
& 5'-TGGGCCATTTCTGTGTGTAA-3' \\
Hif2a: hypoxia inducible factor 2 alpha [mouse] & 5'-TGAGTTGGCTCATGAGTTGC-3' \\
& 5'-CTCACGGATCTCCTCATGGT-3' \\
Alp: alkaline phosphatase [mouse] & 5'-AACCCAGACACAAGCATTCC-3' \\
& 5'-GAGACATTTTCCCGTTCACC-3' \\
Mmp13: matrix metallopeptidase 13 [mouse] & 5'-AGGCCTTCAGAAAAGCCTTC-3' \\
& 5'-TCCTTGGAGTGATCCAGACC-3' \\
\hline
\end{tabular}




\title{
Chapter 6 \\ Generation of a BMP-2 reporter MG63 cell line using CRISPR/Cas9 mediated homologous recombination
}

\author{
Bach Q. Le, Clemens van Blitterswijk, Jan de Boer, Vanessa LS LaPointe
}

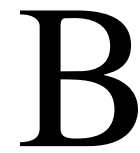

one morphogenetic protein 2 (BMP-2) is a growth factor, also known as a cytokine and as a metabologen, originally identified as an important cartilage and bone inducer. BMP-2 is now known to be involved in a wide range of functions, from orchestrating tissue architecture to maintaining metabolism and homeostasis. To study BMP-2 function, many biological tools have been developed including the BMP-2 conditional knockout mouse (Bmp2 ${ }^{\text {floxneo }}$ ) and the immortalized floxed BMP-2 osteoblast cell line. To date, no BMP2 reporter cell line has been established. In this study, we aimed to develop a knock-in reporter MG63 cell line for BMP-2 utilizing CRISPR/Cas9 assisted homologous recombination. A BMP-2 targeting vector was constructed on a linear pJAZZ backbone consisting of a reporter sequence Flag-2A-eGFP-loxP-PGK-puro-loxP flanked by two BMP-2 homologous arms. Four CRISPR vectors, each consisting of a single-guide RNA (sgRNA) and a Cas9 nuclease, were designed to specifically target the 100-400 bp region upstream of BMP-2 stop codon. Once a specific double-stranded break is created, the BMP-2 targeting vector will serve as a template for homology directed repair (HDR), during which the reporter gene sequence will be copied into the original chromosome. Although the BMP-2 targeting vector was successfully constructed and validated by sequencing, the CRISPR vectors failed to create a double-strand break in the MG-63 cell line model. More optimization is needed to make the CRISPR/Cas9 effective before homologous recombination with the BMP-2 targeting vector can be archived. 


\section{Introduction}

Bone morphogenetic proteins (BMPs) were discovered by Marshall Urist in 1965 and named for their ability to induce bone and cartilage formation [1, 2]. Originally, seven proteins (BMP-1 to BMP-7) were identified and isolated from bovine bone [3]; since then, 13 more BMPs have been discovered, bringing the total to 20 [4]. BMP signaling is involved in organogenesis and the establishment of the basic embryonic body plan [5]. Of all the BMPs, BMP-2 is the most studied since it was the first manufactured by recombinant technology [6]. BMP-2 has been extensively evaluated in the clinic for bone induction, and recombinant human BMP-2 is used as a complete bone graft substitute in many surgical procedures worldwide.

In addition to bone induction, BMP-2 also has a wide range of other functions. Lyons et al used in situ hybridization to detect BMP-2 mRNA expression in a variety of embryonic epithelial and mesenchymal tissues outside of the skeletal system [7]. High expression of BMP2 was found in the myocardium of atrio-ventricular canal, whisker follicles, tooth bud, otic vesicle, palate, and mesonephric tubules. This suggests that BMP-2 signaling plays a role in tissue development. In 2009, A. Hari Reddi and Anand Reddi proposed the term metabologen to define a morphogen that can initiate, promote and maintain metabolism and homeostasis [8]. According to this, BMP-2 (and other BMPs) is a metabologen involved in iron homeostasis, brown fat adipogenesis and energy metabolism $[9,10]$.

Many efforts have been made to produce biological tools to study the complexity of the BMP-2 network. Zhang and Bradley (1996) generated a deletion of the BMP-2 mature region in the mouse genome and showed that the BMP-2 null mutant died prior to stages of organogenesis due to multiple developmental defects in the extraembryonic mesoderm and the heart [11]. Ma and Martin (2005) generated a BMP-2 conditional null allele (Bmp2 ${ }^{\text {floxneo }}$ ) by introducing LoxP sites upstream and downstream of BMP-2 exon 3 that encodes the mature peptide [12]. This conditional null allele encodes wildtype BMP-2 activity and reverts to a null allele upon Cre recombinase-induced recombination. The availability of the conditional BMP-2 knockout mouse has helped reveal important roles for BMP-2 in the later stage of osteogenesis, fracture healing and other organ development [13-15]. Wu et al (2011) developed an immortalized floxed BMP-2 osteoblast cell line using osteoblasts harvested from calvarial bone of the floxed BMP-2 mice [16]. Such a cell line is a valuable tool to study the effects of BMP-2 on osteoblast cell lineage as well as relevant molecular events involved in extracellular matrix mineralization and bone regeneration.

Apart from deletion, another good strategy to study a gene of interest is to couple it with a reporter gene such as GFP, which will allow the gene activity to be easily identified and measured. Such reporter system is not only a powerful tool for scientists to study the gene, but also a valuable resource for the pharmaceutical or biotech industry where high throughput screening is employed to discover drugs or factors that affect activity of the reported gene. Previously we demonstrated that BMP-2 expression in hMSCs varied significantly when the cells were cultured on different calcium phosphate materials, due to different material chemistry and surface topography $[17,18]$. In this case, a BMP-2 reporter cell line is a very useful tool to set up a high throughput biomaterial screen - a biomedical engineering strategy to search for osteoinductive materials. 
To date, no BMP-2 reporter system has been established. In this study, we attempted to develop a knock-in reporter MG63 cell line for BMP-2 utilizing CRISPR/Cas9 assisted homologous recombination. Using the same strategy with CRISPR/Cas9, many reporter cell lines have been established [19-21]. MG63 is a human osteosarcoma-derived cell line commonly used for osteoblastic models [22, 23]. To report BMP-2 transcriptional activity, we aimed to fuse the gene with a Flag-2A-eGFP-loxP-PGK-puro-loxP sequence. Flag is a DNA sequence developed by Sigma-Aldrich, encoding a short, hydrophilic 8-amino acid peptide. The Flag peptide is likely to be located at the surface of the fusion protein because of its hydrophilic nature, making it an easy target for antibody binding (Anti-Flag, Sigma-Aldrich). The 2A sequence encoded a self-cleaving small peptide first identified by Ryan et al. in the foot-andmouth disease virus (FMDV) [24]. The presence of a 2A sequence results in ribosome skipping and thus cleavage between the $2 \mathrm{~A}$ peptide and its immediate downstream peptide, in this case eGFP. This allows the reporter eGFP to be transcribed and translated at the same time with BMP-2, but cleaved-off during translation. Finally, the selection cassette PGKpuro was sandwiched between two loxP sites, which can be used for excision of the cassette by Cre recombinase upon successful insertion of the reporter sequence.

CRISPR/Cas9 is utilized to make a specific double-strand break between 100-400 bp upstream of the stop codon of BMP-2. This double-strand chromosome break will induce the endogenous repair machinery to re-join the DNA by either non-homologous end joining (NHEJ) or homology directed repair (HDR). The reporter sequence that we want to insert is flanked by two homologous arms that can be used by the cell as templates to repair the broken DNA. As HDR occurs, the reporter gene sequence is copied into the original chromosome.

In this chapter, we describe our attempt to generate the reporter MG63 cell line for BMP-2. Although the cell line was not successfully established, the BMP-2 targeting vector was generated and verified by sequencing, paving the way to development of a BMP-2 reporter system in the future.

\section{Materials and methods}

\section{$\underline{\text { Cell culture }}$}

MG63 cells (ATCC CRL-1427) were maintained in basic medium consisting of MEM alpha with nucleosides, $10 \% \mathrm{v} / \mathrm{v}$ fetal bovine serum (FBS, Lonza), $2 \mathrm{mM}$ L-glutamine, $0.2 \mathrm{mM} \mathrm{L-}$ ascorbic acid-2 phosphate, $100 \mathrm{U} / \mathrm{mL}$ of penicillin and $100 \mu \mathrm{g} / \mathrm{mL}$ of streptomycin. Cells were grown at $37^{\circ} \mathrm{C}$ in a humid atmosphere with $5 \% \mathrm{CO} 2$. Medium was refreshed 2-3 times per week and cells were used for further subculturing or cryopreservation upon reaching near confluence. Selection medium consisted of basic medium supplemented with $0.5 \mu \mathrm{g} / \mathrm{ml}$ puromycin and 10 $\mu \mathrm{g} / \mathrm{ml}$ ganciclovir. A titration assay was performed to determine puromycin concentration for MG63, which was the lowest concentration that resulted in 100\% cell death after 3-5 days. The effective concentration of ganciclovir for negative selection of MG63 clones was taken from literature [25]. All supplements were purchased from ThermoFisher Scientific and all chemicals were purchased from Sigma-Aldrich unless stated otherwise. 


\section{$\underline{\text { Targeting vector construction }}$}

The targeting vector backbone was a linear cloning plasmid (pJAZZ, Lucigen \#43036). This linear plasmid system is not subject to supercoiling in the bacterial cells, thus facilitating the cloning of unstable sequences that are AT-rich or contain inverted repeats [26]. In this case, the homologous right arm contained a large number of AT-rich repeat sequences which proved difficult to clone using a circular plasmid.

The cloning sequence comprised of tk-left_arm-insert-right_arm, where the insert was Flag2A-eGFP-loxP-PGK-puro-loxP. To construct this sequence, the homologous arms were PCRcloned from the genomic DNA of MG63 using a high-fidelity Taq polymerase (NEB \#M0494). The left arm (LA) was the $1.5 \mathrm{~kb}$ sequence at the 5 '-end of the stop codon (not including the stop codon) of BMP-2 gene at locus NG_023233 on chromosome 20. The right arm (RA) was the $1 \mathrm{~kb}$ sequence at the 3 '-end of the stop codon and included the stop codon. The Flag sequence (GATTACAAGGACGACGATGACAAG) and a NheI restriction site was incorporated at the 3'-end of the LA using a reverse primer (see Supplementary table 1 for primer sequences). Similarly, an AscI restriction site was incorporated at the 5'-end of the RA using a forward primer. The PCR products were checked for correct size and purified using a PCR purification kit (Qiagen).

The 2A-eGFP-loxP-PGK-Puro-loxP fragment $(2.6 \mathrm{~kb})$ was obtained from pOCT4-2AeGFP-PGK-Puro (Addgene plasmid \#31938 [27]). The fragment was cut from the OCT4 vector at NheI and AscI sites and separated by electrophoresis on a $0.5 \%$ agarose gel with Sybr Safe (1:10,000; ThermoFisher). The $2.6 \mathrm{~kb}$ band was cut out and purified using a gel extraction kit (Qiagen). After that, the fragment was ligated to LA-Flag and RA in the correct order at the NheI and AscI sites. The sequence (LA-insert-RA) was ligated into pJAZZ at SmaI site. Finally, the negative selection (tk) sequence was cut from pAL119-TK (Addgene plasmid \#21911 [28]) at the XbaI site. The cut DNA was separated and cleaned up as described above, then blunted and ligated into the pJAZZ at ApaI site. The final vector was verified by sequencing (BaseClear, the Netherlands).

\section{CRISPR target sites and CRISPR vector design}

The CRISPR targeting region was 100-400 bp before the stop codon. Cas9 target sites were identified and four sgRNAs were designed to target this region (CRISPR-Cas9 service by GeneCopoeia). The four target sites were (A) CGGGGTATCACGCCTTTTAC, (B) GTACGTGGACTTCAGTGACGT, (C) AACACAAACAGCGGAAACGC, and (D) TCATGCCATTGTTCAGACGT. Each sgRNA was combined with its specific CBh-driven Cas9 nuclease in a single vector (pCRISPR-CG02, GeneCopoeia). The Cas9 nuclease used in this project was a wild type enzyme that would produce double-strand breaks. All CRISPR vectors were sequenced to ensure the presence of the right sequence.

\section{$\underline{\text { SURVEYOR assay }}$}

CRISPR vectors were tested on MG63 cells using transfection. Briefly, $2 \mu \mathrm{g}$ of each CRISPR vector was transfected to $1 \times 10^{6}$ MG63 cells by electroporation (Amaxa Cell Line Nucleofector Kit C, Lonza). After three days, DNA from the targeted and non-targeted cells was isolated and PCR-amplified (Phusion high-fidelity polymerase, NEB \#M0530) for the 
targeted region (see primer set Surveyor in table 1). Then, equal amounts of PCR products from targeted and non-targeted cells were hybridized using a thermocycler to allow heteroduplex formation. After that, the annealed heteroduplex DNA was treated with SURVEYOR nuclease (Transgenomic) and DNA fragments were separated by electrophoresis on a $1 \%$ agarose gel to identify the presence of the expected bands.

\section{Generation of BMP-2 reporter MG63 clones, selection and validation}

MG63 cells were expanded for a few days and after harvesting, $1 \times 10^{6}$ cells were electroporated (Amaxa Cell Line Nucleofector Kit C, Lonza) using $8 \mu \mathrm{g}$ of DNA consisting of each CRISPR vector plus the targeting vector at 1:1, 1:2 or 1:3 ratio. After electroporation, cells were expanded for 3 days and selection was done by adding positive and negative selection $(0.5$ $\mu \mathrm{g} / \mathrm{ml}$ puromycin and $10 \mu \mathrm{g} / \mathrm{ml}$ ganciclovir) for 2 weeks. After the selection, surviving clones were picked for expansion and PCR screening. PCR primers were designed to span outside the homologous left arm up to GFP region to ensure proper site-specific targeting (see validation primers at Supp Table 1). Upon confirmation of a correct insertion, the whole region will be PCR-amplified and sequenced to ensure in-frame positioning of the reporter gene.

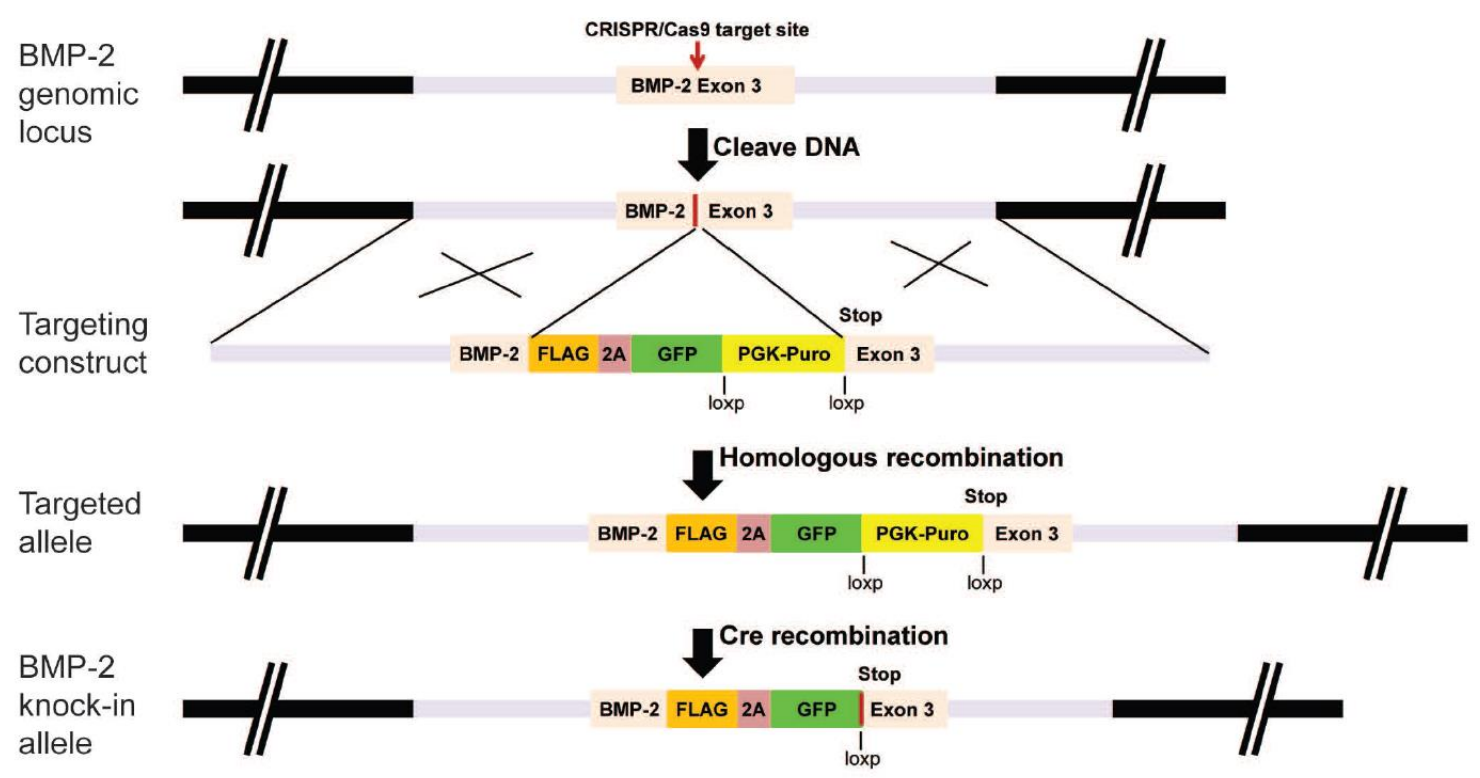

FIG. 1. Schematic showing the steps to generate a BMP-2 reporter cell line utilizing CRISPRCas9 and HDR at BMP-2 locus. CRISPR-Cas9 complexes make DSB 100-400 bp before the stop codon of BMP-2. HDR incorporates the insert, consisting of FLAG-2A-GFP-PGK-Puro, from the DNA targeting construct (DNA template). Finally, Cre recombination is performed to excite the Puro cassette. 

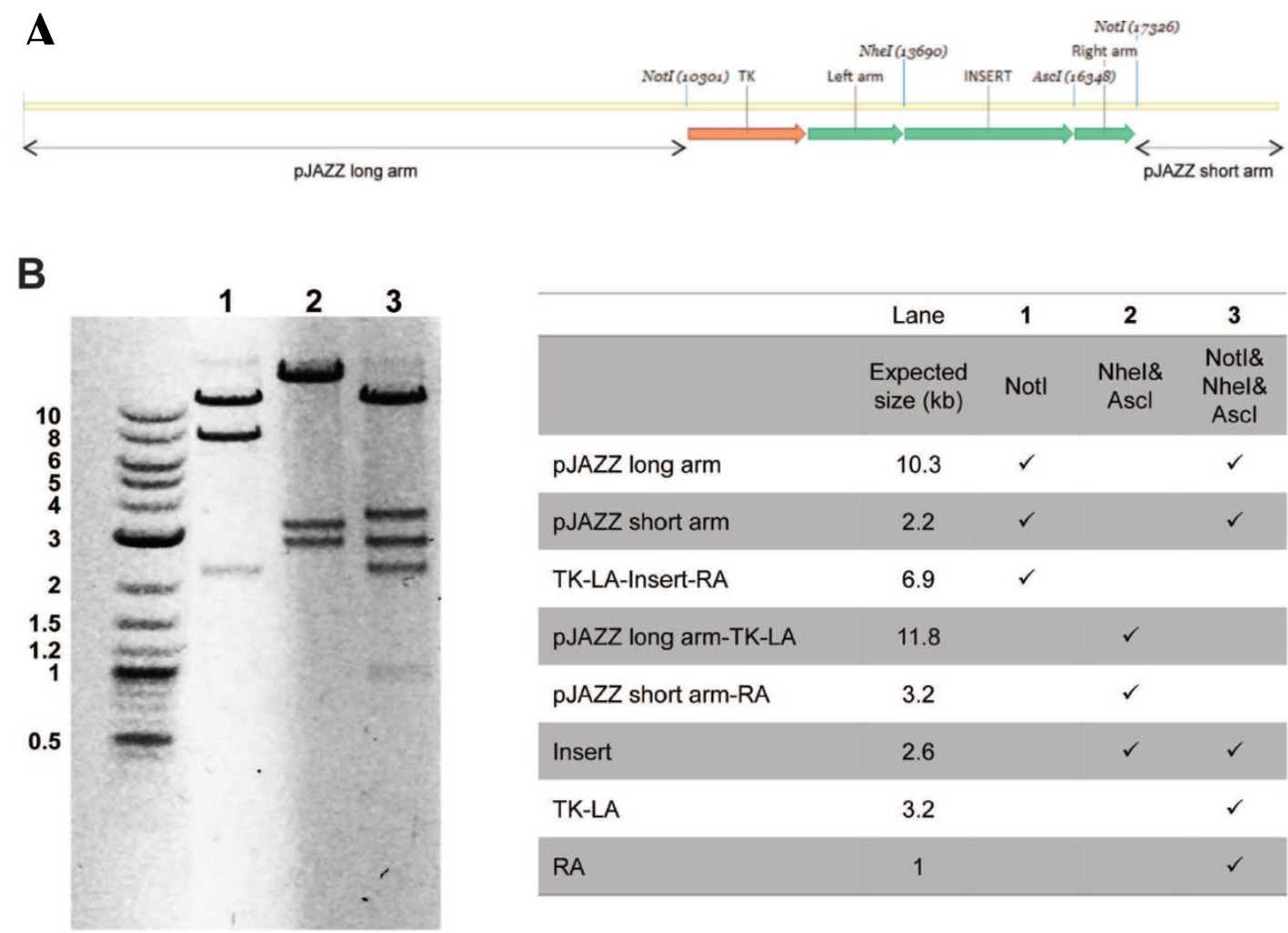

\begin{tabular}{lcccc}
\hline & Lane & $\mathbf{1}$ & $\mathbf{2}$ & $\mathbf{3}$ \\
\hline & $\begin{array}{c}\text { Expected } \\
\text { size (kb) }\end{array}$ & Notl & $\begin{array}{c}\text { Nhel\& } \\
\text { Ascl }\end{array}$ & $\begin{array}{c}\text { Notl\& } \\
\text { Nhel\& } \\
\text { Ascl }\end{array}$ \\
\hline pJAZZ long arm & 10.3 & $\checkmark$ & & $\checkmark$ \\
\hline pJAZZ short arm & 2.2 & $\checkmark$ & & $\checkmark$ \\
\hline TK-LA-Insert-RA & 6.9 & $\checkmark$ & & \\
\hline pJAZZ long arm-TK-LA & 11.8 & & $\checkmark$ & \\
\hline pJAZZ short arm-RA & 3.2 & & $\checkmark$ & \\
\hline Insert & 2.6 & & $\checkmark$ & $\checkmark$ \\
\hline TK-LA & 3.2 & & & $\checkmark$ \\
\hline RA & 1 & & & $\checkmark$ \\
\hline
\end{tabular}

FIG. 2. The targeting construct built on a linear plasmid pJAZZ backbone, consisting of 1.5-kb homologous left arm, the insert sequence FLAG-2A-GFP-PGK-Puro and a 1-kb homologous right arm (A). Validation of the targeting by restriction digestion showing the correct sizes of the expected components $(\mathbf{B})$.

\section{Results and Discussion}

Fig. 1 describes our targeting strategy for generating a BMP-2-FLAG-2A-GFP reporter using CRISPR/Cas9 mediated homologous recombination. The human BMP-2 gene locates at chromosome 20, consisting of three exons encoding a single transcript. The four CRISPR/Cas 9 sgRNAs were designed to target a region 100-400 bp before the stop codon at exon 3 . The Cas 9 nuclease would make a double strand break, stimulating the cell repair machinery to heal its broken DNA. The targeting construct (Fig. 2A), which included a $1.5 \mathrm{~kb}$ homologous left arm and a $1 \mathrm{~kb}$ homologous right arm, would serve as a template for homology directed repair. As this happens, the reporter sequence FLAG-2A-GFP-PGK-Puro would be inserted before the stop codon in exon 3 of the BMP-2 gene. Two loxP sites flanking the PGK-Puro cassette are used for removal of the cassette by CRE recombination upon successful insertion of the reporter sequence.

As we performed this study, there were a number of obstacles that we have yet to overcome. First, the homology right arm was extremely difficult to be PCR-amplified, due to its high AT content. We solved this problem by performing touch-down PCR and a high-fidelity 
polymerase. Then, the right arm again could not be cloned in a regular circular vector due to the sequence being truncated by the bacterial hosts. This was solved by using the pJAZZ linear vector system. The targeting construct was successfully made and verified by restriction digestion (Fig. 2B) and sequencing.

The CRISPR/Cas9 activity was supposed to be detected by the SURVEYOR assay, which failed to show in our experiments. Four CRISPR vectors, each consisting of a specific sgRNA and a Cas9 nuclease, were transfected alone or in combination to MG63. After three days, DNA was isolated and analyzed for a specific indel error, a result of non-homologous end joining (NHEJ) if double-strand break (DSB) was successfully created by the CRISPR/Cas9 complex. This could not be detected. The reason could be low efficiency of the CRISPR/Cas9 at the current targeting sites.

Despite not being able to confirm specific DSB created by CRISPR/Cas9, we still tried to perform a transfection of CRISPR together with the targeting vector. More than 200 colonies survived the antibiotic selection, about 50 clones have the insert integrated in their genome (screened with GFP primers - Supp Table 1), but none has correctly inserted the sequence at the site of BMP-2 gene (screened with validation primers flanked outside of homologous arms Supp Table 1).

From an experimental point of view, since specific DSB could not be confirmed, it is very unlikely that specific homology directed repair (HDR) can happen. Thus, it is crucial to optimize the sgRNA targeting site and to confirm the cutting activity by SURVEYOR assay. Recently, the preferred method to induce HDR is by utilizing the modified Cas 9 nickase (Cas9n), which cause DNA nick instead of DSB [29, 30]. In this method, two distinct sgRNAs in combination with Cas9n are working together to create two nicks, one on each stand of the target sequence, which is equivalent to inducing a DSB. However, the chance of unspecific binding of both sgRNAs in close proximity to each other causing a DSB is very low, making this nickase strategy a lot more reliable.

Another factor that greatly affects efficiency of CRISPR-assisted HDR is the size of the intended edit and the type of DNA templates that one could produce. For small insertion/edit of less than $100 \mathrm{bp}$, single-stranded DNA template (ssDNA) is often used to increase efficiency of HDR to up to $60 \%$, utilizing a pair of homologous arms as short as 50-80 bases [31]. For larger insertion including fluorescent reporter and selection cassette such as the one we were attempting, homologous arms of at least 800 bases is recommended [32]. Due to the difficulties to produce ssDNA longer than 200 bases, researchers have been using double-stranded DNA (dsDNA) cloned into bacterial plasmids. Quadro et al. developed a new technique, EasiCRISPR, to produce ssDNA template longer than 200 bases [33]. It was done by in vitro transcribing RNA encoding the template, and then using reverse transcriptase to create the complementary ssDNA. Tested in over a dozen loci, they have reported an increase in editing efficiency from $1-10 \%$ with dsDNA to $25-50 \%$ with ssDNA.

Overall, we have attempted to create a knock-in reporter system for BMP-2 gene in MG63 cell line. Although not successfully making the reporter cell line, the BMP-2 targeting template has been generated and verified by sequencing, paving the way to development of a BMP-2 reporter system in future. 


\section{References for Chapter 6 (BMP-2 reporter cell line)}

1. Urist, M.R. and B.S. Strates, Bone morphogenetic protein. J Dent Res, 1971. 50(6): p. 1392406.

2. Urist, M.R., Bone: formation by autoinduction. Science, 1965. 150(3698): p. 893-9.

3. Wozney, J.M., Bone morphogenetic proteins. Prog Growth Factor Res, 1989. 1(4): p. 26780 .

4. Even, J., M. Eskander, and J. Kang, Bone morphogenetic protein in spine surgery: current and future uses. J Am Acad Orthop Surg, 2012. 20(9): p. 547-52.

5. Yoon, B.S. and K.M. Lyons, Multiple functions of BMPs in chondrogenesis. J Cell Biochem, 2004. 93(1): p. 93-103.

6. Wozney, J.M., Overview of bone morphogenetic proteins. Spine (Phila Pa 1976), 2002. 27(16 Suppl 1): p. S2-8.

7. Lyons, K.M., R.W. Pelton, and B.L. Hogan, Organogenesis and pattern formation in the mouse: RNA distribution patterns suggest a role for bone morphogenetic protein-2A (BMP2A). Development, 1990. 109(4): p. 833-44.

8. Reddi, A.H. and A. Reddi, Bone morphogenetic proteins (BMPs): from morphogens to metabologens. Cytokine Growth Factor Rev, 2009. 20(5-6): p. 341-2.

9. Corradini, E., J.L. Babitt, and H.Y. Lin, The RGM/DRAGON family of BMP co-receptors. Cytokine Growth Factor Rev, 2009. 20(5-6): p. 389-98.

10. Schulz, T.J. and Y.H. Tseng, Emerging role of bone morphogenetic proteins in adipogenesis and energy metabolism. Cytokine Growth Factor Rev, 2009. 20(5-6): p. 523-31.

11. Zhang, H. and A. Bradley, Mice deficient for BMP2 are nonviable and have defects in amnion/chorion and cardiac development. Development, 1996. 122(10): p. 2977-86.

12. Ma, L. and J.F. Martin, Generation of a Bmp2 conditional null allele. Genesis, 2005. 42(3): p. 203-6.

13. Bandyopadhyay, A., et al., Genetic analysis of the roles of BMP2, BMP4, and BMP7 in limb patterning and skeletogenesis. PLoS Genet, 2006. 2(12): p. e216.

14. Tsuji, K., et al., BMP2 activity, although dispensable for bone formation, is required for the initiation of fracture healing. Nat Genet, 2006. 38(12): p. 1424-9.

15. Singh, A.P., et al., Influences of reduced expression of maternal bone morphogenetic protein 2 on mouse embryonic development. Sex Dev, 2008. 2(3): p. 134-41.

16. Wu, L.A., et al., Development and characterization of a mouse floxed Bmp2 osteoblast cell line that retains osteoblast genotype and phenotype. Cell Tissue Res, 2011. 343(3): p. 54558.

17. Barradas, A.M., et al., A calcium-induced signaling cascade leading to osteogenic differentiation of human bone marrow-derived mesenchymal stromal cells. Biomaterials, 2012. 33(11): p. 3205-15.

18. Zhang, J., et al., Cells responding to surface structure of calcium phosphate ceramics for bone regeneration. J Tissue Eng Regen Med, 2017. 11(11): p. 3273-3283.

19. Wu, J., et al., Generation and Characterization of a MYF5 Reporter Human iPS Cell Line Using CRISPR/Cas9 Mediated Homologous Recombination. Sci Rep, 2016. 6: p. 18759.

20. He, X., et al., Knock-in of large reporter genes in human cells via CRISPR/Cas9-induced homology-dependent and independent DNA repair. Nucleic Acids Res, 2016. 44(9): p. e85.

21. Kimura, Y., et al., Efficient generation of knock-in transgenic zebrafish carrying reporter/driver genes by CRISPR/Cas9-mediated genome engineering. Sci Rep, 2014. 4: p. 6545.

22. Pautke, C., et al., Characterization of osteosarcoma cell lines MG-63, Saos-2 and U-2 OS in comparison to human osteoblasts. Anticancer Res, 2004. 24(6): p. 3743-8.

23. Czekanska, E.M., et al., In search of an osteoblast cell model for in vitro research. Eur Cell Mater, 2012. 24: p. 1-17. 
24. Ryan, M.D., A.M. King, and G.P. Thomas, Cleavage of foot-and-mouth disease virus polyprotein is mediated by residues located within a 19 amino acid sequence. J Gen Virol, 1991. 72 ( Pt 11): p. 2727-32.

25. Skotzko, M., et al., Retroviral vector-mediated gene transfer of antisense cyclin G1 (CYCG1) inhibits proliferation of human osteogenic sarcoma cells. Cancer Res, 1995. 55(23): p. 5493-8.

26. Godiska, R., et al., Linear plasmid vector for cloning of repetitive or unstable sequences in Escherichia coli. Nucleic Acids Res, 2010. 38(6): p. e88.

27. Hockemeyer, D., et al., Genetic engineering of human pluripotent cells using TALE nucleases. Nat Biotechnol, 2011. 29(8): p. 731-4.

28. Dewey, R.A., et al., Chronic brain inflammation and persistent herpes simplex virus 1 thymidine kinase expression in survivors of syngeneic glioma treated by adenovirusmediated gene therapy: implications for clinical trials. Nat Med, 1999. 5(11): p. 1256-63.

29. Kocher, T., et al., Cut and Paste: Efficient Homology-Directed Repair of a Dominant Negative KRT14 Mutation via CRISPR/Cas9 Nickases. Mol Ther, 2017. 25(11): p. 25852598.

30. Ran, F.A., et al., Double nicking by RNA-guided CRISPR Cas 9 for enhanced genome editing specificity. Cell, 2013. 154(6): p. 1380-9.

31. Richardson, C.D., et al., Enhancing homology-directed genome editing by catalytically active and inactive CRISPR-Cas9 using asymmetric donor DNA. Nat Biotechnol, 2016. 34(3): p. 339-44.

32. Jasin, M. and R. Rothstein, Repair of strand breaks by homologous recombination. Cold Spring Harb Perspect Biol, 2013. 5(11): p. a012740.

33. Quadros, R.M., et al., Easi-CRISPR: a robust method for one-step generation of mice carrying conditional and insertion alleles using long ssDNA donors and CRISPR ribonucleoproteins. Genome Biol, 2017. 18(1): p. 92. 


\section{Supp Table 1. Primer sequences}

\begin{tabular}{|c|c|c|}
\hline Name & Purpose & Sequence \\
\hline $\begin{array}{l}\text { Left_arm } \\
\text { FWD }\end{array}$ & \multirow{2}{*}{$\begin{array}{l}\text { To clone the homologous left arm from } \\
\text { genomic DNA, with restriction site } \\
\text { (underline) and the } 2 \mathrm{~A} \text { sequence (bold) } \\
\text { added to the overhang }\end{array}$} & $\begin{array}{l}\text { 5'(CCAAAGAAGTAGATCCCTGTAAAT } \\
\text { G)3' }\end{array}$ \\
\hline $\begin{array}{l}\text { Left_arm } \\
\text { REV }\end{array}$ & & $\begin{array}{l}\text { 5'(AAAAAGCTAGCCTTGTCATCGTCG } \\
\text { TCCTTGTAATCGCGACACCCACAACC } \\
\text { CTCCAC)3' }\end{array}$ \\
\hline $\begin{array}{l}\text { Right_arm } \\
\text { FWD }\end{array}$ & \multirow{2}{*}{$\begin{array}{l}\text { To clone the homologous right arm } \\
\text { from genomic DNA, with restriction } \\
\text { site (underline) added to the overhang }\end{array}$} & $\begin{array}{l}\text { 5'(AAATGGCGCGCCTGTGGGTGTCGC } \\
\text { TAGTACAGC)3' }\end{array}$ \\
\hline $\begin{array}{l}\text { Right_arm } \\
\text { REV }\end{array}$ & & 5'(ATTTCCAACCCTTGATGAG) ${ }^{\prime}$ \\
\hline $\begin{array}{l}\text { Surveyor } \\
\text { FWD }\end{array}$ & \multirow{2}{*}{$\begin{array}{l}\text { To clone the CRISPR-Cas9 targeting } \\
\text { site for testing of cutting efficiency } \\
\text { (with Surveyor assay) }\end{array}$} & 5'(TGGTCACAGATAAGGCCATT)3' \\
\hline $\begin{array}{l}\text { Surveyor } \\
\text { REV }\end{array}$ & & 5'(CCATTATACTTCATGTGCTG)3' \\
\hline $\begin{array}{l}\text { Validation } \\
\text { FWD }\end{array}$ & \multirow{2}{*}{$\begin{array}{l}\text { To check for correct insertion of the } \\
\text { knock-in sequence (primers placed } \\
\text { outside homologous arm) }\end{array}$} & $\begin{array}{l}\text { 5'(GGTGAGAGAACAGACAACAGTTGG } \\
\text { GAAAGC)3' }\end{array}$ \\
\hline $\begin{array}{l}\text { Validation } \\
\text { REV }\end{array}$ & & 5'(TTCTGCTGGTAGTGGTCGGCGA)3' \\
\hline GFP_FWD & \multirow{2}{*}{$\begin{array}{l}\text { To check for the presence of GFP } \\
\text { reporter (regardless of correct } \\
\text { insertion) }\end{array}$} & 5'(ATGGTGAGCAAGGGCGAGGA)3' \\
\hline GFP_REV & & 5'(TTCTGCTGGTAGTGGTCGGCGA)3' \\
\hline
\end{tabular}




\section{Chapter 7 - Discussion}

B

one regeneration, a subject as old as the history of medicine itself, is likely to remain attractive to generations of scientists to come. It is because of the desire for perfection, a true regeneration, something that is deemed feasible or at least encouraged in many ways. Many animals, the salamander is a famous example, can readily regrow their limbs like a copy machine. We can only regenerate bone to some extent, not the whole bone and obviously not the whole limb.

Now facing this problem, which everyone is eager to solve, a surgeon, an engineer and a scientist each has different ideas about what approach to take. Surgeons are very practical people; their idea of bone regeneration is perhaps not too remotely different from that of Dr. Frankenstein. Practically speaking, organ transplantation works. Perhaps, it can be perfected in a way that makes regeneration research a redundant thing?

Vascularized bone graft is a perfect example of how the surgeons might regenerate bone in large and challenging defects [1]. The intact vascularization of the graft helps to preserve osteocytes, which otherwise would become necrotic and trigger bone resorption, thus accelerating the healing process. Not only bone but transplantation of whole hand or upper limb, consisting of skin, muscle, nerves, blood vessels and bone, has recently been performed successfully, thanks to advances in surgical techniques and immunosuppressive therapy [2]. The field of vascularized composite allotransplantation (VCA) is growing and provides immediate benefit for the patients, including those who lost their faces and upper extremities [3]. However, this type of surgery is very technically demanding to the surgeons, not to mention the already extreme donor shortage. Put together, the prospect of having routine limb transplantations in the future seems too much trouble. And technically speaking, it's not regeneration.

The engineers will of course have their solutions, too. Their approaches are, for example, to restore the mechanical function of bones with scaffolds, fixation devices, and prosthetics. From an engineering point of view, restoring the mechanical function of bone only is just too easy, and perhaps a bit boring. In fact, engineers have developed many devices - pins, rods, screws, plates, scaffolds of all size, shape, strength, porosity and materials - that orthopaedic surgeons rely on to fix bone fractures. Engineers and surgeons have long been a good team when it comes to surgical tool development.

However, what really catches attention of the engineers is something more sophisticated, something like a robotic limb that is indistinguishable from its human counterpart. One example is the Modular Prosthetic Limb (MPL), the current most advanced robotic arm with mindcontrol for patients with below-elbow amputations [4]. This robotic arm is totally controlled by the patient's thought, to the level of individual digits, with haptic feedback to provide sensation of the grasping objects. In this era of advance engineering and manufacturing, the evolution of 
such prostheses is incredibly fast. With little doubt, the engineers will be the first to have the solid answer for the 'regeneration' of limbs for the amputees.

Now that is obviously not the kind of 'regeneration' that biologists know, but functionally speaking, it could be one feasible approach toward immortality. "The spirit indeed is willing but the flesh is weak" - said Jesus to his disciples. Could it be that what is really needed from a human being is just a spirit after all? The idea of transferring the human's consciousness into a robot body was thought of long ago, science-fiction of course, such as in the novella Izzard and the Membrane by Walter M. Miller [5]. Until now, the transferring part is still fictitious, but the robot body part is getting more realistic than ever.

While the surgeons and the engineers have their preferred approaches to follow, the scientists are facing an overwhelming number of possible directions. The questions related to the process of regeneration are too huge and diverse. These are questions about the cells, the genes, the growth factors, the mechanisms, the interactions, what controls what, what limits their capabilities, and how to unlock their potential? And to each of these questions lies a different approach to find the right answer.

Some scientists are looking to the salamanders and other similar organisms to crack a universal code for regeneration. They soon realized that the immune system play an important role in the process $[6,7]$. Later, Godwin et al. demonstrated that macrophages are essential for limb regeneration of salamanders [8]. Systemic macrophage depletion by an immune suppressive drug within $24 \mathrm{~h}$ of limb amputation resulted in wound closure and failure of limb regeneration. However this process could be restored by re-amputation once the endogenous macrophages recovered. At the same time, other scientists using a genetic approach to delete macrophage lineage also came to a similar conclusion that resident macrophages are indispensable to bone healing and regeneration [9, 10]. Since then, the immune cells have gained significant research interest among bone scientists [11]. Something that was initially thought of as remotely related to bone can become an approach to bone regeneration.

Of course the macrophage is just one player in the bone regenerating process. Talking about the cells, bone scientists are currently on a quest to identify the 'bone stem cell'. Bone can only be formed by osteoblasts - the bone forming cells. And a cell must derive from another cell. Thus, a source of adult stem cells must exist in the body to take care of bone repair through life. The questions are: what exactly is the origin of these cells; where do they reside; how do they naturally contribute to the bone repair; are they stem cells?

Scientists once thought they have identified these stem cells - which they named the mesenchymal stem cells (MSCs) - and methods were developed to isolate, expand and differentiate them to bone/cartilage/adipose cells in vitro. MSCs are isolated from bone, in the periosteum, endosteum, osseous tissue or bone marrow, and in the tissues that they can differentiate into, such as fat tissue. Indeed, transplantation of MSCs in vivo can result in formation of ossciles, consisting of bone, cartilage and bone marrow [12, 13]. However, to satisfy the "stem cell" definition, the cells must demonstrate self-renewal capacity in vivo through serial transplantations. For MSCs, this level of evidence has not been achieved, suggesting that MSCs might not be stem cells after all [14, 15]. Without unlimited self-renewal 
capacity, the cells are short-lived and can hardly be useful for long term regeneration purpose. The search for the bone stem cells continues.

Meanwhile, some other scientists are busy investigating the components of the bone extracellular matrix. They have a different approach: if the stem cells are already available in the body, what is really needed is a set of instructions. One example that proves this approach is the growth factor bone morphogenetic protein-2 (BMP-2). Discovered in the 1960s, it took 40 years for the products to be approved by the US Food and Drug Administration (FDA). The mechanism of BMP-2 has been extensively studied, numerous lab animal models tested, and hundreds of clinical trials completed [16]. BMP-2 has been successful in treating complicated bone problems such as persistent fracture non-unions, distraction osteogenesis, and spine pseudarthrosis $[17,18]$. Scientists know they can make bone anywhere in the body with BMP-2 [19]. The problem is they cannot really control the process, which is now similar to driving a car using only the accelerator. Engineers, of course, are eager to lend a helping hand. A plethora of delivery systems have been created to control the timing and dosages of the drug [20]. One thing is certain: no limb has yet to be regenerated using BMP-2 therapy alone.

Scientists can never end a discussion without mentioning about genetics. Indeed, the genes make all the difference. But scientists realized that humans also possesses very similar genes to the salamander, the ones that orchestrate embryonic limb growth and limb regeneration [21]. For reasons that scientists are dying to know, these human genes are not activated during injuries and thus we do not regrow our limbs. For those who truly believe in the answer around the corner, the genetic approach is the way to go.

Biogerontologist Marios Kyriazis has an idea - a theory of extreme lifespans through perpetual-equalizing intervention - that opposes natural selection. This theory suggested that at some point in time, the human being will continue to evolve his intelligence by living indefinitely, not the other way round, i.e. by natural selection [22]. It is an interesting theory to think about, particularly when we are stepping into an era when clinical gene editing is a reality, a proof of concept that evolution is at our hands. The human beings always live in the future; we can foresee events many years in advance. There are countless examples of things that were considered mere fiction just decades ago - the internet, the smartphone, space travel, and artificial intelligent to name a few. With some luck, the human being will eventually re-design his body and add human immortality to the list of mission accomplished.

In the quest to find a master switch for regeneration, everyone plays a different role; everyone has his/her preferred approaches. Some people would like to study a specific organ/tissue - the heart, the brain, the skin, the bone etc. Some would like to look deep into the biological mechanisms of regeneration. With all these ongoing efforts, and particularly with the increasing help of technology - artificial intelligence, that it - there is no reason to not believe in true human regeneration. 


\section{References for Discussion}

1. Rizzo, M. and S.L. Moran, Vascularized bone grafts and their applications in the treatment of carpal pathology. Semin Plast Surg, 2008. 22(3): p. 213-27.

2. Elliott, R.M., S.M. Tintle, and L.S. Levin, Upper extremity transplantation: current concepts and challenges in an emerging field. Curr Rev Musculoskelet Med, 2014. 7(1): p. 83-8.

3. Bueno, E.M., et al., Vascularized composite allotransplantation and tissue engineering. J Craniofac Surg, 2013. 24(1): p. 256-63.

4. Perry, B.N., et al., Initial Clinical Evaluation of the Modular Prosthetic Limb. Front Neurol, 2018. 9: p. 153.

5. Roberson, W.H., Walter M. Miller, Jr.: A Reference Guide to His Fiction and His Life2011: McFarland, Incorporated, Publishers.

6. Schotte, O.E. and R.E. Sicard, Cyclophosphamide-induced leukopenia and suppression of limb regeneration in the adult newt, notophthalmus viridescens. J Exp Zool, 1982. 222(2): p. 199-202.

7. Fahmy, G.H. and R.E. Sicard, A role for effectors of cellular immunity in epimorphic regeneration of amphibian limbs. In Vivo, 2002. 16(3): p. 179-84.

8. Godwin, J.W., A.R. Pinto, and N.A. Rosenthal, Macrophages are required for adult salamander limb regeneration. Proc Natl Acad Sci U S A, 2013. 110(23): p. 9415-20.

9. Wu, A.C., et al., Unraveling macrophage contributions to bone repair. Bonekey Rep, 2013. 2: p. 373.

10. Alexander, K.A., et al., Osteal macrophages promote in vivo intramembranous bone healing in a mouse tibial injury model. J Bone Miner Res, 2011. 26(7): p. 1517-32.

11. Sinder, B.P., A.R. Pettit, and L.K. McCauley, Macrophages: Their Emerging Roles in Bone. J Bone Miner Res, 2015. 30(12): p. 2140-9.

12. Sacchetti, B., et al., Self-renewing osteoprogenitors in bone marrow sinusoids can organize a hematopoietic microenvironment. Cell, 2007. 131(2): p. 324-36.

13. Mendez-Ferrer, S., et al., Mesenchymal and haematopoietic stem cells form a unique bone marrow niche. Nature, 2010. 466(7308): p. 829-34.

14. Caplan, A.I., Mesenchymal Stem Cells: Time to Change the Name! Stem Cells Transl Med, 2017. 6(6): p. 1445-1451.

15. Robey, P., "Mesenchymal stem cells": fact or fiction, and implications in their therapeutic use. F1000Res, 2017. 6.

16. Gautschi, O.P., S.P. Frey, and R. Zellweger, Bone morphogenetic proteins in clinical applications. ANZ J Surg, 2007. 77(8): p. 626-31.

17. Lissenberg-Thunnissen, S.N., et al., Use and efficacy of bone morphogenetic proteins in fracture healing. Int Orthop, 2011. 35(9): p. 1271-80.

18. Bodalia, P.N., et al., Effectiveness and safety of recombinant human bone morphogenetic protein-2 for adults with lumbar spine pseudarthrosis following spinal fusion surgery: A systematic review. Bone Joint Res, 2016. 5(4): p. 145-52.

19. Scott, M.A., et al., Brief review of models of ectopic bone formation. Stem Cells Dev, 2012. 21(5): p. 655-67. 
20. Agrawal, V. and M. Sinha, A review on carrier systems for bone morphogenetic protein-2. J Biomed Mater Res B Appl Biomater, 2017. 105(4): p. 904-925.

21. Alibardi, L., Review: Limb regeneration in humans: Dream or reality? Ann Anat, 2018. 217: p. 1-6.

22. Kyriazis, M., Practical applications of chaos theory to the modulation of human ageing: nature prefers chaos to regularity. Biogerontology, 2003. 4(2): p. 75-90. 


\section{Valorization addendum}

$\mathrm{T}$

he knowledge generated in this thesis has great potential to be utilized for both social and economic purposes. Any disease or health problem has a dramatic impact on the well-being of people, and bone diseases or conditions are no exception. Problems such as lower back pain and non-union are one of the leading causes of years lived with disability in many countries worldwide. One of the goals of this thesis was to generate knowledge that could eventually be applied to treat these patients and thereby improve their life quality.

As we are approaching the 2020 s, the science of bone regeneration is facing a big challenge: the lack of novelty in the approaches taken and therapies available. Clinicians are stuck with a century-old method, the autologous bone graft. Or they may elect take a risk by using bone morphogenetic protein-2 (BMP-2) or unproven methods (e.g., mesenchymal stem cell therapies). And certainly many will be confused by what appears to be a wide selection of bone graft substitutes that are essentially the same (namely a combination of either allogenic bone, type I collagen and/or calcium phosphate). There is a great need for some breakthroughs in the science and clinical application of bone regeneration.

Throughout this thesis, we have explored several different approaches to look for a solution for the regeneration of bone. It is noteworthy that we used the cell line ATDC5 three times (in chapters 2, 3 and 4). There is a good reason for this. ATDC5 is a mouse cell line with the intrinsic potential to become hypertrophic chondrocytes. The collagen matrix of hypertrophic chondrocytes is rich in type $\mathrm{X}$ collagen, which is a natural template for bone formation in the body during growth and fracture healing.

Type $\mathrm{X}$ collagen is very rare in nature, and currently only available for research from sources such as placenta tissue, at very high price. It is why type $\mathrm{X}$ collagen, despite its relevance to bone formation, has not been used for bone regeneration. There is no clinical product on the market using type X collagen at the moment. Instead, type I collagen is used, isolated from sources such as bovine skin, which may not the best type of material for bone formation. Thus, there is a big opportunity for a product comprising type $\mathrm{X}$ collagen for bone regeneration.

In chapter 2, we did a high throughput screen to search for a drug that can stimulate the cell ATDC5 to produce more collagen matrix. We found and successfully validated a compound called tetradecylthioacetic acid (TTA). There are two possibilities to use the knowledge from this chapter. First, using TTA, we can readily optimize a culture protocol to stimulate ATDC5 cells to increase their collagen production, in particular type X collagen, which is discussed further below. Second, the method of using a collagen-binding fluorescent probe for high throughput screening can be adapted to screen on other cell types. For example, using it with skin progenitor cells could find a molecule that promotes the formation of a collagen matrix suitable for skin regeneration. 
In chapter 3, we put our effort into upscaling the culture of ATDC5 cells in vitro. The most common method to culture these cells is using the surface of the plastic vessels (such as 300 $\mathrm{cm}^{2}$ flasks), which is a 2D culture system. While these are convenient, ATDC5 thrive best in a $3 \mathrm{D}$ environment, where the cells are always in contact to each other. The work in chapter 3 described an upscaling culture method in which ATDC5 cells are grown as small aggregates of 1000 cells, formed in a mold containing thousands of micro-wells. We called these aggregates: micro-tissue-engineering cartilage (MiTEC). We demonstrated that MiTEC grew much better and produced collagen matrix in high quantity and quality. This method of micro-aggregate cell culturing is suitable to upscale to a much bigger scale using bioreactor vessels, and potentially producing large quantities of hypertrophic matrix biomass rich in type $\mathrm{X}$ collagen.

Next, in chapter 4 , we did some basic research to prove the concept that surface topography of a material can be as potent as growth factors for directing cell fate. All materials implanted in the body are in contact with cells, and how cells respond to the materials influences the survival of the implants. In bone regeneration, surgeons have long realized that materials with rough surfaces are superior to those with smooth finishes for bone integration. But the mechanism is far more complicated than that, and we are still learning every day.

In order for this knowledge from chapter 4 to be applicable to life, more research needs to be done. However, as evidence builds for the mechanisms and reproducibility, the science of surface topography can be widely applied in almost all aspects of medicine. One can foresee its use in replacing or augmenting growth factor therapy and it can be used in vitro (e.g., in manufacturing of type X collagen matrix) or in the clinic (e.g., to modify surface topography of dental implants).

In chapter 5, we attempted to devise a reporter cell line for the gene BMP-2. There has not been a cell line that can faithfully produce a fluorescent signal concomitant to the change of BMP-2 expression. Such a reporter cell line has tremendous use for scientists, who need to understand the role of BMP-2, a universal body growth factor, in organogenesis and the natural healing response. A BMP-2 reporter cell line could also be used for essentially any kind of high throughput screening such as drug screening or material screening, because of its convenient reporting properties. However, generating such a cell line is a difficult task, and we have complete perhaps $50 \%$ of the job. What is left to be done is the optimization of the genome editing tools, such as CRISPR, to efficiently induce a double-strand break at the desired location.

Now to elaborate on the potential of chapters 2-4 and the use of ATDC5 cells to produce collagenous matrix rich in type $\mathrm{X}$ collagen (hypertrophic cartilage matrix).

Type $\mathrm{X}$ collagen has a potential to revolutionize bone regeneration, but just getting enough type $\mathrm{X}$ collagen for a single experiment is already a challenge. Take one clinical example, treating severe degenerative intervertebral disc diseases with spinal fusion. The aim of this procedure is to stimulate bone fusion between two or more vertebrae in the affected region. Currently, recombinant human BMP-2 delivered in a type I collagen sponge, is recommended to use for this procedure by the US FDA. To guarantee a successful outcome, a very large dose of BMP-2 is often used by surgeons, which results in not only adverse effects but also an 
expensive therapy. The fact is that surgeons depend solely on BMP-2 (or BMP-7) to regenerate bone, while the type I collagen sponge is simply a carrier.

But type I collagen alone is often used as a negative control in animal models of bone regeneration. Why is it that a material with no intrinsic bone forming capacity is used so often? Only because of its availability. Bovine or porcine type I collagen can be easily harvested from skin. If type X collagen could be available in only one tenth of the quantity of type I collagen, it would have a very good chance of competing in the market.

We all know that Maastricht produced the first million-dollar burger, made from bovine muscle cultured in the lab, and several companies followed to bring the cost down. If cells can be cultured for food at this high cost, there is absolute no reason why cells cannot be cultured for medical needs at similar cost. Type $\mathrm{X}$ collagen matrix has a great potential for bone regeneration, which has been shown in a rich body of literature, but steps need to be taken to bring a product into a clinical trial.

The work in this thesis has consistently suggested the use of the ATDC5 cells in producing such a collagen matrix. We have shown the feasibility to grow MiTEC in the lab, and have shown the ability to produce $1 \mathrm{CC}$ of decellularized matrix from four 12-well plates. The next step is to produce $100 \mathrm{CC}$ of matrix, using similar plates. For reference, a single interbody spinal fusion case needs $2-10 \mathrm{CC}$ of collagen matrix. This amount would be sufficient to bring into an animal trial.

Next, engineers need to be involved to up-scale the culture of MiTEC in larger vessels. MiTEC can be cultured in suspension, thus eliminating the need for surfaces. Engineers should be able to make bioreactors in the size of $10,50,100$ and up to $1000 \mathrm{~L}$, with sensors to control temperature, $\mathrm{pH}$, and chemical levels. Every batch of ATDC5 culture can take up to 14 days to produce.

ATDC5 matrix can also be used directly as a decellularized matrix, as demonstrated in chapter 3, or can be used as a raw material for the isolation type $\mathrm{X}$ collagen. The benefit of further purifying the matrix is to ensure a medical grade product can be obtained through stringent manufacturing processes. Medical grade type X collagen can be applied in all bone site injuries (where type I collagen is currently used), from fracture healing, spinal fusion to filling of dental socket. Without a doubt, a type X collagen sponge will offer another interesting approach for future bone regeneration approaches.

Overall, this thesis has tapped into several approaches that can be useful for the research and development of products for bone healing and regeneration. Of which, the knowledge from chapter 2, 3 and 4 can be most applicable for the development of a type X collagen matrix from cultured ATDC5 cells. 


\section{Summary}

\section{Bone regeneration: Exploring a plethora of approaches}

Bone related disorders affect millions of people worldwide and are the leading cause for years lived with disabilities in many countries. Research in bone healing and regeneration is one of the earliest examples of medical research, with a direct impact on increasing the quality of life. Scientific and technological advances of the $21^{\text {st }}$ century, particularly in the areas of genomics and proteomics, have generated a huge amount of data, but an understanding the mechanisms, pathway, and cell types involved is far from complete.

Chapter 1 of this thesis provides an up-to-date literature review about the components of bone and their regenerative capacity. Bone consists of macro-level tissues such as periosteum, osseous tissue, endosteum and bone marrow, which are useful for their utilization by the orthopaedic surgeons. But laboratory scientists prefer to work on a different scale, namely the microscopic composition of bone, including the bone cells and extracellular matrix proteins. Each of these components has been tested for their ability to regenerate bone in vitro as well as in vivo, which is the focus of this literature review.

In chapter 2, we performed a high throughput screen to search for a drug that stimulates collagen production of ATDC5 cells. Bone formation starts with the production of collagen matrix by osteoblasts. Increasing collagen production could have a benefit for bone formation. After the screen, we found and validated a drug called tetradecylthioacetic acid (TTA). TTA consistently induces collagen production from ATDC5 cells at bone gene and protein level. However, we did not see the same effect of TTA on human chondrocytes and human mesenchymal stem cells. Thus TTA can be used to improve the in vitro culture of ATDC5.

For chapter 3, we used a 3D culture system to grow ATDC5 cells for the purpose of upscaling its collagen matrix production. The cells were cultured in small aggregates of 1000 cells, which we called micro-tissue-engineering cartilage (MiTEC). This allows the cells to be in contact with each other, while facilitating nutrient and waste exchange to the culture medium. MiTEC can grow significantly in size, and prodice the desired extracellular matrix quantity and quality. We demonstrated that this 3D culture system is suitable to grow ATDC5 in the lab for manipulating experiments, while has great potential for upscale manufacturing.

In chapter 4, we experimented with a novel approach of using topographical cues to influence cell fate. Topographical patterns that could induce early osteogenic differentiation of mesenchymal stem cells (from a previous screen), were tested to evaluate their effect on ATDC5 cells. The results showed that some patterns also stimulate hypertrophic differentiation of ATDC5, proving the concept of topography inducing cell fate. These patterns can be used to improve the in vitro culture of ATDC5 cell line.

Finally, in chapter 5, we attempted to devise a reporter cell line for the growth factor bone morphogenetic protein-2 (BMP-2) utilizing the CRISPR-Cas9 gene editing tool. We successfully produced the DNA template for the knock-in strategy, consisting of the reporter 
gene GFP, the puromycin resistant cassette and 2 homologous arms for BMP-2 locus. The template was validated by sequencing. However, the single guide RNA targeting of the BMP-2 locus was not successful, and thus none of the transfected cells carries the correct insert. More work needs to be done to finish the generation of this BMP-2 reporter cell line.

Overall, this thesis has explored several different approaches to study bone regeneration: a pharmaceutical high throughput drug screening approach, an upscaling 3D-cell culture approach, a novel topographical approach and a conventional genetic approach. Of the plethora of approaches that one could take, the author has chosen these broadly different ones in order to get exposed to as many laboratory techniques as possible. Besides, the author has an ambition for commercialization, thus every chosen approach has its own valorisation potential. To this end, the author still cannot answer the question: what is the best approach for bone regeneration? Perhaps, a lot more effort is needed to complete this mission. One thing is clear; the scientists are all on it. 


\section{Samenvatting}

\section{Botregeneratie: een verkenning van meerdere benaderingen}

Bot gerelateerde aandoeningen komen wereldwijd voor bij miljoenen mensen en zijn in veel landen de belangrijkste oorzaak voor het leven met een handicap. Onderzoek naar botgenezing en -regeneratie is een van de vroegste voorbeelden van medisch onderzoek met een directe invloed op het verhogen van de kwaliteit van leven. Wetenschappelijke en technologische ontwikkelingen in de 21e eeuw, met name op het gebied van genomics en proteomics, hebben een enorme hoeveelheid aan data gegenereerd. Echter, ons inzicht in de mechanismen, pathways en celtypes die hierbij betrokken zijn, is verre van compleet.

Hoofdstuk 1 van dit proefschrift geeft een actueel literatuuroverzicht van de botcomponenten en hun regeneratieve vermogen. Bot bestaat uit weefsels op macroniveau zoals periosteum, botweefsel, endosteum en beenmerg, die nuttig zijn voor gebruik door orthopedisch chirurgen. Maar wetenschappers werken het liefst op een andere schaal, namelijk de microscopische samenstelling van het bot, inclusief de botcellen en extracellulaire matrixeiwitten. Al deze componenten zijn getest op hun vermogen om bot te regenereren, zowel in vitro als in vivo, wat de belangrijkste focus is van het literatuuroverzicht in dit hoofdstuk.

In hoofdstuk 2 hebben we een high throughput screen uitgevoerd om een medicijn te vinden dat de productie van ATDC5-collageen stimuleert. Botvorming begint met de productie van collageenmatrix door osteoblasten. Het verhogen van de productie van collageen zou voordeelig kunnen zijn voor de botvorming. Na de screen vonden en valideerden we een medicijn genaamd tetradecylthioacetic acid (TTA). TTA induceert consequent collageenproductie door ATDC5-cellen op gen- en eiwitniveau. We zagen echter niet hetzelfde effect van TTA op menselijke chondrocyten en menselijke mesenchymale stamcellen. Daarop concludeerden we dat TTA gebruikt kan worden om de in vitro kweek van ATDC5 cellen te verbeteren.

Voor hoofdstuk 3 hebben we een 3D-kweeksysteem gebruikt om ATDC5 cellen te laten groeien met als doel om de productie van collageenmatrix op te hogen. De cellen werden gekweekt in kleine aggregaten van 1000 cellen, die we micro-tissue-engineering cartilage (MiTEC) noemden. Hierdoor kunnen de cellen met elkaar in contact komen, terwijl de uitwisseling van voedingsstoffen en afvalstoffen naar het kweekmedium wordt vergemakkelijkt. MiTEC kan aanzienlijk in omvang groeien en daarmee de gewenste hoeveelheid en kwaliteit aan extracellulaire matrix produceren. We hebben aangetoond dat dit 3D-kweeksysteem geschikt is om ATDC5 cellen in het laboratorium te laten groeien voor het beïnvloeden van experimenten, terwijl het daarnaast de mogelijkheid biedt voor het opschalen van de productie.

In hoofdstuk 4 hebben we geëxperimenteerd met een nieuwe benadering voor het gebruik van topografische modificaties om het gedrag van cellen te beïnvloeden. Topografische patronen die in een vorige screen vroege osteogene differentiatie van mesenchymale stamcellen induceerden, werden getest om hun effect op ATDC5 cellen te evalueren. Uit de resultaten bleek dat sommige patronen ook hypertrofische differentiatie van ATDC5 stimuleren, wat 
bewijst dat topografie inderdaad het gedrag van de cel beïnvloedt. Deze topografische patronen kunnen worden gebruikt om de in vitro kweek van ATDC5 cellijn te verbeteren.

Tot slot, in hoofdstuk 5, hebben we getracht een reportercellijn te ontwerpen voor de groeifactor bone morphogenetic protein-2 (BMP-2) met behulp van het CRISPR-Cas9 genediting systeem. We hebben met succes de DNA-template voor de knock-in-strategie geproduceerd, bestaande uit het reporter gen GFP, de puromycine-resistente cassette en twee homologe armen voor de BMP-2-locus. De template werd gevalideerd door middel van sequencing. Echter, de single-guide RNA-targeting van de BMP-2 locus was niet succesvol waardoor geen van de getransfecteerde cellen de juiste insertie bevatten. Er moet daarom meer werk worden verricht om deze BMP-2-reportercellijn te voltooien.

Over het algemeen heeft dit proefschrift verschillende benaderingen onderzocht om botregeneratie te bestuderen: een farmaceutische high-throughput aanpak van drugsscreening, een opschaling van de 3D-celcultuur, een nieuwe topografische aanpak en een conventionele genetische aanpak. Van de vele verschillende benaderingen die mogelijk zijn, heeft de auteur bovenstaande keuzes gemaakt om met zoveel mogelijk laboratoriumtechnieken ervaring op te kunnen doen. Bovendien heeft de auteur een ambitie voor commercialisering, dus heeft elke gekozen aanpak zijn eigen valorisatiepotentieel. De auteur kan echter nog geen antwoord geven op de vraag welke benadering het beste is voor botregeneratie. Misschien is er veel meer inspanning nodig om deze missie te voltooien. Eén ding is wel duidelijk: wetenschappers zitten er bovenop. 


\section{Acknowledgment And a little bit about the journey}

I

t took me a pretty long time ( 8 years, or about $10 \%$ of a good lifetime!) to reach this end the $\mathrm{PhD}$ process, an amazing journey which offered me a lot more than what I coud've asked for. THANK YOU, who happened to accompany me, briefly or all the way, in this journey. With a hope to not forget anyone that was important to me during this time, I thought I'd tell a short story.

It all started with an email, "Come over to our lab for a visit and interview, we'll pay your travel expenses and accommodation". I doubt whether you're still doing this, Jan, but nobody in their right mind would deny such an amazing offer. So I took the flight to Amsterdam, hopped on the train to Enschede, arrived at the Tissue Regeneration (TR) department and gave a 30minute presentation. Clemens was there, upon my note that I enjoyed cleaning test-tubes after classes at high school, he said: "you're hired". It was really straight forward (sort of)!

Work started one month later. My first-ever office had a nice view of the olive garden, shared between four students. Björn was a cool guy with sophisticated ideas. Jeroen would be able to spell out a name for any paper you mention, he's our live Pubmed. Charlene has a very entrepreneurship mind.

My mentor, Ana (Baradas), guided me through almost every lab protocols. She even told me what to eat, where to go in Enschede, great mentor! Then I met Hugo, who taught me to do qPCR the fun (and right) way and inspired me with high throughput screening. Little did I know what Ana and Hugo were to get married soon, I was super lucky to witness their heavenly arranged ceremony in Vatican chapel (no kidding!).

The Portuguese team, David and Joana, Ana (Rodrigues), João, and Carlos was always a good place to have a nice dinner with. Plus, David and I have an obsession for incredible ideas (still waiting for your homemade shampoo brand), and we have been to many places together (Castelo Branco, Phan Rang, Singapore), attending each other's wedding, unforgettable time.

The Chinese team, Jingwei, Honglin, Lei Lei, Bin and Ling was another good place to have a chat and some tasty snacks too. Praise the dumplings handmade by Jingwei; they're juicy even when they're cold. Bin was a tough guy; Western blot and qPCR, nobody can beat him. Ling, hello USA! Honglin and Lei Lei brought the Chinese mafia to another level!

The Jan-de-Boer's team members: Joyce, Eelco, Nick, Frits, Nathalie, Karolina, Max, Alex, Hemant, Anindita, Steven, Aurélie and Dennie: you guys rocked! Joyce, you inspired me to get my Dutch driving license. Eelco, great time on the A1 highway with you, every other week to Utrecht for mouse experiments, more time on the road than in the lab, unbelievable! Nick, I'm glad you liked the PCR song that I played in that lesson. Frits, thanks for making all those Topo-chips! Nathalie, your RNA kit supply never went short, great job! Karolina, I'll never forget our one-day trip across Holland to rescue your rats. Alex, thank you for the brilliant Rwork that you've done for my data, I will never be able to comprehend it myself. Max, I can 
listen to you all day! Hemant, you're a smart chap! Anindita, you're not only an amazing colleague but also such a good friend, thank you for everything! Steven and Aurélie: I can't thank you enough for helping with the TopoChip manuscript. Dennie, I owe you a very good case of beers, thank you for all the preparation that you have done for my defense.

Anouk, Jacqueline, Marlose and Tom provided the best technical support you'd ever need in the lab, their protocols never failed! Audrey was the person to go for when you're in any kind of needs, from printer ink to conference tickets, really appreciate your help. The bosses, Clemens, Jan, Pamela, Lorenzo, and Roman were always there not only to scare you but also to give abundant supports. I had two 15-min sections with Clemens to talk about some vague random ideas, which took 2 months to book appointment for; still I thought it's very cool. I had many hours of training with my supervisor - Jan - but the section that impressed me the most was when he trained me to open a beer bottle using another beer bottle (de Biesbosch, 2011), incredible! At that moment I decided that I needed to learn more from this man. Of course I will never forget your other lessons either - all the genetic stuffs - very useful indeed. Pamela sometimes seemed to be a bit strict with her razor-sharp look, but she otherwise gave very good advices. Technical discussion with Roman was always problem solving. And Lorenzo is a very good listener too.

At day, I did cell culture; when the night felt, I followed Hemant to his all-time-favorite BD pathway microscope. He used to live there literally. Sometimes a working night in the lab was even livelier than a working day. Gulistan, my first ever student, who obtained her $\mathrm{PhD}$ several months earlier than me, would do some serious experiments. Anne would definitely sit by the SEM, exploring the surface of Mars (otherwise known as scaffolds). Andrea and his Italian fellows would patrol around, discussing about electrospinning, great Tiramisu by the way! Erik, then still experimenting with his embryoid bodies, showed me the brilliant micro-well system, which I used a lot later. Anindita would run the histolab all night, with her daughter playing in the fish ball (the meeting room). Niloofar, Feb, Parthiban and Zyrian would run the molecular lab, laughing all the time. It was pretty incredible atmosphere; I wonder what made it so good those days.

My journey was a like marathon, the last quarter of it was tough. I would not have made it without your help, Vanessa. You were the paramedic running alongside and fed me a cookie every now and then, cheering me to the finish line. I never knew that you've done all the correction for my thesis during your last trimester. Big kudos to you, co-promotor!

Simon and Vic, you have been more than supporting me, you have given me the chance to continue to grow as a young scientist. I take it as a mission to help you with your sweetbusiness.

Of course, life is not only about work, so I was taught. If it was all about work, I would not have met my girlfriend, who is now my wife, back then since the first year in Holland. Tramie, you are such a loving and patient wife, the only one who doesn't complain too much about me working on this thesis for such a long time. Unofficially, you even helped me to do some lab work at night with the hope to accelerate the process. The little one, Sofia, when you're grown up you will read this, you're my little supporter too - it's called motivational support, honey. I 
hope you'll find a good motivation in your life too. Last but not least, my family has always been available when I needed them, spiritually and financially, thank you Dads, Mums, sisters, brothers, and everyone.

\section{Le Quang Bach}

05-Nov-18 


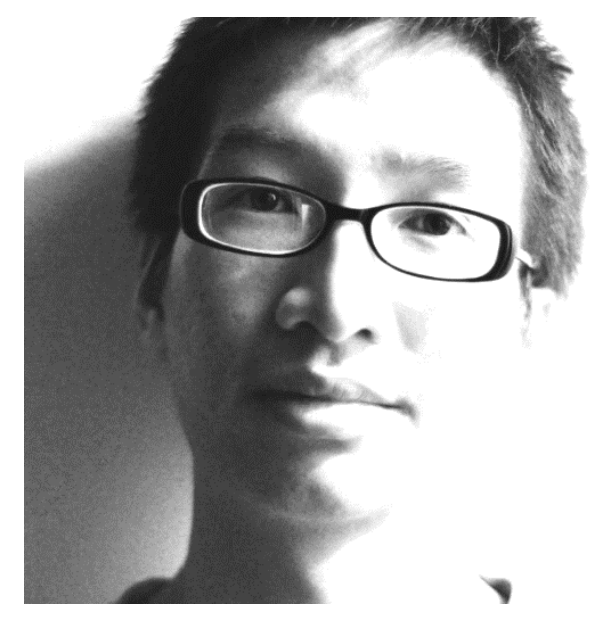

\section{About the author}

Le Quang Bach was born on November $15^{\text {th }} 1986$ in Hanoi, Vietnam. He did his high school from 2001-2004 at Hanoi-Amsterdam High School, majoring in chemistry. In 2004, he took part in the yearly university entrance exam in Vietnam and secured a place at Hanoi Medical University, being one of the two students nationally with perfect scores (30/30). After 2 years, he decided to pursue sciences instead, taking a scholarship to study a BSc Biomedical Science (2006-2009) at the University of Sunderland, UK. Having graduated with a First Class Honours, he was also awarded the Presidents Prize from the Institute of Biomedical Sciences, UK, for academic distinction. From 2010-2014, he performed his doctorate training at the Department of Tissue Regeneration chaired by Prof. Clemens van Blitterswijk (University of Twente), under the supervision of Dr. Jan de Boer. He then continued his bone regeneration research in the lab of Prof. Simon Cool and Prof. Victor Nurcombe at the Agency for Science, Technology and Research in Singapore. 